\title{
Comparison of Thiyl, Alkoxyl, and Alkyl Radical Addition to Double Bonds: The Unusual Contrasting Behavior of Sulfur and Oxygen Radical Chemistry
}

Isa Degirmenci*a,b and Michelle L. Coote*b

${ }^{a}$ Ondokuz Mayls University, Chemical Engineering Department, Samsun, 55139, Turkey.

${ }^{b}$ ARC Centre of Excellence for Electromaterials Science, Research School of Chemistry, The Australian National University, Canberra, ACT 2601, Australia.

\section{Supporting Information}

Table S1. Calculated charge transfer energies $\left(R^{-} A^{+}\right.$and $\left.R^{+} A^{-}, e V\right)$ and forming bond lengths in the transition states $(d, \AA)$ a

Table S2. HOMO and LUMO Orbital Energies of the Reactants ${ }^{\mathrm{a}}$................................................... 3

Table S3. Total Energies at Various Levels of Theory, Thermal Correction, Zero Point Vibrational Energies, High Level Corrections, Entropies and Enthalpies (units are Hartrees unless otherwise noted)

Table S4. MP2/6-31G(d) Optimized Geometries 
Table S1. Calculated charge transfer energies $\left(R^{-} A^{+}\right.$and $\left.R^{+} A^{-}, e V\right)$ and forming bond lengths in the transition states $(\mathrm{d}, \AA)^{\mathrm{a}}$

\begin{tabular}{|c|c|c|c|c|c|}
\hline & Radical & Substrate $^{b}$ & $\mathrm{~d}(\AA)$ & $\mathrm{R}^{-} \mathrm{A}^{+}$ & $\mathrm{R}^{+} \mathrm{A}^{-}$ \\
\hline 1 & $\cdot \mathrm{CH}_{3}$ & $\mathrm{CH}_{2}=\mathrm{CH}_{2}$ & 2.261 & 12.48 & 13.30 \\
\hline 2 & $\cdot \mathrm{CH}_{3}$ & $\mathrm{CH}_{2}=\mathrm{C}\left(\mathrm{CH}_{3}\right)_{2}$ & 2.254 & 9.00 & 16.60 \\
\hline 3 & - $\mathrm{CH}_{2} \mathrm{CH}_{3}$ & $\mathrm{CH}_{2}=\mathrm{CH}_{2}$ & 2.254 & 9.00 & 16.60 \\
\hline 4 & - $\mathrm{CH}_{2} \mathrm{CH}_{3}$ & $\mathrm{CH}_{2}=\mathrm{C}\left(\mathrm{CH}_{3}\right)_{2}$ & 2.242 & 9.00 & 16.60 \\
\hline 5 & $\cdot \mathrm{CH}_{3}$ & $\mathrm{O}=\mathrm{CH}_{2}$ & 1.916 & 13.20 & 12.03 \\
\hline 6 & $\cdot \mathrm{CH}_{3}$ & $\mathrm{O}=\mathrm{C}\left(\mathrm{CH}_{3}\right)_{2}$ & 1.874 & 12.09 & 12.35 \\
\hline 7 & - $\mathrm{CH}_{2} \mathrm{CH}_{3}$ & $\mathrm{O}=\mathrm{CH}_{2}$ & 1.937 & 13.20 & 10.90 \\
\hline 8 & - $\mathrm{CH}_{2} \mathrm{CH}_{3}$ & $\mathrm{O}=\mathrm{C}\left(\mathrm{CH}_{3}\right)_{2}$ & 1.892 & 12.09 & 11.21 \\
\hline 9 & $\cdot \mathrm{OH}$ & $\mathrm{CH}_{2}=\mathrm{CH}_{2}$ & 2.053 & 10.39 & 19.69 \\
\hline 10 & $\cdot \mathrm{OH}$ & $\mathrm{CH}_{2}=\mathrm{C}\left(\mathrm{CH}_{3}\right)_{2}$ & 2.063 & 9.31 & 19.67 \\
\hline 11 & $\cdot \mathrm{OCH}_{3}$ & $\mathrm{CH}_{2}=\mathrm{CH}_{2}$ & 2.006 & 10.09 & 16.62 \\
\hline 12 & $\cdot \mathrm{OCH}_{3}$ & $\mathrm{CH}_{2}=\mathrm{C}\left(\mathrm{CH}_{3}\right)_{2}$ & 2.015 & 9.00 & 16.61 \\
\hline 13 & $\cdot \mathrm{OH}$ & $\mathrm{O}=\mathrm{CH}_{2}$ & 1.572 & 11.11 & 18.43 \\
\hline 14 & $\cdot \mathrm{OH}$ & $\mathrm{O}=\mathrm{C}\left(\mathrm{CH}_{3}\right)_{2}$ & 1.548 & 10.00 & 18.74 \\
\hline 15 & $\cdot \mathrm{OCH}_{3}$ & $\mathrm{O}=\mathrm{CH}_{2}$ & 1.573 & 10.81 & 15.36 \\
\hline 16 & $\cdot \mathrm{OCH}_{3}$ & $\mathrm{O}=\mathrm{C}\left(\mathrm{CH}_{3}\right)_{2}$ & 1.550 & 9.69 & 15.68 \\
\hline 17 & $\cdot \mathrm{SH}$ & $\mathrm{CH}_{2}=\mathrm{CH}_{2}$ & 2.383 & 8.85 & 15.77 \\
\hline 18 & $\bullet \mathrm{SH}$ & $\mathrm{CH}_{2}=\mathrm{C}\left(\mathrm{CH}_{3}\right)_{2}$ & 2.373 & 7.77 & 15.75 \\
\hline 19 & $\cdot \mathrm{SCH}_{3}$ & $\mathrm{CH}_{2}=\mathrm{CH}_{2}$ & 2.373 & 9.27 & 14.49 \\
\hline 20 & $\cdot \mathrm{SCH}_{3}$ & $\mathrm{CH}_{2}=\mathrm{C}\left(\mathrm{CH}_{3}\right)_{2}$ & 2.361 & 8.19 & 14.47 \\
\hline 21 & $\cdot \mathrm{CH}_{3}$ & $\mathrm{~S}=\mathrm{CH}_{2}$ & 2.670 & 11.45 & 10.15 \\
\hline 22 & $\cdot \mathrm{CH}_{3}$ & $\mathrm{~S}=\mathrm{C}\left(\mathrm{CH}_{3}\right)_{2}$ & 2.549 & 10.60 & 10.42 \\
\hline 23 & - $\mathrm{CH}_{2} \mathrm{CH}_{3}$ & $\mathrm{~S}=\mathrm{CH}_{2}$ & 2.752 & 11.45 & 9.01 \\
\hline 24 & - $\mathrm{CH}_{2} \mathrm{CH}_{3}$ & $\mathrm{~S}=\mathrm{C}\left(\mathrm{CH}_{3}\right)_{2}$ & 2.576 & 10.60 & 9.28 \\
\hline 25 & $\cdot \mathrm{SH}$ & $\mathrm{S}=\mathrm{CH}_{2}$ & 2.640 & 7.83 & 12.62 \\
\hline 26 & $\bullet \mathrm{SH}$ & $\mathrm{S}=\mathrm{C}\left(\mathrm{CH}_{3}\right)_{2}$ & 2.516 & 6.98 & 12.89 \\
\hline 27 & $\cdot \mathrm{SCH}_{3}$ & $\mathrm{~S}=\mathrm{CH}_{2}$ & 2.676 & 8.24 & 11.34 \\
\hline 28 & $\cdot \mathrm{SCH}_{3}$ & $\mathrm{~S}=\mathrm{C}\left(\mathrm{CH}_{3}\right)_{2}$ & 2.561 & 7.39 & 11.61 \\
\hline
\end{tabular}

${ }^{a}$ Calculated at the MP2/6-31G(d) level of theory. ${ }^{b}$ Radical addition is calculated for attack on the left-hand side of the substrate. 
Table S2. HOMO and LUMO Orbital Energies of the Reactants ${ }^{a}$

\begin{tabular}{|c|c|c|}
\hline Reactants & HOMO & LUMO \\
\hline $\mathrm{CH}_{3}$. & -0.38364 & 0.15735 \\
\hline $\mathrm{CH}_{3} \mathrm{CH}_{2}$. & -0.35030 & 0.16523 \\
\hline $\mathrm{HO}$ & -0.50384 & 0.13298 \\
\hline $\mathrm{CH}_{3} \mathrm{O}$. & -0.44299 & 0.13247 \\
\hline HS. & -0.37640 & 0.00666 \\
\hline $\mathrm{CH}_{3} \mathrm{~S}$. & -0.34586 & 0.02899 \\
\hline $\mathrm{CH}_{2}=\mathrm{CH}_{2}$ & -0.37006 & 0.17908 \\
\hline $\mathrm{CH}_{2}=\mathrm{C}\left(\mathrm{CH}_{3}\right)_{2}$ & -0.34064 & 0.18821 \\
\hline $\mathrm{O}=\mathrm{CH}_{2}$ & -0.43958 & 0.13387 \\
\hline $\mathrm{O}=\mathrm{C}\left(\mathrm{CH}_{3}\right)_{2}$ & -0.41118 & 0.15717 \\
\hline $\mathrm{S}=\mathrm{CH}_{2}$ & -0.34942 & 0.05349 \\
\hline $\mathrm{S}=\mathrm{C}\left(\mathrm{CH}_{3}\right)_{2}$ & -0.33096 & 0.07479 \\
\hline
\end{tabular}

${ }^{a}$ SCF energies in Hartrees from an MP2/6-31G(d)//MP2/6-31G(d) calculation. 
Table S3. Total Energies at Various Levels of Theory, Thermal Correction, Zero Point Vibrational Energies, High Level Corrections, Entropies and Enthalpies (units are Hartrees unless otherwise noted).

\begin{tabular}{|c|c|c|c|c|c|c|c|c|c|}
\hline $\begin{array}{l}\frac{0}{J} \\
\mathrm{U} \\
\frac{\mathrm{U}}{\mathrm{O}} \\
\mathrm{E}\end{array}$ & 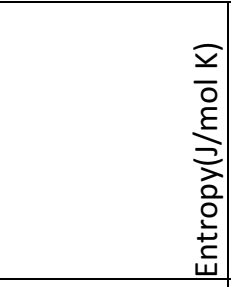 & 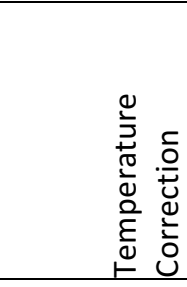 & $\begin{array}{l}\text { 堊 } \\
\text { N }\end{array}$ & $\stackrel{\overrightarrow{\underline{I}}}{\mathrm{u}}$ & 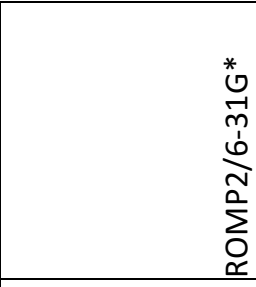 & 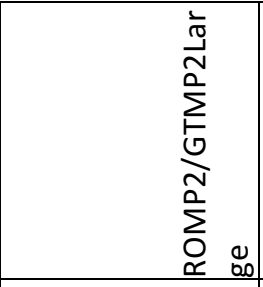 & 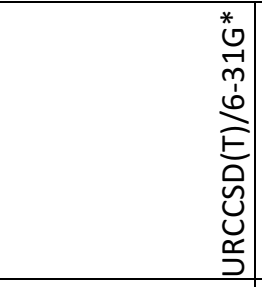 & 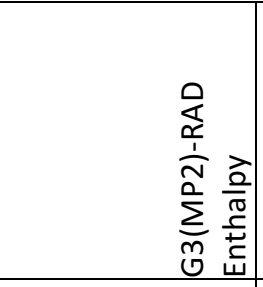 & 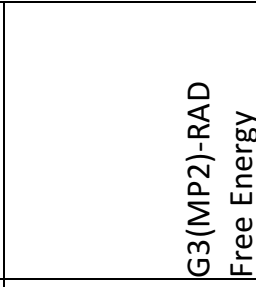 \\
\hline $\mathrm{CH}_{3}$ & 210.72230 & 0.00409 & 0.02952 & -0.03221 & -39.55891 & -39.73057 & -39.69097 & -39.75159 & \begin{tabular}{|l|}
-39.77552 \\
\end{tabular} \\
\hline $\mathrm{CH}_{3} \mathrm{CH}_{2}$ & 252.78310 & 0.00480 & 0.05943 & -0.06045 & -78.59691 & \begin{tabular}{|l|}
-78.94879 \\
\end{tabular} & -78.87471 & -78.98412 & \begin{tabular}{|l|}
-79.01283 \\
\end{tabular} \\
\hline $\mathrm{HO}$ & 178.22710 & 0.00330 & \begin{tabular}{|l|}
0.00824 \\
\end{tabular} & -0.03221 & -75.38186 & -75.61470 & -75.53709 & -75.65139 & \begin{tabular}{|l|}
-75.67163 \\
\end{tabular} \\
\hline $\mathrm{CH}_{3} \mathrm{O}$ & 236.40500 & 0.00391 & 0.03714 & -0.06045 & -114.42035 & -114.82565 & -114.72131 & -114.88021 & -114.90706 \\
\hline $\mathrm{HS}$ & 192.01310 & 0.00330 & 0.00612 & -0.03221 & -398.06429 & -398.25184 & -398.18455 & -398.29695 & -398.31876 \\
\hline $\mathrm{CH}_{3} \mathrm{~S}$ & 249.36690 & 0.00408 & 0.03583 & -0.06045 & -437.10128 & -437.46946 & -437.36935 & -437.52803 & -437.55634 \\
\hline $\mathrm{CH}_{2} \mathrm{CH}_{2}$ & 218.74330 & 0.00398 & 0.05032 & -0.05648 & -78.03098 & \begin{tabular}{|l|}
-78.39088 \\
\end{tabular} & -78.32192 & -78.42996 & \begin{tabular}{|l|}
-78.45480 \\
\end{tabular} \\
\hline $\mathrm{CH}_{2}=\mathrm{C}\left(\mathrm{CH}_{3}\right)_{2}$ & 294.23500 & 0.00620 & \begin{tabular}{|l|}
0.10695 \\
\end{tabular} & -0.11296 & \begin{tabular}{|l|}
-156.10969 \\
\end{tabular} & \begin{tabular}{|l|}
-156.83662 \\
\end{tabular} & -156.69493 & \begin{tabular}{|l|}
-156.90401 \\
\end{tabular} & -156.93743 \\
\hline $\mathrm{O}=\mathrm{C}(\mathrm{H})_{2}$ & 224.39780 & 0.00381 & 0.02638 & -0.05648 & -113.86354 & \begin{tabular}{|l|}
-114.30003 \\
\end{tabular} & -114.19057 & -114.34915 & -114.37463 \\
\hline $\mathrm{O}=\mathrm{C}\left(\mathrm{CH}_{3}\right)_{2}$ & 302.19220 & 0.00625 & 0.08309 & -0.11296 & \begin{tabular}{|l|}
-191.95971 \\
\end{tabular} & \begin{tabular}{|l|}
-192.75904 \\
\end{tabular} & -192.57811 & -192.83686 & -192.87118 \\
\hline $\mathrm{S}=\mathrm{C}(\mathrm{H})_{2}$ & 230.52720 & 0.00386 & 0.02463 & -0.05648 & -436.50588 & -436.88527 & -436.79099 & -436.94914 & -436.97532 \\
\hline $\mathrm{S}=\mathrm{C}\left(\mathrm{CH}_{3}\right)_{2}$ & 306.28080 & 0.00628 & 0.08150 & -0.11296 & -514.59349 & -515.34000 & -515.17163 & -515.43092 & -515.46571 \\
\hline Hydrogen radical & 114.60640 & 0.00236 & 0.00000 & -0.00189 & -0.49823 & -0.49982 & -0.49823 & -0.49935 & -0.51236 \\
\hline $\mathrm{CH}_{3}-\mathrm{H}$ & 206.59460 & 0.00380 & 0.04482 & -0.03765 & -40.19506 & -40.40424 & -40.35586 & -40.41657 & -40.44003 \\
\hline $\mathrm{CH}_{3} \mathrm{CH}_{2}-\mathrm{H}$ & 226.57330 & 0.00437 & 0.07464 & -0.06589 & -79.22853 & -79.61764 & -79.53440 & -79.64418 & \begin{tabular}{|l|}
-79.66991 \\
\end{tabular} \\
\hline $\mathrm{HO}-\mathrm{H}$ & 188.83530 & 0.00378 & 0.02077 & -0.03765 & -76.00980 & -76.31476 & -76.20784 & -76.33885 & \begin{tabular}{|l|}
-76.36030 \\
\end{tabular} \\
\hline $\mathrm{CH}_{3} \mathrm{O}-\mathrm{H}$ & 237.03990 & 0.00422 & 0.05086 & -0.06589 & -115.03412 & -115.50793 & -115.37464 & -115.54726 & -115.57417 \\
\hline $\mathrm{HS}-\mathrm{H}$ & 205.47910 & 0.00379 & 0.01520 & -0.03765 & -398.66710 & -398.89691 & -398.81337 & -398.94053 & -398.96387 \\
\hline $\mathrm{CH}_{3} \mathrm{~S}-\mathrm{H}$ & 251.77320 & 0.00446 & \begin{tabular}{|l|}
0.04621 \\
\end{tabular} & -0.06589 & \begin{tabular}{|l|}
-437.69998 \\
\end{tabular} & \begin{tabular}{|l|}
-438.10920 \\
\end{tabular} & -437.99320 & -438.16495 & -438.19354 \\
\hline $\mathrm{CH}_{3}-\mathrm{CH}_{3}$ & 226.57330 & 0.00437 & \begin{tabular}{|l|}
0.07464 \\
\end{tabular} & -0.06589 & -79.22853 & \begin{tabular}{|l|}
-79.61764 \\
\end{tabular} & -79.53440 & -79.64418 & \begin{tabular}{|l|}
-79.66991 \\
\end{tabular} \\
\hline $\mathrm{CH}_{3} \mathrm{CH}_{2}-\mathrm{CH}_{2} \mathrm{CH}_{3}$ & 302.75600 & 0.00658 & 0.13159 & -0.12237 & -157.29789 & -158.05252 & -157.89791 & -158.10859 & -158.14298 \\
\hline $\mathrm{HO}-\mathrm{OH}$ & 233.97940 & 0.00421 & 0.02540 & -0.06589 & -150.76003 & -151.32249 & -151.15124 & -151.37988 & -151.40645 \\
\hline $\mathrm{CH}_{3} \mathrm{O}-\mathrm{OCH}_{3}$ & 302.28640 & 0.00638 & 0.08254 & -0.12237 & -228.82313 & -229.73438 & -229.50581 & -229.82275 & -229.85708 \\
\hline $\mathrm{HS}-\mathrm{SH}$ & 250.96670 & 0.00432 & 0.01851 & -0.06589 & \begin{tabular}{|l|}
-796.17407 \\
\end{tabular} & \begin{tabular}{|l|}
-796.60738 \\
\end{tabular} & -796.45361 & -796.69271 & -796.72121 \\
\hline $\mathrm{CH} 3 \mathrm{~S}-\mathrm{SCH} 3$ & 323.76700 & 0.00724 & \begin{tabular}{|l|}
0.07757 \\
\end{tabular} & -0.12237 & \begin{tabular}{|l|}
-874.24717 \\
\end{tabular} & \begin{tabular}{|l|}
-875.04724 \\
\end{tabular} & -874.82541 & -875.15699 & -875.19376 \\
\hline Cl radical* & 158.83930 & 0.00236 & 0.00000 & -0.03020 & \begin{tabular}{|l|}
-459.44796 \\
\end{tabular} & \begin{tabular}{|l|}
-459.63877 \\
\end{tabular} & -459.57046 & -459.68551 & -459.70355 \\
\hline $\mathrm{Cl}-\mathrm{Cl}$ & 222.79750 & 0.00349 & \begin{tabular}{|l|}
0.00120 \\
\end{tabular} & -0.06589 & \begin{tabular}{|l|}
-918.91255 \\
\end{tabular} & \begin{tabular}{|l|}
-919.36706 \\
\end{tabular} & -919.20547 & -919.46233 & -919.48763 \\
\hline $\mathrm{CH}_{3}-\mathrm{Cl}$ & 233.47990 & 0.00392 & 0.03787 & -0.06589 & -499.09290 & -499.50974 & -499.38971 & -499.56898 & -499.59550 \\
\hline
\end{tabular}




\begin{tabular}{|c|c|c|c|c|c|c|c|c|c|}
\hline $\mathrm{CH}_{3} \mathrm{CH}_{2}-\mathrm{Cl}$ & 272.74670 & 0.00487 & 0.06665 & -0.09413 & -538.13115 & -538.73090 & -538.57504 & -538.80453 & -538.83550 \\
\hline $\mathrm{HO}-\mathrm{Cl}$ & 236.44030 & 0.00388 & 0.01275 & -0.06589 & -534.84009 & -535.34809 & -535.18345 & -535.42397 & -535.45082 \\
\hline $\mathrm{CH}_{3} \mathrm{O} \_\mathrm{Cl}$ & 269.82870 & 0.00475 & 0.04174 & -0.09413 & -573.86975 & -574.55217 & -574.35868 & -574.64325 & -574.67389 \\
\hline $\mathrm{HS}-\mathrm{Cl}$ & 250.43660 & 0.00401 & 0.00945 & -0.06589 & -857.54982 & -857.99289 & -857.83452 & -858.08322 & -858.11166 \\
\hline $\mathrm{CH}_{3} \mathrm{~S}-\mathrm{Cl}$ & 285.94290 & 0.00517 & 0.03941 & -0.09413 & -896.59026 & -897.21550 & -897.02332 & -897.31795 & -897.35042 \\
\hline $\mathrm{CH}_{3}-\mathrm{CH}_{3}$ & 226.57330 & 0.00437 & 0.07464 & -0.06589 & -79.22853 & -79.61764 & -79.53440 & -79.64418 & -79.66991 \\
\hline $\mathrm{CH}_{3} \mathrm{CH}_{2}-\mathrm{CH}_{3}$ & 266.05530 & 0.00535 & 0.10322 & -0.09413 & -118.26326 & -118.83499 & -118.71611 & -118.87631 & -118.90653 \\
\hline $\mathrm{HO}-\mathrm{CH}_{3}$ & 237.03990 & 0.00422 & 0.05086 & -0.06589 & -115.03412 & -115.50793 & -115.37464 & -115.54726 & -115.57417 \\
\hline $\mathrm{CH}_{3} \mathrm{O}-\mathrm{CH}_{3}$ & 262.54060 & 0.00518 & 0.07949 & -0.09413 & -154.06324 & -154.70993 & -154.54900 & -154.76492 & -154.79473 \\
\hline $\mathrm{HS}-\mathrm{CH}_{3}$ & 251.77320 & 0.00446 & 0.04621 & -0.06589 & -437.69998 & -438.10920 & -437.99320 & -438.16495 & -438.19354 \\
\hline $\mathrm{CH}_{3} \mathrm{~S}-\mathrm{CH}_{3}$ & 281.09000 & 0.00570 & 0.07588 & -0.09413 & -476.73486 & -477.32638 & -477.17686 & -477.39468 & -477.42660 \\
\hline TS1 & 290.24860 & 0.00594 & 0.08460 & -0.08869 & -117.57440 & -118.10852 & -117.99940 & -118.16772 & -118.20068 \\
\hline TS2 & 355.38720 & 0.00870 & 0.14064 & -0.14516 & -195.65126 & -196.55568 & -196.37332 & -196.64277 & -196.68312 \\
\hline TS3 & 321.32840 & 0.00704 & 0.11377 & -0.11693 & -156.61146 & -157.32914 & -157.18447 & -157.40220 & -157.43869 \\
\hline TS4 & 385.43680 & 0.00982 & 0.16976 & -0.17340 & -234.68748 & -235.77585 & -235.55821 & -235.87695 & -235.92072 \\
\hline TS5 & 285.44340 & 0.00567 & 0.06086 & -0.08869 & -153.38522 & -154.00162 & -153.85059 & -154.07027 & -154.10269 \\
\hline TS6 & 314.46380 & 0.00672 & 0.08991 & -0.11693 & -192.42393 & -193.22787 & -193.03928 & -193.30958 & -193.34529 \\
\hline TS7 & 358.27310 & 0.00853 & 0.11698 & -0.14516 & -231.47219 & -232.45844 & -232.23497 & -232.55463 & -232.59532 \\
\hline TS8 & 394.39370 & 0.00984 & 0.14561 & -0.17340 & -270.51259 & -271.68134 & -271.42289 & -271.79189 & -271.83668 \\
\hline TS9 & 292.20820 & 0.00567 & 0.06234 & -0.08869 & -153.40002 & -154.00394 & -153.85661 & -154.08000 & -154.11318 \\
\hline TS10 & 346.71090 & 0.00819 & 0.11884 & -0.14516 & -231.47634 & -232.45318 & -232.23196 & -232.55709 & -232.59647 \\
\hline TS11 & 312.46200 & 0.00659 & 0.09158 & -0.11693 & -192.42836 & -193.20650 & -193.03298 & -193.29944 & -193.33493 \\
\hline TS12 & 376.33440 & 0.00934 & 0.14770 & -0.17340 & -270.50556 & -271.65769 & -271.40969 & -271.77810 & -271.82084 \\
\hline TS13 & 271.20760 & 0.00507 & 0.03849 & -0.08869 & -189.17042 & -189.86312 & -189.67502 & -189.94982 & -189.98062 \\
\hline TS14 & 342.73740 & 0.00791 & 0.09476 & -0.14516 & -267.25618 & -268.31730 & -268.05724 & -268.43116 & -268.47008 \\
\hline TS15 & 307.58420 & 0.00630 & 0.06696 & -0.11693 & -228.20304 & -229.06990 & -228.85297 & -229.17152 & -229.20645 \\
\hline TS16 & 377.29940 & 0.00922 & 0.12313 & -0.17340 & -306.28829 & -307.52408 & -307.23547 & -307.65313 & -307.69597 \\
\hline TS17 & 292.98940 & 0.00560 & 0.05955 & -0.08869 & -476.08640 & -476.64468 & -476.50197 & -476.72641 & -476.75968 \\
\hline TS18 & 357.06960 & 0.00839 & 0.11565 & -0.14516 & -554.16538 & -555.09689 & -554.87901 & -555.20550 & -555.24605 \\
\hline TS19 & 331.07830 & 0.00703 & 0.08950 & -0.11693 & -515.11917 & -515.85939 & -515.68385 & -515.95365 & -515.99124 \\
\hline TS20 & 392.91780 & 0.00980 & 0.14563 & -0.17340 & -593.19680 & -594.31104 & -594.06055 & -594.43209 & -594.47671 \\
\hline TS21 & 316.86390 & 0.00657 & 0.05749 & -0.08869 & -476.06427 & -476.61484 & -476.47822 & -476.69781 & -476.73379 \\
\hline As22 & 380.46120 & 0.00922 & 0.11419 & -0.14516 & -554.14541 & -555.06782 & -554.85707 & -555.17856 & -555.22177 \\
\hline TS23 & 352.73030 & 0.00781 & 0.08663 & -0.11693 & -515.10217 & -515.83658 & -515.66422 & -515.93332 & -515.97337 \\
\hline TS24 & 411.87190 & 0.01039 & 0.14329 & -0.17340 & -593.18299 & -594.29011 & -594.04337 & -594.41464 & -594.46141 \\
\hline TS25 & 305.98110 & 0.00580 & 0.03329 & -0.08869 & -834.56459 & -835.14485 & -834.97497 & -835.25420 & -835.28894 \\
\hline TS26 & 373.05730 & 0.00862 & 0.08961 & -0.14516 & -912.64704 & -913.60457 & -913.35651 & -913.74042 & -913.78279 \\
\hline TS27 & 352.47570 & 0.00747 & 0.06301 & -0.11693 & -873.60240 & -874.36027 & -874.15745 & -874.48119 & -874.52122 \\
\hline
\end{tabular}




\begin{tabular}{|c|c|c|c|c|c|c|c|c|c|}
\hline TS28 & 414.80420 & 0.01024 & 0.11938 & -0.17340 & -951.68320 & -952.81851 & -952.53880 & -952.96645 & -953.01356 \\
\hline Product1 & 282.90250 & 0.00572 & 0.08821 & -0.08869 & -117.63133 & -118.16564 & -118.05580 & -118.21569 & -118.24782 \\
\hline Product2 & 348.52740 & 0.00827 & 0.14543 & -0.14516 & -195.70753 & -196.60959 & -196.42961 & -196.68860 & -196.72818 \\
\hline Product3 & 314.16450 & 0.00694 & 0.11657 & -0.11693 & -156.66603 & -157.38322 & -157.23766 & -157.44804 & -157.48371 \\
\hline Product4 & 378.94000 & 0.00955 & 0.17373 & -0.17340 & -234.74222 & -235.82749 & -235.61176 & -235.92125 & -235.96428 \\
\hline Product5 & 273.45460 & 0.00523 & 0.06583 & -0.08869 & -153.43589 & -154.04912 & -153.89716 & -154.11162 & -154.14267 \\
\hline Product6 & 304.59690 & 0.00637 & 0.09416 & -0.11693 & -192.47594 & -193.27153 & -193.08426 & -193.34886 & -193.38345 \\
\hline Product7 & 334.96090 & 0.00775 & 0.12243 & -0.14516 & -231.51669 & -232.49535 & -232.27376 & -232.58769 & -232.62572 \\
\hline Product8 & 365.37420 & 0.00903 & 0.15064 & -0.17340 & -270.55655 & -271.71776 & -271.46101 & -271.82492 & -271.86642 \\
\hline Product9 & 274.29950 & 0.00528 & 0.06477 & -0.08869 & -153.44175 & -154.05985 & -153.90127 & -154.12265 & -154.15380 \\
\hline Product10 & 347.06800 & 0.00808 & 0.12176 & -0.14516 & -231.51818 & -232.50375 & -232.27519 & -232.59571 & -232.63512 \\
\hline Product11 & 305.85240 & 0.00650 & 0.09308 & -0.11693 & -192.46874 & -193.26027 & -193.07396 & -193.33878 & -193.37351 \\
\hline Product12 & 376.67420 & 0.00934 & 0.15010 & -0.17340 & -270.54725 & -271.70605 & -271.44943 & -271.81339 & -271.85616 \\
\hline Product13 & 271.90050 & 0.00515 & 0.04033 & -0.08869 & -189.16192 & -189.86395 & -189.67374 & -189.94496 & -189.97583 \\
\hline Product14 & 335.41610 & 0.00775 & 0.09694 & -0.14516 & -267.24473 & -268.31253 & -268.05265 & -268.42312 & -268.46121 \\
\hline Product15 & 307.28470 & 0.00622 & 0.06909 & -0.11693 & -228.19310 & -229.07029 & -228.85150 & -229.16683 & -229.20173 \\
\hline Product16 & 365.82100 & 0.00892 & 0.12554 & -0.17340 & -306.27595 & -307.51931 & -307.23126 & -307.64538 & -307.68692 \\
\hline Product17 & 290.29100 & 0.00570 & 0.06042 & -0.08869 & -476.10457 & -476.66012 & -476.51788 & -476.73896 & -476.77193 \\
\hline Product18 & 355.62470 & 0.00838 & 0.11721 & -0.14516 & -554.18225 & -555.10708 & -554.89376 & -555.21486 & -555.25524 \\
\hline Product19 & 325.48660 & 0.00713 & 0.08987 & -0.11693 & -515.13850 & -515.87706 & -515.70113 & -515.96839 & -516.00535 \\
\hline Product20 & 392.35420 & 0.00987 & 0.14659 & -0.17340 & -593.21452 & -594.32339 & -594.07626 & -594.44372 & -594.48827 \\
\hline Product21 & 292.52510 & 0.00582 & 0.06180 & -0.08869 & -476.10760 & -476.66863 & -476.52628 & -476.74527 & -476.77849 \\
\hline Product22 & 355.12640 & 0.00842 & 0.11899 & -0.14516 & -554.18292 & -555.11094 & -554.89818 & -555.21589 & -555.25622 \\
\hline Product23 & 318.80950 & 0.00685 & 0.09054 & -0.11693 & -515.14285 & -515.88794 & -515.70989 & -515.97888 & -516.01508 \\
\hline Product24 & 381.73300 & 0.00961 & 0.14752 & -0.17340 & -593.21776 & -594.33065 & -594.08199 & -594.44991 & -594.49326 \\
\hline Product25 & 302.82300 & 0.00602 & 0.03346 & -0.08869 & -834.58120 & -835.16584 & -834.98636 & -835.27265 & -835.30704 \\
\hline Product26 & 369.17050 & 0.00870 & 0.09061 & -0.14516 & -912.65913 & -913.61115 & -913.36209 & -913.74683 & -913.78875 \\
\hline Product27 & 333.24350 & 0.00727 & 0.06350 & -0.11693 & -873.61869 & -874.38669 & -874.17333 & -874.50511 & -874.54296 \\
\hline Product28 & 402.58970 & 0.01023 & 0.12017 & -0.17340 & -951.69468 & -952.83078 & -952.54757 & -952.97836 & -953.02408 \\
\hline Product1-H & 266.05530 & 0.00535 & 0.10322 & -0.09413 & -118.26326 & -118.83499 & -118.71611 & -118.87631 & -118.90653 \\
\hline Product2-H & 328.75130 & 0.00774 & 0.15955 & -0.15061 & -196.33105 & -197.27240 & -197.08092 & -197.34326 & -197.38059 \\
\hline Product3-H & 296.92760 & 0.00658 & 0.13159 & -0.12237 & -157.29788 & -158.05252 & -157.89791 & -158.10860 & -158.14232 \\
\hline Product4-H & 359.68620 & 0.00905 & 0.18785 & -0.17885 & -235.36545 & -236.48996 & -236.26274 & -236.57555 & -236.61639 \\
\hline Product5-H & 268.29970 & 0.00518 & 0.07950 & -0.09413 & -154.06323 & -154.70993 & -154.54899 & -154.76491 & -154.79538 \\
\hline Product6-H & 299.26960 & 0.00637 & 0.10780 & -0.12237 & -193.10322 & -193.93237 & -193.73612 & -194.00214 & -194.03612 \\
\hline Product7-H & 329.06530 & 0.00762 & 0.13558 & -0.15061 & -232.13976 & -233.15432 & -232.92240 & -233.23918 & -233.27655 \\
\hline Product8-H & 361.55360 & 0.00890 & 0.16382 & -0.17885 & -271.17955 & -272.37671 & -272.10963 & -272.47633 & -272.51739 \\
\hline Product9-H & 267.13040 & 0.00510 & 0.07948 & -0.09413 & -154.07414 & -154.72979 & -154.56176 & -154.78399 & -154.81432 \\
\hline
\end{tabular}




\begin{tabular}{|c|c|c|c|c|c|c|c|c|c|}
\hline Product10-H & 323.26660 & 0.00748 & 0.13585 & -0.15061 & -232.14408 & -233.16784 & -232.92829 & -233.25155 & -233.28826 \\
\hline Product11-H & 299.28020 & 0.00637 & 0.10780 & -0.12237 & -193.10323 & -193.93236 & -193.73612 & -194.00214 & -194.03613 \\
\hline Product12-H & 355.60020 & 0.00884 & 0.16409 & -0.17885 & -271.17290 & -272.37047 & -272.10255 & -272.46973 & -272.51011 \\
\hline Product13-H & 270.62540 & 0.00527 & 0.05393 & -0.09413 & -189.79168 & -190.52815 & -190.32814 & -190.60115 & -190.63188 \\
\hline Product14-H & 324.75960 & 0.00754 & 0.11021 & -0.15061 & -267.87015 & -268.97459 & -268.70342 & -269.07720 & -269.11408 \\
\hline Product15-H & 302.21450 & 0.00638 & 0.08254 & -0.12237 & -228.82312 & -229.73437 & -229.50581 & -229.82275 & -229.85707 \\
\hline Product16-H & 356.25020 & 0.00873 & 0.13872 & -0.17885 & -306.90127 & -308.18090 & -307.88142 & -308.29890 & -308.33935 \\
\hline Product17-H & 281.53690 & 0.00545 & 0.07494 & -0.09413 & -476.73571 & -477.32862 & -477.17670 & -477.39872 & -477.43069 \\
\hline Product18-H & 337.72640 & 0.00791 & 0.13116 & -0.15061 & -554.80437 & -555.76745 & -555.54278 & -555.86687 & -555.90522 \\
\hline Product19-H & 317.09730 & 0.00688 & 0.10446 & -0.12237 & -515.76922 & -516.54520 & -516.35952 & -516.62781 & -516.66382 \\
\hline Product20-H & 373.52480 & 0.00937 & 0.16066 & -0.17885 & -593.83777 & -594.98437 & -594.72592 & -595.09624 & -595.13866 \\
\hline Product21-H & 286.83320 & 0.00570 & 0.07588 & -0.09413 & -476.73486 & -477.32638 & -477.17686 & -477.39468 & -477.42725 \\
\hline Product22-H & 344.00010 & 0.00815 & 0.13240 & -0.15061 & -554.80414 & -555.76681 & -555.54405 & -555.86383 & -555.90290 \\
\hline Product23-H & 317.10400 & 0.00688 & 0.10446 & -0.12237 & -515.76923 & -516.54520 & -516.35952 & -516.62781 & -516.66382 \\
\hline Product24-H & 375.90580 & 0.00944 & 0.16097 & -0.17885 & -593.83894 & -594.98558 & -594.72668 & -595.09673 & -595.13941 \\
\hline Product25-H & 292.87600 & 0.00566 & 0.04813 & -0.09413 & -835.21099 & -835.82737 & -835.63957 & -835.92497 & -835.95823 \\
\hline Product26-H & 346.76380 & 0.00809 & 0.10471 & -0.15061 & -913.28182 & -914.27003 & -914.00874 & -914.39604 & -914.43542 \\
\hline Product27-H & 329.52700 & 0.00724 & 0.07757 & -0.12237 & -874.24717 & -875.04724 & -874.82541 & -875.15699 & -875.19442 \\
\hline Product28-H & 383.02400 & 0.00971 & 0.13411 & -0.17885 & -952.31787 & -953.48998 & -953.19474 & -953.62812 & -953.67162 \\
\hline Product1- $\mathrm{CH}_{3}$ & 296.92760 & 0.00658 & 0.13159 & -0.12237 & -157.29788 & -158.05252 & -157.89791 & -158.10860 & -158.14232 \\
\hline Product2- $\mathrm{CH}_{3}$ & 350.69680 & 0.00890 & 0.18725 & -0.17885 & -235.36359 & -236.49441 & -236.26528 & -236.57985 & -236.61967 \\
\hline Product3- $\mathrm{CH}_{3}$ & 327.88440 & 0.00785 & 0.15992 & -0.15061 & -196.33244 & -197.27002 & -197.07970 & -197.34084 & -197.37808 \\
\hline Product4- $\mathrm{CH}_{3}$ & 381.93770 & 0.01022 & 0.21553 & -0.20709 & -274.39781 & -275.71200 & -275.44712 & -275.81213 & -275.85550 \\
\hline Product5- $\mathrm{CH}_{3}$ & 299.27760 & 0.00637 & 0.10780 & -0.12237 & -193.10323 & -193.93236 & -193.73612 & -194.00214 & -194.03613 \\
\hline Product6- $\mathrm{CH}_{3}$ & 324.65130 & 0.00762 & 0.13606 & -0.15061 & -232.14311 & -233.15473 & -232.92327 & -233.23928 & -233.27615 \\
\hline Product7- $\mathrm{CH}_{3}$ & 370.79750 & 0.00892 & 0.16308 & -0.17885 & -271.17366 & -272.37650 & -272.10787 & -272.47625 & -272.51836 \\
\hline Product8- $\mathrm{CH}_{3}$ & 380.92710 & 0.01006 & 0.19145 & -0.20709 & -310.21327 & -311.59894 & -311.29513 & -311.71344 & -311.75669 \\
\hline Product9- $\mathrm{CH}_{3}$ & 299.25950 & 0.00638 & 0.10780 & -0.12237 & -193.10890 & -193.94719 & -193.74298 & -194.01607 & -194.05006 \\
\hline Product10- $\mathrm{CH}_{3}$ & 344.38850 & 0.00863 & 0.16355 & -0.17885 & -271.17818 & -272.39073 & -272.11401 & -272.48903 & -272.52814 \\
\hline Product11- $\mathrm{CH}_{3}$ & 330.73440 & 0.00766 & 0.13616 & -0.15061 & -232.13778 & -233.14943 & -232.91764 & -233.23395 & -233.27150 \\
\hline Product12- $\mathrm{CH}_{3}$ & 376.56640 & 0.01002 & 0.19173 & -0.20709 & -310.20721 & -311.59352 & -311.28861 & -311.70733 & -311.75010 \\
\hline Product13- $\mathrm{CH}_{3}$ & 300.74180 & 0.00644 & 0.08232 & -0.12237 & -228.83145 & -229.75055 & -229.51487 & -229.83811 & -229.87227 \\
\hline Product14- $\mathrm{CH}_{3}$ & 344.99170 & 0.00867 & 0.13784 & -0.17885 & -306.90669 & -308.19885 & -307.89172 & -308.31636 & -308.35553 \\
\hline Product15- $\mathrm{CH}_{3}$ & 331.29510 & 0.00746 & 0.11097 & -0.15061 & -267.86227 & -268.95657 & -268.69317 & -269.05973 & -269.09735 \\
\hline Product16- $\mathrm{CH}_{3}$ & 376.21100 & 0.00994 & 0.16628 & -0.20709 & -345.93751 & -347.40554 & -347.06960 & -347.53833 & -347.58105 \\
\hline Product17- $-\mathrm{CH}_{3}$ & 312.27150 & 0.00673 & 0.10329 & -0.12237 & -515.77063 & -516.54658 & -516.35902 & -516.63127 & -516.66673 \\
\hline Product18- $\mathrm{CH}_{3}$ & 356.23350 & 0.00899 & 0.15893 & -0.17885 & -593.83727 & -594.99046 & -594.72786 & -595.10430 & -595.14476 \\
\hline Product19- $\mathrm{CH}_{3}$ & 347.86070 & 0.00817 & 0.13280 & -0.15061 & -554.80401 & -555.76312 & -555.54183 & -555.86031 & -555.89982 \\
\hline
\end{tabular}




\begin{tabular}{|c|c|c|c|c|c|c|c|c|c|}
\hline Product20- $\mathrm{CH}_{3}$ & 397.19950 & 0.01063 & 0.18831 & -0.20709 & -632.87047 & -634.20630 & -633.91015 & -634.33251 & -634.37761 \\
\hline Product21- $\mathrm{CH}_{3}$ & 318.94510 & 0.00696 & 0.10447 & -0.12237 & -515.76992 & -516.54518 & -516.35946 & -516.62764 & -516.66385 \\
\hline Product22- $\mathrm{CH}_{3}$ & 369.51370 & 0.00938 & 0.16003 & -0.17885 & -593.83687 & -594.98953 & -594.72887 & -595.10095 & -595.14291 \\
\hline Product23- $\mathrm{CH}_{3}$ & 349.50220 & 0.00815 & 0.13304 & -0.15061 & -554.80413 & -555.76397 & -555.54213 & -555.86071 & -555.90040 \\
\hline Product24- $\mathrm{CH}_{3}$ & 400.46830 & 0.01067 & 0.18861 & -0.20709 & -632.87142 & -634.20823 & -633.91140 & -634.33374 & -634.37922 \\
\hline Product25- $\mathrm{CH}_{3}$ & 319.95670 & 0.00676 & 0.07679 & -0.12237 & -874.24664 & -875.04801 & -874.82379 & -875.15978 & -875.19611 \\
\hline Product26- $\mathrm{CH}_{3}$ & 365.52160 & 0.00924 & 0.13240 & -0.17885 & -952.31566 & -953.49452 & -953.19510 & -953.63479 & -953.67630 \\
\hline Product27- $\mathrm{CH}_{3}$ & 356.44150 & 0.00839 & 0.10617 & -0.15061 & -913.28263 & -914.26776 & -914.00959 & -914.39166 & -914.43214 \\
\hline Product28- $\mathrm{CH}_{3}$ & 398.90630 & 0.01082 & 0.16185 & -0.20709 & -991.35089 & -992.71429 & -992.38070 & -992.86668 & -992.91198 \\
\hline Product1-Cl & 303.56000 & 0.00614 & 0.09504 & -0.12237 & -577.16608 & -577.94865 & -577.75717 & -578.03685 & -578.07132 \\
\hline Product2-Cl & 348.97840 & 0.00851 & 0.15060 & -0.17885 & -655.23705 & -656.39595 & -656.12977 & -656.51337 & -656.55300 \\
\hline Product3-Cl & 334.64470 & 0.00743 & 0.12340 & -0.15061 & -616.20067 & -617.16616 & -616.93896 & -617.26905 & -617.30706 \\
\hline Product4-Cl & 379.75040 & 0.00985 & 0.17888 & -0.20709 & -694.27131 & -695.61356 & -695.31158 & -695.74565 & -695.78878 \\
\hline Product5-Cl & 299.02670 & 0.00582 & 0.07140 & -0.12237 & -612.96994 & -613.82921 & -613.59546 & -613.93108 & -613.96503 \\
\hline Product6-Cl & 330.40030 & 0.00707 & 0.09964 & -0.15061 & -652.01004 & -653.05183 & -652.78276 & -653.16850 & -653.20602 \\
\hline Product7-Cl & 345.27150 & 0.00830 & 0.12665 & -0.17885 & -691.04766 & -692.27985 & -691.97400 & -692.41131 & -692.45052 \\
\hline Product8-Cl & 375.53230 & 0.00959 & 0.15485 & -0.20709 & -730.08751 & -731.50260 & -731.16144 & -731.64882 & -731.69147 \\
\hline Product9-Cl & 295.18670 & 0.00565 & 0.07150 & -0.12237 & -612.97747 & -613.84429 & -613.60373 & -613.94544 & -613.97896 \\
\hline Product10-Cl & 342.44880 & 0.00816 & 0.12700 & -0.17885 & -691.05257 & -692.29304 & -691.97955 & -692.42334 & -692.46223 \\
\hline Product11-Cl & 332.04570 & 0.00725 & 0.09954 & -0.15061 & -652.00457 & -653.04377 & -652.77529 & -653.16059 & -653.19830 \\
\hline Product12-Cl & 377.85710 & 0.00970 & 0.15505 & -0.20709 & -730.07933 & \begin{tabular}{|l|}
-731.49312 \\
\end{tabular} & -731.15169 & -731.63895 & -731.68186 \\
\hline Product13-Cl & 294.93640 & 0.00563 & 0.04594 & -0.12237 & -648.69712 & -649.64592 & -649.37362 & -649.76597 & -649.79946 \\
\hline Product14-Cl & 342.50890 & 0.00817 & 0.10116 & -0.17885 & -726.77747 & -728.09910 & -727.75465 & -728.24840 & -728.28729 \\
\hline Product15-Cl & 326.75390 & 0.00690 & 0.07436 & -0.15061 & -687.72766 & -688.85210 & -688.55088 & -688.98768 & -689.02479 \\
\hline Product16-Cl & 372.57990 & 0.00950 & 0.12954 & -0.20709 & -765.80693 & \begin{tabular}{|l|}
-767.30493 \\
\end{tabular} & -766.93141 & -767.46967 & -767.51198 \\
\hline Product17-Cl & 312.59490 & 0.00629 & 0.06664 & -0.12237 & -935.63706 & -936.44184 & -936.21677 & -936.55885 & -936.59435 \\
\hline Product18-Cl & 356.83250 & 0.00866 & 0.12208 & -0.17885 & -1013.70830 & -1014.89014 & -1014.59023 & -1015.03607 & -1015.07660 \\
\hline Product19-Cl & 348.82360 & 0.00777 & 0.09612 & -0.15061 & -974.67111 & -975.65875 & -975.40007 & -975.78826 & -975.82787 \\
\hline Product20-Cl & 389.06270 & 0.01013 & 0.15172 & -0.20709 & -1052.74127 & -1054.10652 & -1053.77311 & -1054.26489 & -1054.30908 \\
\hline Product21-Cl & 315.92770 & 0.00639 & 0.06761 & -0.12237 & -935.63197 & -936.43863 & -936.21445 & -936.55394 & -936.58981 \\
\hline Product22-Cl & 362.33560 & 0.00894 & 0.12319 & -0.17885 & -1013.70369 & -1014.88682 & -1014.58798 & -1015.03053 & -1015.07168 \\
\hline Product23-Cl & 347.57530 & 0.00759 & 0.09621 & -0.15061 & -974.66647 & -975.65792 & -975.39739 & -975.78744 & -975.82691 \\
\hline Product24-Cl & 393.32200 & 0.01026 & 0.15175 & -0.20709 & -1052.73843 & -1054.10565 & -1053.77067 & -1054.26347 & -1054.30813 \\
\hline Product25-Cl & 321.16590 & 0.00633 & 0.03983 & -0.12237 & -1294.10632 & -1294.93859 & -1294.67586 & -1295.08315 & -1295.11962 \\
\hline Product26-Cl & 366.69700 & 0.00893 & 0.09536 & -0.17885 & -1372.17958 & -1373.38921 & -1373.05145 & -1373.56179 & -1373.60344 \\
\hline Product $27-\mathrm{Cl}$ & 356.56150 & 0.00796 & 0.06926 & -0.15061 & -1333.14282 & -1334.15904 & -1333.86226 & -1334.31579 & -1334.35628 \\
\hline Product28-Cl & 401.89850 & 0.01058 & 0.12476 & -0.20709 & -1411.21556 & -1412.60970 & -1412.23768 & -1412.79447 & -1412.84011 \\
\hline Product-Product1 & 364.87510 & 0.00916 & 0.18821 & -0.17885 & -235.36702 & -236.48755 & -236.26151 & -236.57310 & -236.61454 \\
\hline
\end{tabular}




\begin{tabular}{|c|c|c|c|c|c|c|c|c|c|}
\hline Product-Product2 & 432.46010 & 0.01336 & 0.30083 & -0.29180 & \begin{tabular}{|l|}
-391.47619 \\
\end{tabular} & -393.36143 & -392.98232 & -393.50437 & -393.55348 \\
\hline Product-Product3 & 427.12760 & 0.01181 & 0.24475 & -0.23533 & -313.43615 & -314.92262 & -314.62515 & -315.03768 & \begin{tabular}{|l|}
-315.08618 \\
\end{tabular} \\
\hline Product-Product4 & 492.94190 & 0.01613 & 0.35724 & -0.34828 & \begin{tabular}{|l|}
-469.54446 \\
\end{tabular} & -471.79709 & -471.34635 & -471.96941 & -472.02539 \\
\hline Product-Product5 & 358.53010 & 0.00864 & 0.14080 & -0.17885 & \begin{tabular}{|l|}
-306.97434 \\
\end{tabular} & -308.24347 & -307.93571 & -308.35660 & -308.39732 \\
\hline Product-Product6 & 420.92480 & 0.01119 & 0.19721 & -0.23533 & -385.05409 & -386.68854 & -386.31045 & -386.83130 & -386.87910 \\
\hline Product-Product7 & 455.51620 & 0.01380 & 0.25165 & -0.29180 & -463.11083 & -465.13294 & -464.67887 & -465.30495 & -465.35668 \\
\hline Product-Product8 & 531.20880 & 0.01652 & 0.30792 & -0.34828 & -541.19006 & -543.57830 & -543.05390 & -543.77979 & -543.84012 \\
\hline Product-Product9 & 336.77150 & 0.00779 & 0.14230 & -0.17885 & -306.99195 & -308.28070 & -307.95953 & -308.39080 & -308.42904 \\
\hline Product-Product10 & 416.82180 & 0.01249 & 0.25414 & -0.29180 & \begin{tabular}{|l|}
-463.11326 \\
\end{tabular} & -465.16074 & -464.68910 & -465.32862 & -465.37596 \\
\hline Product-Product11 & 403.68200 & 0.01090 & 0.19803 & -0.23533 & -385.04611 & -386.68358 & -386.30348 & -386.82531 & -386.87115 \\
\hline Product-Product12 & 480.23770 & 0.01568 & 0.30971 & -0.34828 & -541.16610 & -543.56295 & -543.03336 & -543.76247 & -543.81701 \\
\hline Product-Product13 & 327.98840 & 0.00741 & 0.09164 & -0.17885 & -378.43343 & -379.88330 & -379.50047 & -380.03179 & -380.06903 \\
\hline Product-Product14 & 408.39060 & 0.01206 & 0.20329 & -0.29180 & -534.57130 & -536.77677 & -536.24656 & -536.98317 & -537.02955 \\
\hline Product-Product15 & 399.39240 & 0.01052 & 0.14753 & -0.23533 & \begin{tabular}{|l|}
-456.49497 \\
\end{tabular} & -458.29597 & -457.85363 & -458.47561 & -458.52097 \\
\hline Product-Product16 & 486.41410 & 0.01580 & 0.25812 & -0.34828 & -612.63255 & -615.18913 & -614.59931 & -615.42744 & -615.48267 \\
\hline Product-Product17 & 369.39910 & 0.00909 & 0.13208 & -0.17885 & -952.30851 & -953.47677 & -953.18244 & -953.61894 & -953.66089 \\
\hline Product-Product18 & 448.62640 & 0.01374 & 0.24393 & -0.29180 & -1108.42373 & -1110.35456 & -1109.90794 & -1110.55412 & -1110.60507 \\
\hline Product-Product19 & 445.21190 & 0.01203 & 0.19106 & -0.23533 & -1030.37620 & -1031.91210 & -1031.55012 & -1032.07931 & -1032.12987 \\
\hline Product-Product20 & 514.45320 & 0.01679 & 0.30274 & -0.34828 & -1186.48758 & -1188.78679 & -1188.27263 & -1189.01138 & \begin{tabular}{|l|}
-1189.06980 \\
\end{tabular} \\
\hline Product-Product21 & 393.40320 & 0.00984 & 0.13390 & -0.17885 & -952.30997 & -953.47430 & -953.18566 & -953.61259 & -953.65727 \\
\hline Product-Product22 & 448.48600 & 0.01415 & 0.24626 & -0.29180 & \begin{tabular}{|l|}
-1108.42377 \\
\end{tabular} & -1110.35634 & -1109.91279 & -1110.55067 & \begin{tabular}{|l|}
-1110.60160 \\
\end{tabular} \\
\hline Product-Product23 & 450.49440 & 0.01212 & 0.19127 & -0.23533 & -1030.37796 & -1031.91301 & -1031.55140 & -1032.07953 & -1032.13069 \\
\hline Product-Product24 & 511.99530 & 0.01699 & 0.30295 & -0.34828 & -1186.49152 & -1188.79570 & -1188.27852 & -1189.01811 & -1189.07625 \\
\hline Product-Product 25 & 385.33310 & 0.00933 & 0.07872 & -0.17885 & -1669.26014 & -1670.48040 & -1670.11250 & -1670.67697 & \begin{tabular}{|l|}
-1670.72073 \\
\end{tabular} \\
\hline Product-Product26 & 465.37730 & 0.01452 & 0.18996 & -0.29180 & \begin{tabular}{|l|}
-1825.37950 \\
\end{tabular} & -1827.36563 & -1826.84326 & -1827.61796 & \begin{tabular}{|l|}
-1827.67081 \\
\end{tabular} \\
\hline Product-Product 27 & 464.28190 & 0.01291 & 0.13734 & -0.23533 & \begin{tabular}{|l|}
-1747.33631 \\
\end{tabular} & -1748.92082 & -1748.48634 & -1749.14154 & \begin{tabular}{|l|}
-1749.19426 \\
\end{tabular} \\
\hline Product-Product28 & 534.56920 & 0.01780 & 0.24875 & -0.34828 & -1903.45036 & -1905.80646 & -1905.21557 & -1906.08293 & -1906.14363 \\
\hline
\end{tabular}

${ }^{*} \mathrm{Cl}$ radical has -0.00134 Spin Orbit Coupling 


\section{Table S4. MP2/6-31G(d) Optimized Geometries}

\section{Reactant Radicals}

\section{CH3}

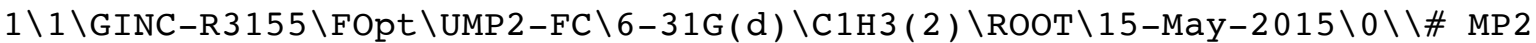
/6-31G(d) SCF=Tight INT(grid=ultrafine) OPT IOP(2/17=4) maxdisk=134217 $728 \backslash \backslash \mathrm{tk} . \mathrm{MP} 2 \backslash \backslash 0,2 \backslash \mathrm{H},-2.703380434,1.7772421219,-0.0577960108 \backslash \mathrm{C},-2.716599$ $0122,1.8395553275,1.0190961766 \backslash \mathrm{H},-2.7036726903,0.9379157158,1.61123859$ $53 \backslash \mathrm{H},-2.7457378636,2.8029068348,1.5035432389 \backslash \backslash$ Version=ES64L-G09RevD.01 $\backslash$ State $=2-\mathrm{A} \backslash \mathrm{HF}=-39.5589087 \backslash \mathrm{MP} 2=-39.6687503 \backslash \mathrm{PUHF}=-39.561992 \backslash \mathrm{PMP} 2-0=-39.6$ $707474 \backslash \mathrm{S} 2=0.761796 \backslash \mathrm{S} 2-1=0.753452 \backslash \mathrm{S} 2 \mathrm{~A}=0.750079 \backslash \mathrm{RMSD}=2.446 \mathrm{e}-09 \backslash \mathrm{RMSF}=4.03$ $0 e-05 \backslash \mathrm{Dipole}=-0.000914,-0.0000481,-0.0000243 \backslash \mathrm{PG}=\mathrm{C} 01 \quad[\mathrm{X}(\mathrm{C} 1 \mathrm{H} 3)] \backslash \backslash \mathrm{a}$

\section{$\mathrm{CH} 3 \mathrm{CH} 2$}

$1 \backslash 1 \backslash G I N C-R 100 \backslash F O p t \backslash U M P 2-F C \backslash 6-31 G(d) \backslash C 2 H 5$ ( 2$) \backslash R O O T \backslash 15-M a y-2015 \backslash 0 \backslash \backslash \#$ MP2/ 6-31G(d) SCF=Tight INT(grid=ultrafine) OPT IOP(2/17=4) maxdisk=1342177 $28 \backslash \backslash t \mathrm{k} . \mathrm{MP} 2 \backslash \backslash 0,2 \backslash \mathrm{H},-2.710313868,1.6832535302,0.0076236441 \backslash \mathrm{C},-2.80489106$ $96,1.8336653192,1.0751622142 \backslash \mathrm{H},-2.908657361,0.9512694625,1.6930758496 \backslash$ C, $-2.4872033337,3.1559196192,1.6867965121 \backslash \mathrm{H},-2.7962821689,3.9795975809$ $, 1.0374686679 \backslash \mathrm{H},-2.9862737661,3.2784360756,2.6519494437 \backslash \mathrm{H},-1.408870689$ , $3.276525435,1.8660729092 \backslash \backslash$ Version=ES64L-G09RevD. $01 \backslash$ State $=2-A^{\prime} \backslash H F=-78$. $5969117 \backslash \mathrm{MP} 2=-78.8355986 \backslash \mathrm{PUHF}=-78.6001833 \backslash \mathrm{PMP} 2-0=-78.8377146 \backslash \mathrm{S} 2=0.76271$ $6 \backslash \mathrm{S} 2-1=0.753687 \backslash \mathrm{S} 2 \mathrm{~A}=0.75011 \backslash \mathrm{RMSD}=4.703 \mathrm{e}-09 \backslash \mathrm{RMSF}=8.261 \mathrm{e}-05 \backslash \mathrm{Dipole}=0.082$ $0566,0.0448778,0.0291466 \backslash \mathrm{PG}=\mathrm{CS} \quad[\mathrm{SG}(\mathrm{C} 2 \mathrm{H} 1), \mathrm{X}(\mathrm{H} 4)] \backslash \backslash @$

\section{$\mathrm{HO}$}

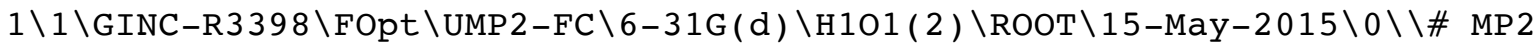
/6-31G(d) SCF=Tight INT(grid=ultrafine) OPT IOP(2/17=4) maxdisk=134217 $728 \backslash \backslash \mathrm{tk} . \mathrm{MP} 2 \backslash \backslash 0,2 \backslash 0,-0.0104556205,0 ., 0 . \backslash \mathrm{H},-0.9895443795,0 ., 0 . \backslash \backslash$ Version= ES64L-G09RevD. $01 \backslash \mathrm{HF}=-75.38186 \backslash \mathrm{MP} 2=-75.5210332 \backslash \mathrm{PUHF}=-75.3845418 \backslash \mathrm{PMP} 2-0=$ $-75.522572 \backslash \mathrm{S} 2=0.755478 \backslash \mathrm{S} 2-1=0.7508 \backslash \mathrm{S} 2 \mathrm{~A}=0.750016 \backslash \mathrm{RMSD}=2.434 \mathrm{e}-09 \backslash \mathrm{RMSF}=1$. $874 \mathrm{e}-07 \backslash \mathrm{Dipole}=-0.7322247,0 ., 0 . \backslash \mathrm{PG}=\mathrm{C} * \mathrm{~V} \quad[\mathrm{C} *(\mathrm{H} 101)] \backslash \backslash \mathrm{a}$

\section{$\mathrm{CH} 30$}

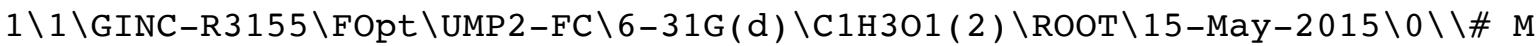
P2/6-31G(d) SCF=Tight INT(grid=ultrafine) OPT IOP(2/17=4) maxdisk=1342 $17728 \backslash \backslash t k$.MP $2 \backslash \backslash 0,2 \backslash 0,-3.265683154,1.9934158231,2.1221914055 \backslash \mathrm{C},-2.66290$ $01455,2.9142105065,1.2751884366 \backslash \mathrm{H},-1.7522101211,3.2303741742,1.8062295$ $354 \backslash \mathrm{H},-2.361384855,2.4674351758,0.3208799333 \backslash \mathrm{H},-3.2858947244,3.8017323$ $204,1.1156006893 \backslash \backslash$ Version=ES64L-G09RevD. $01 \backslash$ State $=2-A \backslash H F=-114.4203489 \backslash \mathrm{M}$ $\mathrm{P} 2=-114.6860822 \backslash \mathrm{PUHF}=-114.4235357 \backslash \mathrm{PMP} 2-0=-114.6879815 \backslash \mathrm{S} 2=0.757688 \backslash \mathrm{S} 2-1$ $=0.751455 \backslash \mathrm{S} 2 \mathrm{~A}=0.750043 \backslash \mathrm{RMSD}=7.761 \mathrm{e}-09 \backslash \mathrm{RMSF}=2.802 \mathrm{e}-04 \backslash \mathrm{Dipole}=0.3919138$, $0.5282209,-0.4304288 \backslash \mathrm{PG}=\mathrm{C} 01[\mathrm{X}(\mathrm{C} 1 \mathrm{H} 301)] \backslash \backslash @$

\section{HS}

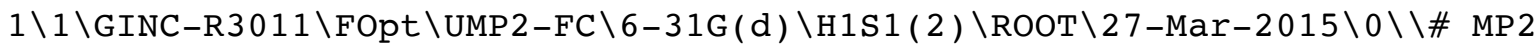
/6-31G(d) SCF=Tight INT(grid=ultrafine) OPT IOP(2/17=4) maxdisk=134217 $728 \backslash \backslash \mathrm{hs} . \mathrm{MP} 2 \backslash \backslash 0,2 \backslash \mathrm{S},-2.3382258831,2.7809142291,-1.1000789475 \backslash \mathrm{H},-2.03731$ $41169,1.4727757709,-1.0243810525 \backslash \backslash$ Version=ES64L-G09RevD $.01 \backslash \mathrm{HF}=-398.064$ $2901 \backslash \mathrm{MP} 2=-398.1619418 \backslash \mathrm{PUHF}=-398.0668019 \backslash \mathrm{PMP} 2-0=-398.1632996 \backslash \mathrm{S} 2=0.75765$ $\backslash \mathrm{S} 2-1=0.750615 \backslash \mathrm{S} 2 \mathrm{~A}=0.75001 \backslash \mathrm{RMSD}=8.713 \mathrm{e}-09 \backslash \mathrm{RMSF}=5.439 \mathrm{e}-08 \backslash \mathrm{Dipole}=0.0976$ $709,-0.4245998,0.0245703 \backslash P G=C * V \quad[C *(H 1 S 1)] \backslash \backslash @$

\section{$\mathrm{CH} 3 \mathrm{~S}$}

$1 \backslash 1 \backslash G I N C-R 2830 \backslash F O p t \backslash U M P 2-F C \backslash 6-31 G(d) \backslash C 1 H 3 S 1(2) \backslash R O O T \backslash 27-M a r-2015 \backslash 0 \backslash \backslash \# M$ P2/6-31G(d) SCF=Tight INT(grid=ultrafine) OPT IOP(2/17=4) maxdisk=1342 
$17728 \backslash \backslash \mathrm{CH} 3 \mathrm{~S} . \mathrm{MP} 2 \backslash \backslash 0,2 \backslash \mathrm{S},-3.3730455322,1.7105140885,-0.0548814219 \backslash \mathrm{C},-1.5$ $799932109,1.8695284665,0.0293391368 \backslash \mathrm{H},-1.1580048545,1.0666298382,-0.58$ $49977323 \backslash \mathrm{H},-1.2422956131,2.8223960925,-0.3806144343 \backslash \mathrm{H},-1.2138807894,1$. $7430315144,1.0491644517 \backslash \backslash$ Version=ES64L-G09RevD .01 \State=2-A \HF=-437.10 $12831 \backslash \mathrm{MP} 2=-437.3308953 \backslash \mathrm{PUHF}=-437.1039015 \backslash \mathrm{PMP} 2-0=-437.3323323 \backslash \mathrm{S} 2=0.7583$ $31 \backslash \mathrm{S} 2-1=0.750802 \backslash \mathrm{S} 2 \mathrm{~A}=0.750021 \backslash \mathrm{RMSD}=5.309 e-09 \backslash \mathrm{RMSF}=1.909 e-04 \backslash \mathrm{Dipole}=0.6$ $787357,0.0350111,0.012985 \backslash P G=C 01 \quad[X(\mathrm{C} 1 \mathrm{H} 3 \mathrm{~S} 1)] \backslash \backslash @$

\section{Reactant Agents}

\section{$\mathrm{CH} 2=\mathrm{CH} 2$}

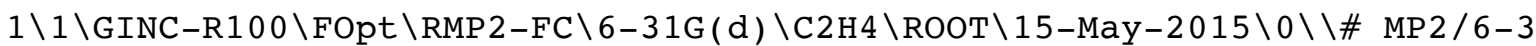
$1 \mathrm{G}(\mathrm{d}) \mathrm{SCF}=\mathrm{Tight}$ INT(grid=ultrafine) OPT IOP(2/17=4) maxdisk=134217728 $\backslash t \mathrm{k} . M P 2 \backslash \backslash 0,1 \backslash \mathrm{C},-5.1392247549,1.8156961972,0.9944611236 \backslash \mathrm{C},-5.5699856804$ , $0.6821898238,0.4327894484 \backslash \mathrm{H},-5.114333505,2.7508326994,0.4446301944 \backslash \mathrm{H}$, $-4.7965762379,1.8477056068,2.0235292917 \backslash \mathrm{H},-5.5948769303,-0.2529466785$, $0.9826203777 \backslash \mathrm{H},-5.9126341974,0.6501804141,-0.5962787196 \backslash \backslash$ Version=ES $64 \mathrm{~L}$ -G09RevD.01 $\backslash \mathrm{HF}=-78.0309821 \backslash \mathrm{MP} 2=-78.2850278 \backslash \mathrm{RMSD}=1.846 \mathrm{e}-09 \backslash \mathrm{RMSF}=1.428 \mathrm{e}-$ $05 \backslash \mathrm{Dipole}=0 ., 0 ., 0 . \backslash \mathrm{PG}=\mathrm{D} 02 \mathrm{H} \quad[\mathrm{C} 2 "(\mathrm{C} 1 . \mathrm{C} 1), \mathrm{SG}(\mathrm{H} 4)] \backslash \backslash \mathrm{Q}$

\section{$\mathrm{CH} 2=\mathrm{C}(\mathrm{CH} 3) 2$}

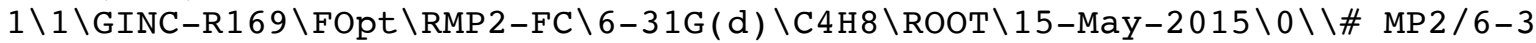
$1 \mathrm{G}(\mathrm{d}) \mathrm{SCF}=\mathrm{Tight}$ INT(grid=ultrafine) OPT IOP(2/17=4) maxdisk=134217728 $\backslash$ tk.MP $2 \backslash \backslash 0,1 \backslash \mathrm{C},-5.1492049957,1.7916995639,0.9805430512 \backslash \mathrm{C},-5.5789339439$ $, 0.6477032803,0.4285623031 \backslash \mathrm{C},-5.1171590723,3.0852012017,0.2135385053 \backslash \mathrm{C}$ $,-4.6695043627,1.8609233453,2.4041440651 \backslash \mathrm{H},-5.5991983879,-0.2827371,0$. $9884482501 \backslash \mathrm{H},-5.9236661201,0.606058248,-0.6003499847 \backslash \mathrm{H},-4.7228330936,0$ $.884963937,2.8933873457 \backslash \mathrm{H},-3.6323035778,2.2127270612,2.4484781313 \backslash \mathrm{H},-5$ $.271558177,2.5704796595,2.983434646 \backslash \mathrm{H},-4.0978948617,3.4863009692,0.170$ $1424226 \backslash \mathrm{H},-5.4794146418,2.9538794573,-0.8092328508 \backslash \mathrm{H},-5.7372927653,3.8$ $439313766,0.7047861152 \backslash \backslash$ Version=ES64L-G09RevD. $01 \backslash$ State $=1-A \backslash H F=-156.109$ $6929 \backslash M P 2=-156.6273418 \backslash \mathrm{RMSD}=8.755 \mathrm{e}-09 \backslash \mathrm{RMSF}=7.135 \mathrm{e}-05 \backslash \mathrm{Dipole}=0.0509707,0$ $.1354518,0.065471 \backslash \mathrm{PG}=\mathrm{C} 01 \quad[\mathrm{X}(\mathrm{C} 4 \mathrm{H} 8)] \backslash \backslash @$

\section{$\mathrm{H} 2 \mathrm{C}=\mathrm{O}$}

$1 \backslash 1 \backslash G I N C-R 100 \backslash F O p t \backslash R M P 2-F C \backslash 6-31 G(d) \backslash C 1 H 201 \backslash R O O T \backslash 15-M a y-2015 \backslash 0 \backslash \backslash \#$ MP2 /6 $-31 \mathrm{G}(\mathrm{d}) \mathrm{SCF}=\mathrm{Tight}$ INT(grid=ultrafine) OPT IOP(2/17=4) maxdisk=13421772 $8 \backslash \backslash t \mathrm{k} . \mathrm{MP} 2 \backslash \backslash 0,1 \backslash \mathrm{C},-5.1395186345,1.8124215241,0.9957527186 \backslash 0,-5.53145661$ $7,0.7714834461,0.4911230087 \backslash \mathrm{H},-5.1145150974,2.7635473223,0.4355597947 \backslash$ $\mathrm{H},-4.7874948676,1.8631077548,2.0410595486 \backslash \backslash$ Version=ES64L-G09RevD.01\St ate $=1-A^{\prime} \backslash H F=-113.8635371 \backslash M P 2=-114.1677473 \backslash R M S D=8.502 e-09 \backslash R M S F=2.046 e-0$ $4 \backslash \mathrm{Dipole}=0.2877936,0.7643246,0.370554 \backslash \mathrm{PG}=\mathrm{CS} \quad[\mathrm{SG}(\mathrm{C} 1 \mathrm{H} 2 \mathrm{O} 1)] \backslash \backslash @$

\section{( $\mathrm{CH} 3$ ) $2 \mathrm{C}=\mathrm{O}$}

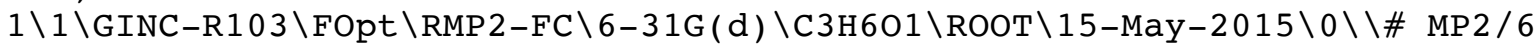
$-31 \mathrm{G}(\mathrm{d}) \mathrm{SCF}=\mathrm{Tight}$ INT(grid=ultrafine) OPT IOP(2/17=4) maxdisk=13421772 $8 \backslash \backslash \mathrm{tk} . M P 2 \backslash \backslash 0,1 \backslash \mathrm{C},-5.1441079754,1.796608757,0.9918241318 \backslash 0,-5.532814232$ $3,0.747608877,0.4863895963 \backslash \mathrm{C},-5.1236898259,3.0951425146,0.2146322166 \backslash \mathrm{C}$ $,-4.6604445229,1.8586087586,2.4246319691 \backslash \mathrm{H},-4.7279899521,0.8674387963$, $2.8737569412 \backslash \mathrm{H},-3.6245424936,2.2112805256,2.4608940837 \backslash \mathrm{H},-5.2646944984$ , $2.5676758589,2.9998052732 \backslash \mathrm{H},-4.1069818722,3.4995430302,0.1760242582 \backslash \mathrm{H}$ $,-5.4887935977,2.9183260049,-0.797361651 \backslash \mathrm{H},-5.7517450296,3.8434998771$, $0.7089081809 \backslash \backslash$ Version=ES64L-G09RevD.01 \State $=1-\mathrm{A} \backslash \mathrm{HF}=-191.9597132 \backslash \mathrm{MP} 2=-$ $192.5239051 \backslash \mathrm{RMSD}=4.157 \mathrm{e}-09 \backslash \mathrm{RMSF}=1.011 \mathrm{e}-05 \backslash \mathrm{Dipole}=0.345471,0.9324295,0$. $4492505 \backslash P G=C 01 \quad[X(C 3 H 601)] \backslash \backslash @$

\section{$\mathrm{CH} 3 \mathrm{CH}=\mathrm{CH} 2$}

$1 \backslash 1 \backslash G I N C-R 92 \backslash F O p t \backslash R M P 2-F C \backslash 6-31 G(d) \backslash C 3 H 6 \backslash R O O T \backslash 15-M a y-2015 \backslash 0 \backslash \backslash \#$ MP $2 / 6-31$ G(d) SCF=Tight INT(grid=ultrafine) OPT IOP(2/17=4) maxdisk=134217728\\ tk.MP $2 \backslash \backslash 0,1 \backslash \mathrm{C},-5.8334480247,2.3715164952,0.4958134479 \backslash \mathrm{C},-5.7138377291$, 
$1.0478459542,0.3402343748 \backslash \mathrm{H},-5.9698910726,3.033238912,-0.355179176 \backslash \mathrm{H},-$ $5.7990669601,2.8360382699,1.4756464849 \backslash \mathrm{H},-5.578504641,0.4229350643,1.2$ $22410795 \backslash \mathrm{C},-5.7520466173,0.3401267242,-0.9808555288 \backslash \mathrm{H},-6.5702099957,-0$ $.3871581261,-1.0150862526 \backslash \mathrm{H},-5.890748069,1.0499257801,-1.8009215989 \backslash \mathrm{H}$, $-4.8233725985,-0.2123653184,-1.1592467262 \backslash \backslash$ Version=ES64L-G0 9RevD.01 \St ate $=1-\mathrm{A}^{\prime} \backslash \mathrm{HF}=-117.0705928 \backslash \mathrm{MP} 2=-117.455545 \backslash \mathrm{RMSD}=7.506 \mathrm{e}-09 \backslash \mathrm{RMSF}=6.172 \mathrm{e}-06$ $\backslash$ Dipole $=0.0063017,-0.0996748,-0.0444942 \backslash P G=C S \quad[S G(C 3 H 4), x(H 2)] \backslash \backslash @$

$\mathrm{H} 2 \mathrm{C}=\mathrm{S}$

$1 \backslash 1 \backslash G I N C-R 3011 \backslash F O p t \backslash R M P 2-F C \backslash 6-31 G(d) \backslash C 1 H 2 S 1 \backslash R O O T \backslash 27-M a r-2015 \backslash 0 \backslash \backslash \#$ MP2 / 6-31G(d) SCF=Tight INT(grid=ultrafine) OPT IOP(2/17=4) maxdisk=1342177 $28 \backslash \backslash t \mathrm{k} . M P 2 \backslash \backslash 0,1 \backslash \mathrm{S}, 0.7457591466,-0.4312116293,-0.0897242226 \backslash \mathrm{C}, 1.4756606$ $459,0.9823487842,0.199400202 \backslash \mathrm{H}, 0.9716593205,1.936884202,0.0441652991 \backslash \mathrm{H}$ $, 2.5025775182,1.0405153782,0.561769433 \backslash \backslash$ Version=ES64L-G09RevD.01 \State $=1-\mathrm{A} 1 \backslash \mathrm{HF}=-436.5058812 \backslash \mathrm{MP} 2=-436.7551126 \backslash \mathrm{RMSD}=1.514 \mathrm{e}-09 \backslash \mathrm{RMSF}=2.019 \mathrm{e}-04 \backslash \mathrm{D}$ ipole $=0.3030685,0.5869362,0.1200498 \backslash \mathrm{PG}=\mathrm{C} 02 \mathrm{~V} \quad[\mathrm{C} 2(\mathrm{C} 1 \mathrm{~S} 1), \mathrm{SGV}(\mathrm{H} 2)] \backslash \backslash @$

( $\mathrm{CH} 3$ ) $2 \mathrm{C}=\mathrm{S}$

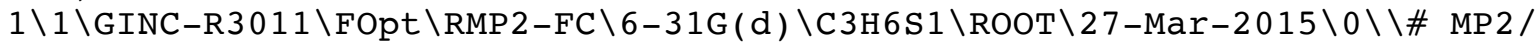
6-31G(d) SCF=Tight INT(grid=ultrafine) OPT IOP(2/17=4) maxdisk=1342177 $28 \backslash \backslash$ diMetk.MP2 $\backslash \backslash 0,1 \backslash \mathrm{C}, 0.6021967741,1.3684102964,-1.1882038449 \backslash \mathrm{S}, 1.3835$ $523659,1.3563324677,0.2404940371 \backslash \mathrm{C}, 1.2982022738,1.1110125514,-2.498262$ $2269 \backslash \mathrm{H}, 0.8427853943,0.2486111855,-2.999383979 \backslash \mathrm{H}, 2.362313084,0.92314004$ $13,-2.3534523086 \backslash \mathrm{H}, 1.1695288815,1.9722773096,-3.1645358035 \backslash \mathrm{C},-0.874473$ $9782,1.6378577412,-1.3055690031 \backslash \mathrm{H},-1.0445574918,2.5098002929,-1.948257$ $2182 \backslash \mathrm{H},-1.3253272706,1.8164182446,-0.3291238831 \backslash \mathrm{H},-1.3721350327,0.7861$ $738692,-1.7843437698 \backslash \backslash$ Version $=\mathrm{ES} 64 \mathrm{~L}-\mathrm{G} 0$ 9RevD.01 $\backslash$ State $=1-\mathrm{A} \backslash \mathrm{HF}=-514.59348$ $83 \backslash M P 2=-515.1058793 \backslash \mathrm{RMSD}=4.235 \mathrm{e}-09 \backslash \mathrm{RMSF}=1.476 \mathrm{e}-04 \backslash \mathrm{Dipole}=-0.4922843,0$. $0075895,-0.9001263 \backslash P G=C 01 \quad[\mathrm{X}(\mathrm{C} 3 \mathrm{H} 6 \mathrm{~S} 1)] \backslash \backslash @$

\section{Transition_States}

1-) $\mathrm{CH} 3+\mathrm{CH} 2=\mathrm{CH} 2 \rightarrow \mathrm{CH} 3 \mathrm{CH} 2 \mathrm{CH} 2$

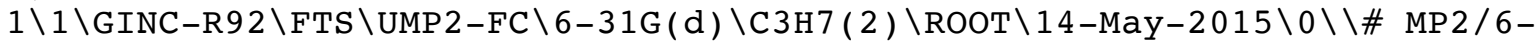
$31 \mathrm{G}(\mathrm{d}) \mathrm{SCF}=\mathrm{Tight}$ INT (grid=ultrafine) OPT=(TS, calcfc, noeigentest, maxcyc $=200) \operatorname{IOP}(2 / 17=4)$ maxdisk=134217728 \HS_diMetk.MP $2 \backslash \backslash 0,2 \backslash \mathrm{H},-2.650211138$ $9,1.7780571765,-0.039746108 \backslash \mathrm{C},-2.8757935412,1.8359888185,1.0172948922 \backslash$ $\mathrm{H},-2.6505008524,0.952826756,1.6011372356 \backslash \mathrm{H},-2.6928839383,2.7907474007$, $1.4973957129 \backslash \mathrm{C},-5.1370454285,1.8115186269,1.0039406116 \backslash \mathrm{C},-5.5721774581$ $, 0.67668152,0.4297591234 \backslash \mathrm{H},-5.1164676322,2.7402496418,0.4451721703 \backslash \mathrm{H},-$ $5.1168651776,1.9116730287,2.0831747938 \backslash \mathrm{H},-5.7178064825,-0.2313113359,1$ $.003802427 \backslash \mathrm{H},-5.7175343505,0.6012193666,-0.6418568589 \backslash \backslash$ Version=ES64L-G 09 RevD.01 $\backslash$ State $=2-A \backslash H F=-117.5743951 \backslash \mathrm{MP} 2=-117.9312438 \backslash \mathrm{PUHF}=-117.5921836$ $\backslash \mathrm{PMP} 2-0=-117.9465766 \backslash \mathrm{S} 2=0.990304 \backslash \mathrm{S} 2-1=0.922508 \backslash \mathrm{S} 2 \mathrm{~A}=0.758809 \backslash \mathrm{RMSD}=5.917$ $e-09 \backslash \mathrm{RMSF}=1.679 \mathrm{e}-05 \backslash \mathrm{Dipole}=0.0771801,0.0679096,0.0343597 \backslash \mathrm{PG}=\mathrm{C} 01 \quad[\mathrm{X}(\mathrm{C} 3 \mathrm{H}$ 7 ) $] \backslash \backslash @$

$2-) \mathrm{CH} 3+\mathrm{CH} 2=\mathrm{C}(\mathrm{CH} 3) 2->\mathrm{CH} 3 \mathrm{CH} 2 \mathrm{C}(\mathrm{CH} 3) 2$

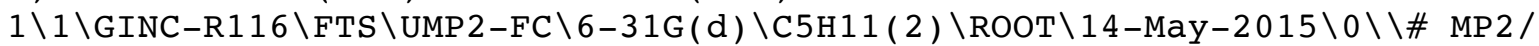
6-31G(d) SCF=Tight INT (grid=ultrafine) OPT=(TS, calcfC, noeigentest, $\operatorname{maxC}$ $\mathrm{yc}=200)$ IOP $(2 / 17=4)$ maxdisk $=134217728 \backslash \backslash \mathrm{HS}$ diMetk.MP $2 \backslash \backslash 0,2 \backslash \mathrm{H},-2.7061656$ $716,1.9821898835,0.0630872532 \backslash \mathrm{C},-2.9686700542,2.0220649356,1.113123624$ $3 \backslash \mathrm{H},-2.7049387924,1.15188123,1.7015955303 \backslash \mathrm{H},-2.8443260987,2.9830021165$ $, 1.5999543201 \backslash \mathrm{C},-5.2166301281,1.8761238448,1.040812691 \backslash \mathrm{C},-5.5580843037$ $, 0.7148736462,0.4532232109 \backslash \mathrm{H},-5.2479113776,2.8052305911,0.4810051173 \backslash \mathrm{H}$ $,-5.2467176666,1.975043019,2.1210485293 \backslash \mathrm{C},-5.6837055006,-0.5600967875$, $1.2338353311 \backslash \mathrm{C},-5.6854120867,0.5890716951,-1.0362931544 \backslash \mathrm{H},-6.644289415$ $4,-1.0499500329,1.0339633795 \backslash \mathrm{H},-4.9002150421,-1.2741353495,0.947629649$ $9 \backslash \mathrm{H},-5.6060182094,-0.3820636794,2.3094506877 \backslash \mathrm{H},-6.64619032,0.137702316$ 
$9,-1.3116107513 \backslash \mathrm{H},-5.6085733819,1.5613322935,-1.5297330529 \backslash \mathrm{H},-4.902211$ $951,-0.0641137228,-1.4431913659 \backslash \backslash$ Version=ES64L-G09RevD.01 \State $=2-\mathrm{A} \backslash \mathrm{HF}$ $=-195.6512592 \backslash M P 2=-196.2739358 \backslash P U H F=-195.6686293 \backslash P M P 2-0=-196.2888317 \backslash \mathrm{S}$ $2=0.978159 \backslash \mathrm{S} 2-1=0.911716 \backslash \mathrm{S} 2 \mathrm{~A}=0.758542 \backslash \mathrm{RMSD}=6.651 \mathrm{e}-09 \backslash \mathrm{RMSF}=4.575 \mathrm{e}-06 \backslash \mathrm{Di}$ pole $=-0.0513164,-0.0853777,-0.0431868 \backslash \mathrm{PG}=\mathrm{C} 01[\mathrm{X}(\mathrm{C} 5 \mathrm{H} 11)] \backslash \backslash @$

\section{$3-) \mathrm{CH} 3 \mathrm{CH} 2+\mathrm{CH} 2=\mathrm{CH} 2->\mathrm{CH} 3 \mathrm{CH} 2 \mathrm{CH} 2 \mathrm{CH} 2$}

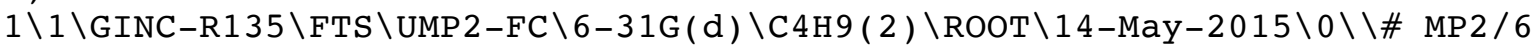
$-31 \mathrm{G}(\mathrm{d}) \mathrm{SCF}=\mathrm{Tight}$ INT(grid=ultrafine) OPT=(TS, calcfC, noeigentest, maxcy

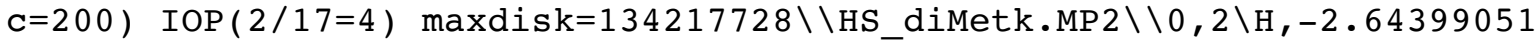
$11,1.6375306425,0.0577309623 \backslash \mathrm{C},-2.9232648656,1.831339445,1.0888112296 \backslash$ $\mathrm{H},-2.900823865,0.9521473329,1.7278808414 \backslash \mathrm{C},-2.4836548354,3.1324265645$, $1.6822954213 \backslash \mathrm{C},-5.1572019065,1.9968363624,0.8413171716 \backslash \mathrm{C},-5.4606527137$ , 3.089893748,0.1206779248\H, $-5.1524680172,1.0146639788,0.3815651713 \backslash \mathrm{H}$, $-5.2441861057,2.0092892,1.9224969844 \backslash \mathrm{H},-5.5057047719,3.062749099,-0.96$ $23596726 \backslash \mathrm{H},-5.5992127213,4.0586905098,0.5870572165 \backslash \mathrm{H},-2.7580573622,3.9$ $672173746,1.0318956687 \backslash \mathrm{H},-2.9455683797,3.3000051642,2.65992078 \backslash \mathrm{H},-1.39$ $50709447,3.1679375782,1.8256903008 \backslash \backslash$ Version=ES64L-G09RevD.01 \State=2-A $\backslash \mathrm{HF}=-156.6114576 \backslash \mathrm{MP} 2=-157.0999724 \backslash \mathrm{PUHF}=-156.629083 \backslash \mathrm{PMP} 2-0=-157.1151448$ $\backslash \mathrm{S} 2=0.986164 \backslash \mathrm{S} 2-1=0.918968 \backslash \mathrm{S} 2 \mathrm{~A}=0.758843 \backslash \mathrm{RMSD}=8.087 \mathrm{e}-09 \backslash \mathrm{RMSF}=7.535 \mathrm{e}-06 \backslash$ Dipole $=0.1507549,-0.0665258,0.0645258 \backslash P G=C 01 \quad[X(C 4 H 9)] \backslash \backslash @$

\section{$4-) \mathrm{CH} 3 \mathrm{CH} 2+\mathrm{CH} 2=\mathrm{C}(\mathrm{CH} 3) 2->\mathrm{CH} 3 \mathrm{CH} 2 \mathrm{CH} 2 \mathrm{C}(\mathrm{CH} 3) 2$}

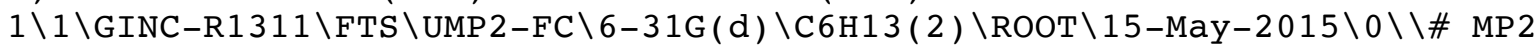
/6-31G(d) SCF=Tight INT (grid=ultrafine) OPT=(TS, calcfC, noeigentest, $\max$ cyc $=200)$ IOP $(2 / 17=4)$ maxdisk $=402653184 \backslash \backslash$ HS_diMetk.MP $2 \backslash \backslash 0,2 \backslash H,-2.725450$ $74,1.4711763078,0.1412774961 \backslash \mathrm{C},-3.024672038 \overline{8}, 1.7195599291,1.1557034809$ $\backslash \mathrm{H},-3.0792537062,0.8607662786,1.8207107407 \backslash \mathrm{C},-2.4914470736,2.996609957$ $2,1.7265253722 \backslash \mathrm{C},-5.2233615672,2.0302870074,0.8446317306 \backslash \mathrm{C},-5.44240340$ $48,3.1364768227,0.1093293741 \backslash \mathrm{H},-5.2623465944,1.0483726894,0.3834828218$ $\backslash \mathrm{H},-5.3690889388,2.0518430942,1.9205339421 \backslash \mathrm{C},-5.4169803995,3.105021979$ $6,-1.3906788937 \backslash \mathrm{C},-5.615279067,4.4898966958,0.7342322918 \backslash \mathrm{H},-2.64927076$ $49,3.8295684551,1.0358713022 \backslash \mathrm{H},-2.9842242004,3.2460266633,2.6713295526$ $\backslash \mathrm{H},-1.4125112111,2.938753366,1.9284338397 \backslash \mathrm{H},-6.5602994811,4.947495623$, $0.4152403182 \backslash \mathrm{H},-5.6140970336,4.4317438571,1.825674379 \backslash \mathrm{H},-4.8155613255$, $5.1764360056,0.4274670941 \backslash \mathrm{H},-6.3280231209,3.5504349714,-1.8088312276 \backslash \mathrm{H}$ $,-4.573389749,3.6884162491,-1.7829883651 \backslash \mathrm{H},-5.3292235831,2.0833820476$,

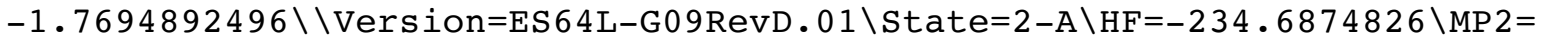
$-235.4424084 \backslash \mathrm{PUHF}=-234.7048665 \backslash \mathrm{PMP} 2-0=-235.4573017 \backslash \mathrm{S} 2=0.976589 \backslash \mathrm{S} 2-1=0$. $91018 \backslash \mathrm{S} 2 \mathrm{~A}=0.758729 \backslash \mathrm{RMSD}=8.735 \mathrm{e}-09 \backslash \mathrm{RMSF}=6.162 \mathrm{e}-06 \backslash \mathrm{Dipole}=0.0250977,0.06$ $19872,-0.0328746 \backslash \mathrm{PG}=\mathrm{C} 01 \quad[\mathrm{X}(\mathrm{C} 6 \mathrm{H} 13)] \backslash \backslash @$

$5-) \mathrm{CH} 3+\mathrm{H} 2 \mathrm{C}=\mathrm{O} \rightarrow \mathrm{CH} 3 \mathrm{OCH} 2$

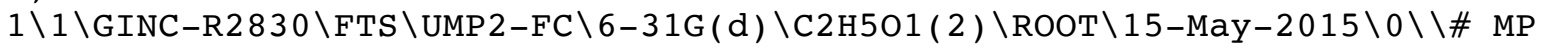
2/6-31G(d) SCF=Tight INT(grid=ultrafine) OPT=(TS, calcfC, noeigentest, ma $\mathrm{xcyc}=200) \operatorname{IOP}(2 / 17=4)$ maxdisk $=134217728 \backslash \backslash$ HS_diMetk.MP $2 \backslash \backslash 0,2 \backslash \mathrm{H},-2.66850$ $31022,1.6782569911,-0.0569494327 \backslash \mathrm{C},-2.9275816294,1.74400072,0.99215900$ $29 \backslash \mathrm{H},-2.7106780838,0.8726164027,1.5969024646 \backslash \mathrm{H},-2.7751650652,2.7044613$ $282,1.4639147971 \backslash 0,-4.8434129137,1.7498835617,0.9461689761 \backslash \mathrm{C},-5.450894$ $665,0.7878254381,0.4620303386 \backslash \mathrm{H},-5.7205634298,-0.0886999361,1.06721994$ $67 \backslash \mathrm{H},-5.6777556885,0.7290278406,-0.6114450243 \backslash \backslash$ Version=ES64L-G0 9RevD. 0 $1 \backslash$ State $=2-A^{\prime} \backslash \mathrm{HF}=-153.3852161 \backslash \mathrm{MP} 2=-153.789065 \backslash \mathrm{PUHF}=-153.4053728 \backslash \mathrm{PMP} 2-0=$ $-153.8064446 \backslash \mathrm{S} 2=0.987793 \backslash \mathrm{S} 2-1=0.921045 \backslash \mathrm{S} 2 \mathrm{~A}=0.757472 \backslash \mathrm{RMSD}=9.490 \mathrm{e}-09 \backslash \mathrm{RMS}$ $\mathrm{F}=3.258 \mathrm{e}-05 \backslash \mathrm{Dipole}=0.2185053,-0.5308103,-0.2530014 \backslash \mathrm{PG}=\mathrm{CS} \quad[\mathrm{SG}(\mathrm{C} 2 \mathrm{H} 1 \mathrm{O} 1), \mathrm{X}$ ( $\mathrm{H} 4$ ) $] \backslash \backslash @$

\section{6-) $\mathrm{CH} 3 \mathrm{CH} 2+\mathrm{H} 2 \mathrm{C}=\mathrm{O}->\mathrm{CH} 3 \mathrm{CH} 2 \mathrm{OCH} 2$}

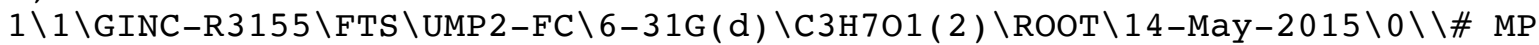
2/6-31G(d) SCF=Tight INT(grid=ultrafine) OPT=(TS, calcfC, noeigentest, ma

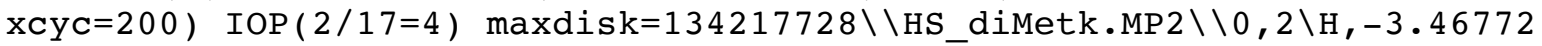


$20025,2.2385913191,-0.1306538791 \backslash \mathrm{C},-3.0405702899,1.9466811845,0.821805$ $1656 \backslash \mathrm{H},-2.450668575,1.0403232571,0.7969127128 \backslash \mathrm{C},-2.6793018324,3.023602$ $517,1.7777368419 \backslash 0,-4.6221685648,1.1367272263,1.5929025251 \backslash \mathrm{C},-5.345655$ $2079,1.8229581627,2.3204152209 \backslash \mathrm{H},-6.1529874339,2.4500413044,1.91327069$ $31 \backslash \mathrm{H},-5.1971474999,1.8736969105,3.4086090364 \backslash \mathrm{H},-2.0686949069,3.8039603$ $844,1.3096472943 \backslash \mathrm{H},-3.5933539147,3.5158733424,2.1481239478 \backslash \mathrm{H},-2.137720$ $7722,2.6318663916,2.6411644413 \backslash \backslash$ Version=ES64L-G09RevD.01 $\backslash$ State=2-A $\backslash H F=$ $-192.4239253 \backslash M P 2=-192.9629521 \backslash \mathrm{PUHF}=-192.4427478 \backslash \mathrm{PMP} 2-0=-192.9790799 \backslash \mathrm{S} 2$ $=0.966817 \backslash \mathrm{S} 2-1=0.903583 \backslash \mathrm{S} 2 \mathrm{~A}=0.756783 \backslash \mathrm{RMSD}=3.930 \mathrm{e}-09 \backslash \mathrm{RMSF}=7.207 \mathrm{e}-06 \backslash \mathrm{Dip}$ $\mathrm{ole}=0.1794499,0.6437355,0.1425806 \backslash \mathrm{PG}=\mathrm{C} 01[\mathrm{X}(\mathrm{C} 3 \mathrm{H} 7 \mathrm{O} 1)] \backslash \backslash \mathrm{a}$

$7-) \mathrm{CH} 3+(\mathrm{CH} 3) 2 \mathrm{C}=\mathrm{O}->\mathrm{CH} 3 \mathrm{OC}(\mathrm{CH} 3) 2$

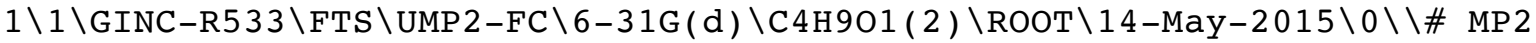
/6-31G(d) SCF=Tight INT(grid=ultrafine) OPT=(TS, calcfc, noeigentest, $\max$

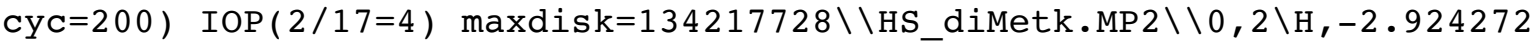
$7708,2.9053456602,1.1934672592 \backslash \mathrm{C},-3.061279 \overline{1} 194,1.8684778256,0.91951784$ $35 \backslash \mathrm{H},-2.6998458868,1.590903565,-0.0635479741 \backslash \mathrm{H},-2.8863409519,1.1472277$ $106,1.7090085163 \backslash 0,-4.9246942451,1.8741054425,0.7248584577 \backslash \mathrm{C},-5.502469$ $6011,0.8153386028,0.3992672224 \backslash \mathrm{C},-5.8737392894,-0.2026495263,1.4418736$ $837 \backslash \mathrm{C},-5.6112089727,0.4196875791,-1.0473814402 \backslash \mathrm{H},-6.5583583387,-0.0850$ $428449,-1.2584696597 \backslash \mathrm{H},-5.5153372054,1.3048679054,-1.678226662 \backslash \mathrm{H},-4.80$ $48371074,-0.2821394001,-1.3071217307 \backslash \mathrm{H},-6.8185426327,-0.699533851,1.20$ $34109716 \backslash \mathrm{H},-5.101291117,-0.9840382853,1.4967790148 \backslash \mathrm{H},-5.9461907618,0.2$ $807876162,2.4174434976 \backslash \backslash$ Version=ES64L-G09RevD. $01 \backslash$ State=2-A $\backslash H F=-231.472$ $1911 \backslash \mathrm{MP} 2=-232.1433089 \backslash \mathrm{PUHF}=-231.4913715 \backslash \mathrm{PMP} 2-0=-232.1596379 \backslash \mathrm{S} 2=0.96197$ $7 \backslash \mathrm{S} 2-1=0.89778 \backslash \mathrm{S} 2 \mathrm{~A}=0.756617 \backslash \mathrm{RMSD}=2.729 \mathrm{e}-09 \backslash \mathrm{RMSF}=9.015 \mathrm{e}-06 \backslash \mathrm{Dipole}=0.129$ $6513,-0.6970012,-0.1606855 \backslash \mathrm{PG}=\mathrm{C} 01 \quad[\mathrm{X}(\mathrm{C} 4 \mathrm{H} 9 \mathrm{O} 1)] \backslash \backslash @$

8-) $\mathrm{CH} 3 \mathrm{CH} 2+(\mathrm{CH} 3) 2 \mathrm{C}=\mathrm{O}->\mathrm{CH} 3 \mathrm{CH} 2 \mathrm{OC}(\mathrm{CH} 3) 2$

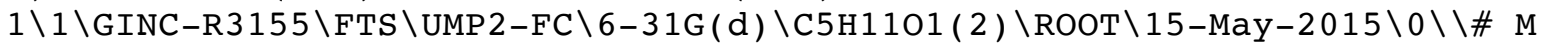
P2/6-31G(d) SCF=Tight INT(grid=ultrafine) OPT=(TS, calcfc, noeigentest, $\mathrm{m}$ axcyc $=200)$ IOP $(2 / 17=4)$ maxdisk=536870912 \\HS_diMetk.MP $2 \backslash \backslash 0,2 \backslash \mathrm{H},-2.7294$ $276268,1.7254702721,-0.0217730388 \backslash \mathrm{C},-2.99778 \overline{8} 2597,1.8114317109,1.02713$ $09009 \backslash \mathrm{H},-2.7725806275,0.9275902208,1.6167744084 \backslash \mathrm{C},-2.8077444431,3.1386$ $938971,1.6784003946 \backslash 0,-4.888082397,1.764309915,0.9547948517 \backslash \mathrm{C},-5.47736$ $82953,0.7830919305,0.458190748 \backslash \mathrm{C},-5.7600231336,-0.4391674711,1.2881697$ $091 \backslash \mathrm{C},-5.6998765643,0.6961932592,-1.0269308939 \backslash \mathrm{H},-3.253935569,3.925963$ $8962,1.0663493791 \backslash \mathrm{H},-3.2945706094,3.1516553212,2.6563126459 \backslash \mathrm{H},-1.74590$ $09652,3.3780092743,1.8220885379 \backslash \mathrm{H},-6.660809496,0.2311255898,-1.2661105$ $741 \backslash \mathrm{H},-5.6546051456,1.6956798194,-1.4627472863 \backslash \mathrm{H},-4.9167760408,0.07824$ $62157,-1.4909242062 \backslash \mathrm{H},-6.7187021053,-0.8954284704,1.0243307918 \backslash \mathrm{H},-4.98$ $23949928,-1.1988190775,1.118963496 \backslash \mathrm{H},-5.7569107286,-0.1717333033,2.346$ $206136 \backslash \backslash$ Version=ES64L-G09RevD.01 \State $=2-A \backslash H F=-270.5125863 \backslash M P 2=-271.31$ $53181 \backslash \mathrm{PUHF}=-270.5315716 \backslash \mathrm{PMP} 2-0=-271.3314797 \backslash \mathrm{S} 2=0.960553 \backslash \mathrm{S} 2-1=0.896731 \backslash$ $\mathrm{S} 2 \mathrm{~A}=0.756726 \backslash \mathrm{RMSD}=9.843 \mathrm{e}-09 \backslash \mathrm{RMSF}=8.051 \mathrm{e}-06 \backslash \mathrm{Dipole}=0.1647385,-0.5623794$ ,$-0.2715897 \backslash \mathrm{PG}=\mathrm{C} 01 \quad[\mathrm{X}(\mathrm{C} 5 \mathrm{H} 1101)] \backslash \backslash @$

9-) $\mathrm{HO}+\mathrm{CH} 2=\mathrm{CH} 2->\mathrm{HOCH} 2 \mathrm{CH} 2$

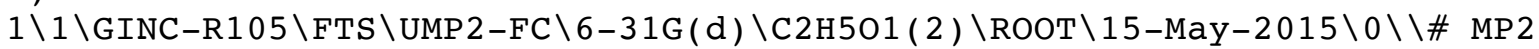
/6-31G(d) SCF=Tight INT(grid=ultrafine) OPT=(TS, calcfc, noeigentest, max

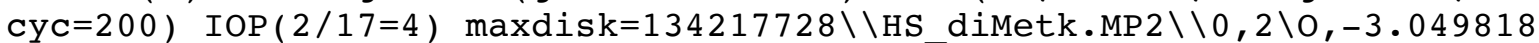
$6385,2.3165418026,1.2178375855 \backslash \mathrm{H},-2.697724 \overline{9} 755,1.5104882006,0.78853496$ $76 \backslash \mathrm{C},-5.0673377491,1.935248607,1.1962948177 \backslash \mathrm{C},-5.0793593599,0.84646189$ $64,0.4157529856 \backslash \mathrm{H},-5.2483278828,2.9202508971,0.7866234409 \backslash \mathrm{H},-5.1032695$ $092,1.8520029663,2.2747078988 \backslash \mathrm{H},-4.9977110252,-0.1512906129,0.83211509$ $05 \backslash \mathrm{H},-5.13460586,0.924037243,-0.6639257865 \backslash \backslash$ Version=ES64L-G09RevD.01\S tate $=2-\mathrm{A} \backslash \mathrm{HF}=-153.4000157 \backslash \mathrm{MP} 2=-153.7955554 \backslash \mathrm{PUHF}=-153.4169575 \backslash \mathrm{PMP} 2-0=-15$ $3.8101969 \backslash \mathrm{S} 2=0.964964 \backslash \mathrm{S} 2-1=0.90566 \backslash \mathrm{S} 2 \mathrm{~A}=0.75574 \backslash \mathrm{RMSD}=9.050 \mathrm{e}-09 \backslash \mathrm{RMSF}=8.7$ 56e-07\Dipole $=-0.2955973,-0.5894791,-0.2674697 \backslash \mathrm{PG}=\mathrm{C} 01[\mathrm{X}(\mathrm{C} 2 \mathrm{H} 501)] \backslash \backslash \mathrm{Q}$ 
$10-) \mathrm{HO}+\mathrm{CH} 2=\mathrm{C}(\mathrm{CH} 3) 2 \rightarrow \mathrm{HOCH} 2 \mathrm{C}(\mathrm{CH} 3) 2$

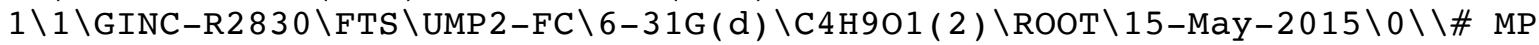
$2 / 6-31 \mathrm{G}(\mathrm{d}) \mathrm{SCF}=\mathrm{Tight}$ INT(grid=ultrafine) $\mathrm{OPT}=(\mathrm{TS}$, calcfC, noeigentest, $\mathrm{ma}$ $\mathrm{xCyc}=200)$ IOP $(2 / 17=4)$ maxdisk $=134217728 \backslash \backslash$ HS diMetk.MP $2 \backslash \backslash 0,2 \backslash 0,-3.26776$ $12109,2.3240176186,0.9170597737 \backslash \mathrm{H},-2.9602850545,1.4807991349,1.3042681$ $018 \backslash \mathrm{C},-5.3014868072,1.9965476395,1.0371777099 \backslash \mathrm{C},-5.3049179641,0.785777$ $6045,0.4689474263 \backslash \mathrm{H},-5.4375892925,2.8944180783,0.4475908309 \backslash \mathrm{H},-5.38880$ $54367,2.1139111221,2.1117250082 \backslash C,-5.2675250911,-0.4775704209,1.280394$ $49 \backslash \mathrm{C},-5.279197724,0.6152266843,-1.0206262954 \backslash \mathrm{H},-6.1306237462,0.0109225$ $069,-1.3545290119 \backslash \mathrm{H},-5.3073082092,1.5791128941,-1.531862633 \backslash \mathrm{H},-4.36952$ $16677,0.0893582992,-1.3309060031 \backslash \mathrm{H},-6.1393724063,-1.1016970949,1.05260$ $5772 \backslash \mathrm{H},-4.3820504369,-1.0763195556,1.0362721754 \backslash \mathrm{H},-5.2645319529,-0.269$ $9315111,2.3532226554 \backslash \backslash$ Version=ES64L-G0 9RevD.01 \State $=2-A \backslash H F=-231.47634$ $34 \backslash M P 2=-232.1393862 \backslash P U H F=-231.4921295 \backslash P M P 2-0=-232.1529709 \backslash S 2=0.944724 \backslash$ $\mathrm{S} 2-1=0.889566 \backslash \mathrm{S} 2 \mathrm{~A}=0.755117 \backslash \mathrm{RMSD}=6.998 \mathrm{e}-09 \backslash \mathrm{RMSF}=6.464 \mathrm{e}-06 \backslash \mathrm{Dipole}=-0.425$ $9147,-0.8543183,0.1272737 \backslash \mathrm{PG}=\mathrm{C} 01[\mathrm{X}(\mathrm{C} 4 \mathrm{H} 9 \mathrm{O} 1)] \backslash \backslash @$

11-) $\mathrm{CH} 3 \mathrm{O}+\mathrm{CH} 2=\mathrm{CH} 2->\mathrm{CH} 3 \mathrm{OCH} 2 \mathrm{CH} 2$

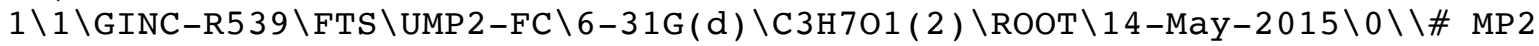
/6-31G(d) SCF=Tight INT(grid=ultrafine) OPT=(TS, calcfC, noeigentest, $\max$ cyc $=200)$ IOP $(2 / 17=4)$ maxdisk $=134217728 \backslash \backslash$ HS_diMetk.MP $2 \backslash \backslash 0,2 \backslash 0,-3.269898$ $8328,1.9838148346,2.1371150419 \backslash \mathrm{C},-2.662077 \overline{9} 629,2.9102401879,1.27864049$ $02 \backslash \mathrm{C},-5.1621059137,1.6078305879,1.5863464796 \backslash \mathrm{C},-5.0139926432,1.1356600$ $775,0.3377125097 \backslash \mathrm{H},-5.4823135073,2.6283704196,1.7606364857 \backslash \mathrm{H},-5.248231$ $3272,0.9348509641,2.4291584911 \backslash \mathrm{H},-4.7946251306,0.0910555445,0.15219028$ $16 \backslash \mathrm{H},-5.030650599,1.7923525258,-0.5244481485 \backslash \mathrm{H},-1.7528101975,3.2297608$ $845,1.8061288126 \backslash \mathrm{H},-2.3630749213,2.4816301555,0.3149204117 \backslash \mathrm{H},-3.280210$ $9647,3.8017218181,1.1032861444 \backslash \backslash$ Version=ES64L-G09RevD.01 $\backslash$ State $=2-A \backslash H F=$ $-192.4283609 \backslash M P 2=-192.9528367 \backslash P U H F=-192.4467517 \backslash$ PMP $2-0=-192.968763 \backslash \mathrm{S} 2=$ $0.984637 \backslash \mathrm{S} 2-1=0.92061 \backslash \mathrm{S} 2 \mathrm{~A}=0.757151 \backslash \mathrm{RMSD}=6.797 \mathrm{e}-09 \backslash \mathrm{RMSF}=1.826 \mathrm{e}-05 \backslash \mathrm{Dipol}$ $\mathrm{e}=-0.1804371,0.2623358,-0.4636279 \backslash \mathrm{PG}=\mathrm{C} 01 \quad[\mathrm{X}(\mathrm{C} 3 \mathrm{H} 7 \mathrm{O} 1)] \backslash \backslash @$

12-) $\mathrm{CH} 3 \mathrm{O}+\mathrm{CH} 2=\mathrm{C}(\mathrm{CH} 3) 2->\mathrm{CH} 3 \mathrm{OCH} 2 \mathrm{C}(\mathrm{CH} 3) 2$

$1 \backslash 1 \backslash$ GINC-R5 42 $\backslash F T S \backslash U M P 2-F C \backslash 6-31 G(d) \backslash C 5 H 1101(2) \backslash R O O T \backslash 14-M a y-2015 \backslash 0 \backslash \backslash \#$ MP $2 / 6-31 \mathrm{G}(\mathrm{d}) \mathrm{SCF}=\mathrm{Tight}$ INT (grid=ultrafine) OPT=(TS, calcfC, noeigentest, ma $\mathrm{xcyc}=200) \operatorname{IOP}(2 / 17=4)$ maxdisk $=402653184 \backslash \backslash$ HS_diMetk.MP $2 \backslash \backslash 0,2 \backslash 0,-3.65731$ $15731,3.0152134673,0.6161460085 \backslash C,-2.8331018502,2.3732616759,1.5450723$ $938 \backslash \mathrm{C},-5.5331544157,2.2807900034,0.6723480038 \backslash \mathrm{C},-5.3267325519,0.976045$ $2227,0.4338274728 \backslash \mathrm{H},-5.7663285688,2.9643188807,-0.1343763164 \backslash \mathrm{H},-5.7543$ $339515,2.6334362704,1.6744043951 \backslash \mathrm{C},-5.1817261144,-0.0242295514,1.54042$ $90674 \backslash \mathrm{C},-5.1165225463,0.4591928977,-0.9570562727 \backslash \mathrm{H},-5.802164158,-0.368$ $2690618,-1.1736464695 \backslash \mathrm{H},-5.2632567298,1.2437773552,-1.7018867183 \backslash \mathrm{H},-4$. $097704454,0.0686326753,-1.0674187143 \backslash \mathrm{H},-5.8993185176,-0.8433757923,1.4$ $123114813 \backslash \mathrm{H},-4.1818243243,-0.4755872543,1.5299972817 \backslash \mathrm{H},-5.3464198027,0$ $.4290768821,2.5205704592 \backslash \mathrm{H},-1.9155674124,2.9777255522,1.5890791656 \backslash \mathrm{H},-$ $3.2619908099,2.3430668056,2.5571660862 \backslash \mathrm{H},-2.5453562193,1.3550439712,1$. $2481206758 \backslash \backslash$ Version=ES6 4L-G09RevD.01 \State $=2-A \backslash H F=-270.5055625 \backslash M P 2=-27$ $1.2985207 \backslash \mathrm{PUHF}=-270.5226686 \backslash \mathrm{PMP} 2-0=-271.3132461 \backslash \mathrm{S} 2=0.960811 \backslash \mathrm{S} 2-1=0.901$ $082 \backslash \mathrm{S} 2 \mathrm{~A}=0.75633 \backslash \mathrm{RMSD}=7.813 \mathrm{e}-09 \backslash \mathrm{RMSF}=3.589 \mathrm{e}-06 \backslash \mathrm{Dipole}=-0.1729965,-0.671$ $9813,0.2805949 \backslash \mathrm{PG}=\mathrm{C} 01 \quad[\mathrm{X}(\mathrm{C} 5 \mathrm{H} 1101)] \backslash \backslash @$

13-) $\mathrm{HO}+\mathrm{H} 2 \mathrm{C}=\mathrm{O}->\mathrm{HOOCH} 2$

$1 \backslash 1 \backslash G I N C-R 143 \backslash F T S \backslash U M P 2-F C \backslash 6-31 G(d) \backslash C 1 H 3 O 2(2) \backslash R O O T \backslash 18-M a y-2015 \backslash 0 \backslash \backslash \#$ MP2 $16-31 \mathrm{G}(\mathrm{d}) \mathrm{SCF}=\mathrm{Tight}$ INT (grid=ultrafine) OPT=(TS, calcfc, noeigentest, max cyc $=200)$ IOP $(2 / 17=4)$ maxdisk $=134217728 \backslash \backslash$ HS_diMetk.MP $2 \backslash \backslash 0,2 \backslash 0,-3.150814$ $9533,1.9491228327,1.1217467311 \backslash \mathrm{H},-2.812445425,2.7382646722,1.585191566$ $8 \backslash 0,-4.4829754427,1.8030297854,1.9444426986 \backslash \mathrm{C},-5.4766799029,1.70637442$ $36,1.1492054945 \backslash \mathrm{H},-5.7240035076,2.528392722,0.4792081155 \backslash \mathrm{H},-6.00056776$ $85,0.7562375641,1.0577333935 \backslash \backslash$ Version=ES64L-G0 9RevD.01 \State $=2-A \backslash H F=-1$ $89.1704169 \backslash \mathrm{MP} 2=-189.6140833 \backslash \mathrm{PUHF}=-189.1951785 \backslash \mathrm{PMP} 2-0=-189.6355761 \backslash \mathrm{S} 2=1$ 
$.022652 \backslash \mathrm{S} 2-1=0.949392 \backslash \mathrm{S} 2 \mathrm{~A}=0.758097 \backslash \mathrm{RMSD}=9.951 \mathrm{e}-09 \backslash \mathrm{RMSF}=1.628 \mathrm{e}-06 \backslash \mathrm{Dipol}$ $\mathrm{e}=-0.441565,0.4589626,-0.1556108 \backslash \mathrm{PG}=\mathrm{C} 01 \quad[\mathrm{X}(\mathrm{C} 1 \mathrm{H} 3 \mathrm{O} 2)] \backslash \backslash @$

$14-) \mathrm{HO}+(\mathrm{CH} 3) 2 \mathrm{C}=\mathrm{O} \rightarrow \mathrm{HOOC}(\mathrm{CH} 3) 2$

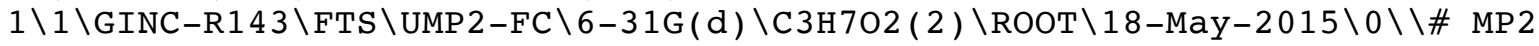
/6-31G(d) SCF=Tight INT(grid=ultrafine) OPT=(TS, calcfC, noeigentest, $\max$ cyc $=200)$ IOP $(2 / 17=4)$ maxdisk=134217728 \HS_diMetk.MP $2 \backslash \backslash 0,2 \backslash 0,-3.265629$ $1751,1.8996664332,1.4215753734 \backslash \mathrm{H},-2.950114 \overline{3} 198,2.7656785695,1.73964196$ $14 \backslash 0,-4.6591624524,1.9254372378,2.0962439257 \backslash \mathrm{C},-5.5806680665,1.7689322$ $588,1.1997700946 \backslash \mathrm{C},-5.8116023366,2.8538708927,0.1987197687 \backslash \mathrm{C},-6.143769$ $1144,0.4006195122,0.9876148687 \backslash \mathrm{H},-6.8479789101,2.8596425532,-0.1485613$ $608 \backslash \mathrm{H},-5.5693457986,3.8245093713,0.6368614674 \backslash \mathrm{H},-5.1647514419,2.701404$ $7622,-0.674766756 \backslash \mathrm{H},-7.18742858,0.4444878014,0.6652085602 \backslash \mathrm{H},-5.5742537$ $723,-0.1186653931,0.2035997317 \backslash \mathrm{H},-6.0643650323,-0.1812189993,1.9071613$ $652 \backslash \backslash$ Version=ES64L-G0 9RevD.01 \State $=2-\mathrm{A} \backslash \mathrm{HF}=-267.2561784 \backslash \mathrm{MP} 2=-267.96785$ $63 \backslash \mathrm{PUHF}=-267.2790321 \backslash \mathrm{PMP} 2-0=-267.987424 \backslash \mathrm{S} 2=0.98342 \backslash \mathrm{S} 2-1=0.915088 \backslash \mathrm{S} 2 \mathrm{~A}=0$ $.757059 \backslash \mathrm{RMSD}=7.655 \mathrm{e}-09 \backslash \mathrm{RMSF}=7.968 \mathrm{e}-06 \backslash \mathrm{Dipole}=-0.4886598,0.420148,-0.45$ $06708 \backslash \mathrm{PG}=\mathrm{C} 01 \quad[\mathrm{X}(\mathrm{C} 3 \mathrm{H} 7 \mathrm{O} 2)] \backslash \backslash @$

$15-) \mathrm{CH} 3 \mathrm{O}+\mathrm{H} 2 \mathrm{C}=\mathrm{O} \rightarrow \mathrm{CH} 30 \mathrm{OCH} 2$

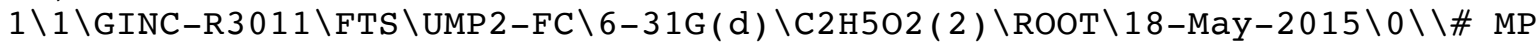
2/6-31G(d) SCF=Tight INT(grid=ultrafine) OPT=(TS, calcfC, noeigentest, ma $\mathrm{xcyc}=200)$ IOP $(2 / 17=4)$ maxdisk $=134217728 \backslash \backslash$ HS_diMetk.MP2 $\backslash \backslash 0,2 \backslash 0,-3.17037$ $9953,1.9098703184,1.1211611533 \backslash C,-2.5430339258,3.0446677215,1.68086135$ $35 \backslash 0,-4.5020311813,1.778840197,1.9487985045 \backslash \mathrm{C},-5.5028194908,1.70408189$ $95,1.1589443232 \backslash \mathrm{H},-5.7598414049,2.5465023672,0.517496359 \backslash \mathrm{H},-6.02621614$ $08,0.7564316115,1.0379963393 \backslash \mathrm{H},-1.5706754394,3.0967348491,1.1808088243$ $\backslash \mathrm{H},-3.1051258369,3.9634146785,1.4811146414 \backslash \mathrm{H},-2.394731627,2.9269553572$ , $2.7588015015 \backslash \backslash$ Version=ES64L-G09RevD. $01 \backslash$ State $=2-A \backslash H F=-228.2030434 \backslash$ MP $2=$ $-228.774512 \backslash \mathrm{PUHF}=-228.2280069 \backslash \mathrm{PMP} 2-0=-228.7962143 \backslash \mathrm{S} 2=1.027344 \backslash \mathrm{S} 2-1=0.9$ $53534 \backslash \mathrm{S} 2 \mathrm{~A}=0.758641 \backslash \mathrm{RMSD}=9.751 \mathrm{e}-09 \backslash \mathrm{RMSF}=2.553 \mathrm{e}-06 \backslash \mathrm{Dipole}=-0.2845461,0.4$ $575775,-0.216963 \backslash \mathrm{PG}=\mathrm{C} 01 \quad[\mathrm{X}(\mathrm{C} 2 \mathrm{H} 5 \mathrm{O} 2)] \backslash \backslash @$

$16-) \mathrm{CH} 3 \mathrm{O}+(\mathrm{CH} 3) 2 \mathrm{C}=\mathrm{O} \rightarrow \mathrm{CH} 3 \mathrm{OOC}(\mathrm{CH} 3) 2$

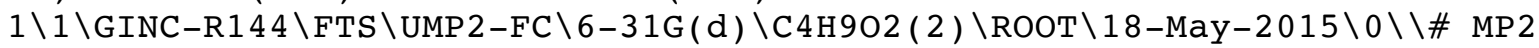
/6-31G(d) SCF=Tight INT(grid=ultrafine) OPT=(TS, calcfC, noeigentest, $\max$ cyc $=200) \operatorname{IOP}(2 / 17=4)$ maxdisk $=671088640 \backslash \backslash$ HS_diMetk.MP $2 \backslash \backslash 0,2 \backslash 0,-3.255514$ $8017,1.9163042504,1.273911253 \backslash \mathrm{C},-2.5613692405,3.0282376398,1.797390063$ $2 \backslash 0,-4.6151479445,1.9442501132,2.0172806073 \backslash \mathrm{C},-5.5814527159,1.77747500$ $62,1.1731125054 \backslash \mathrm{C},-5.8711145731,2.8574273861,0.1812952706 \backslash \mathrm{C},-6.1467474$ $287,0.4047490492,0.9942865635 \backslash \mathrm{H},-1.5619453598,2.9702159415,1.354282592$ $\backslash \mathrm{H},-3.0324398923,3.9764458964,1.5147915419 \backslash \mathrm{H},-2.4809051011,2.969037504$ $8,2.88788213 \backslash \mathrm{H},-7.209897542,0.4406229908,0.7414367614 \backslash \mathrm{H},-5.6269647641$, $-0.1094030157,0.1730340331 \backslash \mathrm{H},-6.0026741607,-0.1779378809,1.9054449984 \backslash$ $\mathrm{H},-6.9128421892,2.8277226654,-0.1482726787 \backslash \mathrm{H},-5.6541844906,3.834197657$ $5,0.6189157375 \backslash \mathrm{H},-5.2332357958,2.7310967955,-0.7032673786 \backslash \backslash$ Version=ES 6 $4 \mathrm{~L}-\mathrm{G} 09$ RevD.01 $\backslash$ State $=2-\mathrm{A} \backslash \mathrm{HF}=-306.2882942 \backslash \mathrm{MP} 2=-307.1282261 \backslash \mathrm{PUHF}=-306.311$ $4408 \backslash \mathrm{PMP} 2-0=-307.1480981 \backslash \mathrm{S} 2=0.989883 \backslash \mathrm{S} 2-1=0.920738 \backslash \mathrm{S} 2 \mathrm{~A}=0.75755 \backslash \mathrm{RMSD}=5$. $900 e-09 \backslash \mathrm{RMSF}=1.107 \mathrm{e}-06 \backslash \mathrm{Dipole}=-0.3455859,0.3182057,-0.3855137 \backslash \mathrm{PG}=\mathrm{C} 01$ [ $\mathrm{X}(\mathrm{C} 4 \mathrm{H} 9 \mathrm{O} 2)] \backslash \backslash @$

17-) $\mathrm{HS}+\mathrm{CH} 2=\mathrm{CH} 2->\mathrm{HSCH} 2 \mathrm{CH} 2$

$1 \backslash 1 \backslash G I N C-R 539 \backslash F T S \backslash U M P 2-F C \backslash 6-31 G(d) \backslash C 2 H 5 S 1(2) \backslash R O O T \backslash 14-M a y-2015 \backslash 0 \backslash \backslash \#$ MP2 /6-31G(d) SCF=Tight INT (grid=ultrafine) OPT=(TS, calcfC, noeigentest, $\max$ cyc $=200)$ IOP $(2 / 17=4)$ maxdisk $=402653184 \backslash \backslash$ HS_diMetk.MP $2 \backslash \backslash 0,2 \backslash S,-3.261843$ $7503,2.5450043949,0.9655844409 \backslash \mathrm{H},-2.8215708204,1.2965038139,1.18897984$ $13 \backslash \mathrm{C},-5.5266484312,1.8615061745,1.2512855531 \backslash \mathrm{C},-5.6320639531,0.9118138$ $372,0.2945363139 \backslash \mathrm{H},-5.8782043518,2.8696742847,1.0644784625 \backslash \mathrm{H},-5.409220$ $839,1.5910220083,2.2936804399 \backslash \mathrm{H},-5.4054899055,-0.1278003985,0.50110388$ $33 \backslash \mathrm{H},-5.8628539486,1.169009885,-0.731903935 \backslash \backslash$ Version=ES64L-G09RevD.01\ 
State $=2-\mathrm{A} \backslash \mathrm{HF}=-476.0864018 \backslash \mathrm{MP} 2=-476.4352372 \backslash \mathrm{PUHF}=-476.1018316 \backslash \mathrm{PMP} 2-0=-4$ $76.4483456 \backslash \mathrm{S} 2=0.953968 \backslash \mathrm{S} 2-1=0.891482 \backslash \mathrm{S} 2 \mathrm{~A}=0.756206 \backslash \mathrm{RMSD}=7.910 \mathrm{e}-09 \backslash \mathrm{RMSF}=$ $6.204 e-05 \backslash D i p o l e=-0.6583371,-0.6632403,0.0705711 \backslash P G=C 01 \quad[X(C 2 H 5 S 1)] \backslash \backslash @$

$18-) \mathrm{HS}+\mathrm{CH} 2=\mathrm{C}(\mathrm{CH} 3) 2->\mathrm{HSCH} 2 \mathrm{C}(\mathrm{CH} 3) 2$

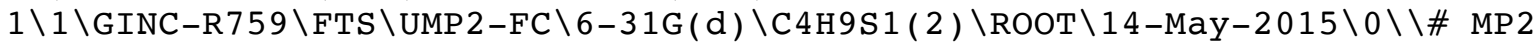
/6-31G(d) SCF=Tight INT(grid=ultrafine) OPT=(TS, calcfc, noeigentest, max

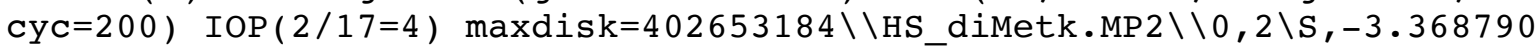
$1256,2.3378173963,0.845383601 \backslash \mathrm{H},-3.2096402 \overline{2} 46,1.3838385514,1.778587226$ $4 \backslash \mathrm{C},-5.7209363632,2.0334297276,0.9240003024 \backslash \mathrm{C},-5.8448652053,0.78182450$ $77,0.4194753709 \backslash \mathrm{H},-5.8961229166,2.8966680828,0.2908068863 \backslash \mathrm{H},-5.8045924$ $142,2.2095351255,1.9910361655 \backslash C,-5.7925917398,-0.4292667967,1.29879401$ $42 \backslash \mathrm{C},-5.9069358796,0.5321375933,-1.0548657655 \backslash \mathrm{H},-6.6663999464,-1.06702$ $05884,1.1235450499 \backslash \mathrm{H},-4.906888383,-1.0331292054,1.0667677004 \backslash \mathrm{H},-5.7644$ $107005,-0.1622583306,2.3577096254 \backslash \mathrm{H},-6.7759739698,-0.0880620859,-1.302$ $4095046 \backslash \mathrm{H},-5.9670506428,1.4645974999,-1.6194040776 \backslash \mathrm{H},-5.0148114885,-0$. $0123354775,-1.3850185947 \backslash \backslash$ Version=ES64L-G09RevD.01 State $=2-A \backslash H F=-554.1$ $653799 \backslash \mathrm{MP} 2=-554.7821031 \backslash \mathrm{PUHF}=-554.1793844 \backslash \mathrm{PMP} 2-0=-554.793879 \backslash \mathrm{S} 2=0.9273$ $83 \backslash \mathrm{S} 2-1=0.869954 \backslash \mathrm{S} 2 \mathrm{~A}=0.755132 \backslash \mathrm{RMSD}=9.613 \mathrm{e}-09 \backslash \mathrm{RMSF}=4.683 \mathrm{e}-05 \backslash \mathrm{Dipole}=-0$. $9769859,-0.81109,0.0872217 \backslash \mathrm{PG}=\mathrm{C} 01 \quad[\mathrm{X}(\mathrm{C} 4 \mathrm{H} 9 \mathrm{~S} 1)] \backslash \backslash @$

19-) $\mathrm{CH} 3 \mathrm{~S}+\mathrm{CH} 2=\mathrm{CH} 2 \rightarrow \mathrm{HSCH} 2 \mathrm{CH} 2$

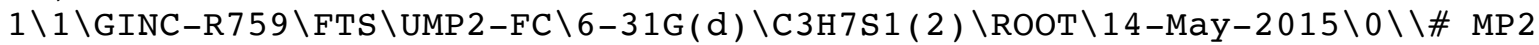
/6-31G(d) SCF=Tight INT(grid=ultrafine) OPT=(TS, calcfc, noeigentest, max

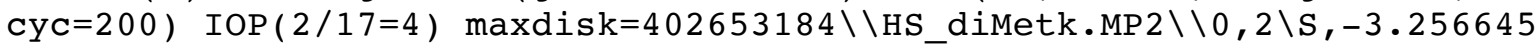
$661,2.8682779445,0.13524604 \backslash \mathrm{C},-2.801131859 \overline{3}, 2.7841975343,1.8836181236 \backslash$ $C,-5.5261496143,2.2371337078,0.4224730385 \backslash \mathrm{C},-5.4436986593,0.9992669106$ $, 0.9612501886 \backslash \mathrm{H},-5.7150479724,2.3616088286,-0.6377509229 \backslash \mathrm{H},-5.75317529$ $96,3.0964886589,1.043177981 \backslash \mathrm{H},-5.3895211254,0.8497878792,2.0332139111 \backslash$ $\mathrm{H},-5.3403682376,0.1191443924,0.3380819674 \backslash \mathrm{H},-1.7935354424,3.1988100282$ $, 1.9758911778 \backslash \mathrm{H},-3.4715921602,3.3810302293,2.5064662854 \backslash \mathrm{H},-2.779673968$ $5,1.7564798863,2.2489102093 \backslash \backslash$ Version=ES64L-G09RevD . 01 \State $=2-A \backslash H F=-51$ $5.1191679 \backslash \mathrm{MP} 2=-515.6011523 \backslash \mathrm{PUHF}=-515.1353883 \backslash \mathrm{PMP} 2-0=-515.6149782 \backslash \mathrm{S} 2=0$. $967122 \backslash \mathrm{S} 2-1=0.901765 \backslash \mathrm{S} 2 \mathrm{~A}=0.75713 \backslash \mathrm{RMSD}=9.202 \mathrm{e}-09 \backslash \mathrm{RMSF}=6.363 \mathrm{e}-06 \backslash \mathrm{Dipole}=$ $-0.4664116,-0.2774787,0.6273382 \backslash \mathrm{PG}=\mathrm{C} 01 \quad[\mathrm{X}(\mathrm{C} 3 \mathrm{H} 7 \mathrm{~S} 1)] \backslash \backslash @$

$20-) \mathrm{CH} 3 \mathrm{~S}+\mathrm{CH} 2=\mathrm{C}(\mathrm{CH} 3) 2->\operatorname{HSCH} 2 \mathrm{C}(\mathrm{CH} 3) 2$

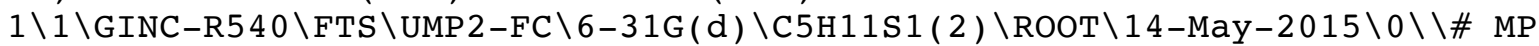
2/6-31G(d) SCF=Tight INT(grid=ultrafine) OPT=(TS, calcfc, noeigentest, ma

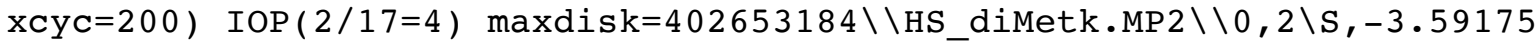
$26274,3.0707712235,0.2485184959 \backslash \mathrm{C},-3.014927 \overline{3} 936,2.4731178201,1.8557086$ $864 \backslash \mathrm{C},-5.8311214326,2.3630604372,0.4879527838 \backslash \mathrm{C},-5.7699259888,1.014498$ $0964,0.3670090177 \backslash \mathrm{H},-6.1343515031,2.9702069993,-0.358594196 \backslash \mathrm{H},-5.95947$ $63195,2.8224173189,1.4633231885 \backslash \mathrm{C},-5.5795496218,0.1180972971,1.5512156$ $641 \backslash \mathrm{C},-5.76344111,0.3500643796,-0.9746869738 \backslash \mathrm{H},-2.0126264663,2.8804838$ $604,2.016435758 \backslash \mathrm{H},-3.6533512614,2.8228335391,2.6709600868 \backslash \mathrm{H},-2.9454884$ $072,1.384349863,1.8909095325 \backslash \mathrm{H},-6.5189676343,-0.4429487029,-1.01726793$ $49 \backslash \mathrm{H},-5.9567897396,1.0635397718,-1.7783608677 \backslash \mathrm{H},-4.7904190783,-0.11978$ $38154,-1.1617836894 \backslash \mathrm{H},-6.3678361597,-0.6434505561,1.5830837859 \backslash \mathrm{H},-4.62$ $52951811,-0.4191727116,1.4824313513 \backslash \mathrm{H},-5.5973320753,0.6751821795,2.490$ $3873109 \backslash \backslash$ Version=ES64L-G09RevD.01 \State $=2-\mathrm{A} \backslash \mathrm{HF}=-593.196798 \backslash \mathrm{MP} 2=-593.94$ $74089 \backslash \mathrm{PUHF}=-593.2117259 \backslash \mathrm{PMP} 2-0=-593.9600142 \backslash \mathrm{S} 2=0.941701 \backslash \mathrm{S} 2-1=0.880919 \backslash$ $\mathrm{S} 2 \mathrm{~A}=0.756102 \backslash \mathrm{RMSD}=6.806 \mathrm{e}-09 \backslash \mathrm{RMSF}=2.377 \mathrm{e}-06 \backslash \mathrm{Dipole}=-0.5799198,-0.791622$ $3,0.4771242 \backslash \mathrm{PG}=\mathrm{C} 01 \quad[\mathrm{X}(\mathrm{C} 5 \mathrm{H} 11 \mathrm{~S} 1)] \backslash \backslash @$

21-) $\mathrm{CH} 3+\mathrm{H} 2 \mathrm{C}=\mathrm{S}->\mathrm{CH} 3 \mathrm{SCH} 2$

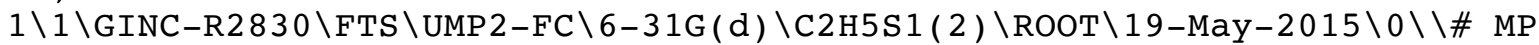
2/6-31G(d) SCF=Tight INT(grid=ultrafine) OPT=(TS, calcfc, noeigentest, ma

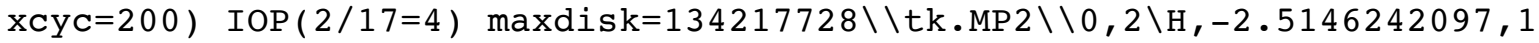
$.4568898455,0.2574972926 \backslash \mathrm{C},-2.7136999411,1.6705729995,1.2972796464 \backslash \mathrm{H},-$ 
$2.7245472202,0.8503391508,2.0008082 \backslash \mathrm{H},-2.5349796581,2.6684631228,1.669$ $7203753 \backslash \mathrm{S},-5.3836307769,1.7928238634,1.153639393 \backslash \mathrm{C},-5.8882255032,2.941$ $876861,0.1589338511 \backslash \mathrm{H},-6.0498630136,2.7618938501,-0.9029058709 \backslash \mathrm{H},-6.07$ $00846772,3.9620883068,0.4937771123 \backslash \backslash$ Version=ES64L-G09RevD.01 \State=2-A $\backslash \mathrm{HF}=-476.0642734 \backslash \mathrm{MP} 2=-476.4074271 \backslash \mathrm{PUHF}=-476.0823044 \backslash \mathrm{PMP} 2-0=-476.423525$ $5 \backslash \mathrm{S} 2=1.083242 \backslash \mathrm{S} 2-1=1.010041 \backslash \mathrm{S} 2 \mathrm{~A}=0.763836 \backslash \mathrm{RMSD}=9.771 \mathrm{e}-09 \backslash \mathrm{RMSF}=1.080 \mathrm{e}-06$ $\backslash$ Dipole $=0.2000048,0.4415603,-0.3765641 \backslash P G=C 01[X(C 2 H 5 S 1)] \backslash \backslash @$

$22-) \mathrm{CH} 3+(\mathrm{CH} 3) 2 \mathrm{C}=\mathrm{S}->\mathrm{CH} 3 \mathrm{SC}(\mathrm{CH} 3) 2$

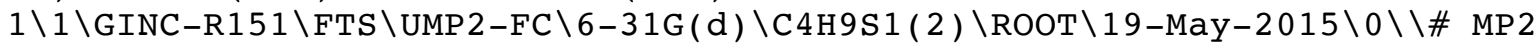
/6-31G(d) SCF=Tight INT(grid=ultrafine) OPT=(TS, calcfC, noeigentest, $\max$

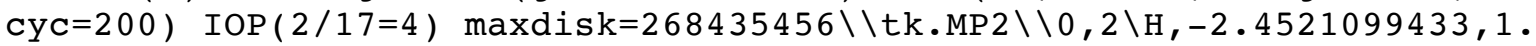
$6988708511,0.2796899918 \backslash \mathrm{C},-2.7664194807,1.6724804787,1.3135090021 \backslash \mathrm{H},-2$ $.7197702214,0.723305618,1.8299653335 \backslash \mathrm{H},-2.6603857583,2.57204339,1.9032$ $664695 \backslash \mathrm{S},-5.296470632,1.6415007871,1.0054561712 \backslash \mathrm{C},-5.8145085882,2.9657$ $658395,0.2269646702 \backslash \mathrm{C},-5.9009923181,3.0450739413,-1.2711827952 \backslash \mathrm{C},-6.18$ $48868525,4.2347950536,0.941527288 \backslash \mathrm{H},-5.5086566904,5.0445779286,0.63900$ $7251 \backslash \mathrm{H},-7.1974319552,4.5445775978,0.6569351412 \backslash \mathrm{H},-6.1350835242,4.11055$ $27491,2.0228561408 \backslash \mathrm{H},-6.9111796254,3.3451426383,-1.5739786822 \backslash \mathrm{H},-5.215$ $6405048,3.8168183668,-1.644355391 \backslash \mathrm{H},-5.6531089056,2.09043776,-1.734229$ $5909 \backslash \backslash$ Version=ES64L-G09RevD.01 \State $=2-\mathrm{A} \backslash \mathrm{HF}=-554.1454062 \backslash \mathrm{MP} 2=-554.7574$ $971 \backslash \mathrm{PUHF}=-554.1631729 \backslash \mathrm{PMP} 2-0=-554.7731462 \backslash \mathrm{S} 2=1.05165 \backslash \mathrm{S} 2-1=0.978005 \backslash \mathrm{S} 2 \mathrm{~A}$ $=0.762268 \backslash \mathrm{RMSD}=4.278 \mathrm{e}-09 \backslash \mathrm{RMSF}=8.093 \mathrm{e}-07 \backslash \mathrm{Dipole}=0.0763451,0.8104265,-0$. $4259433 \backslash P G=C 01 \quad[X(C 4 H 9 S 1)] \backslash \backslash @$

23-) $\mathrm{CH} 3 \mathrm{CH} 2+\mathrm{H} 2 \mathrm{C}=\mathrm{S}->\mathrm{CH} 3 \mathrm{CH} 2 \mathrm{SCH} 2$

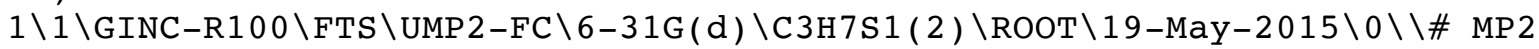
$16-31 \mathrm{G}(\mathrm{d}) \mathrm{SCF}=\mathrm{Tight}$ INT (grid=ultrafine) OPT=(TS, calcfC, noeigentest, $\max$ $\mathrm{Cyc}=200) \operatorname{IOP}(2 / 17=4)$ maxdisk $=134217728 \backslash \backslash \mathrm{tk} . \mathrm{MP} 2 \backslash \backslash 0,2 \backslash \mathrm{H},-2.4623552873,1$. $1637847572,0.3732393733 \backslash \mathrm{C},-2.6910604024,1.6766151607,1.2994046675 \backslash \mathrm{H},-2$ $.7564716864,1.0618095469,2.1888155366 \backslash \mathrm{C},-2.4135335209,3.1351206232,1.4$ $25265478 \backslash \mathrm{S},-5.415138678,1.6303405793,0.9107580264 \backslash \mathrm{C},-5.9080111637,2.95$ $13764899,0.1696610818 \backslash \mathrm{H},-5.9752251706,3.0214091499,-0.9157059086 \backslash \mathrm{H},-6$. $183355295,3.8560361075,0.7110999217 \backslash \mathrm{H},-2.7446882193,3.6794269924,0.536$ $152094 \backslash \mathrm{H},-2.9201951171,3.5604976477,2.294854613 \backslash \mathrm{H},-1.3377564592,3.3297$ $539453,1.5418071164 \backslash \backslash$ Version=ES64L-G09RevD.01 \State $=2-A \backslash H F=-515.102173$ $\backslash \mathrm{MP} 2=-515.5770039 \backslash \mathrm{PUHF}=-515.1191105 \backslash \mathrm{PMP} 2-0=-515.5921302 \backslash \mathrm{S} 2=1.064318 \backslash \mathrm{S} 2$ $-1=0.995422 \backslash \mathrm{S} 2 \mathrm{~A}=0.763169 \backslash \mathrm{RMSD}=6.731 \mathrm{e}-09 \backslash \mathrm{RMSF}=1.733 \mathrm{e}-06 \backslash \mathrm{Dipole}=0.248119$ $7,0.5957414,-0.2222503 \backslash P G=C 01[X(C 3 H 7 S 1)] \backslash \backslash @$

$24-) \mathrm{CH} 3 \mathrm{CH} 2+(\mathrm{CH} 3) 2 \mathrm{C}=\mathrm{S}->\mathrm{CH} 3 \mathrm{CH} 2 \mathrm{SC}(\mathrm{CH} 3) 2$

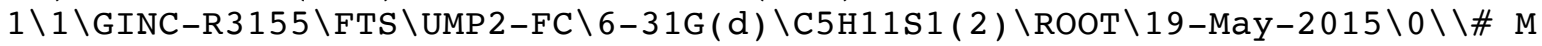
P2/6-31G(d) SCF=Tight INT(grid=ultrafine) OPT=(TS, calcfc, noeigentest, $\mathrm{m}$ axcyc $=200)$ IOP $(2 / 17=4)$ maxdisk=268435456\\HS_diMetk.MP $2 \backslash \backslash 0,2 \backslash \mathrm{H},-2.4843$ $625768,1.1513512721,0.3799434812 \backslash \mathrm{C},-2.765275 \overline{1} 352,1.6757786181,1.285673$ $7494 \backslash \mathrm{H},-2.7852393353,1.079023418,2.1903645831 \backslash \mathrm{C},-2.4827016908,3.136737$ $6415,1.3907421399 \backslash \mathrm{S},-5.3142994238,1.5857187742,0.9211107367 \backslash \mathrm{C},-5.81163$ $02277,2.9519749111,0.2114686255 \backslash \mathrm{C},-5.8616029352,3.1217529881,-1.281396$ $5028 \backslash \mathrm{C},-6.2191726348,4.1722291161,0.9893267692 \backslash \mathrm{H},-5.5673597571,5.01722$ $12824,0.7340538411 \backslash \mathrm{H},-7.2399409909,4.4656072287,0.7155398939 \backslash \mathrm{H},-6.1719$ $279935,3.9918179079,2.0628863919 \backslash \mathrm{H},-6.8693619861,3.4236579542,-1.59113$ $3071 \backslash \mathrm{H},-5.1813001472,3.9257396003,-1.5903423895 \backslash \mathrm{H},-5.5863669908,2.2007$ $867208,-1.7945096432 \backslash \mathrm{H},-2.7952419251,3.6652381929,0.48503083 \backslash \mathrm{H},-3.0054$ $984032,3.5792322832,2.2423160461 \backslash \mathrm{H},-1.4085678466,3.3293480903,1.524214$ $5185 \backslash \backslash$ Version=ES6 4L-G09RevD.01 \State $=2-A \backslash H F=-593.1829872 \backslash M P 2=-593.9274$ $692 \backslash \mathrm{PUHF}=-593.2003 \backslash \mathrm{PMP} 2-0=-593.9427267 \backslash \mathrm{S} 2=1.045079 \backslash \mathrm{S} 2-1=0.973289 \backslash \mathrm{S} 2 \mathrm{~A}=0$ $.762247 \backslash \mathrm{RMSD}=5.273 e-09 \backslash \mathrm{RMSF}=1.415 \mathrm{e}-06 \backslash \mathrm{Dipole}=0.1717147,0.8713932,-0.34$ $32611 \backslash \mathrm{PG}=\mathrm{C} 01 \quad[\mathrm{X}(\mathrm{C} 5 \mathrm{H} 11 \mathrm{~S} 1)] \backslash \backslash @$

25-) $\mathrm{HS}+\mathrm{H} 2 \mathrm{C}=\mathrm{S}->\mathrm{HSSCH} 2$ 
$1 \backslash 1 \backslash G I N C-R 3011 \backslash F T S \backslash U M P 2-F C \backslash 6-31 G(d) \backslash C 1 H 3 S 2(2) \backslash R O O T \backslash 27-M a r-2015 \backslash 0 \backslash \backslash \#$ MP 2/6-31G(d) SCF=Tight INT(grid=ultrafine) OPT=(TS, calcfC, noeigentest, ma $\mathrm{xcyc}=200) \operatorname{IOP}(2 / 17=4)$ maxdisk $=134217728 \backslash \backslash$ hstk_TS.MP $2 \backslash \backslash 0,2 \backslash \mathrm{S}, 0.25963119$ $27,0.2361638877,-0.5037152356 \backslash C, 1.3835554621, \overline{1} .1198842919,0.2061392736$ $\backslash \mathrm{S},-1.4952257012,1.9634210012,-1.4563474801 \backslash \mathrm{H},-2.4097651298,1.57374027$ $89,-0.5538539097 \backslash \mathrm{H}, 1.1411171597,1.9220969241,0.900098543 \backslash \mathrm{H}, 2.440814016$ $4,0.9670066161,-0.0011711913 \backslash \backslash$ Version=ES64L-G0 9RevD.01 \State $=2-\mathrm{A} \backslash \mathrm{HF}=-8$ $34.5645865 \backslash M P 2=-834.9021438 \backslash P U H F=-834.5812941 \backslash P M P 2-0=-834.916964 \backslash S 2=1$. $056918 \backslash \mathrm{S} 2-1=0.986088 \backslash \mathrm{S} 2 \mathrm{~A}=0.761042 \backslash \mathrm{RMSD}=6.920 \mathrm{e}-09 \backslash \mathrm{RMSF}=4.972 \mathrm{e}-06 \backslash \mathrm{Dipole}$ $=0.6378406,0.0305251,0.8317722 \backslash P G=C 01 \quad[X(C 1 H 3 S 2)] \backslash \backslash @$

$26-) \mathrm{HS}+(\mathrm{CH} 3) 2 \mathrm{C}=\mathrm{S} \rightarrow \mathrm{HSSC}(\mathrm{CH} 3) 2$

$1 \backslash 1 \backslash G I N C-R 3011 \backslash F T S \backslash U M P 2-F C \backslash 6-31 G(d) \backslash C 3 H 7 S 2(2) \backslash R O O T \backslash 27-M a r-2015 \backslash 0 \backslash \backslash \#$ MP 2/6-31G(d) SCF=Tight INT (grid=ultrafine) OPT=(TS, calcfC, noeigentest, ma $\mathrm{xcyc}=200) \quad \operatorname{IOP}(2 / 17=4)$ maxdisk $=134217728 \backslash \backslash$ HS_diMetk.MP $2 \backslash \backslash 0,2 \backslash \mathrm{S}, 0.313108$ $4153,0.153687965,-0.5662304491 \backslash \mathrm{C}, 1.3709205004,1.1741630044,0.120600512$ $7 \backslash \mathrm{S},-1.7360743863,1.5020402684,-1.123816212 \backslash \mathrm{H},-2.4214501112,1.01709459$ $58,-0.0759149869 \backslash \mathrm{C}, 1.0083321143,2.0766173696,1.2581119079 \backslash \mathrm{C}, 2.76758714$ $77,1.3268111807,-0.4031242854 \backslash \mathrm{H}, 3.4874917776,1.1224515506,0.3972103665$ $\backslash \mathrm{H}, 2.957367405,0.6609188232,-1.2440326311 \backslash \mathrm{H}, 2.9255556335,2.3658206063$, $-0.7191467106 \backslash \mathrm{H}, 1.7758176435,2.0255062108,2.0381996575 \backslash \mathrm{H}, 0.9803275309$, $3.1113409397,0.8950838049 \backslash \mathrm{H}, 0.0346923293,1.8277674854,1.6771000254 \backslash \backslash \mathrm{Ve}$ rsion=ES64L-G0 9RevD. $01 \backslash$ State $=2-A \backslash H F=-912.6470411 \backslash M P 2=-913.2564887 \backslash \mathrm{PUHF}$ $=-912.662243 \backslash \mathrm{PMP} 2-0=-913.2696669 \backslash \mathrm{S} 2=0.996361 \backslash \mathrm{S} 2-1=0.929449 \backslash \mathrm{S} 2 \mathrm{~A}=0.75833$ $1 \backslash \mathrm{RMSD}=9.221 \mathrm{e}-09 \backslash \mathrm{RMSF}=9.868 \mathrm{e}-07 \backslash \mathrm{Dipole}=1.2249752,0.3820278,1.0110393 \backslash \mathrm{P}$ $\mathrm{G}=\mathrm{C} 01[\mathrm{X}(\mathrm{C} 3 \mathrm{H} 7 \mathrm{~S} 2)] \backslash \backslash @$

27-) $\mathrm{CH} 3 \mathrm{~S}+\mathrm{H} 2 \mathrm{C}=\mathrm{S}->\mathrm{CH} 3 \mathrm{SSCH} 2$

$1 \backslash 1 \backslash G I N C-R 3398 \backslash F T S \backslash U M P 2-F C \backslash 6-31 G(d) \backslash C 2 H 5 S 2(2) \backslash R O O T \backslash 27-M a r-2015 \backslash 0 \backslash \backslash \#$ MP 2/6-31G(d) SCF=Tight INT(grid=ultrafine) OPT=(TS, calcfC, noeigentest, ma xcyc $=200)$ IOP $(2 / 17=4)$ maxdisk $=134217728 \backslash \backslash$ MeStk_TS.MP $2 \backslash \backslash 0,2 \backslash S, 0.3370361$ $272,0.1791029646,-0.4069506756 \backslash C, 1.5244682016, \overline{1} .0716555287,0.204463960$ $1 \backslash \mathrm{S},-1.4001753003,1.8736704007,-1.535656521 \backslash \mathrm{C},-2.5994429669,1.88459355$ $2,-0.1868362729 \backslash \mathrm{H}, 1.3559540463,1.8064285283,0.9892311341 \backslash \mathrm{H}, 2.545612051$ $3,0.9939025062,-0.16334498 \backslash \mathrm{H},-3.467175125,2.4530852907,-0.5368254503 \backslash \mathrm{H}$ $,-2.9260751052,0.8751597069,0.0647421003 \backslash \mathrm{H},-2.2012449288,2.3781485218$, $0.7005247052 \backslash \backslash$ Version=ES64L-G09RevD.01 \State $=2-\mathrm{A} \backslash \mathrm{HF}=-873.6024003 \backslash \mathrm{MP} 2=-$ $874.0697462 \backslash \mathrm{PUHF}=-873.6202913 \backslash \mathrm{PMP} 2-0=-874.0856456 \backslash \mathrm{S} 2=1.080142 \backslash \mathrm{S} 2-1=1.0$ $04966 \backslash \mathrm{S} 2 \mathrm{~A}=0.762628 \backslash \mathrm{RMSD}=4.533 \mathrm{e}-09 \backslash \mathrm{RMSF}=3.434 \mathrm{e}-06 \backslash \mathrm{Dipole}=0.2471658,0.32$ $45291,0.8327049 \backslash \mathrm{PG}=\mathrm{C} 01 \quad[\mathrm{X}(\mathrm{C} 2 \mathrm{H} 5 \mathrm{~S} 2)] \backslash \backslash @$

28-) $\mathrm{CH} 3 \mathrm{~S}+(\mathrm{CH} 3) 2 \mathrm{C}=\mathrm{S}->\mathrm{CH} 3 \mathrm{SSC}(\mathrm{CH} 3) 2$

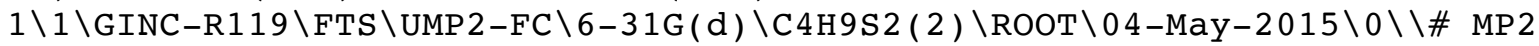
/6-31G(d) SCF=Tight INT(grid=ultrafine) OPT=(TS, calcfC, noeigentest, $\max$ cyc $=200)$ IOP $(2 / 17=4)$ maxdisk=939524096\\HS_diMetk.MP $2 \backslash \backslash 0,2 \backslash S, 0.3470407$ $681,0.3342442349,-0.8686228027 \backslash C, 1.4116495 \overline{1} 08,1.223822438,-0.021911575$ $3 \backslash S,-1.8143390597,1.7050692778,-0.9657292511 \backslash \mathrm{C},-2.6915239146,0.8612501$ $621,0.3706253962 \backslash \mathrm{C}, 1.151362419,1.6636042727,1.3864712091 \backslash \mathrm{C}, 2.679590037$ $7,1.7420522967,-0.6311319431 \backslash \mathrm{H}, 3.5405474236,1.3998607456,-0.045519204 \backslash$ $\mathrm{H}, 2.7889708026,1.4176287118,-1.6651676423 \backslash \mathrm{H}, 2.6817356996,2.8389622129$, $-0.592031239 \backslash \mathrm{H}, 2.0598379236,1.5802078837,1.9924490873 \backslash \mathrm{H}, 0.8615113347,2$ $.7221539514,1.374633127 \backslash \mathrm{H}, 0.3477682613,1.0870768481,1.8433817895 \backslash \mathrm{H},-3$. $7263813156,1.2171221839,0.3526613085 \backslash \mathrm{H},-2.6963002122,-0.2190315621,0.2$ $193437369 \backslash \mathrm{H},-2.2650726788,1.0924013427,1.3481280028 \backslash \backslash$ Version=ES64L-G09 RevD. $01 \backslash$ State $=2-A \backslash H F=-951.6831999 \backslash M P 2=-952.4232513 \backslash P U H F=-951.6994806 \backslash P$ MP2 $-0=-952.4374449 \backslash \mathrm{S} 2=1.020736 \backslash \mathrm{S} 2-1=0.949834 \backslash \mathrm{S} 2 \mathrm{~A}=0.759735 \backslash \mathrm{RMSD}=4.541 \mathrm{e}-$ $09 \backslash \mathrm{RMSF}=4.782 \mathrm{e}-06 \backslash \mathrm{Dipole}=0.8356172,0.2410824,1.0754251 \backslash \mathrm{PG}=\mathrm{C} 01 \quad[\mathrm{X}(\mathrm{C} 4 \mathrm{H} 9 \mathrm{~S}$ 2 ) $] \backslash \backslash @$

\section{Products}


Product $1-) \mathrm{CH} 3 \mathrm{CH} 2 \mathrm{CH} 2$

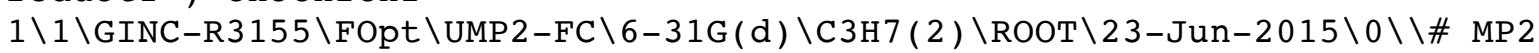
$16-31 \mathrm{G}(\mathrm{d}) \quad \mathrm{SCF}=\mathrm{Tight}$ INT(grid=ultrafine) OPT IOP $(2 / 17=4)$ maxdisk $=134217$ $728 \backslash \backslash \mathrm{tk} . \mathrm{MP} 2 \backslash \backslash 0,2 \backslash \mathrm{H},-2.4568266283,1.7890034576,0.0609083036 \backslash \mathrm{C},-2.917240$ $1426,1.8819442125,1.048021767 \backslash \mathrm{H},-2.599799223,1.0245855472,1.6491198922$ $\backslash \mathrm{H},-2.529599806,2.7878934157,1.5223272107 \backslash \mathrm{C},-4.4412326656,1.9264724514$ $, 0.9320512795 \backslash \mathrm{C},-5.0105314972,0.6956585112,0.3094793838 \backslash \mathrm{H},-4.743420945$ $3,2.8059736021,0.3512036793 \backslash \mathrm{H},-4.8708159208,2.0757887281,1.936109297 \backslash \mathrm{H}$ $,-5.9941573568,0.7034241034,-0.1437717063 \backslash \mathrm{H},-4.5603768144,-0.270690029$ $1,0.5054938932 \backslash \backslash$ Version=ES $64 \mathrm{~L}-\mathrm{G} 09$ RevD.01\State $=2-\mathrm{A} \backslash \mathrm{HF}=-117.6313291 \backslash \mathrm{MP} 2$ $=-118.0004657 \backslash \mathrm{PUHF}=-117.6345742 \backslash \mathrm{PMP} 2-0=-118.0025627 \backslash \mathrm{S} 2=0.762599 \backslash \mathrm{S} 2-1=0$ $.753638 \backslash \mathrm{S} 2 \mathrm{~A}=0.750107 \backslash \mathrm{RMSD}=2.803 \mathrm{e}-09 \backslash \mathrm{RMSF}=1.350 \mathrm{e}-05 \backslash \mathrm{Dipole}=-0.0176469,0$ $.0499612,0.0800586 \backslash P G=\mathrm{C} 01 \quad[\mathrm{X}(\mathrm{C} 3 \mathrm{H} 7)] \backslash \backslash @$

Product 2-) $\mathrm{CH} 3 \mathrm{CH} 2 \mathrm{C}(\mathrm{CH} 3) 2$

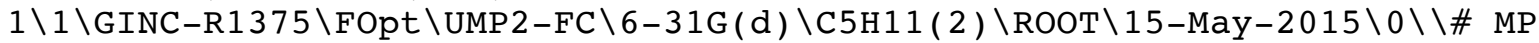
2/6-31G(d) SCF=Tight INT (grid=ultrafine) OPT IOP $(2 / 17=4$ ) maxdisk=53687 $0912 \backslash \backslash t \mathrm{k} . \mathrm{MP} 2 \backslash \backslash 0,2 \backslash \mathrm{H},-2.4868380418,1.8982839743,0.0596994576 \backslash \mathrm{C},-2.87805$ $37607,1.9537144885,1.0795512589 \backslash \mathrm{H},-2.4867709104,1.0993724187,1.6393031$ $469 \backslash \mathrm{H},-2.4896495458,2.8667219273,1.5413038805 \backslash \mathrm{C},-4.4169551332,1.949120$ $272,1.0774056114 \backslash \mathrm{C},-5.0034906191,0.7188331306,0.4551473189 \backslash \mathrm{H},-4.772543$ $0076,2.837230644,0.5395901392 \backslash \mathrm{H},-4.7724060344,2.0421143921,2.111542010$ $2 \backslash C,-5.0123020603,-0.5490380863,1.2488167735 \backslash C,-5.0153450728,0.6080315$ $298,-1.036509978 \backslash \mathrm{H},-5.7564092951,-1.253555403,0.8617898301 \backslash \mathrm{H},-4.038973$ $2365,-1.0651785198,1.2120026589 \backslash \mathrm{H},-5.2366809725,-0.3586265147,2.303385$ $9967 \backslash \mathrm{H},-5.7596986808,-0.1214143189,-1.3736832969 \backslash \mathrm{H},-5.2413141237,1.570$ $5620119,-1.5068046632 \backslash \mathrm{H},-4.0426375053,0.2734300536,-1.4327691446 \backslash \backslash$ Vers ion $=\mathrm{ES} 64 \mathrm{~L}-\mathrm{G} 09$ RevD. $01 \backslash$ State $=2-\mathrm{A} \backslash \mathrm{HF}=-195.7075266 \backslash \mathrm{MP} 2=-196.3416813 \backslash \mathrm{PUHF}=-$ $195.7109628 \backslash \mathrm{PMP} 2-0=-196.3438839 \backslash \mathrm{S} 2=0.76357 \backslash \mathrm{S} 2-1=0.753763 \backslash \mathrm{S} 2 \mathrm{~A}=0.750147 \backslash$ $\mathrm{RMSD}=5.582 \mathrm{e}-09 \backslash \mathrm{RMSF}=2.536 \mathrm{e}-05 \backslash \mathrm{Dipole}=0.0991146,-0.0236276,-0.0120909 \backslash \mathrm{P}$ $\mathrm{G}=\mathrm{C} 01[\mathrm{X}(\mathrm{C} 5 \mathrm{H} 11)] \backslash \backslash @$

Product 3-) $\mathrm{CH} 3 \mathrm{CH} 2 \mathrm{CH} 2 \mathrm{CH} 2$

$1 \backslash 1 \backslash G I N C-R 3580 \backslash F O p t \backslash U M P 2-F C \backslash 6-31 G(d) \backslash C 4 H 9(2) \backslash R O O T \backslash 23-J u n-2015 \backslash 0 \backslash \backslash \#$ MP2 $/ 6-31 \mathrm{G}(\mathrm{d}) \quad \mathrm{SCF}=\mathrm{Tight}$ INT (grid=ultrafine) OPT IOP $(2 / 17=4$ ) maxdisk=134217 $728 \backslash \backslash \mathrm{tk} . \mathrm{MP} 2 \backslash \backslash 0,2 \backslash \mathrm{H},-2.4812895677,1.7795075601,0.0556149084 \backslash \mathrm{C},-2.915356$ $3073,1.8986969537,1.0548156571 \backslash \mathrm{H},-2.6189464087,1.0126760068,1.62962877$ $38 \backslash \mathrm{C},-2.3552050249,3.1535034846,1.7179135485 \backslash \mathrm{C},-4.440756057,1.92709319$ $23,0.9347802195 \backslash \mathrm{C},-5.0044599997,0.6936201059,0.3130400929 \backslash \mathrm{H},-4.7439326$ $409,2.8080507882,0.3544980377 \backslash \mathrm{H},-4.8673511363,2.0770203505,1.941475243$ $1 \backslash \mathrm{H},-5.9773395118,0.7007244917,-0.1628105803 \backslash \mathrm{H},-4.5593779261,-0.272450$ $6176,0.5213748093 \backslash \mathrm{H},-2.6193889982,4.0479534149,1.1451857529 \backslash \mathrm{H},-2.75726$ $20935,3.273421959,2.7288100352 \backslash \mathrm{H},-1.264709328,3.1131093099,1.792560501$ $9 \backslash \backslash$ Version=ES6 4L-G0 9RevD.01 \State $=2-\mathrm{A} \backslash \mathrm{HF}=-156.6660319 \backslash \mathrm{MP} 2=-157.1662085$ $\backslash \mathrm{PUHF}=-156.66929 \backslash \mathrm{PMP} 2-0=-157.1683132 \backslash \mathrm{S} 2=0.762643 \backslash \mathrm{S} 2-1=0.753646 \backslash \mathrm{S} 2 \mathrm{~A}=0.7$ $50108 \backslash \mathrm{RMSD}=9.134 \mathrm{e}-09 \backslash \mathrm{RMSF}=7.761 \mathrm{e}-07 \backslash \mathrm{Dipole}=0.0010021,0.0391819,0.07101$ $57 \backslash P G=C 01 \quad[X(\mathrm{C} 4 \mathrm{H} 9)] \backslash \backslash @$

Product $4-)$ CH3CH $2 \mathrm{CH} 2 \mathrm{C}(\mathrm{CH} 3) 2$

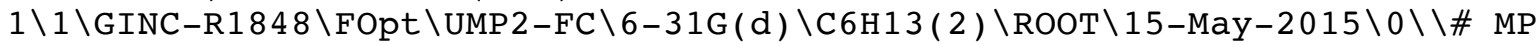
2/6-31G(d) SCF=Tight INT(grid=ultrafine) OPT IOP $(2 / 17=4)$ maxdisk=53687 $0912 \backslash \backslash t \mathrm{tk} . \mathrm{MP} 2 \backslash \backslash 0,2 \backslash \mathrm{H},-2.6120376181,1.4699862077,0.1751015733 \backslash \mathrm{C},-3.04262$ $15018,1.7289438607,1.1497191163 \backslash \mathrm{H},-2.9001671771,0.8552856771,1.7968242$ $605 \backslash C,-2.3060086293,2.9301164235,1.7359816864 \backslash C,-4.5508036624,1.992622$ $953,0.9816879664 \backslash \mathrm{C},-5.3043315853,0.83315663,0.4067224081 \backslash \mathrm{H},-4.67877683$ $65,2.8739398058,0.3379462556 \backslash \mathrm{H},-4.967920683,2.2556155605,1.963756559 \backslash \mathrm{C}$ $,-5.6242048562,-0.3251060997,1.2973443186 \backslash \mathrm{C},-5.215382245,0.5799054861$, $-1.0646887466 \backslash \mathrm{H},-2.416750603,3.8071078205,1.0905832005 \backslash \mathrm{H},-2.7058431409$ , $3.1882982984,2.7215726458 \backslash \mathrm{H},-1.2366051243,2.7284328121,1.8490197666 \backslash \mathrm{H}$ 
$,-6.0560704055,-0.0297179723,-1.413645009 \backslash \mathrm{H},-5.21277994,1.5166547273,-$ $1.6314639633 \backslash \mathrm{H},-4.2959915134,0.0347287878,-1.3342958964 \backslash \mathrm{H},-6.457789774$ $8,-0.9135512613,0.8985306788 \backslash \mathrm{H},-4.7703967365,-1.0148614653,1.399232397$ $4 \backslash \mathrm{H},-5.8915069668,0.0084077479,2.3053527822 \backslash \backslash$ Version=ES64L-G09RevD.01\} State $=2-A \backslash H F=-234.742217 \backslash M P 2=-235.5077243 \backslash P U H F=-234.7456774 \backslash P M P 2-0=-23$ $5.5099423 \backslash \mathrm{S} 2=0.763676 \backslash \mathrm{S} 2-1=0.753792 \backslash \mathrm{S} 2 \mathrm{~A}=0.750149 \backslash \mathrm{RMSD}=7.179 \mathrm{e}-09 \backslash \mathrm{RMSF}=1$ $.014 e-05 \backslash \mathrm{Dipole}=0.1094421,-0.0547021,-0.0017859 \backslash \mathrm{PG}=\mathrm{C} 01[\mathrm{X}(\mathrm{C} 6 \mathrm{H} 13)] \backslash \backslash @$

Product 5-) $\mathrm{CH} 3 \mathrm{OCH} 2$

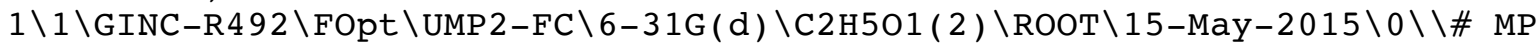
2/6-31G(d) SCF=Tight INT(grid=ultrafine) OPT IOP(2/17=4) maxdisk=26843 $5456 \backslash \backslash \mathrm{tk}$.MP $2 \backslash \backslash 0,2 \backslash \mathrm{H},-2.731820251,0.6113905614,0.5590947388 \backslash \mathrm{C},-3.014103$ $0972,1.5617218773,1.0227653861 \backslash \mathrm{H},-2.7927715718,1.5324383824,2.08906331$ $26 \backslash \mathrm{H},-2.4514691456,2.3768797778,0.5524589174 \backslash \mathrm{O},-4.4173996134,1.7850009$ $042,0.9072612373 \backslash \mathrm{C},-4.8432819267,1.9062432486,-0.3836720804 \backslash \mathrm{H},-4.15643$ $10028,2.3550582162,-1.0986975234 \backslash \mathrm{H},-5.9012893914,2.1220200323,-0.44993$ 99885 \Version=ES64L-G09RevD.01 \State=2-A \HF=-153.4358888 \MP2=-153.852 $1389 \backslash \mathrm{PUHF}=-153.4385751 \backslash \mathrm{PMP} 2-0=-153.8537836 \backslash \mathrm{S} 2=0.759234 \backslash \mathrm{S} 2-1=0.752046 \backslash \mathrm{S}$ $2 \mathrm{~A}=0.75006 \backslash \mathrm{RMSD}=3.253 \mathrm{e}-09 \backslash \mathrm{RMSF}=2.847 \mathrm{e}-05 \backslash \mathrm{Dipole}=0.520029,0.0960816,-0$. $1716389 \backslash \mathrm{PG}=\mathrm{C} 01 \quad[\mathrm{X}(\mathrm{C} 2 \mathrm{H} 5 \mathrm{O} 1)] \backslash \backslash @$

Product 6-) $\mathrm{CH} 3 \mathrm{OC}(\mathrm{CH} 3) 2$

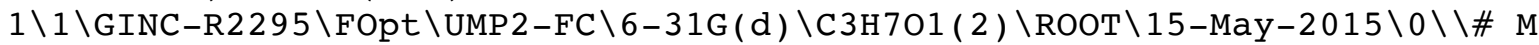
P2/6-31G(d) SCF=Tight INT(grid=ultrafine) OPT IOP(2/17=4) maxdisk=5368 $70912 \backslash \backslash \mathrm{tk} . \mathrm{MP} 2 \backslash \backslash 0,2 \backslash \mathrm{H},-2.690736834,2.1083551084,-0.1831268134 \backslash \mathrm{C},-2.9244$ $280406,1.9318451309,0.8738251224 \backslash \mathrm{H},-2.3523559635,1.0547740954,1.207044$ $9864 \backslash \mathrm{C},-2.6079964922,3.1455212909,1.7188688974 \backslash 0,-4.3245620984,1.66353$ $63868,1.0127052512 \backslash \mathrm{C},-4.7420847465,0.5485564576,0.3445090829 \backslash \mathrm{H},-5.7908$ $430632,0.3379646966,0.5075446668 \backslash \mathrm{H},-4.0363438325,-0.2746034025,0.25193$ $21381 \backslash \mathrm{H},-3.1918212281,4.0033563195,1.3792028447 \backslash \mathrm{H},-2.851696667,2.95138$ $82876,2.7654092286 \backslash \mathrm{H},-1.545363034,3.3929916287,1.645270595 \backslash \backslash$ Version=ES $64 \mathrm{~L}-\mathrm{G} 09$ RevD . $01 \backslash$ State $=2-\mathrm{A} \backslash \mathrm{HF}=-192.4759431 \backslash \mathrm{MP} 2=-193.0231754 \backslash \mathrm{PUHF}=-192.47$ $86348 \backslash \mathrm{PMP} 2-0=-193.0248243 \backslash \mathrm{S} 2=0.759274 \backslash \mathrm{S} 2-1=0.75206 \backslash \mathrm{S} 2 \mathrm{~A}=0.750061 \backslash \mathrm{RMSD}=6$ $.903 e-09 \backslash \mathrm{RMSF}=3.161 \mathrm{e}-05 \backslash \mathrm{Dipole}=0.5042986,-0.1319726,-0.0010686 \backslash \mathrm{PG}=\mathrm{C} 01$ $[\mathrm{X}(\mathrm{C} 3 \mathrm{H} 701)] \backslash \backslash @$

Product 7-) $\mathrm{CH} 3 \mathrm{OC}(\mathrm{CH} 3) 2$

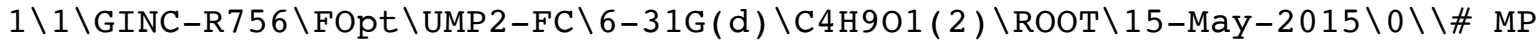
2/6-31G(d) SCF=Tight INT(grid=ultrafine) OPT IOP(2/17=4) maxdisk=26843 $5456 \backslash \backslash t \mathrm{k} . M P 2 \backslash \backslash 0,2 \backslash \mathrm{H},-2.2462380855,2.5099842389,0.6944572386 \backslash \mathrm{C},-3.04568$ $21863,1.7706539096,0.6419254735 \backslash \mathrm{H},-3.6603719454,1.9602666099,-0.244620$ $8519 \backslash \mathrm{H},-2.6046066298,0.7707875079,0.5717579543 \backslash \mathrm{O},-3.8043124921,1.93404$ $68065,1.837103607 \backslash \mathrm{C},-4.9968711942,1.2346421254,1.9012997047 \backslash \mathrm{C},-5.72840$ $35083,1.5865204197,3.1540956128 \backslash \mathrm{C},-5.0176957838,-0.1834872571,1.417191$ $1961 \backslash \mathrm{H},-6.0277874308,-0.588288909,1.5161559596 \backslash \mathrm{H},-4.724154473,-0.27727$ $40023,0.3682864473 \backslash \mathrm{H},-4.3421167978,-0.8193723398,2.0113182897 \backslash \mathrm{H},-6.758$ $5835579,1.22298986,3.1138996438 \backslash \mathrm{H},-5.2473478535,1.1434770382,4.0392733$ $53 \backslash \mathrm{H},-5.7407730616,2.6705829922,3.2905813714 \backslash \backslash$ Version=ES64L-G09RevD 01 $\backslash$ State $=2-A \backslash H F=-231.5166935 \backslash M P 2=-232.1960761 \backslash \mathrm{PUHF}=-231.5195036 \backslash \mathrm{PMP} 2-0=-$ $232.1978143 \backslash \mathrm{S} 2=0.76022 \backslash \mathrm{S} 2-1=0.752386 \backslash \mathrm{S} 2 \mathrm{~A}=0.750082 \backslash \mathrm{RMSD}=9.048 \mathrm{e}-09 \backslash \mathrm{RMSF}=$ $1.512 e-05 \backslash \mathrm{Dipole}=0.0763201,-0.3356608,-0.3541043 \backslash \mathrm{PG}=\mathrm{C} 01 \quad[\mathrm{X}(\mathrm{C} 4 \mathrm{H} 901)] \backslash \backslash @$

Product $8-) \mathrm{CH} 3 \mathrm{CH} 2 \mathrm{OC}(\mathrm{CH} 3) 2$

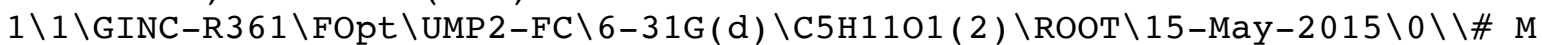
P2/6-31G(d) SCF=Tight INT(grid=ultrafine) OPT IOP(2/17=4) maxdisk=5368 $70912 \backslash \backslash \mathrm{k} . \mathrm{MP} 2 \backslash \backslash 0,2 \backslash \mathrm{H},-2.5756913939,0.9137006873,0.978815357 \backslash \mathrm{C},-3.10655$ $15827,1.7413434771,1.4660071377 \backslash \mathrm{H},-3.226140141,1.4923600565,2.52819206$ $4 \backslash \mathrm{C},-2.3470245181,3.0392714258,1.2925950332 \backslash 0,-4.3922508199,1.93054430$ $95,0.8665683284 \backslash \mathrm{C},-5.34032883,0.9517603863,1.1118112454 \backslash \mathrm{C},-4.914260529$ $8,-0.4833063928,1.0431261023 \backslash \mathrm{C},-6.6524563476,1.3508818193,0.5221458873$ 
$\backslash \mathrm{H},-2.2591259566,3.2834588333,0.2317414935 \backslash \mathrm{H},-2.8742138649,3.853868216$ $7,1.7932116148 \backslash \mathrm{H},-1.3426914243,2.9543376139,1.7175159383 \backslash \mathrm{H},-7.45365287$ $07,0.7054196786,0.8918205398 \backslash \mathrm{H},-6.8831641581,2.3864813493,0.7837432175$ $\backslash \mathrm{H},-6.639136726,1.2786289153,-0.5760235104 \backslash \mathrm{H},-5.7631284913,-1.12912880$ $17,1.2810504837 \backslash \mathrm{H},-4.5636894673,-0.7484835169,0.0329057216 \backslash \mathrm{H},-4.110051$ $8778,-0.7187250577,1.745230346 \backslash \backslash$ Version=ES64L-G09RevD.01 $\backslash$ State $=2-A \backslash H F=$ $-270.5565467 \backslash \mathrm{MP} 2=-271.3672499 \backslash \mathrm{PUHF}=-270.5593589 \backslash \mathrm{PMP} 2-0=-271.36899 \backslash \mathrm{S} 2=0$ $.760241 \backslash \mathrm{S} 2-1=0.752395 \backslash \mathrm{S} 2 \mathrm{~A}=0.750083 \backslash \mathrm{RMSD}=5.118 \mathrm{e}-09 \backslash \mathrm{RMSF}=1.933 \mathrm{e}-05 \backslash \mathrm{Dipol}$ $e=0.2866743,-0.3159933,0.1276675 \backslash P G=C 01[X(C 5 H 1101)] \backslash \backslash @$

\section{Product 9-) НОСН $2 \mathrm{CH} 2$}

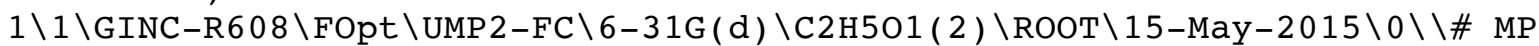
2/6-31G(d) SCF=Tight INT(grid=ultrafine) OPT IOP(2/17=4) maxdisk=53687 $0912 \backslash \backslash t \mathrm{k} . \mathrm{MP} 2 \backslash \backslash 0,2 \backslash \mathrm{O},-2.8953886534,2.5899652692,0.9121547134 \backslash \mathrm{H},-2.24819$ $51864,1.8773935149,1.0476039806 \backslash \mathrm{C},-4.1784482185,2.0337835461,1.2114571$ $623 \backslash \mathrm{C},-4.4863401633,0.8387654887,0.3785319955 \backslash \mathrm{H},-4.8848912379,2.851043$ $6408,1.0157865811 \backslash \mathrm{H},-4.2670121288,1.7881431774,2.2801187676 \backslash \mathrm{H},-5.21502$ $57215,0.1065682242,0.7037007912 \backslash \mathrm{H},-4.1743166902,0.8294361387,-0.658398$

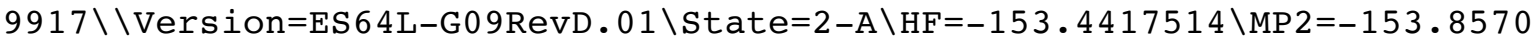
$67 \backslash \mathrm{PUHF}=-153.4449062 \backslash \mathrm{PMP} 2-0=-153.859101 \backslash \mathrm{S} 2=0.762148 \backslash \mathrm{S} 2-1=0.753474 \backslash \mathrm{S} 2 \mathrm{~A}=$ $0.7501 \backslash \mathrm{RMSD}=7.916 \mathrm{e}-09 \backslash \mathrm{RMSF}=3.122 \mathrm{e}-05 \backslash \mathrm{Dipole}=-0.1785059,-0.6267804,0.17$ $07783 \backslash \mathrm{PG}=\mathrm{C} 01 \quad[\mathrm{X}(\mathrm{C} 2 \mathrm{H} 5 \mathrm{O} 1)] \backslash \backslash \mathrm{Q}$

\section{Product $10-)$ HOCH $2 \mathrm{C}(\mathrm{CH} 3) 2$}

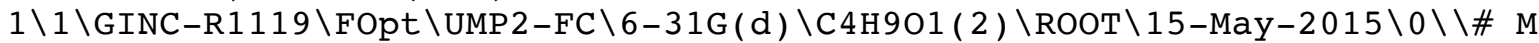
P2/6-31G(d) SCF=Tight INT(grid=ultrafine) OPT IOP(2/17=4) maxdisk=5368 $70912 \backslash \backslash \mathrm{k} . \mathrm{MP} 2 \backslash \backslash 0,2 \backslash 0,-2.9468931764,2.3669566411,0.8856648556 \backslash \mathrm{H},-2.5070$ $097445,1.6644567123,1.3943696095 \backslash \mathrm{C},-4.36918016,2.1364890674,1.01021716$ $04 \backslash \mathrm{C},-4.7919736364,0.8397252188,0.4021485435 \backslash \mathrm{H},-4.824071428,2.97931195$ $68,0.4823323326 \backslash \mathrm{H},-4.6743607486,2.1912313456,2.064957896 \backslash \mathrm{C},-4.72743622$ $96,-0.4059098212,1.2282058989 \backslash \mathrm{C},-4.6884994727,0.71129391,-1.0830441349$ $\backslash \mathrm{H},-5.280369981,-0.1312200403,-1.4546337587 \backslash \mathrm{H},-5.0278984277,1.62285850$ $16,-1.5846140349 \backslash \mathrm{H},-3.6462347118,0.5448013493,-1.3930291963 \backslash \mathrm{H},-5.39512$ $36125,-1.1780896169,0.832236241 \backslash \mathrm{H},-3.7152773226,-0.8431438225,1.232723$ $7436 \backslash \mathrm{H},-5.0053433481,-0.213612402,2.2694128437 \backslash \backslash$ Version=ES64L-G09RevD. $01 \backslash$ State $=2-A \backslash H F=-231.5181848 \backslash M P 2=-232.1982177 \backslash P U H F=-231.5216456 \backslash P M P 2-0$ $=-232.2004459 \backslash \mathrm{S} 2=0.763748 \backslash \mathrm{S} 2-1=0.75389 \backslash \mathrm{S} 2 \mathrm{~A}=0.750152 \backslash \mathrm{RMSD}=8.102 \mathrm{e}-09 \backslash \mathrm{RMS}$ $\mathrm{F}=1.826 \mathrm{e}-05 \backslash \mathrm{Dipole}=-0.2038308,-0.6221661,0.2821647 \backslash \mathrm{PG}=\mathrm{C} 01[\mathrm{X}(\mathrm{C} 4 \mathrm{H} 9 \mathrm{O} 1)] \backslash$ $\backslash \mathrm{Q}$

\section{Product $11-) \mathrm{CH} 3 \mathrm{OCH} 2 \mathrm{CH} 2$}

$1 \backslash 1 \backslash G I N C-R 1154 \backslash F O p t \backslash U M P 2-F C \backslash 6-31 G(d) \backslash C 3 H 7 O 1(2) \backslash R O O T \backslash 15-M a y-2015 \backslash 0 \backslash \backslash \# M$ P2/6-31G(d) SCF=Tight INT(grid=ultrafine) OPT IOP(2/17=4) maxdisk=5368 $70912 \backslash \backslash t k$.MP2 $\backslash \backslash 0,2 \backslash 0,-3.1362716407,2.0235055146,2.1806932825 \backslash \mathrm{C},-2.4468$ $531797,3.0065768618,1.4227640127 \backslash \mathrm{C},-4.4612733571,1.8187956235,1.702376$ $7456 \backslash \mathrm{C},-4.5000653897,1.1347600131,0.3784882836 \backslash \mathrm{H},-5.0061060901,2.77597$ $04879,1.6586411411 \backslash \mathrm{H},-4.9456549361,1.2075291339,2.4769328444 \backslash \mathrm{H},-3.7576$ $378831,0.3776254673,0.1589134342 \backslash \mathrm{H},-5.3856170611,1.1828453872,-0.24321$ $3695 \backslash \mathrm{H},-1.4646016424,3.119472218,1.8830763405 \backslash \mathrm{H},-2.3248175145,2.706139$ $3488,0.3750837663 \backslash \mathrm{H},-2.9738913055,3.9707589438,1.452436844 \backslash \backslash$ Version=ES 64L-G09RevD.01 SState $=2-A \backslash H F=-192.468743 \backslash M P 2=-193.0127454 \backslash P U H F=-192.471$ $9225 \backslash \mathrm{PMP} 2-0=-193.0147937 \backslash \mathrm{S} 2=0.762214 \backslash \mathrm{S} 2-1=0.753481 \backslash \mathrm{S} 2 \mathrm{~A}=0.7501 \backslash \mathrm{RMSD}=7.3$ $18 \mathrm{e}-09 \backslash \mathrm{RMSF}=7.204 \mathrm{e}-06 \backslash \mathrm{Dipole}=-0.2491478,0.2097549,-0.4073458 \backslash \mathrm{PG}=\mathrm{C} 01] \mathrm{X}$ ( C3H701) ] \\@

Product 12-) $\mathrm{CH} 3 \mathrm{OCH} 2 \mathrm{C}(\mathrm{CH} 3) 2$

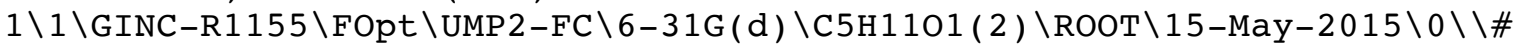
MP 2/6-31G(d) SCF=Tight INT(grid=ultrafine) OPT IOP $(2 / 17=4)$ maxdisk $=536$ $870912 \backslash \backslash \mathrm{tk} . \mathrm{MP} 2 \backslash \backslash 0,2 \backslash 0,-3.5514209977,3.0552011874,0.557094767 \backslash \mathrm{C},-2.6528$ $480478,2.4492426735,1.4714367541 \backslash \mathrm{C},-4.8782559967,2.527800642,0.7073294$ 
$025 \backslash \mathrm{C},-5.7613459502,3.2086837619,-0.2801926603 \backslash \mathrm{H},-4.862330906,1.438678$ $1689,0.5377795714 \backslash \mathrm{H},-5.2290983659,2.7031575186,1.7375479607 \backslash \mathrm{C},-5.65540$ $95361,2.7862279851,-1.7092360087 \backslash \mathrm{C},-6.1839937042,4.6096568304,0.020588$ $664 \backslash \mathrm{H},-7.0311412318,4.9148014418,-0.6018131367 \backslash \mathrm{H},-6.4667257253,4.72489$ $45871,1.071714774 \backslash \mathrm{H},-5.3608258348,5.3136890946,-0.1737300186 \backslash \mathrm{H},-6.5134$ $460671,3.1326124959,-2.2939839793 \backslash \mathrm{H},-4.749314182,3.2057303759,-2.17187$ $33517 \backslash \mathrm{H},-5.5905368121,1.6973819657,-1.8003646831 \backslash \mathrm{H},-1.6739359541,2.898$ $266135,1.2972867717 \backslash \mathrm{H},-2.9548939731,2.6308856486,2.512636828 \backslash \mathrm{H},-2.5872$ $827152,1.3636674876,1.3115803451 \backslash \backslash$ Version=ES64L-G09RevD .01 $\backslash$ State $=2-\mathrm{A} \backslash \mathrm{H}$ $\mathrm{F}=-270.547248 \backslash \mathrm{MP} 2=-271.3557084 \backslash \mathrm{PUHF}=-270.5505379 \backslash \mathrm{PMP} 2-0=-271.3578101 \backslash \mathrm{S}$ $2=0.762767 \backslash \mathrm{S} 2-1=0.75349 \backslash \mathrm{S} 2 \mathrm{~A}=0.750128 \backslash \mathrm{RMSD}=4.738 \mathrm{e}-09 \backslash \mathrm{RMSF}=7.288 \mathrm{e}-06 \backslash \mathrm{Dip}$ ole $=-0.0360142,-0.25515,0.2582003 \backslash P G=C 01[X(C 5 H 1101)] \backslash \backslash @$

Product 13-) HOOCH2

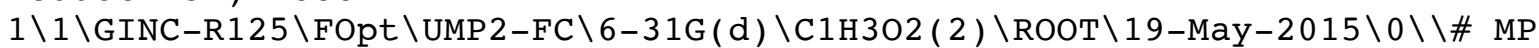
2/6-31G(d) SCF=Tight INT(grid=ultrafine) OPT IOP(2/17=4) maxdisk=13421 $7728 \backslash \backslash t \mathrm{k} . \mathrm{MP} 2 \backslash \backslash 0,2 \backslash \mathrm{O},-3.3439431727,2.3501979685,1.7846822351 \backslash \mathrm{H},-3.06378$ $69408,1.5987786631,2.344684232 \backslash 0,-3.9378532009,1.6026435765,0.67607390$ $86 \backslash \mathrm{C},-5.3017045116,1.664562547,0.8291051602 \backslash \mathrm{H},-5.7833416035,1.17967188$ $07,-0.011436408 \backslash \mathrm{H},-5.6971545706,2.5794343642,1.253980872 \backslash \backslash$ Version=ES 64 L-G09RevD . 01 \State $=2-A \backslash H F=-189.1619176 \backslash M P 2=-189.635925 \backslash P U H F=-189.16456$ $76 \backslash \mathrm{PMP} 2-0=-189.6375998 \backslash \mathrm{S} 2=0.759559 \backslash \mathrm{S} 2-1=0.752494 \backslash \mathrm{S} 2 \mathrm{~A}=0.750068 \backslash \mathrm{RMSD}=4.1$ $38 \mathrm{e}-09 \backslash \mathrm{RMSF}=1.248 \mathrm{e}-05 \backslash \mathrm{Dipole}=-0.2453271,-0.3464543,0.3579119 \backslash \mathrm{PG}=\mathrm{C} 01] \mathrm{X}$ ( C1H302) ] \\@

Product $14-)$ HOOC ( $\mathrm{CH} 3) 2$

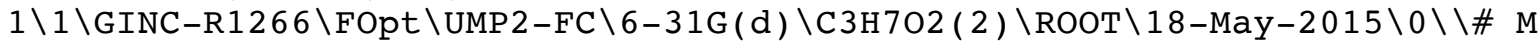
P2/6-31G(d) SCF=Tight INT(grid=ultrafine) OPT IOP(2/17=4) maxdisk=1342 $17728 \backslash \backslash \mathrm{tk} . \mathrm{MP} 2 \backslash \backslash 0,2 \backslash 0,-3.2964645314,2.3332781977,1.8232374845 \backslash \mathrm{H},-3.0371$ $17239,1.5320775942,2.3206343536 \backslash 0,-3.9364756237,1.6809942213,0.6738899$ $454 \backslash \mathrm{C},-5.318405925,1.691312873,0.8470076099 \backslash \mathrm{C},-5.9230384588,0.90333543$ $14,-0.2696167755 \backslash \mathrm{C},-5.9507794407,2.9652653117,1.3029423542 \backslash \mathrm{H},-7.019782$ $2014,2.8082123547,1.4666651197 \backslash \mathrm{H},-5.498169199,3.3244843152,2.227120108$ $5 \backslash \mathrm{H},-5.8325708796,3.7502412421,0.5410652401 \backslash \mathrm{H},-6.9777993615,0.71039933$ $74,-0.058079817 \backslash \mathrm{H},-5.8630212508,1.4458094349,-1.2241575599 \backslash \mathrm{H},-5.408453$ $8892,-0.0528943136,-0.3923100635 \backslash \backslash$ Version=ES64L-G09RevD.01\State $=2-\mathrm{A} \backslash \mathrm{H}$ $\mathrm{F}=-267.2447282 \backslash \mathrm{MP} 2=-267.9823436 \backslash \mathrm{PUHF}=-267.2475282 \backslash \mathrm{PMP} 2-0=-267.9841407 \backslash$ $\mathrm{S} 2=0.760873 \backslash \mathrm{S} 2-1=0.753043 \backslash \mathrm{S} 2 \mathrm{~A}=0.750096 \backslash \mathrm{RMSD}=8.732 \mathrm{e}-09 \backslash \mathrm{RMSF}=2.075 \mathrm{e}-05 \backslash \mathrm{D}$

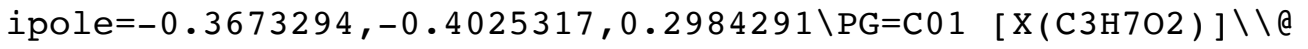

Product 15-) СH3OOCH2

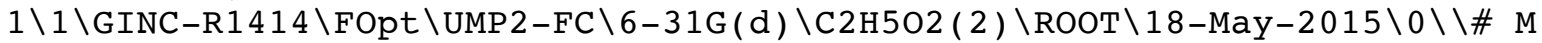
P2/6-31G(d) SCF=Tight INT(grid=ultrafine) OPT IOP(2/17=4) maxdisk=1342 $17728 \backslash \backslash t \mathrm{k} . \mathrm{MP} 2 \backslash \backslash 0,2 \backslash 0,-3.2186795403,2.130954199,0.9298562867 \backslash \mathrm{C},-2.52552$ $45235,3.0879522591,1.723850974 \backslash 0,-4.4046365179,1.801892717,1.743516114$ $8 \backslash \mathrm{C},-5.3984670757,1.4970230792,0.8520606749 \backslash \mathrm{H},-5.4171059676,2.07073233$ $29,-0.0669855513 \backslash \mathrm{H},-6.3069316288,1.2492819074,1.3862012346 \backslash \mathrm{H},-1.626950$ $7089,3.3077520982,1.1438952637 \backslash \mathrm{H},-3.1227708153,3.9938043968,1.86118460$ $91 \backslash \mathrm{H},-2.2524302219,2.6641030104,2.6940243935 \backslash \backslash$ Version=ES64L-G09RevD. 01 $\backslash$ State $=2-A \backslash H F=-228.1930977 \backslash M P 2=-228.7965936 \backslash \mathrm{PUHF}=-228.1957794 \backslash \mathrm{PMP} 2-0=-$ $228.7982924 \backslash \mathrm{S} 2=0.759774 \backslash \mathrm{S} 2-1=0.752579 \backslash \mathrm{S} 2 \mathrm{~A}=0.750069 \backslash \mathrm{RMSD}=9.595 e-09 \backslash \mathrm{RMSF}$ $=9.902 e-06 \backslash$ Dipole $=0.028522,0.5852546,0.1065262 \backslash P G=C 01[X(C 2 H 502)] \backslash \backslash @$

Product $16-) \mathrm{CH} 30 \mathrm{OC}(\mathrm{CH} 3) 2$

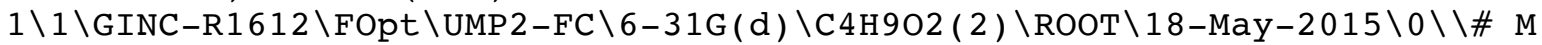
P2/6-31G(d) SCF=Tight INT(grid=ultrafine) OPT IOP(2/17=4) maxdisk=5368 $70912 \backslash \backslash \mathrm{tk} . \mathrm{MP} 2 \backslash \backslash 0,2 \backslash 0,-3.3995556693,3.3949548497,1.1705001926 \backslash \mathrm{C},-2.6359$ $645297,2.7352884941,2.1779042633 \backslash 0,-3.928683894,2.310095052,0.33077941$ $38 \backslash \mathrm{C},-5.2188499055,1.9956802366,0.747126496 \backslash \mathrm{C},-5.6671408425,0.78592166$ $64,-0.006574938 \backslash \mathrm{C},-6.1623348407,3.1271611908,0.9955050696 \backslash \mathrm{H},-2.2262161$ 
$616,3.5510626689,2.7784787841 \backslash \mathrm{H},-3.2730359347,2.0941723339,2.794859484$ $3 \backslash \mathrm{H},-1.8275623026,2.1497014154,1.7320625963 \backslash \mathrm{H},-7.1042856677,2.74213872$ $65,1.3942249539 \backslash \mathrm{H},-5.7435701432,3.8451800797,1.7007570499 \backslash \mathrm{H},-6.3802039$ $546,3.6624731604,0.059071056 \backslash \mathrm{H},-6.5831945197,0.389197719,0.4388103319 \backslash$ $\mathrm{H},-5.8781637462,1.0215143926,-1.0598185246 \backslash \mathrm{H},-4.8986488878,0.009349013$ $9,0.0176057711 \backslash \backslash$ Version=ES64L-G09RevD.01\State $=2-A \backslash H F=-306.2759465 \backslash M P 2$ $=-307.1439371 \backslash \mathrm{PUHF}=-306.2788011 \backslash \mathrm{PMP} 2-0=-307.1457829 \backslash \mathrm{S} 2=0.761315 \backslash \mathrm{S} 2-1=0$ $.753274 \backslash \mathrm{S} 2 \mathrm{~A}=0.750104 \backslash \mathrm{RMSD}=2.699 \mathrm{e}-09 \backslash \mathrm{RMSF}=1.098 \mathrm{e}-05 \backslash \mathrm{Dipole}=-0.1667375,-$ $0.3177518,0.5062312 \backslash \mathrm{PG}=\mathrm{C} 01[\mathrm{X}(\mathrm{C} 4 \mathrm{H} 9 \mathrm{O} 2)] \backslash \backslash @$

\section{Product 17-) HSCH $2 \mathrm{CH} 2$}

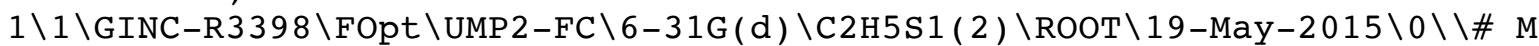
P2/6-31G(d) SCF=Tight INT(grid=ultrafine) OPT IOP(2/17=4) maxdisk=1342 $17728 \backslash \backslash t \mathrm{k} . \mathrm{MP} 2 \backslash \backslash 0,2 \backslash \mathrm{S},-2.971571903,2.6444019108,1.02992345 \backslash \mathrm{H},-2.4351183$ $474,1.4318535204,1.2326871579 \backslash \mathrm{C},-4.7310669448,2.0992459524,1.162330492$ $\backslash \mathrm{C},-5.0920240319,1.0670297674,0.1649273143 \backslash \mathrm{H},-5.301026197,3.0189424079$ $, 1.0059044701 \backslash \mathrm{H},-4.9173164101,1.7595047555,2.1827549386 \backslash \mathrm{H},-4.968778894$ $4,0.0167462429,0.3986521234 \backslash \mathrm{H},-5.2015912713,1.3403174427,-0.8766619463$

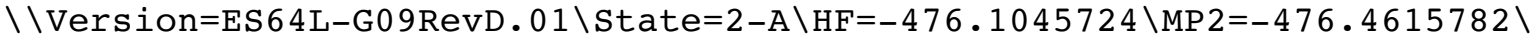
$\mathrm{PUHF}=-476.1081319 \backslash \mathrm{PMP} 2-0=-476.4639786 \backslash \mathrm{S} 2=0.76606 \backslash \mathrm{S} 2-1=0.755524 \backslash \mathrm{S} 2 \mathrm{~A}=0.7$ $50197 \backslash \mathrm{RMSD}=5.847 \mathrm{e}-09 \backslash \mathrm{RMSF}=2.963 e-05 \backslash \mathrm{Dipole}=-0.3576767,-0.5111512,0.083$ $8533 \backslash P G=C 01 \quad[X(C 2 H 5 S 1)] \backslash \backslash @$

\section{Product 18-) $\mathrm{HSCH} 2 \mathrm{C}(\mathrm{CH} 3) 2$}

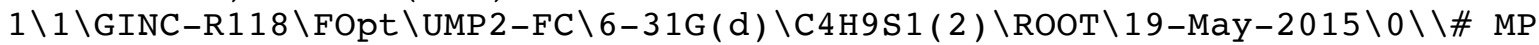
2/6-31G(d) SCF=Tight INT(grid=ultrafine) OPT IOP(2/17=4) maxdisk=13421 $7728 \backslash \backslash t \mathrm{k} . \mathrm{MP} 2 \backslash \backslash 0,2 \backslash \mathrm{S},-3.0073653331,2.3864577046,0.8877478387 \backslash \mathrm{H},-2.73154$ $57034,1.4158027414,1.7717158651 \backslash \mathrm{C},-4.8463716231,2.1502459273,0.9111868$ $723 \backslash \mathrm{C},-5.2880587685,0.8360799868,0.3834490681 \backslash \mathrm{H},-5.2212626958,2.970541$ $8785,0.291646725 \backslash \mathrm{H},-5.1951533686,2.3128379947,1.934689738 \backslash \mathrm{C},-5.2038431$ $329,-0.3574386243,1.2791676053 \backslash \mathrm{C},-5.2637885228,0.6169726391,-1.0940335$ $669 \backslash \mathrm{H},-5.8858672264,-1.1464252207,0.946791189 \backslash \mathrm{H},-4.1919847878,-0.79384$ $99452,1.2807454482 \backslash \mathrm{H},-5.4523881392,-0.1025849895,2.3145606107 \backslash \mathrm{H},-5.893$ $9109646,-0.2320937096,-1.376674354 \backslash \mathrm{H},-5.61380521,1.5018511805,-1.63515$ $18123 \backslash \mathrm{H},-4.2443955237,0.4023244365,-1.4488822272 \backslash \backslash$ Version=ES64L-G09ReV D. $01 \backslash$ State $=2-A \backslash H F=-554.1822487 \backslash M P 2=-554.8051087 \backslash P U H F=-554.1860248 \backslash P M P 2$ $-0=-554.8076365 \backslash \mathrm{S} 2=0.767189 \backslash \mathrm{S} 2-1=0.755728 \backslash \mathrm{S} 2 \mathrm{~A}=0.750244 \backslash \mathrm{RMSD}=4.197 \mathrm{e}-09 \backslash$ $\mathrm{RMSF}=1.352 \mathrm{e}-05 \backslash \mathrm{Dipole}=-0.4807873,-0.4704127,0.1751535 \backslash \mathrm{PG}=\mathrm{C} 01] \mathrm{X}(\mathrm{C} 4 \mathrm{H} 9 \mathrm{~S} 1$ )$] \backslash \backslash Q$

\section{Product 19-) $\mathrm{CH} 3 \mathrm{SCH} 2 \mathrm{CH} 2$}

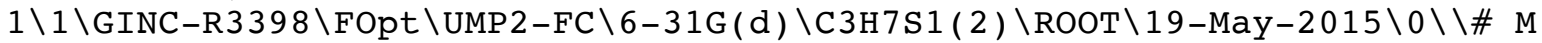
P2/6-31G(d) SCF=Tight INT(grid=ultrafine) OPT IOP(2/17=4) maxdisk=1342 $17728 \backslash \backslash t \mathrm{k} . M P 2 \backslash \backslash 0,2 \backslash \mathrm{S},-3.035390354,2.9755224183,0.1910978569 \backslash \mathrm{C},-2.59562$ $3164,2.9011836399,1.9431894069 \backslash \mathrm{C},-4.7880156181,2.4353303426,0.30596581$ $71 \backslash \mathrm{C},-4.9524743443,1.049315624,0.8033896074 \backslash \mathrm{H},-5.1630741101,2.53325774$ $16,-0.7178469207 \backslash \mathrm{H},-5.3299217225,3.1521508368,0.9298600742 \backslash \mathrm{H},-5.135122$ $4877,0.8523327466,1.8518950044 \backslash \mathrm{H},-4.6650970631,0.2149880724,0.17623672$ $49 \backslash \mathrm{H},-1.574396708,3.2757099763,2.0368780403 \backslash \mathrm{H},-3.2541956213,3.53709025$ $9,2.5405252121 \backslash \mathrm{H},-2.6281208071,1.8792053424,2.3242781765 \backslash \backslash$ Version=ES 64 L-G09RevD. 01 \State $=2-A \backslash H F=-515.1385017 \backslash M P 2=-515.6296302 \backslash P U H F=-515.1420$ $482 \backslash \mathrm{PMP} 2-0=-515.6320072 \backslash \mathrm{S} 2=0.765662 \backslash \mathrm{S} 2-1=0.755259 \backslash \mathrm{S} 2 \mathrm{~A}=0.750187 \backslash \mathrm{RMSD}=9$. $281 e-09 \backslash \mathrm{RMSF}=2.732 \mathrm{e}-05 \backslash \mathrm{Dipole}=-0.310413,-0.1750257,0.5575593 \backslash \mathrm{PG}=\mathrm{C} 01] \mathrm{X}$ ( C3H7S1) ] \\@

Product 20-) $\mathrm{CH} 3 \mathrm{SCH} 2 \mathrm{C}(\mathrm{CH} 3) 2$

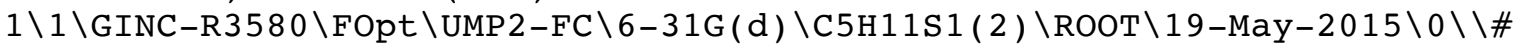
MP 2/6-31G(d) SCF=Tight INT(grid=ultrafine) OPT IOP $(2 / 17=4)$ maxdisk $=536$ $870912 \backslash \backslash \mathrm{tk} . \mathrm{MP} 2 \backslash \backslash 0,2 \backslash \mathrm{S},-3.4924274651,3.3368379177,0.3183019712 \backslash \mathrm{C},-2.689$ $7347335,2.5623602899,1.7412096681 \backslash \mathrm{C},-5.1801323896,2.6182302481,0.48817$ 
$12638 \backslash \mathrm{C},-5.2628659465,1.1447872133,0.3188237402 \backslash \mathrm{H},-5.7497969372,3.1288$ $663539,-0.2979288346 \backslash \mathrm{H},-5.5878733341,2.9325758148,1.4550941629 \backslash \mathrm{C},-5.25$ $12875305,0.2375032631,1.5063911336 \backslash \mathrm{C},-4.9532342,0.5594344903,-1.019876$ $8128 \backslash \mathrm{H},-1.7085404748,3.028827563,1.8525602848 \backslash \mathrm{H},-3.2610774512,2.737843$ $035,2.6564988342 \backslash \mathrm{H},-2.5493123352,1.4896716252,1.5991467386 \backslash \mathrm{H},-5.436840$ $0575,-0.4154282544,-1.1436391801 \backslash \mathrm{H},-5.2851451894,1.2163947335,-1.83005$ $17447 \backslash \mathrm{H},-3.8718304358,0.4069613691,-1.154475906 \backslash \mathrm{H},-5.911613743,-0.6228$ $62001,1.3436279015 \backslash \mathrm{H},-4.2496353278,-0.1746062147,1.7050315075 \backslash \mathrm{H},-5.581$ $1554488,0.7526115532,2.4133492718 \backslash \backslash$ Version=ES64L-G0 9RevD . $01 \backslash$ State=2-A $\mathrm{HF}=-593.214521 \backslash \mathrm{MP} 2=-593.9723363 \backslash \mathrm{PUHF}=-593.2184237 \backslash \mathrm{PMP} 2-0=-593.974928 \backslash \mathrm{S}$ $2=0.767294 \backslash S 2-1=0.755576 \backslash \mathrm{S} 2 \mathrm{~A}=0.750249 \backslash \mathrm{RMSD}=5.374 \mathrm{e}-09 \backslash \mathrm{RMSF}=2.708 \mathrm{e}-06 \backslash \mathrm{Di}$ pole $=-0.1874549,-0.5295287,0.4378965 \backslash \mathrm{PG}=\mathrm{C} 01[\mathrm{X}(\mathrm{C} 5 \mathrm{H} 11 \mathrm{~S} 1)] \backslash \backslash @$

Product 21-) $\mathrm{CH} 3 \mathrm{SCH} 2$

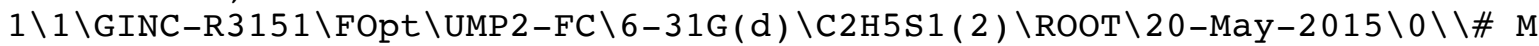
P2/6-31G(d) SCF=Tight INT(grid=ultrafine) OPT IOP(2/17=4) maxdisk=1342 $17728 \backslash \backslash \mathrm{tk} . \mathrm{MP} 2 \backslash \backslash 0,2 \backslash \mathrm{H},-2.7220188976,0.9847177183,0.312542441 \backslash \mathrm{C},-2.82787$ $59333,1.5722347835,1.2255306562 \backslash \mathrm{H},-2.4631124161,0.9875306198,2.0715279$ $81 \backslash \mathrm{H},-2.236043734,2.4866330134,1.1483431604 \backslash \mathrm{S},-4.5655764967,1.96009238$ $43,1.5401060983 \backslash \mathrm{C},-4.9238643171,2.9158567066,0.1566350214 \backslash \mathrm{H},-4.1340204$ $768,3.4600670386,-0.346070261 \backslash \mathrm{H},-5.9476007284,3.2454527356,0.045277902$ $8 \backslash \backslash$ Version=ES64L-G09RevD. $01 \backslash$ State $=2-A \backslash H F=-476.1075974 \backslash \mathrm{MP} 2=-476.4702569$ $\backslash \mathrm{PUHF}=-476.1111688 \backslash \mathrm{PMP} 2-0=-476.4725581 \backslash \mathrm{S} 2=0.765141 \backslash \mathrm{S} 2-1=0.754294 \backslash \mathrm{S} 2 \mathrm{~A}=0$ $.750179 \backslash \mathrm{RMSD}=7.617 \mathrm{e}-09 \backslash \mathrm{RMSF}=8.505 \mathrm{e}-06 \backslash \mathrm{Dipole}=0.5381107,0.089641,-0.202$ $529 \backslash \mathrm{PG}=\mathrm{C} 01 \quad[\mathrm{X}(\mathrm{C} 2 \mathrm{H} 5 \mathrm{~S} 1)] \backslash \backslash @$

Product 22-) $\mathrm{CH} 3 \mathrm{SC}(\mathrm{CH} 3) 2$

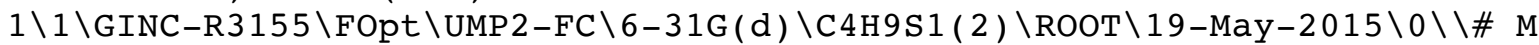
P2/6-31G(d) SCF=Tight INT(grid=ultrafine) OPT IOP(2/17=4) maxdisk=2684 $35456 \backslash \backslash \mathrm{tk} . \mathrm{MP} 2 \backslash \backslash 0,2 \backslash \mathrm{H},-3.3907023924,2.78438083,-0.1506250168 \backslash \mathrm{C},-3.01084$ $89715,2.2941472447,0.7467432949 \backslash \mathrm{H},-2.1978639717,1.6144954035,0.4854029$ $613 \backslash \mathrm{H},-2.6236119811,3.0587752807,1.4230333799 \backslash \mathrm{S},-4.3122133832,1.421448$ $7703,1.6514137366 \backslash \mathrm{C},-4.6871318819,0.0799966647,0.5958473218 \backslash \mathrm{C},-5.63081$ $80597,-0.9183216792,1.1937824268 \backslash \mathrm{C},-4.7031060063,0.252838103,-0.890708$ $744 \backslash \mathrm{H},-4.7747910575,-0.7266556752,-1.3737960797 \backslash \mathrm{H},-5.5698636183,0.8474$ $393407,-1.2197302707 \backslash \mathrm{H},-3.8038958523,0.7445508634,-1.2698574196 \backslash \mathrm{H},-6.6$ $811530979,-0.6066372761,1.0894787784 \backslash \mathrm{H},-5.5268492024,-1.8819250905,0.6$ $839812677 \backslash \mathrm{H},-5.4324735239,-1.0725687801,2.2580563633 \backslash \backslash$ Version=ES64L-G0 9RevD.01 \State $=2-\mathrm{A} \backslash \mathrm{HF}=-554.1829222 \backslash \mathrm{MP} 2=-554.8103212 \backslash \mathrm{PUHF}=-554.1865311 \backslash$ $\mathrm{PMP} 2-0=-554.8126777 \backslash \mathrm{S} 2=0.76578 \backslash \mathrm{S} 2-1=0.754742 \backslash \mathrm{S} 2 \mathrm{~A}=0.750207 \backslash \mathrm{RMSD}=7.431 \mathrm{e}-$ $09 \backslash \mathrm{RMSF}=1.697 \mathrm{e}-05 \backslash \mathrm{Dipole}=0.1990046,0.0300177,-0.5445609 \backslash \mathrm{PG}=\mathrm{C} 01]$ [X(C4H9 $\mathrm{S} 1)] \backslash \backslash$ a

Product 23-) СH3CH2SCH2

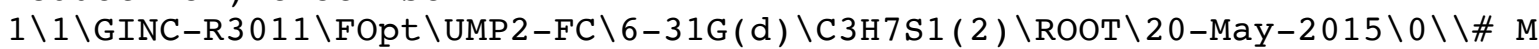
P2/6-31G(d) SCF=Tight INT(grid=ultrafine) OPT IOP(2/17=4) maxdisk=1342 $17728 \backslash \backslash \mathrm{tk} . \mathrm{MP} 2 \backslash \backslash 0,2 \backslash \mathrm{H},-3.206146125,1.3285546347,0.1342700741 \backslash \mathrm{C},-3.12544$ $63893,1.6733984623,1.170092033 \backslash \mathrm{H},-2.9382115906,0.7933071509,1.79200748$ $75 \backslash \mathrm{C},-2.0059452889,2.6943354895,1.3257625947 \backslash \mathrm{S},-4.7499626543,2.2948051$ $132,1.6930644808 \backslash \mathrm{C},-5.0467843441,3.4638670422,0.4674924904 \backslash \mathrm{H},-4.629009$ $3824,3.3270468313,-0.5230470469 \backslash \mathrm{H},-5.9255966948,4.0826292247,0.5872909$ $312 \backslash \mathrm{H},-2.2005897804,3.581186149,0.7189370376 \backslash \mathrm{H},-1.9145760004,3.0097657$ $353,2.3676731593 \backslash \mathrm{H},-1.0525807498,2.2600891669,1.0086627583 \backslash \backslash$ Version=ES

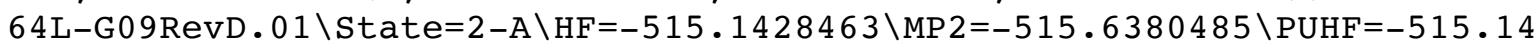
$6427 \backslash \mathrm{PMP} 2-0=-515.6403639 \backslash \mathrm{S} 2=0.765384 \backslash \mathrm{S} 2-1=0.754434 \backslash \mathrm{S} 2 \mathrm{~A}=0.750184 \backslash \mathrm{RMSD}=5$ $.516 e-09 \backslash \mathrm{RMSF}=1.629 \mathrm{e}-05 \backslash \mathrm{Dipole}=0.4509709,-0.1128035,-0.3660555 \backslash \mathrm{PG}=\mathrm{C} 01$ $[\mathrm{X}(\mathrm{C} 3 \mathrm{H} 7 \mathrm{~S} 1)] \backslash \backslash @$

Product 24-) $\mathrm{CH} 3 \mathrm{CH} 2 \mathrm{SC}(\mathrm{CH} 3) 2$

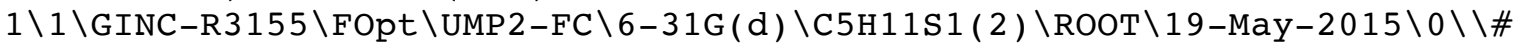


MP2/6-31G(d) SCF=Tight INT(grid=ultrafine) OPT IOP(2/17=4) maxdisk=268 $435456 \backslash \backslash \mathrm{tk}$.MP $2 \backslash \backslash 0,2 \backslash \mathrm{H},-3.1017528678,1.2221919324,0.1483967716 \backslash \mathrm{C},-3.094$ $6475148,1.6327669464,1.1615960828 \backslash \mathrm{H},-2.9171558647,0.7942886016,1.84214$ $56614 \backslash \mathrm{C},-2.0139154327,2.6928730979,1.3261137449 \backslash \mathrm{S},-4.7557902721,2.2245$ $420231,1.6022625949 \backslash \mathrm{C},-5.0304388864,3.5119298575,0.4514546457 \backslash \mathrm{C},-4.718$ $7745104,3.3230213041,-1.0009329367 \backslash \mathrm{C},-6.1650352732,4.4247041867,0.8031$ $873041 \backslash \mathrm{H},-6.0513117831,5.3804861032,0.2803054852 \backslash \mathrm{H},-7.1383222908,4.006$ $6163555,0.5040805976 \backslash \mathrm{H},-6.2022721596,4.6231283627,1.8775368531 \backslash \mathrm{H},-5.46$ $88666803,2.6823262287,-1.4899107604 \backslash \mathrm{H},-4.7311963559,4.2914308921,-1.51$ $16067583 \backslash \mathrm{H},-3.7394947965,2.869550089,-1.1722946447 \backslash \mathrm{H},-2.2038780334,3.5$ $510257225,0.6776612061 \backslash \mathrm{H},-1.9847695117,3.0511564917,2.357790047 \backslash \mathrm{H},-1.0$ $329477665,2.2777188051,1.0731751057 \backslash \backslash$ Version=ES64L-G09RevD.01\State=2$\mathrm{A} \backslash \mathrm{HF}=-593.2177642 \backslash \mathrm{MP} 2=-593.9783614 \backslash \mathrm{PUHF}=-593.221411 \backslash \mathrm{PMP} 2-0=-593.980759$ $5 \backslash S 2=0.766289 \backslash S 2-1=0.755044 \backslash S 2 A=0.75022 \backslash R M S D=7.980 e-09 \backslash R M S F=6.818 e-06 \backslash$ Dipole $=0.364937,0.0676154,-0.4395927 \backslash \mathrm{PG}=\mathrm{C} 01 \quad[\mathrm{X}(\mathrm{C} 5 \mathrm{H} 11 \mathrm{~S} 1)] \backslash \backslash @$

Product 25-) HSSCH2

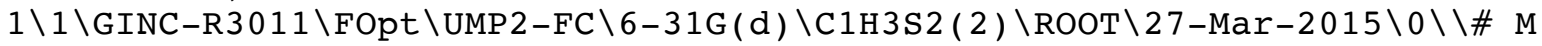
P2/6-31G(d) SCF=Tight INT(grid=ultrafine) OPT IOP(2/17=4) maxdisk=1342 $17728 \backslash \backslash \mathrm{hstk} . \mathrm{MP} 2 \backslash \backslash 0,2 \backslash \mathrm{S}, 0.1581396749,0.349390264,-0.8292021762 \backslash \mathrm{C}, 1.2986$ $267398,1.2453964599,0.1023901215 \backslash \mathrm{S},-1.2741818049,1.7711802237,-1.30642$ $84606 \backslash \mathrm{H},-2.1338845383,1.5310756763,-0.3010248738 \backslash \mathrm{H}, 0.9840161128,2.0678$ $399776,0.7291532748 \backslash \mathrm{H}, 2.2874098157,0.8174313986,0.1962621141 \backslash \backslash$ Version= ES64L-G09RevD. 01 \State $=2-A \backslash H F=-834.5811955 \backslash M P 2=-834.9284385 \backslash P U H F=-834$. $5849949 \backslash \mathrm{PMP} 2-0=-834.9310472 \backslash \mathrm{S} 2=0.76856 \backslash \mathrm{S} 2-1=0.756828 \backslash \mathrm{S} 2 \mathrm{~A}=0.750268 \backslash \mathrm{RMSD}$ $=4.558 e-09 \backslash \mathrm{RMSF}=8.033 e-06 \backslash \mathrm{Dipole}=-0.0301291,0.1003587,0.6135329 \backslash \mathrm{PG}=\mathrm{C} 01$

$[\mathrm{X}(\mathrm{C} 1 \mathrm{H} 3 \mathrm{~S} 2)] \backslash \backslash @$

Product 26-) HSSC ( $\mathrm{CH} 3) 2$

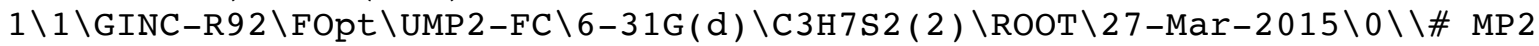
/6-31G(d) SCF=Tight INT(grid=ultrafine) OPT IOP(2/17=4) maxdisk=134217 $728 \backslash \backslash \mathrm{HSSCCH} 32$.MP $2 \backslash \backslash 0,2 \backslash \mathrm{S}, 0.2654537651,0.049737918,-0.5040001305 \backslash \mathrm{C}, 1.43$ $7464361,1.0894606643,0.2532888275 \backslash S,-1.3107021064,1.3131542462,-1.0732$ $852273 \backslash \mathrm{H},-2.0685631161,1.2172735941,0.0332074774 \backslash \mathrm{C}, 1.0265267664,1.9906$ $253228,1.3700973054 \backslash \mathrm{C}, 2.7407145399,1.3210661633,-0.441778196 \backslash \mathrm{H}, 3.54012$ $02359,1.4951464818,0.2859316396 \backslash \mathrm{H}, 3.0173700255,0.4743136808,-1.0736466$ $416 \backslash \mathrm{H}, 2.6838106527,2.2115340147,-1.0865632892 \backslash \mathrm{H}, 1.8971132134,2.2736992$ $563,1.9698959225 \backslash \mathrm{H}, 0.5744207397,2.9168935804,0.9873383015 \backslash \mathrm{H}, 0.29384892$ $3,1.5085710775,2.0232110107 \backslash \backslash$ Version=ES64L-G09RevD . 01 \State=2-A \HF=-91 $2.6591252 \backslash M P 2=-913.2717799 \backslash P U H F=-912.6637387 \backslash P M P 2-0=-913.2751827 \backslash S 2=0$. $779929 \backslash \mathrm{S} 2-1=0.764021 \backslash \mathrm{S} 2 \mathrm{~A}=0.750642 \backslash \mathrm{RMSD}=6.409 \mathrm{e}-09 \backslash \mathrm{RMSF}=7.058 \mathrm{e}-06 \backslash \mathrm{Dipole}$ $=0.3193097,0.4614651,0.6409855 \backslash P G=C 01[X(C 3 H 7 S 2)] \backslash \backslash @$

Product 27-) $\mathrm{CH} 3 \mathrm{SSCH} 2$

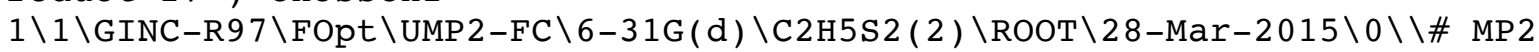
/6-31G(d) SCF=Tight INT(grid=ultrafine) OPT IOP(2/17=4) maxdisk=671088 $640 \backslash \backslash \mathrm{CH} 3 \mathrm{SSCH} 2$. MP2 $\backslash \backslash 0,2 \backslash \mathrm{S}, 0.3991817155,0.327933743,-0.9226203938 \backslash \mathrm{C}, 1.36$ $76825536,1.1853775959,0.2212942761 \backslash \mathrm{S},-1.1044965677,1.6801667803,-1.327$ $5569819 \backslash \mathrm{C},-2.1674842603,1.4733030981,0.1274513621 \backslash \mathrm{H}, 1.4422066937,2.263$ $6372547,0.1645314528 \backslash \mathrm{H}, 2.1186678596,0.6080542547,0.7450335521 \backslash \mathrm{H},-3.006$ $2319904,2.1632099756,0.0033478087 \backslash \mathrm{H},-2.5468831624,0.452737899,0.190668$ $1228 \backslash \mathrm{H},-1.6259018416,1.7272123987,1.0395398012 \backslash \backslash$ Version=ES64L-G09RevD. $01 \backslash$ State $=2-A \backslash H F=-873.6186927 \backslash M P 2=-874.1007322 \backslash P U H F=-873.6223014 \backslash P M P 2-0$ $=-874.1031436 \backslash \mathrm{S} 2=0.766516 \backslash \mathrm{S} 2-1=0.755472 \backslash \mathrm{S} 2 \mathrm{~A}=0.750212 \backslash \mathrm{RMSD}=7.061 \mathrm{e}-09 \backslash \mathrm{RM}$ $\mathrm{SF}=6.652 \mathrm{e}-06 \backslash \mathrm{Dipole}=-0.1600962,0.1615902,0.6779372 \backslash \mathrm{PG}=\mathrm{C} 01 \quad[\mathrm{X}(\mathrm{C} 2 \mathrm{H} 5 \mathrm{~S} 2)] \backslash$ $\backslash$ Q

Product 28-) $\mathrm{CH} 3 \mathrm{SSC}(\mathrm{CH} 3) 2$

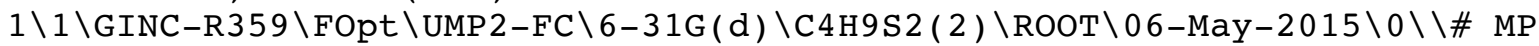
2/6-31G(d) SCF=Tight INT(grid=ultrafine) OPT IOP(2/17=4) maxdisk=93952 
$4096 \backslash \backslash t \mathrm{tk} . \mathrm{MP} 2 \backslash \backslash 0,2 \backslash \mathrm{S}, 0.394508727,0.3018096436,-0.9913997443 \backslash \mathrm{C}, 1.5417221$ $673,1.1444416857,0.0166680249 \backslash S,-1.3522872409,1.4403392655,-0.93469914$ $04 \backslash \mathrm{C},-2.28287974,0.6589015327,0.4117299728 \backslash \mathrm{C}, 1.166480786,1.5341882839$, $1.4083822566 \backslash \mathrm{C}, 2.7301228622,1.7738838836,-0.636783559 \backslash \mathrm{H}, 3.5834375267,1$ $.791433981,0.0492561791 \backslash \mathrm{H}, 3.0197199343,1.2407364007,-1.5451061698 \backslash \mathrm{H}, 2$. $5137331472,2.8156093999,-0.9197333613 \backslash \mathrm{H}, 2.0575276155,1.8063836169,1.98$ $22552605 \backslash \mathrm{H}, 0.4928145575,2.4035404436,1.410729062 \backslash \mathrm{H}, 0.6558025993,0.7161$ $480626,1.924834495 \backslash \mathrm{H},-3.2779072083,1.1123187112,0.4050125439 \backslash \mathrm{H},-2.3806$ $26347,-0.4141164762,0.2413320034 \backslash \mathrm{H},-1.8175423868,0.8387895652,1.381501$ $1765 \backslash \backslash$ Version=ES64L-G09RevD.01 \State $=2-\mathrm{A} \backslash \mathrm{HF}=-951.694676 \backslash \mathrm{MP} 2=-952.44245$ $69 \backslash \mathrm{PUHF}=-951.6991424 \backslash \mathrm{PMP} 2-0=-952.4456996 \backslash \mathrm{S} 2=0.777229 \backslash \mathrm{S} 2-1=0.762125 \backslash \mathrm{S} 2 \mathrm{~A}$ $=0.750561 \backslash \mathrm{RMSD}=9.609 \mathrm{e}-09 \backslash \mathrm{RMSF}=2.391 \mathrm{e}-06 \backslash \mathrm{Dipole}=0.109883,0.1635548,0.84$ $02322 \backslash \mathrm{PG}=\mathrm{C} 01 \quad[\mathrm{X}(\mathrm{C} 4 \mathrm{H} 9 \mathrm{~S} 2)] \backslash \backslash @$

\section{RSE Products}

Product $1-\mathrm{H}$

$\mathrm{CH} 3 \mathrm{CH} 2 \mathrm{CH} 2-\mathrm{H}$

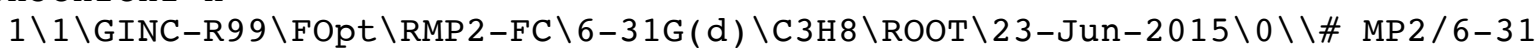
G(d) SCF=Tight INT(grid=ultrafine) OPT IOP $(2 / 17=4)$ maxdisk=134217728\\} diMetk.MP $2 \backslash \backslash 0,1 \backslash \mathrm{C},-2.7593268019,1.2235683519,0.0072006845 \backslash \mathrm{C},-1.5280053$ $035,2.1249629646,-0.0139568017 \backslash C,-0.225038877,1.3304528318,-0.00192933$ $56 \backslash \mathrm{H},-3.6844060407,1.8073774874,-0.0017268186 \backslash \mathrm{H},-2.7661408487,0.595633$ $7751,0.9037035715 \backslash \mathrm{H},-2.7710411697,0.5608399123,-0.8638443656 \backslash \mathrm{H},-1.5533$ $073524,2.7987033122,0.8501934808 \backslash \mathrm{H},-1.5581700913,2.7641762947,-0.90379$ $85654 \backslash \mathrm{H},-0.1589292908,0.7055938347,0.8943108369 \backslash \mathrm{H}, 0.6475104474,1.99007$ $75807,-0.0173329714 \backslash \mathrm{H},-0.1638296117,0.6707999719,-0.8732371002 \backslash \backslash$ Versio $\mathrm{n}=\mathrm{ES} 64 \mathrm{~L}-\mathrm{G} 09$ RevD. $01 \backslash$ State $=1-\mathrm{A} 1 \backslash \mathrm{HF}=-118.2632604 \backslash \mathrm{MP} 2=-118.6603432 \backslash \mathrm{RMSD}=5$. $078 \mathrm{e}-09 \backslash \mathrm{RMSF}=2.083 e-05 \backslash \mathrm{Dipole}=-0.0008683,0.0205529,-0.0004022 \backslash \mathrm{PG}=\mathrm{C} 02 \mathrm{~V}$ $\left[\mathrm{C} 2(\mathrm{C} 1), \mathrm{SGV}(\mathrm{C} 2 \mathrm{H} 2), \mathrm{SGV}^{\prime}(\mathrm{H} 2), \mathrm{X}(\mathrm{H} 4)\right] \backslash \backslash @$

\section{Product $1-\mathrm{CH} 3$}

\section{$\mathrm{CH} 3 \mathrm{CH} 2 \mathrm{CH} 2-\mathrm{CH} 3$}

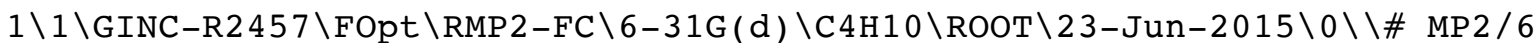
$-31 \mathrm{G}(\mathrm{d})$ SCF=Tight INT(grid=ultrafine) OPT IOP(2/17=4) maxdisk=40265318 $4 \backslash \backslash$ diMetk.MP $2 \backslash \backslash 0,1 \backslash H,-2.4461476407,2.3177811167,0.2756719915 \backslash \mathrm{C},-3.0008$ $022334,2.1359123102,1.2015292997 \backslash \mathrm{H},-2.3838840226,1.4957818305,1.839945$ $0146 \backslash \mathrm{H},-3.1282127054,3.0956425901,1.7109228839 \backslash \mathrm{C},-4.3453114039,1.47562$ $77097,0.9080770328 \backslash \mathrm{C},-4.2037717191,0.1323171547,0.1965560427 \backslash \mathrm{H},-4.9584$ $930186,2.1453751493,0.2919538358 \backslash \mathrm{H},-4.8966579305,1.3290332822,1.845460$ $7327 \backslash \mathrm{H},-3.5905901043,-0.5374302849,0.8126792398 \backslash \mathrm{H},-3.6524251925,0.2789$ $115822,-0.7408276572 \backslash \mathrm{C},-5.5482808896,-0.5279674458,-0.0968962242 \backslash \mathrm{H},-6$. $1029354822,-0.7098362522,0.828961084 \backslash \mathrm{H},-6.1651991004,0.112163034,-0.73$ $53119391 \backslash \mathrm{H},-5.4208704176,-1.4876977257,-0.6062898084 \backslash \backslash$ Version=ES64L-G0 9RevD.01 \State $=1-\mathrm{AG} \backslash \mathrm{HF}=-157.2978828 \backslash \mathrm{MP} 2=-157.8260366 \backslash \mathrm{RMSD}=7.068 \mathrm{e}-09 \backslash \mathrm{RM}$ $\mathrm{SF}=4.013 e-05 \backslash \mathrm{Dipole}=0 ., 0 ., 0 . \backslash \mathrm{PG}=\mathrm{C} 02 \mathrm{H} \quad[\mathrm{SGH}(\mathrm{C} 4 \mathrm{H} 2), \mathrm{X}(\mathrm{H} 8)] \backslash \backslash @$

\section{Product $2-\mathrm{H}$}

$\mathrm{CH} 3 \mathrm{CH} 2 \mathrm{C}(\mathrm{CH} 3) 2-\mathrm{H}$

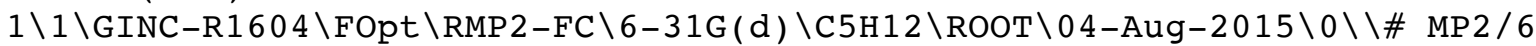
$-31 \mathrm{G}(\mathrm{d})$ SCF=Tight INT (grid=ultrafine) OPT IOP(2/17=4) maxdisk=40265318 $4 \backslash \backslash$ diMetk.MP2 $\backslash \backslash 0,1 \backslash \mathrm{H},-2.4140402005,1.6982358169,-0.0539842472 \backslash \mathrm{C},-2.784$ $1762932,1.7808015502,0.9709354227 \backslash \mathrm{H},-2.5089507823,0.8644117348,1.50326$ $03925 \backslash \mathrm{H},-2.2568540219,2.6137709548,1.4453766034 \backslash \mathrm{C},-4.2951758561,1.9985$ $449149,1.0134434707 \backslash \mathrm{C},-5.123860556,0.8232555776,0.4855365526 \backslash \mathrm{H},-4.5557$ $014222,2.8975997126,0.4379294088 \backslash \mathrm{H},-4.5978408126,2.199256711,2.0492043$ $154 \backslash \mathrm{H},-4.8509493612,-0.0665606271,1.0718749037 \backslash \mathrm{C},-4.8364832204,0.52894$ $22015,-0.9861352198 \backslash \mathrm{H},-5.4645786096,-0.2915371258,-1.3477248573 \backslash \mathrm{H},-5.0$ $522102621,1.411633251,-1.5993624707 \backslash \mathrm{H},-3.7937675318,0.2469203437,-1.15$ $38150271 \backslash \mathrm{C},-6.6144729843,1.0883165897,0.6930046235 \backslash \mathrm{H},-7.2187879064,0.2$ 
$391541498,0.3581178663 \backslash \mathrm{H},-6.841915778,1.2712863106,1.7480988481 \backslash \mathrm{H},-6.9$ $285344014,1.9697609336,0.1227034144 \backslash \backslash$ Version=ES64L-G09RevD. $01 \backslash$ State=1$\mathrm{A} \backslash \mathrm{HF}=-196.3310466 \backslash \mathrm{MP} 2=-196.993365 \backslash \mathrm{RMSD}=3.239 \mathrm{e}-09 \backslash \mathrm{RMSF}=3.052 \mathrm{e}-06 \backslash \mathrm{Dipole}$ $=0.0098545,-0.0096227,0.018809 \backslash \mathrm{PG}=\mathrm{C} 01 \quad[\mathrm{X}(\mathrm{C} 5 \mathrm{H} 12)] \backslash \backslash \mathrm{a}$

Product $2-\mathrm{CH} 3$

$\mathrm{CH} 3 \mathrm{CH} 2 \mathrm{C}(\mathrm{CH} 3) 2-\mathrm{CH} 3$

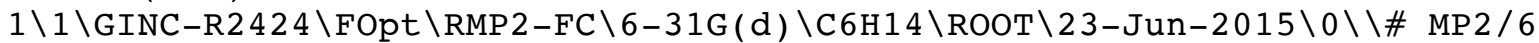
$-31 \mathrm{G}(\mathrm{d}) \mathrm{SCF}=\mathrm{Tight}$ INT(grid=ultrafine) OPT IOP(2/17=4) maxdisk=40265318

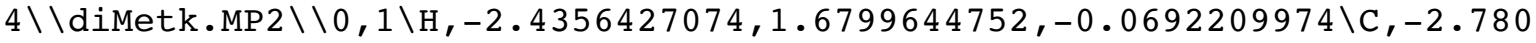
$5062042,1.80541528,0.9604833597 \backslash \mathrm{H},-2.4238035248,0.9524414912,1.5437146$ $284 \backslash \mathrm{H},-2.2969126694,2.7015502626,1.3611400469 \backslash \mathrm{C},-4.2999551504,1.949540$ $0494,1.0366445615 \backslash \mathrm{C},-5.1293957968,0.7653385228,0.508592533 \backslash \mathrm{H},-4.600148$ $612,2.8486211972,0.4811558974 \backslash \mathrm{H},-4.5883992116,2.1266153648,2.081859868$ $1 \backslash \mathrm{C},-4.827381651,-0.5072373018,1.3035491713 \backslash \mathrm{C},-4.8441071819,0.52055394$ $38,-0.9750882772 \backslash \mathrm{H},-5.4780001988,-1.3255574372,0.975956054 \backslash \mathrm{H},-3.791894$ $6185,-0.8335044679,1.168655296 \backslash \mathrm{H},-4.9984782891,-0.3485957874,2.3744075$ $3 \backslash \mathrm{H},-5.4951738677,-0.2702279144,-1.3637344049 \backslash \mathrm{H},-5.0273779417,1.427300$ $5965,-1.5627966662 \backslash \mathrm{H},-3.808862538,0.2091816596,-1.1430044572 \backslash \mathrm{C},-6.6112$ $263134,1.1130780628,0.676319109 \backslash \mathrm{H},-7.2471045802,0.2980445761,0.3133614$ $645 \backslash \mathrm{H},-6.8545584486,1.2889978869,1.7300456143 \backslash \mathrm{H},-6.8664220648,2.018022$ $3262,0.1137812265 \backslash \backslash$ Version=ES64L-G09RevD.01 \State $=1-A^{\prime} \backslash H F=-235.3635871$ $\backslash \mathrm{MP} 2=-236.1625388 \backslash \mathrm{RMSD}=4.521 \mathrm{e}-09 \backslash \mathrm{RMSF}=1.567 \mathrm{e}-05 \backslash \mathrm{Dipole}=0.0120342,0.009$ $6202,0.0042509 \backslash \mathrm{PG}=\mathrm{CS} \quad[\mathrm{SG}(\mathrm{C} 4 \mathrm{H} 2), \mathrm{X}(\mathrm{C} 2 \mathrm{H} 12)] \backslash \backslash \mathrm{a}$

Product $3-\mathrm{H}$

$\mathrm{CH} 3 \mathrm{CH} 2 \mathrm{CH} 2 \mathrm{CH} 2-\mathrm{H}$

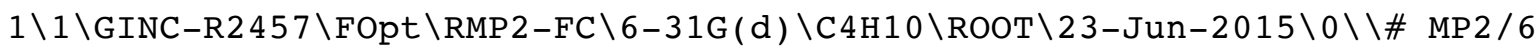
$-31 \mathrm{G}(\mathrm{d}) \mathrm{SCF}=\mathrm{Tight}$ INT(grid=ultrafine) OPT IOP(2/17=4) maxdisk=40265318 $4 \backslash \backslash$ diMetk. MP2 $\backslash \backslash 0,1 \backslash \mathrm{H},-2.4461476407,2.3177811167,0.2756719915 \backslash \mathrm{C},-3.0008$ $022334,2.1359123102,1.2015292997 \backslash \mathrm{H},-2.3838840226,1.4957818305,1.839945$ $0146 \backslash \mathrm{H},-3.1282127054,3.0956425901,1.7109228839 \backslash \mathrm{C},-4.3453114039,1.47562$ $77097,0.9080770328 \backslash \mathrm{C},-4.2037717191,0.1323171547,0.1965560427 \backslash \mathrm{H},-4.9584$ $930186,2.1453751493,0.2919538358 \backslash \mathrm{H},-4.8966579305,1.3290332822,1.845460$ $7327 \backslash \mathrm{H},-3.5905901043,-0.5374302849,0.8126792398 \backslash \mathrm{H},-3.6524251925,0.2789$ $115822,-0.7408276572 \backslash \mathrm{C},-5.5482808896,-0.5279674458,-0.0968962242 \backslash \mathrm{H},-6$. $1029354822,-0.7098362522,0.828961084 \backslash \mathrm{H},-6.1651991004,0.112163034,-0.73$ $53119391 \backslash \mathrm{H},-5.4208704176,-1.4876977257,-0.6062898084 \backslash \backslash$ Version=ES64L-G0 9RevD.01 \State $=1-A G \backslash H F=-157.2978828 \backslash M P 2=-157.8260366 \backslash$ RMSD $=7.068 e-09 \backslash R M$ $\mathrm{SF}=4.013 \mathrm{e}-05 \backslash \mathrm{Dipole}=0 ., 0 ., 0 . \backslash \mathrm{PG}=\mathrm{C} 02 \mathrm{H} \quad[\mathrm{SGH}(\mathrm{C} 4 \mathrm{H} 2), \mathrm{X}(\mathrm{H} 8)] \backslash \backslash \mathrm{Q}$

Product $3-\mathrm{CH} 3$

$\mathrm{CH} 3 \mathrm{CH} 2 \mathrm{CH} 2 \mathrm{CH} 2-\mathrm{CH} 3$

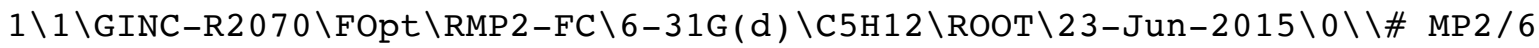
$-31 \mathrm{G}(\mathrm{d}) \mathrm{SCF}=\mathrm{Tight}$ INT(grid=ultrafine) OPT IOP(2/17=4) maxdisk=40265318 $4 \backslash \backslash$ diMetk. MP $2 \backslash \backslash 0,1 \backslash \mathrm{H},-2.3854394818,1.5908036386,0.1467058335 \backslash \mathrm{C},-2.8091$ $564272,1.8763572116,1.1177439528 \backslash \mathrm{H},-2.6341544113,1.0282744664,1.791630$ $147 \backslash \mathrm{C},-2.0857255013,3.1109743536,1.6493442063 \backslash \mathrm{C},-4.3129957341,2.091651$ $4326,0.9639765775 \backslash \mathrm{C},-5.0492387083,0.8638865524,0.432875504 \backslash \mathrm{H},-4.490269$ $3127,2.9403274409,0.2891831014 \backslash \mathrm{H},-4.739182787,2.3774641124,1.935418203$ $1 \backslash \mathrm{H},-4.8708164689,0.017435719,1.1079150683 \backslash \mathrm{H},-4.6220116322,0.579795868$ $3,-0.5370534416 \backslash \mathrm{H},-2.2234852442,3.9627182977,0.9759428913 \backslash \mathrm{H},-2.4738056$ $784,3.3962666396,2.6321544261 \backslash \mathrm{H},-1.0109946002,2.9332099798,1.750979618$ $1 \backslash \mathrm{C},-6.5511285332,1.0927498247,0.2839487504 \backslash \mathrm{H},-7.0567732832,0.20064854$ $8,-0.0974370625 \backslash \mathrm{H},-7.002984257,1.3494660558,1.2471922325 \backslash \mathrm{H},-6.75248174$ $39,1.9155754108,-0.4091088083 \backslash \backslash$ Version=ES64L-G09RevD . $01 \backslash$ State $=1-A \backslash H F=-$ $196.3324416 \backslash M P 2=-196.9917256 \backslash \mathrm{RMSD}=6.204 \mathrm{e}-09 \backslash \mathrm{RMSF}=3.641 \mathrm{e}-05 \backslash \mathrm{Dipole}=0.00$ $90262,-0.0169689,-0.0044371 \backslash \mathrm{PG}=\mathrm{C} 02 \quad[\mathrm{C} 2(\mathrm{C} 1), \mathrm{X}(\mathrm{C} 4 \mathrm{H} 12)] \backslash \backslash @$

Product $4-\mathrm{H}$ 
$\mathrm{CH} 3 \mathrm{CH} 2 \mathrm{CH} 2 \mathrm{C}(\mathrm{CH} 3) 2-\mathrm{H}$

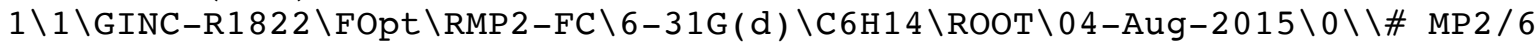
$-31 \mathrm{G}(\mathrm{d}) \quad \mathrm{SCF}=\mathrm{Tight}$ INT (grid=ultrafine) OPT IOP(2/17=4) maxdisk=40265318 $4 \backslash \backslash$ diMetk.MP2 $\backslash \backslash 0,1 \backslash \mathrm{H},-2.6092513754,1.4046668661,0.0933052603 \backslash \mathrm{C},-3.0323$ $786484,1.6624296324,1.0702407638 \backslash \mathrm{H},-2.9826983469,0.7514609787,1.680729$ $4663 \backslash \mathrm{C},-2.1816381398,2.755866691,1.712393066 \backslash \mathrm{C},-4.4932317757,2.0814149$ $593,0.9141838018 \backslash \mathrm{C},-5.4321693105,0.9879753859,0.3950926708 \backslash \mathrm{H},-4.552609$ $6884,2.9483951088,0.2396576248 \backslash \mathrm{H},-4.8639178924,2.426867054,1.889450753$ $2 \backslash \mathrm{H},-5.3582417702,0.1312079896,1.0809157546 \backslash \mathrm{C},-5.0464286255,0.51114138$ $89,-1.0046370488 \backslash \mathrm{H},-2.2035378984,3.6694040933,1.1099229176 \backslash \mathrm{H},-2.555267$ $6174,3.0055165249,2.710357874 \backslash \mathrm{H},-1.1375696283,2.4444916564,1.812185042$ $9 \backslash \mathrm{H},-5.7547608288,-0.2407223756,-1.3674615868 \backslash \mathrm{H},-5.0590376073,1.351097$ $6432,-1.7089681206 \backslash \mathrm{H},-4.048613932,0.0653601597,-1.0272541922 \backslash \mathrm{C},-6.8788$ $766819,1.4806364486,0.4081540398 \backslash \mathrm{H},-7.5679369414,0.6962147954,0.078774$ $6248 \backslash \mathrm{H},-7.1807909808,1.7981904407,1.4113618435 \backslash \mathrm{H},-6.9967773105,2.33633$ $45588,-0.2661485557 \backslash \backslash$ Version=ES64L-G09RevD.01\State $=1-\mathrm{A} \backslash \mathrm{HF}=-235.365447$ $1 \backslash M P 2=-236.1591029 \backslash \mathrm{RMSD}=3.909 \mathrm{e}-09 \backslash \mathrm{RMSF}=3.290 \mathrm{e}-06 \backslash \mathrm{Dipole}=0.0129471,-0.0$ $269682,0.0121831 \backslash \mathrm{PG}=\mathrm{C} 01 \quad[\mathrm{X}(\mathrm{C} 6 \mathrm{H} 14)] \backslash \backslash @$

\section{Product $4-\mathrm{CH} 3$}

$\mathrm{CH} 3 \mathrm{CH} 2 \mathrm{CH} 2 \mathrm{C}(\mathrm{CH} 3) 2-\mathrm{CH} 3$

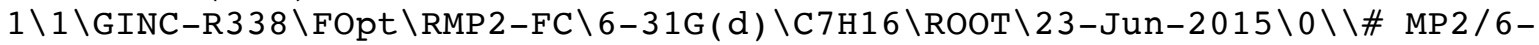
$31 \mathrm{G}(d) \quad$ SCF$=$ Tight INT(grid=ultrafine) OPT IOP(2/17=4) maxdisk=402653184 $\backslash \backslash d i M e t k . M P 2 \backslash \backslash 0,1 \backslash \mathrm{H},-2.6186793935,1.3509196013,0.1213318598 \backslash \mathrm{C},-3.01962$ $12866,1.636990616,1.1000441582 \backslash \mathrm{H},-2.9028600952,0.7649073022,1.75296916$ $38 \backslash \mathrm{C},-2.1955692291,2.7954247074,1.6596230878 \backslash \mathrm{C},-4.4979208393,2.0046062$ $582,0.9745274905 \backslash \mathrm{C},-5.4455988563,0.9246213636,0.4224251012 \backslash \mathrm{H},-4.582444$ $337,2.8940819356,0.3328201663 \backslash \mathrm{H},-4.8666506691,2.308901964,1.9652553837$ $\backslash C,-5.4474851064,-0.3116333343,1.3249683207 \backslash C,-5.0422724746,0.52012306$ $6,-0.9976491372 \backslash \mathrm{H},-2.2676114705,3.6740502337,1.0108769442 \backslash \mathrm{H},-2.5533172$ $073,3.0840968369,2.6529627383 \backslash \mathrm{H},-1.1382238643,2.5282134747,1.747582876$ $1 \backslash \mathrm{H},-5.7694083462,-0.1854442531,-1.4147379979 \backslash \mathrm{H},-5.0050003369,1.394864$ $2468,-1.6569437829 \backslash \mathrm{H},-4.0614404179,0.0360191863,-1.0181357774 \backslash \mathrm{H},-6.185$ $6413711,-1.0405946072,0.9724670168 \backslash \mathrm{H},-4.4720412614,-0.8068000042,1.336$ $3135722 \backslash \mathrm{H},-5.7054566842,-0.0421236823,2.3555217239 \backslash \mathrm{C},-6.8599951133,1.5$ $108331999,0.3856285742 \backslash \mathrm{H},-7.576353071,0.7777381661,-0.0016360126 \backslash \mathrm{H},-7$. $1865289378,1.8065559857,1.3888208236 \backslash \mathrm{H},-6.8992996309,2.396177737,-0.25$ $86462934 \backslash \backslash$ Version=ES64L-G09RevD. $01 \backslash$ State $=1-\mathrm{A} \backslash \mathrm{HF}=-274.3978104 \backslash \mathrm{MP} 2=-275$. $3283167 \backslash \mathrm{RMSD}=4.218 \mathrm{e}-09 \backslash \mathrm{RMSF}=9.547 \mathrm{e}-06 \backslash \mathrm{Dipole}=0.0187264,-0.0130808,-0.0$ $014595 \backslash P G=C 01 \quad[X(C 7 H 16)] \backslash \backslash @$

5-)

Product5-H

$\mathrm{CH} 3 \mathrm{OCH} 2-\mathrm{H}$

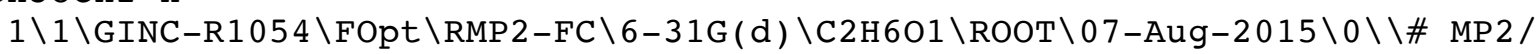
6-31G(d) SCF=Tight INT(grid=ultrafine) OPT IOP $(2 / 17=4)$ maxdisk=9395240 $96 \backslash \backslash$ diMetk.MP2 $\backslash \backslash 0,1 \backslash \mathrm{H},-2.6605723436,0.5043101132,0.6092269442 \backslash \mathrm{C},-2.919$ $7476257,1.4745732054,1.0564455586 \backslash \mathrm{H},-2.7192350131,1.4413509486,2.12791$ $3336 \backslash \mathrm{H},-2.2924267833,2.2502179582,0.5944607965 \backslash \mathrm{O},-4.2971020949,1.76372$ $66781,0.9049619859 \backslash \mathrm{C},-4.6553635775,1.8275596565,-0.4631901325 \backslash \mathrm{H},-4.099$ $4868281,2.6176877518,-0.9876776636 \backslash \mathrm{H},-4.467860997,0.8717053473,-0.9726$ $734168 \backslash \mathrm{H},-5.7218987368,2.0522583411,-0.5012154083 \backslash \backslash$ Version=ES64L-G09Re vD. $01 \backslash$ State $=1-A \backslash H F=-154.0632286 \backslash M P 2=-154.5034552 \backslash R M S D=2.506 e-09 \backslash$ RMSF $=4$ $.892 \mathrm{e}-05 \backslash \mathrm{Dipole}=0.3593128,-0.079447,-0.4293799 \backslash \mathrm{PG}=\mathrm{C} 01[\mathrm{X}(\mathrm{C} 2 \mathrm{H} 6 \mathrm{O} 1)] \backslash \backslash @$

Product5- $\mathrm{CH} 3$

$\mathrm{CH} 3 \mathrm{OCH} 2-\mathrm{CH} 3$

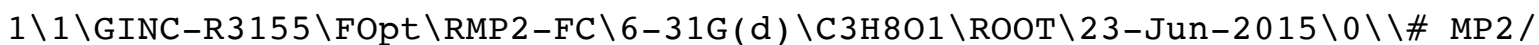
6-31G(d) SCF=Tight INT(grid=ultrafine) OPT IOP $(2 / 17=4)$ maxdisk=1342177 $28 \backslash \backslash$ diMetk.MP2 $\backslash \backslash 0,1 \backslash \mathrm{H},-2.6599309413,0.5046050487,0.606026291 \backslash \mathrm{C},-2.9185$ 
$147415,1.4744081578,1.0541135972 \backslash \mathrm{H},-2.7166023397,1.4403992397,2.125469$ $6318 \backslash \mathrm{H},-2.292069802,2.2504629076,0.5920869025 \backslash 0,-4.2970428052,1.763687$ $3444,0.9046555491 \backslash \mathrm{C},-4.6698880165,1.8305064042,-0.4643850238 \backslash \mathrm{H},-4.0836$ $179847,2.6088757433,-0.9784724881 \backslash \mathrm{H},-4.4507091223,0.8729053921,-0.9632$ $023789 \backslash \mathrm{C},-6.149789475,2.1429298324,-0.5244615816 \backslash \mathrm{H},-6.7187078608,1.361$ $9969597,-0.0155957597 \backslash \mathrm{H},-6.3520455606,3.0959927063,-0.0312815624 \backslash \mathrm{H},-6$. $4882613505,2.2051002638,-1.562633177 \backslash \backslash$ Version=ES64L-G0 9RevD. $01 \backslash$ State $=1$ $-\mathrm{A} \backslash \mathrm{HF}=-193.103225 \backslash \mathrm{MP} 2=-193.6745411 \backslash \mathrm{RMSD}=7.525 \mathrm{e}-09 \backslash \mathrm{RMSF}=6.562 \mathrm{e}-06 \backslash \mathrm{Dipol}$ $e=0.3189359,-0.0705546,-0.389649 \backslash P G=C 01[X(C 3 H 801)] \backslash \backslash @$

6-)

Product 6-H

$\mathrm{CH} 3 \mathrm{CH} 2 \mathrm{OCH} 2-\mathrm{H}$

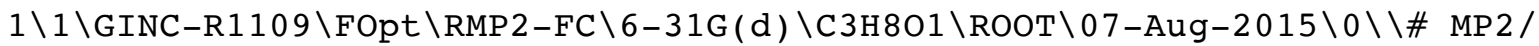
6-31G(d) SCF=Tight INT(grid=ultrafine) OPT IOP(2/17=4) maxdisk=9395240 $96 \backslash \backslash$ diMetk.MP2 $\backslash \backslash 0,1 \backslash \mathrm{H},-2.735864759,2.3509390768,-0.1757097995 \backslash \mathrm{C},-2.876$ $5198518,2.0090233994,0.8621672211 \backslash \mathrm{H},-2.2101442256,1.1450580961,1.01509$ $32647 \backslash \mathrm{C},-2.5559149931,3.1169159323,1.8425459351 \backslash 0,-4.2281132185,1.6252$ $827341,1.0702745255 \backslash \mathrm{C},-4.6144144465,0.5860865698,0.1884659093 \backslash \mathrm{H},-4.001$ $5813025,-0.3143669655,0.3354580959 \backslash \mathrm{H},-3.2198178007,3.9679687671,1.6768$ $658096 \backslash \mathrm{H},-2.6946594015,2.7633771916,2.8663955908 \backslash \mathrm{H},-1.5209267901,3.449$ $3550048,1.7222635814 \backslash \mathrm{H},-4.5302358882,0.8982440509,-0.8619908541 \backslash \mathrm{H},-5.6$ $562071559,0.3524665871,0.4118226784 \backslash \backslash$ Version=ES64L-G09RevD.01\State=1$A^{\prime} \backslash H F=-193.1032228 \backslash M P 2=-193.674541 \backslash R M S D=2.261 e-09 \backslash R M S F=4.450 e-05 \backslash D i p o l$ $\mathrm{e}=0.3086053,-0.2078584,-0.3467346 \backslash \mathrm{PG}=\mathrm{CS} \quad[\mathrm{SG}(\mathrm{C} 3 \mathrm{H} 2 \mathrm{O} 1), \mathrm{X}(\mathrm{H} 6)] \backslash \backslash @$

Product $6-\mathrm{CH} 3$

$\mathrm{CH} 3 \mathrm{CH} 2 \mathrm{OCH} 2-\mathrm{CH} 3$

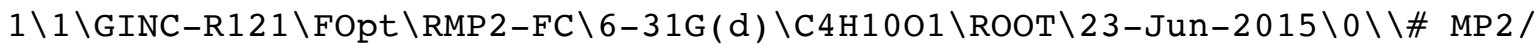
6-31G(d) SCF=Tight INT(grid=ultrafine) OPT IOP(2/17=4) maxdisk=4026531 $84 \backslash \backslash \mathrm{diMetk} . \mathrm{MP} 2 \backslash \backslash 0,1 \backslash \mathrm{H},-2.7355395393,2.3501243328,-0.1767004959 \backslash \mathrm{C},-2.87$ $58240664,2.0081583546,0.8609734413 \backslash \mathrm{H},-2.2097321805,1.144293608,1.01410$ $05752 \backslash \mathrm{C},-2.5549623935,3.1162785632,1.8414389362 \backslash 0,-4.2284328516,1.6246$ $374499,1.069868824 \backslash \mathrm{C},-4.6287395187,0.582012616,0.1906850439 \backslash \mathrm{H},-3.98667$ $61447,-0.3014291878,0.3344657166 \backslash \mathrm{H},-3.2187748722,3.9674345196,1.675789$ $0287 \backslash \mathrm{H},-2.6941029789,2.7628933417,2.8653118361 \backslash \mathrm{H},-1.5198609826,3.44888$ $07831,1.7216797494 \backslash \mathrm{H},-4.5126272624,0.904501035,-0.8561710988 \backslash \mathrm{C},-6.0746$ $430299,0.2525523326,0.4956672638 \backslash \mathrm{H},-6.1748026734,-0.0698459764,1.53421$ $46805 \backslash \mathrm{H},-6.7000259138,1.1350767978,0.34532183 \backslash \mathrm{H},-6.4333335162,-0.54820$ $49922,-0.1574255872 \backslash \backslash$ Version=ES64L-G09RevD.01\State $=1-\mathrm{A} \backslash \mathrm{HF}=-232.143107$ $4 \backslash M P 2=-232.8456436 \backslash \mathrm{RMSD}=6.141 \mathrm{e}-09 \backslash \mathrm{RMSF}=1.767 \mathrm{e}-05 \backslash \mathrm{Dipole}=0.2728119,-0.1$ $888176,-0.3117088 \backslash \mathrm{PG}=\mathrm{C} 02[\mathrm{C} 2(\mathrm{O} 1), \mathrm{X}(\mathrm{C} 4 \mathrm{H} 10)] \backslash \backslash @$

7-)

Product $7-\mathrm{H}$

$\mathrm{CH} 3 \mathrm{OC}$ ( $\mathrm{CH} 3$ ) $2-\mathrm{H}$

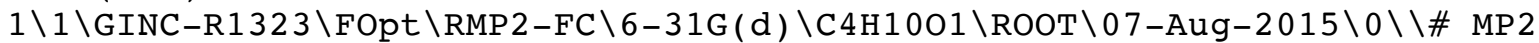
/6-31G(d) SCF=Tight INT(grid=ultrafine) OPT IOP(2/17=4) maxdisk=939524 $096 \backslash \backslash$ diMetk.MP2 $\backslash \backslash 0,1 \backslash \mathrm{H},-2.0483640542,2.2767426353,0.8269184193 \backslash \mathrm{C},-2.99$ $93052289,1.7661152485,0.6673013654 \backslash \mathrm{H},-3.4594539002,2.1566156183,-0.248$ $6483857 \backslash \mathrm{H},-2.8127150208,0.6908011992,0.5362620927 \backslash 0,-3.7850748735,2.01$ $44189871,1.8213386812 \backslash \mathrm{C},-5.0036521157,1.2666365074,1.8575181938 \backslash \mathrm{C},-5.4$ $993860168,1.3634151469,3.2884361824 \backslash \mathrm{H},-4.7795630923,0.213218882,1.6177$ $716681 \backslash \mathrm{H},-6.4213754529,0.7898243596,3.4202790445 \backslash \mathrm{H},-4.7416714785,0.981$ $2123531,3.9759617395 \backslash \mathrm{H},-5.6973003217,2.4094520679,3.5379635304 \backslash \mathrm{C},-6.02$ $39997027,1.8037856288,0.8615896762 \backslash \mathrm{H},-5.6758473591,1.7126292722,-0.170$ $2998072 \backslash \mathrm{H},-6.2139636216,2.8602313533,1.0716625174 \backslash \mathrm{H},-6.9665357611,1.25$ $41477402,0.9455600819 \backslash \backslash$ Version=ES64L-G09RevD.01 \State=1-A \HF=-232.1397 $643 \backslash \mathrm{MP} 2=-232.8449421 \backslash \mathrm{RMSD}=4.764 \mathrm{e}-09 \backslash \mathrm{RMSF}=5.870 \mathrm{e}-06 \backslash \mathrm{Dipole}=-0.1394812,-$ $0.3138397,-0.3756772 \backslash \mathrm{PG}=\mathrm{C} 01[\mathrm{X}(\mathrm{C} 4 \mathrm{H} 10 \mathrm{O} 1)] \backslash \backslash \mathrm{a}$ 
Product 7- $\mathrm{CH} 3$

$\mathrm{CH} 3 \mathrm{OC}(\mathrm{CH} 3) 2-\mathrm{CH} 3$

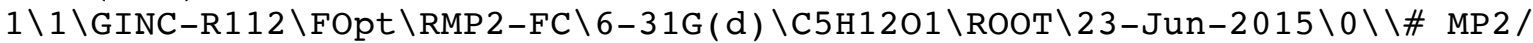
6-31G(d) SCF=Tight INT(grid=ultrafine) OPT IOP $(2 / 17=4)$ maxdisk=4026531 $84 \backslash \backslash$ diMetk.MP2 $\backslash \backslash 0,1 \backslash \mathrm{H},-2.1046211049,2.3869223385,0.8476645035 \backslash \mathrm{C},-3.011$ $1043339,1.8114303136,0.6525321229 \backslash \mathrm{H},-3.5485382455,2.2787337919,-0.1811$ $450028 \backslash \mathrm{H},-2.7230397933,0.7939163419,0.3628799996 \backslash \mathrm{O},-3.7500348229,1.844$ $3220997,1.8642723476 \backslash \mathrm{C},-5.0139605359,1.1505747427,1.8712952369 \backslash \mathrm{C},-5.51$ $85544193,1.3791743467,3.2894861728 \backslash \mathrm{C},-4.8212774415,-0.3421890792,1.612$ $4231218 \backslash \mathrm{H},-5.7610791163,-0.8775934782,1.778482935 \backslash \mathrm{H},-4.5049724045,-0.5$ $413849956,0.5847972507 \backslash \mathrm{H},-4.0659831804,-0.7419872207,2.295185141 \backslash \mathrm{H},-6$. $4900050252,0.8989518413,3.4396516703 \backslash \mathrm{H},-4.8039871999,0.9681360318,4.00$ $73938205 \backslash \mathrm{H},-5.618964966,2.4511975388,3.4782597791 \backslash \mathrm{C},-5.9806238126,1.76$ $41149025,0.8604930967 \backslash \mathrm{H},-5.6682455062,1.5755345538,-0.1703273544 \backslash \mathrm{H},-6$. $0416977337,2.8451242167,1.0157717557 \backslash \mathrm{H},-6.9796503578,1.3357017138,0.98$ $65434032 \backslash \backslash$ Version=ES64L-G09RevD. $01 \backslash$ State $=1-\mathrm{A} \backslash \mathrm{HF}=-271.173658 \backslash \mathrm{MP} 2=-272.0$ $149569 \backslash \mathrm{RMSD}=7.783 \mathrm{e}-09 \backslash \mathrm{RMSF}=4.802 \mathrm{e}-07 \backslash \mathrm{Dipole}=-0.1762066,-0.244411,-0.40$ $51053 \backslash P G=C 01 \quad[X(C 5 H 1201)] \backslash \backslash @$

8-)

Product $8-\mathrm{H}$

$\mathrm{CH} 3 \mathrm{CH} 2 \mathrm{OC}(\mathrm{CH} 3) 2-\mathrm{H}$

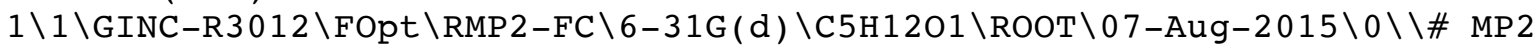
/6-31G(d) SCF=Tight INT (grid=ultrafine) OPT IOP $(2 / 17=4)$ maxdisk=939524 $096 \backslash \backslash d i M e t k . M P 2 \backslash \backslash 0,1 \backslash \mathrm{H},-2.545923424,0.8730281595,1.3134556929 \backslash \mathrm{C},-3.103$ $8437568,1.7990550731,1.5113682697 \backslash \mathrm{H},-3.3419561721,1.8180243476,2.58665$ $06334 \backslash \mathrm{C},-2.274992834,3.0058212166,1.1224152064 \backslash 0,-4.3042270818,1.84916$ $76992,0.7487570018 \backslash \mathrm{C},-5.2886731787,0.8866385287,1.1387660668 \backslash \mathrm{C},-4.9050$ $072048,-0.5205315299,0.6983797957 \backslash \mathrm{C},-6.5861331621,1.3491410427,0.50164$ $55843 \backslash \mathrm{H},-2.0543308761,2.9785325402,0.0531361081 \backslash \mathrm{H},-2.8246536902,3.9242$ $66884,1.3386347588 \backslash \mathrm{H},-1.3310729106,3.0196844095,1.6751868473 \backslash \mathrm{H},-7.4113$ $717094,0.684027558,0.7722663332 \backslash \mathrm{H},-6.8254752966,2.363440296,0.82863324$ $85 \backslash \mathrm{H},-6.4795994571,1.3523206005,-0.5866297854 \backslash \mathrm{H},-5.6973884592,-1.23116$ $62179,0.953205211 \backslash \mathrm{H},-4.7575781372,-0.5332705312,-0.3853848893 \backslash \mathrm{H},-3.983$ $8310656,-0.8610699997,1.1776489881 \backslash \mathrm{H},-5.3909015837,0.9103079232,2.2368$ $859287 \backslash \backslash$ Version=ES64L-G09RevD.01 \State $=1-\mathrm{A} \backslash \mathrm{HF}=-271.1795507 \backslash \mathrm{MP} 2=-272.01$ $61515 \backslash \mathrm{RMSD}=5.368 \mathrm{e}-09 \backslash \mathrm{RMSF}=1.080 \mathrm{e}-05 \backslash \mathrm{Dipole}=0.0726439,-0.3030268,0.3357$ $884 \backslash P G=C 01 \quad[X(\mathrm{C} 5 \mathrm{H} 12 \mathrm{O} 1)] \backslash \backslash @$

\section{Product $8-\mathrm{CH} 3$}

$\mathrm{CH} 3 \mathrm{CH} 2 \mathrm{OC}$ ( $\mathrm{CH} 3) 2-\mathrm{CH} 3$

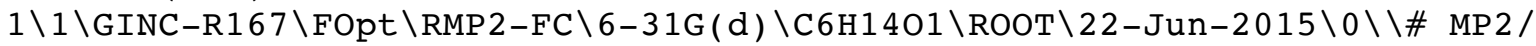
6-31G(d) SCF=Tight INT(grid=ultrafine) OPT IOP $(2 / 17=4)$ maxdisk=4026531 $84 \backslash \backslash$ diMetk.MP $2 \backslash \backslash 0,1 \backslash \mathrm{H},-2.580014933,0.8759249822,0.9383381278 \backslash \mathrm{C},-3.0631$ $951562,1.7343234522,1.4246987615 \backslash \mathrm{H},-3.0702544202,1.5434767872,2.506395$ $114 \backslash \mathrm{C},-2.2992222538,3.0076862703,1.1213625926 \backslash 0,-4.3827950814,1.925724$ $6399,0.9230475714 \backslash \mathrm{C},-5.3653864055,0.9204513094,1.2483089261 \backslash \mathrm{C},-4.89276$ $05336,-0.4723685336,0.8394879037 \backslash \mathrm{C},-6.5697635475,1.3362912519,0.414709$ $7662 \backslash \mathrm{H},-2.3041454935,3.1978128055,0.045869403 \backslash \mathrm{H},-2.7686845617,3.855936$ $2821,1.6240205651 \backslash \mathrm{H},-1.2619761457,2.9254759726,1.4596773851 \backslash \mathrm{H},-7.41472$ $52752,0.6628842698,0.5871937911 \backslash \mathrm{H},-6.8677976975,2.3542902445,0.6791587$ $567 \backslash \mathrm{H},-6.3108115743,1.3177215167,-0.6471780456 \backslash \mathrm{H},-5.7100970982,-1.1922$ $192593,0.9463508065 \backslash \mathrm{H},-4.5702667593,-0.4648546108,-0.2059376349 \backslash \mathrm{H},-4.0$ $616356068,-0.8186365075,1.460177631 \backslash C,-5.7098515843,0.9719014049,2.735$ $6992385 \backslash \mathrm{H},-4.8662219498,0.6643294166,3.35967035 \backslash \mathrm{H},-5.9951033995,1.9907$ $846884,3.0121137043 \backslash \mathrm{H},-6.5465405229,0.3012336168,2.9552652858 \backslash \backslash$ Version $=\mathrm{ES} 64 \mathrm{~L}-\mathrm{G} 09$ RevD.01 $\backslash$ State $=1-\mathrm{A} \backslash \mathrm{HF}=-310.213267 \backslash \mathrm{MP} 2=-311.1862164 \backslash \mathrm{RMSD}=4.833$ e-09\RMSF $=1.437 \mathrm{e}-06 \backslash \mathrm{Dipole}=0.0941522,-0.3744964,0.2527635 \backslash P G=C 01 \quad[\mathrm{X}(\mathrm{C} 6$ H1401) ] \\@ 
9-)

Product $9-\mathrm{H}$

HOCH $2 \mathrm{CH} 2-\mathrm{H}$

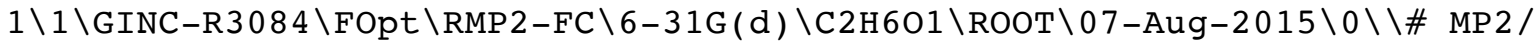
6-31G(d) SCF=Tight INT(grid=ultrafine) OPT IOP(2/17=4) maxdisk=9395240 $96 \backslash \backslash$ diMetk.MP $2 \backslash \backslash 0,1 \backslash \mathrm{O},-2.9438675308,2.81398893,0.8605415827 \backslash \mathrm{H},-2.14850$ $35765,2.4653247995,1.2967320172 \backslash C,-4.0333819698,1.9733857654,1.2440373$ $836 \backslash \mathrm{C},-3.8511370211,0.5411990067,0.7690949728 \backslash \mathrm{H},-4.9140961064,2.421878$ $7034,0.7789385727 \backslash \mathrm{H},-4.1856740776,2.0012380623,2.3324901588 \backslash \mathrm{H},-3.71340$ $51581,0.5221176087,-0.3141940738 \backslash \mathrm{H},-2.9710021058,0.0866322477,1.235868$ $8898 \backslash \mathrm{H},-4.7207154539,-0.0718181238,1.0256454963 \backslash \backslash$ Version=ES $64 \mathrm{~L}-\mathrm{G} 09 \mathrm{RevD}$ $.01 \backslash$ State $=1-\mathrm{A} \backslash \mathrm{HF}=-154.0741406 \backslash \mathrm{MP} 2=-154.5171225 \backslash \mathrm{RMSD}=5.063 \mathrm{e}-09 \backslash \mathrm{RMSF}=1.5$ $51 \mathrm{e}-05 \backslash \mathrm{Dipole}=0.0517377,-0.5810967,0.3984413 \backslash \mathrm{PG}=\mathrm{C} 01 \quad[\mathrm{X}(\mathrm{C} 2 \mathrm{H} 6 \mathrm{O} 1)] \backslash \backslash @$

\section{Product9-CH3}

HOCH $2 \mathrm{CH} 2-\mathrm{CH} 3$

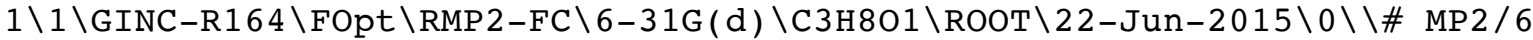
$-31 \mathrm{G}(\mathrm{d}) \quad \mathrm{SCF}=\mathrm{Tight}$ INT (grid=ultrafine) OPT IOP(2/17=4) maxdisk=40265318 $4 \backslash \backslash$ diMetk.MP2 $\backslash \backslash 0,1 \backslash 0,-2.9134707516,2.7452621515,0.8531123702 \backslash \mathrm{H},-2.1419$ $147743,2.344876869,1.2866568303 \backslash C,-4.0657304235,1.9947357932,1.2394215$ $229 \backslash \mathrm{C},-5.266140338,2.6219017944,0.5591590504 \backslash \mathrm{H},-3.9720988431,0.9409614$ $261,0.9355354095 \backslash \mathrm{H},-4.2026730564,2.0134880557,2.3314300931 \backslash \mathrm{H},-5.320151$ $0035,3.6728322756,0.8612399416 \backslash \mathrm{H},-5.0917155106,2.6102009622,-0.5216167$ $044 \backslash \mathrm{C},-6.5665326598,1.8995918811,0.899500627 \backslash \mathrm{H},-6.5293432502,0.8515630$ $088,0.5862537489 \backslash \mathrm{H},-6.7596666938,1.9212844362,1.9765661295 \backslash \mathrm{H},-7.418267$ $6952,2.3670633461,0.3987059811 \backslash \backslash$ Version=ES64L-G09RevD. $01 \backslash$ State $=1-\mathrm{A} \backslash \mathrm{HF}=$ $-193.1089029 \backslash M P 2=-193.6822945 \backslash R M S D=5.050 e-09 \backslash R M S F=2.460 e-05 \backslash D i p o l e=0.0$ $099768,-0.5009427,0.3854835 \backslash P G=C 01[X(C 3 H 801)] \backslash \backslash @$

$10-)$

Product $10-\mathrm{H}$

HOCH $2 \mathrm{C}(\mathrm{CH} 3) 2-\mathrm{H}$

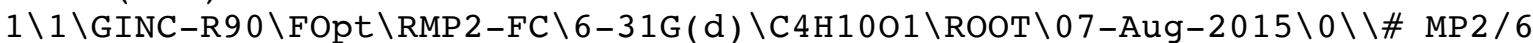
$-31 \mathrm{G}(\mathrm{d})$ SCF=Tight INT (grid=ultrafine) OPT IOP(2/17=4) maxdisk=93952409 $6 \backslash \backslash$ diMetk.MP $2 \backslash \backslash 0,1 \backslash 0,-2.8265568748,2.0620909655,0.7826532098 \backslash \mathrm{H},-2.5466$ $463431,1.3562921195,1.3900623672 \backslash \mathrm{C},-4.2497815463,2.1476485851,0.875252$ $053 \backslash \mathrm{C},-4.962175824,0.8870604043,0.3937955242 \backslash \mathrm{H},-4.5289220758,2.9948827$ $816,0.2402328328 \backslash \mathrm{H},-4.5609761044,2.3871474998,1.9037722091 \backslash \mathrm{H},-4.628757$ $2478,0.0564402499,1.0352997286 \backslash \mathrm{C},-4.5843002899,0.5662408718,-1.0487232$ $235 \backslash \mathrm{H},-5.0346647541,-0.3770228603,-1.3730268948 \backslash \mathrm{H},-4.9406725978,1.3582$ $617982,-1.7169635225 \backslash \mathrm{H},-3.500398543,0.4947714974,-1.159338495 \backslash \mathrm{C},-6.472$ $7099644,1.0404819339,0.5592485154 \backslash \mathrm{H},-6.9945001583,0.1316907242,0.24545$ $3662 \backslash \mathrm{H},-6.7454208352,1.2447652003,1.5998013752 \backslash \mathrm{H},-6.8424848412,1.86775$ $12287,-0.0564793415 \backslash \backslash$ Version=ES64L-G09RevD. $01 \backslash$ State $=1-A \backslash H F=-232.144081$ $3 \backslash M P 2=-232.8518554 \backslash \mathrm{RMSD}=8.913 e-09 \backslash \mathrm{RMSF}=7.608 \mathrm{e}-06 \backslash \mathrm{Dipole}=-0.3737618,-0$. $4376447,0.3908548 \backslash \mathrm{PG}=\mathrm{C} 01 \quad[\mathrm{X}(\mathrm{C} 4 \mathrm{H} 10 \mathrm{O} 1)] \backslash \backslash @$

\section{Product $10-\mathrm{CH} 3$}

$\mathrm{HOCH} 2 \mathrm{C}(\mathrm{CH} 3) 2-\mathrm{CH} 3$

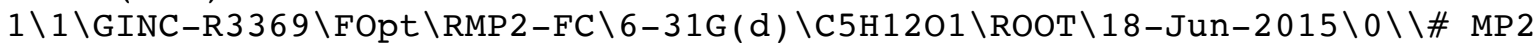
/6-31G(d) SCF=Tight INT(grid=ultrafine) OPT IOP $(2 / 17=4)$ maxdisk=134217 $728 \backslash \backslash$ diMetk.MP $2 \backslash \backslash 0,1 \backslash 0,-2.8241595097,2.0651708826,0.7653402571 \backslash \mathrm{H},-2.50$ $95685686,1.4824162693,1.4757990932 \backslash \mathrm{C},-4.2482412473,2.1212463179,0.8653$ $234943 \backslash \mathrm{C},-4.9530305212,0.8402362252,0.4076582868 \backslash \mathrm{H},-4.5501274909,2.954$ $9822312,0.2227964969 \backslash \mathrm{H},-4.5580703595,2.3647868951,1.894165759 \backslash \mathrm{C},-4.511$ $5905216,-0.3353008844,1.2811641583 \backslash \mathrm{C},-4.6006913464,0.5606674751,-1.052$ $7219163 \backslash \mathrm{H},-5.063989093,-0.372098075,-1.3919190616 \backslash \mathrm{H},-4.9566849914,1.37$ $05919338,-1.6989872314 \backslash \mathrm{H},-3.5178786923,0.480210621,-1.1760925154 \backslash \mathrm{H},-5$. $0461456645,-1.2476857598,0.9979675583 \backslash \mathrm{H},-3.441311189,-0.5353154191,1.1$ 
$647963962 \backslash \mathrm{H},-4.7182934726,-0.1409463624,2.3405006517 \backslash \mathrm{C},-6.4604282449,1$ $.0503418059,0.5544833275 \backslash \mathrm{H},-7.0070564224,0.1627127596,0.219457336 \backslash \mathrm{H},-6$ $.7351840163,1.2416188777,1.5979892661 \backslash \mathrm{H},-6.7982786485,1.9007442064,-0$. $0480713568 \backslash \backslash$ Version=ES64L-G09RevD. 01 \State $=1-\mathrm{A} \backslash \mathrm{HF}=-271.1781841 \backslash \mathrm{MP} 2=-27$ $2.022372 \backslash \mathrm{RMSD}=2.627 \mathrm{e}-09 \backslash \mathrm{RMSF}=8.772 \mathrm{e}-06 \backslash \mathrm{Dipole}=-0.3441736,-0.3717013,0$. $4358127 \backslash P G=C 01 \quad[X(C 5 H 1201)] \backslash \backslash @$

11

Product $11-\mathrm{H}$

$\mathrm{CH} 3 \mathrm{OCH} 2 \mathrm{CH} 2-\mathrm{H}$

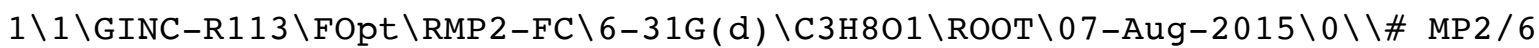
$-31 \mathrm{G}(\mathrm{d}) \mathrm{SCF}=\mathrm{Tight}$ INT(grid=ultrafine) OPT IOP(2/17=4) maxdisk=93952409

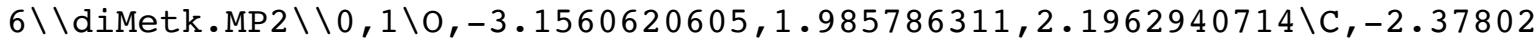
$36389,3.0664170718,1.7133766716 \backslash \mathrm{C},-4.3994457357,1.8939948212,1.5156582$ $267 \backslash \mathrm{C},-5.1562348992,0.7177252446,2.0943942898 \backslash \mathrm{H},-4.9697589549,2.828098$ $3398,1.6424676703 \backslash \mathrm{H},-4.2302234353,1.7582111434,0.4354564511 \backslash \mathrm{H},-5.32147$ $14111,0.867347632,3.1633948539 \backslash \mathrm{H},-1.4483222308,3.0667307571,2.28378210$ $67 \backslash \mathrm{H},-2.1473005735,2.9503305109,0.6449893542 \backslash \mathrm{H},-2.8912696347,4.0277160$ $838,1.8570861412 \backslash \mathrm{H},-4.58199453,-0.2010162014,1.9579405767 \backslash \mathrm{H},-6.1256348$ $954,0.6034772858,1.6010055863 \backslash \backslash$ Version=ES64L-G09RevD . 01 \State=1-A $\backslash H F=-$ $193.1032258 \backslash M P 2=-193.6745411 \backslash \mathrm{RMSD}=2.349 e-09 \backslash \mathrm{RMSF}=8.832 e-06 \backslash \mathrm{Dipole}=-0.1$ $49787,0.3139588,-0.3707929 \backslash \mathrm{PG}=\mathrm{C} 01 \quad[\mathrm{X}(\mathrm{C} 3 \mathrm{H} 8 \mathrm{O} 1)] \backslash \backslash \mathrm{Q}$

\section{Product $11-\mathrm{CH} 3$}

$\mathrm{CH} 3 \mathrm{OCH} 2 \mathrm{CH} 2-\mathrm{CH} 3$

$1 \backslash 1 \backslash G I N C-R 3517 \backslash F O p t \backslash R M P 2-F C \backslash 6-31 G(d) \backslash C 4 H 1001 \backslash R O O T \backslash 18-J u n-2015 \backslash 0 \backslash \backslash \#$ MP 2 /6-31G(d) SCF=Tight INT(grid=ultrafine) OPT IOP(2/17=4) maxdisk=134217 $728 \backslash \backslash$ diMetk.MP2 $\backslash \backslash 0,1 \backslash 0,-3.0466627071,1.9131217719,2.0285687956 \backslash \mathrm{C},-2.38$ $6697601,3.1363739413,1.7542571711 \backslash \mathrm{C},-4.3707544818,1.9095627864,1.51650$ $13123 \backslash \mathrm{C},-5.0040250286,0.5732608187,1.8501349334 \backslash \mathrm{H},-4.9538406975,2.7342$ $519275,1.9599936769 \backslash \mathrm{H},-4.3583982125,2.0687699964,0.425037652 \backslash \mathrm{H},-4.9757$ $077448,0.4404956452,2.9364302283 \backslash \mathrm{H},-1.3841738856,3.0566516584,2.176672$ $4783 \backslash \mathrm{H},-2.3107445724,3.3187186673,0.673037617 \backslash \mathrm{H},-2.9077702023,3.986826$ $6172,2.216069096 \backslash \mathrm{H},-4.3851319757,-0.2186217308,1.4161551452 \backslash \mathrm{C},-6.43789$ $1536,0.4723526707,1.3369692504 \backslash \mathrm{H},-7.0700598008,1.2492230775,1.77799874$ $77 \backslash \mathrm{H},-6.4763454561,0.5857582005,0.2491624334 \backslash \mathrm{H},-6.8770090979,-0.496915$ $0482,1.5871014624 \backslash \backslash$ Version=ES64L-G09RevD.01\State $=1-\mathrm{A} \backslash \mathrm{HF}=-232.1377768 \backslash$ $M P 2=-232.8399056 \backslash R M S D=7.483 e-09 \backslash R M S F=1.616 e-05 \backslash D i p o l e=-0.2149341,0.360$ $8611,-0.2398715 \backslash \mathrm{PG}=\mathrm{C} 01 \quad[\mathrm{X}(\mathrm{C} 4 \mathrm{H} 1001)] \backslash \backslash @$

$12-$ )

Product $12-\mathrm{H}$

$\mathrm{CH} 3 \mathrm{OCH} 2 \mathrm{C}$ ( $\mathrm{CH} 3) 2-\mathrm{H}$

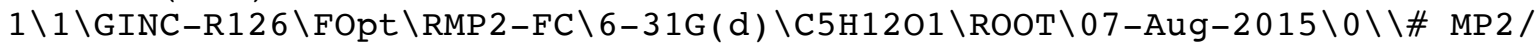
6-31G(d) SCF=Tight INT(grid=ultrafine) OPT IOP(2/17=4) maxdisk=9395240

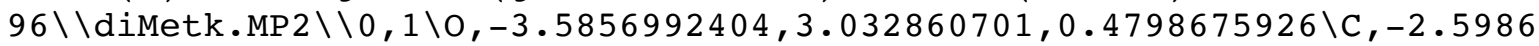
$895059,2.4644806427,1.322803327 \backslash \mathrm{C},-4.8600987999,2.4472290456,0.7009179$ $478 \backslash \mathrm{C},-5.8724455875,3.1197827323,-0.2110104317 \backslash \mathrm{H},-4.819008396,1.363482$ $1232,0.4987324049 \backslash \mathrm{H},-5.161241918,2.5750610245,1.755874688 \backslash \mathrm{H},-5.5169226$ $837,2.9786084634,-1.2398463489 \backslash \mathrm{C},-5.9534322213,4.6161138868,0.07600027$ $1 \backslash \mathrm{H},-6.6537618331,5.1066373692,-0.6074002563 \backslash \mathrm{H},-6.3057263281,4.7876208$ $977,1.0998122676 \backslash \mathrm{H},-4.9733748054,5.0844954007,-0.0315101676 \backslash \mathrm{H},-1.65757$ $66004,2.9642713105,1.0895167947 \backslash \mathrm{H},-2.8390509575,2.6201267846,2.3838464$ $662 \backslash \mathrm{H},-2.4902531936,1.3855669638,1.1436062375 \backslash \mathrm{C},-7.2359461168,2.447148$ $1819,-0.0645600375 \backslash \mathrm{H},-7.6120774788,2.5558599103,0.9587637842 \backslash \mathrm{H},-7.9671$ $64965,2.9030842926,-0.7384838453 \backslash \mathrm{H},-7.1845093682,1.3782312692,-0.29577$ $26942 \backslash \backslash$ Version=ES64L-G09RevD.01\State $=1-\mathrm{A} \backslash \mathrm{HF}=-271.1728977 \backslash \mathrm{MP} 2=-272.009$ $1998 \backslash \mathrm{RMSD}=9.067 \mathrm{e}-09 \backslash \mathrm{RMSF}=6.532 \mathrm{e}-06 \backslash \mathrm{Dipole}=-0.0381515,-0.3568933,0.3030$ $155 \backslash P G=C 01 \quad[X(C 5 H 1201)] \backslash \backslash @$ 
Product $12-\mathrm{CH} 3$

$\mathrm{CH} 3 \mathrm{OCH} 2 \mathrm{C}(\mathrm{CH} 3) 2-\mathrm{CH} 3$

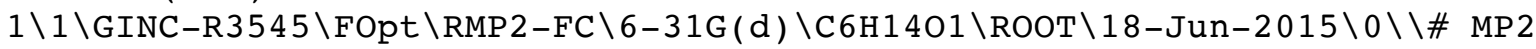
$16-31 \mathrm{G}(\mathrm{d}) \quad \mathrm{SCF}=\mathrm{Tight}$ INT(grid=ultrafine) OPT IOP $(2 / 17=4)$ maxdisk $=402653$ $184 \backslash \backslash$ diMetk.MP $2 \backslash \backslash 0,1 \backslash 0,-3.57949002,3.0484518076,0.4939608268 \backslash \mathrm{C},-2.6085$ $339748,2.4622860318,1.3439093915 \backslash \mathrm{C},-4.8560361558,2.4532219366,0.674116$ $1964 \backslash \mathrm{C},-5.8616091891,3.1175642013,-0.2598506117 \backslash \mathrm{H},-4.8020375592,1.3711$ $554408,0.4608953077 \backslash \mathrm{H},-5.1825141266,2.5689767415,1.7225711674 \backslash \mathrm{C},-5.416$ $91365,2.9245239578,-1.7100758654 \backslash C,-5.9523395913,4.6090839529,0.064651$ $4858 \backslash \mathrm{H},-6.6756737837,5.1007839262,-0.594730906 \backslash \mathrm{H},-6.2787147508,4.76247$ $64923,1.099578753 \backslash \mathrm{H},-4.9799644782,5.0904163628,-0.0622515544 \backslash \mathrm{H},-6.1325$ $126249,3.3918554562,-2.3951197467 \backslash \mathrm{H},-4.4334489801,3.3709638897,-1.8737$ $538362 \backslash \mathrm{H},-5.3558612726,1.8590213765,-1.959276382 \backslash \mathrm{H},-1.6649598072,2.972$ $1505145,1.1446980939 \backslash \mathrm{H},-2.8735950349,2.5894112713,2.402822494 \backslash \mathrm{H},-2.491$ $7891611,1.3889650426,1.1387958178 \backslash \mathrm{C},-7.2222584867,2.4540769465,-0.0405$ $85847 \backslash \mathrm{H},-7.5601049822,2.5761095182,0.9945880085 \backslash \mathrm{H},-7.9750819907,2.9047$ $831382,-0.6955176169 \backslash \mathrm{H},-7.1805703799,1.3820519946,-0.2633851763 \backslash \backslash$ Versi on $=E S 64 \mathrm{~L}-\mathrm{G} 0$ 9RevD. $01 \backslash$ State $=1-\mathrm{A} \backslash \mathrm{HF}=-310.2072124 \backslash \mathrm{MP} 2=-311.1801378 \backslash \mathrm{RMSD}=8$. $357 \mathrm{e}-09 \backslash \mathrm{RMSF}=1.929 \mathrm{e}-05 \backslash \mathrm{Dipole}=0.0002912,-0.3447879,0.3276875 \backslash \mathrm{PG}=\mathrm{C} 01[\mathrm{X}$ ( $6 \mathrm{H} 1401)] \backslash \backslash @$

13-)

Product $13-\mathrm{H}$

HOOCH $2-\mathrm{H}$

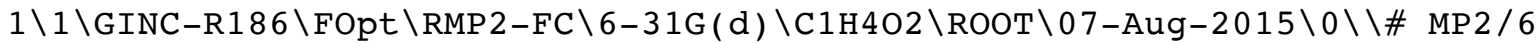
$-31 \mathrm{G}(\mathrm{d}) \quad \mathrm{SCF}=\mathrm{Tight}$ INT(grid=ultrafine) OPT IOP(2/17=4) maxdisk=93952409 $6 \backslash \backslash$ diMetk.MP2 $\backslash \backslash 0,1 \backslash \mathrm{O},-3.2944128261,2.1334335291,2.0369985493 \backslash \mathrm{H},-2.9695$ $011217,1.3965156598,2.5897704865 \backslash 0,-3.9288493028,1.3406068664,0.973358$ $5117 \backslash \mathrm{C},-5.2751021214,1.7975134188,0.9443729631 \backslash \mathrm{H},-5.3180958315,2.87074$ $14769,0.7399785113 \backslash \mathrm{H},-5.7901660735,1.5759338173,1.8841937779 \backslash \mathrm{H},-5.7295$ $28723,1.2403452318,0.1220002002 \backslash \backslash$ Version=ES64L-G09RevD.01 \State $=1-\mathrm{A} \backslash \mathrm{HF}$ $=-189.7916821 \backslash M P 2=-190.2900714 \backslash \mathrm{RMSD}=9.468 \mathrm{e}-09 \backslash \mathrm{RMSF}=1.177 \mathrm{e}-05 \backslash \mathrm{Dipole}=-0$ $.4469125,-0.3050921,0.3614929 \backslash \mathrm{PG}=\mathrm{C} 01 \quad[\mathrm{X}(\mathrm{C} 1 \mathrm{H} 4 \mathrm{O} 2)] \backslash \backslash @$

Product $13-\mathrm{CH} 3$

HOOCH2-CH 3

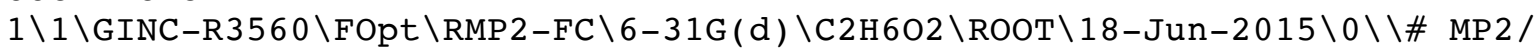
6-31G(d) SCF=Tight INT(grid=ultrafine) OPT IOP $(2 / 17=4)$ maxdisk=1342177 $28 \backslash \backslash$ diMetk.MP2 $\backslash \backslash 0,1 \backslash 0,-3.2770395358,2.1387439128,2.0179442757 \backslash \mathrm{H},-3.010$ $7693325,1.407657497,2.6087220628 \backslash 0,-3.9220431653,1.3419026474,0.963272$ $6725 \backslash \mathrm{C},-5.2785512527,1.78733407,0.9336793015 \backslash \mathrm{H},-5.3035445433,2.8694440$ $875,0.7655752654 \backslash \mathrm{H},-5.7623410937,1.5730135653,1.8945839771 \backslash \mathrm{C},-5.930261$ $081,1.0287679136,-0.2059247404 \backslash \mathrm{H},-5.881335172,-0.047927476,-0.02944327$ $32 \backslash \mathrm{H},-6.9805592629,1.3197223836,-0.2921829173 \backslash \mathrm{H},-5.4271955609,1.252461$ $3989,-1.1487266242 \backslash \backslash$ Version $=E S 64 \mathrm{~L}-\mathrm{G} 09$ RevD. $01 \backslash$ State $=1-\mathrm{A} \backslash \mathrm{HF}=-228.8314483$ $\backslash M P 2=-229.4609188 \backslash \mathrm{RMSD}=6.318 \mathrm{e}-09 \backslash \mathrm{RMSF}=4.943 \mathrm{e}-05 \backslash \mathrm{Dipole}=-0.5015054,-0.3$ $361379,0.3300632 \backslash \mathrm{PG}=\mathrm{C} 01 \quad[\mathrm{X}(\mathrm{C} 2 \mathrm{H} 6 \mathrm{O} 2)] \backslash \backslash @$

14-)

Product $14-\mathrm{H}$

$\mathrm{HOOC}(\mathrm{CH} 3) 2-\mathrm{H}$

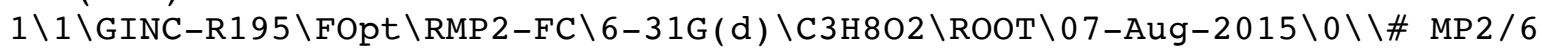
$-31 \mathrm{G}(\mathrm{d}) \mathrm{SCF}=\mathrm{Tight}$ INT (grid=ultrafine) OPT IOP(2/17=4) maxdisk=93952409 $6 \backslash \backslash$ diMetk.MP $2 \backslash \backslash 0,1 \backslash 0,-3.3421296245,2.3736720561,1.8685420773 \backslash \mathrm{H},-2.7783$ $724867,1.6058944424,2.0859675283 \backslash 0,-3.9482434552,1.8447992247,0.635250$ $8254 \backslash \mathrm{C},-5.3581767711,1.7384929522,0.8917033761 \backslash \mathrm{C},-5.8943487374,1.03985$ $18163,-0.3462499342 \backslash \mathrm{C},-5.9768841448,3.1052020375,1.1205928312 \backslash \mathrm{H},-7.052$ $050367,3.0118673906,1.3029306197 \backslash \mathrm{H},-5.5168466403,3.5923916453,1.981692$ $7769 \backslash \mathrm{H},-5.8215482869,3.7311378724,0.2375248416 \backslash \mathrm{H},-6.9724838183,0.88628$ $20424,-0.2488350687 \backslash \mathrm{H},-5.7085743398,1.653592267,-1.2315674585 \backslash \mathrm{H},-5.412$ 
$1487514,0.0693199825,-0.4844034524 \backslash \mathrm{H},-5.5000235765,1.1049402707,1.7781$

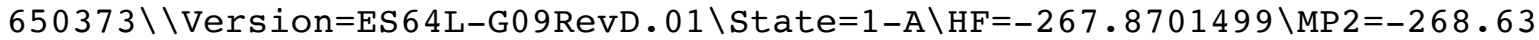
$36575 \backslash \mathrm{RMSD}=4.221 \mathrm{e}-09 \backslash \mathrm{RMSF}=1.282 \mathrm{e}-05 \backslash \mathrm{Dipole}=-0.2865781,-0.5675085,0.238$ $3888 \backslash \mathrm{PG}=\mathrm{C} 01 \quad[\mathrm{X}(\mathrm{C} 3 \mathrm{H} 8 \mathrm{O} 2)] \backslash \backslash @$

\section{Product $14-\mathrm{CH} 3$}

$\mathrm{HOOC}$ ( $\mathrm{CH} 3$ ) $2-\mathrm{CH} 3$

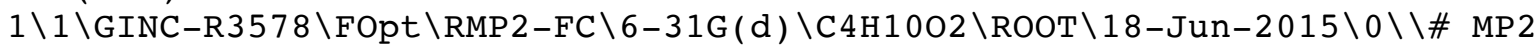
/6-31G(d) SCF=Tight INT(grid=ultrafine) OPT IOP(2/17=4) maxdisk=268435 $456 \backslash \backslash$ diMetk.MP2 \\0,1\O,-3.3180178161,2.4410662186, $1.7986094966 \backslash \mathrm{H},-2.75$ $20861529,1.6960160666,2.0797994989 \backslash 0,-3.9673554728,1.7897786567,0.6468$ $659621 \backslash \mathrm{C},-5.3829395031,1.6939837061,0.9322371296 \backslash \mathrm{C},-5.902901615,1.0473$ $107124,-0.345521681 \backslash \mathrm{C},-5.9673109264,3.085241913,1.1291348104 \backslash \mathrm{H},-7.0521$ $353957,3.026839165,1.2621736418 \backslash \mathrm{H},-5.5309618555,3.5598003495,2.0100892$ $205 \backslash \mathrm{H},-5.7514858441,3.7051864443,0.2549405995 \backslash \mathrm{H},-6.9870778898,0.918822$ $2173,-0.2807919295 \backslash \mathrm{H},-5.6744186728,1.6776084607,-1.208713277 \backslash \mathrm{H},-5.4417$ $253065,0.0671552956,-0.4940825869 \backslash \mathrm{C},-5.6188911068,0.8007637247,2.14239$ $82817 \backslash \mathrm{H},-5.1998710085,1.2575607037,3.0413952765 \backslash \mathrm{H},-6.6906312895,0.6477$ $32128,2.3006075314 \backslash \mathrm{H},-5.1489301444,-0.1749357623,1.9850280255 \backslash \backslash$ Version $=\mathrm{ES} 64 \mathrm{~L}-\mathrm{G} 09$ RevD $.01 \backslash$ State $=1-\mathrm{A} \backslash \mathrm{HF}=-306.906685 \backslash \mathrm{MP} 2=-307.8065451 \backslash \mathrm{RMSD}=6.738$ e-09\RMSF $=1.500 e-05 \backslash \mathrm{Dipole}=-0.2819293,-0.5321247,0.3187755 \backslash \mathrm{PG}=\mathrm{C} 01] \mathrm{X}(\mathrm{C}$ $4 \mathrm{H} 10 \mathrm{O} 2$ ) ] \\@

15-)

Product $15-\mathrm{H}$

$\mathrm{CH} 30 \mathrm{OCH} 2-\mathrm{H}$

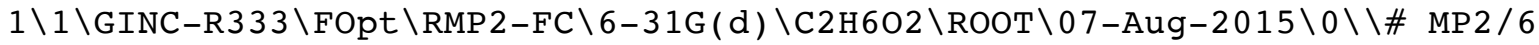
$-31 \mathrm{G}(\mathrm{d})$ SCF=Tight INT(grid=ultrafine) OPT IOP(2/17=4) maxdisk=93952409 $6 \backslash \backslash$ diMetk. MP2 $\backslash \backslash 0,1 \backslash 0,-2.9518495947,1.8378593756,1.1621228153 \backslash \mathrm{C},-2.5033$ $398021,3.0878525389,1.6715932769 \backslash 0,-4.2457922422,1.6185744836,1.830665$ $3079 \backslash \mathrm{C},-5.1635770608,1.4287984405,0.7606839185 \backslash \mathrm{H},-5.2366035913,2.32491$ $60345,0.1361441866 \backslash \mathrm{H},-1.5289479119,3.2376375907,1.2006297557 \backslash \mathrm{H},-3.1854$ $817887,3.8978615147,1.3941455117 \backslash \mathrm{H},-2.3924534881,3.0495382028,2.758840$ $9316 \backslash \mathrm{H},-4.8771472953,0.569470059,0.1479980709 \backslash \mathrm{H},-6.1184600673,1.234937$

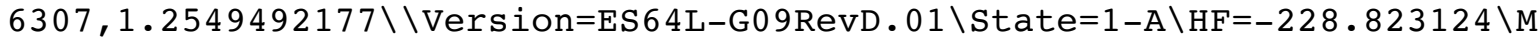
$\mathrm{P} 2=-229.4508818 \backslash \mathrm{RMSD}=8.203 e-09 \backslash \mathrm{RMSF}=7.253 e-06 \backslash \mathrm{Dipole}=-0.2203463,0.4978$ $21,-0.2631858 \backslash \mathrm{PG}=\mathrm{C} 02 \quad[\mathrm{X}(\mathrm{C} 2 \mathrm{H} 6 \mathrm{O} 2)] \backslash \backslash @$

Product $15-\mathrm{CH} 3$

$\mathrm{CH} 30 \mathrm{OCH} 2-\mathrm{CH} 3$

$1 \backslash 1 \backslash G I N C-R 2788 \backslash F O p t \backslash R M P 2-F C \backslash 6-31 G(d) \backslash C 3$ H $802 \backslash R O O T \backslash 18-J u n-2015 \backslash 0 \backslash \backslash \#$ MP $2 /$ 6-31G(d) SCF=Tight INT(grid=ultrafine) OPT IOP(2/17=4) maxdisk=2684354 $56 \backslash \backslash$ dimetk.MP2 $\backslash \backslash 0,1 \backslash 0,-2.9334390243,1.9448982402,1.1272230194 \backslash \mathrm{C},-2.355$ $7277612,3.0621701145,1.7896074119 \backslash 0,-4.2436456502,1.7778897715,1.79050$ $98625 \backslash \mathrm{C},-5.1458754347,1.4669336713,0.726573926 \backslash \mathrm{H},-4.7588007277,0.60741$ $92103,0.1675141307 \backslash \mathrm{H},-1.3629229366,3.1559472383,1.3431913261 \backslash \mathrm{H},-2.9391$ $626882,3.9714412409,1.6166627735 \backslash \mathrm{H},-2.2664231031,2.874826046,2.8636026$ $433 \backslash \mathrm{H},-6.0479435209,1.1544716742,1.2632386769 \backslash \mathrm{C},-5.4162849042,2.651451$ $3067,-0.1811116161 \backslash \mathrm{H},-5.8063590583,3.4904341527,0.4002626414 \backslash \mathrm{H},-4.4956$ $895415,2.9618875512,-0.6776925205 \backslash \mathrm{H},-6.1516766492,2.3857207822,-0.9467$ $682751 \backslash \backslash$ Version=ES64L-G09RevD.01 \State $=1-A \backslash H F=-267.862273 \backslash \mathrm{MP} 2=-268.622$ $1784 \backslash \mathrm{RMSD}=4.543 e-09 \backslash \mathrm{RMSF}=4.370 e-06 \backslash \mathrm{Dipole}=-0.1260166,0.4062671,-0.2069$ $407 \backslash \mathrm{PG}=\mathrm{C} 01 \quad[\mathrm{X}(\mathrm{C} 3 \mathrm{H} 8 \mathrm{O} 2)] \backslash \backslash \mathrm{Q}$

16-)

Product $16-\mathrm{H}$

$\mathrm{CH} 300 \mathrm{C}(\mathrm{CH} 3) 2-\mathrm{H}$

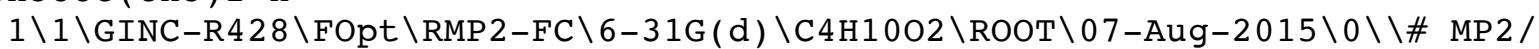
6-31G(d) SCF=Tight INT(grid=ultrafine) OPT IOP(2/17=4) maxdisk=9395240 $96 \backslash \backslash$ dimetk.MP2 $\backslash \backslash 0,1 \backslash 0,-3.5475372281,3.5060945951,1.4897622748 \backslash \mathrm{C},-2.416$ 
$8697052,2.898769329,2.1045138405 \backslash 0,-3.9640685391,2.5347995056,0.462362$ $1312 \backslash \mathrm{C},-5.2616222018,2.0637732253,0.8632369447 \backslash \mathrm{C},-5.5489749762,0.92928$ $73812,-0.1057990464 \backslash \mathrm{C},-6.2897682741,3.1785048551,0.8001322296 \backslash \mathrm{H},-2.089$ $1266615,3.6284424877,2.8492202819 \backslash \mathrm{H},-2.6872624085,1.957728176,2.594894$ $8113 \backslash \mathrm{H},-1.6223121658,2.7237711175,1.3740143066 \backslash \mathrm{H},-7.2755508863,2.80720$ $59873,1.0977933571 \backslash \mathrm{H},-6.0062153872,3.9944428593,1.4667306563 \backslash \mathrm{H},-6.3513$ $115521,3.5639003888,-0.2213902488 \backslash \mathrm{H},-6.5209098639,0.4823267627,0.12079$ $61529 \backslash \mathrm{H},-5.5714845244,1.3103623548,-1.1302156422 \backslash \mathrm{H},-4.7805833653,0.155$ $9317962,-0.0357964523 \backslash \mathrm{H},-5.1855852604,1.6752431784,1.8886734027 \backslash \backslash V e r s i$ on=ES64L-G09RevD. $01 \backslash$ State $=1-A \backslash H F=-306.901272 \backslash M P 2=-307.7948094 \backslash \mathrm{RMSD}=6.1$ $23 e-09 \backslash \mathrm{RMSF}=2.732 \mathrm{e}-06 \backslash \mathrm{Dipole}=-0.0664313,-0.4785762,0.4389679 \backslash \mathrm{PG}=\mathrm{C} 01] \mathrm{X}$ ( C4H1002 ) ] \\@

\section{Product $16-\mathrm{CH} 3$}

\section{$\mathrm{CH} 300 \mathrm{C}$ ( $\mathrm{CH} 3$ ) $2-\mathrm{CH} 3$}

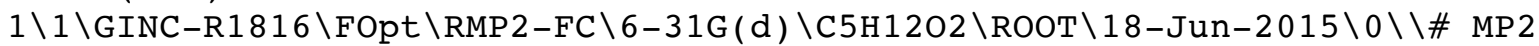
/6-31G(d) SCF=Tight INT(grid=ultrafine) OPT IOP(2/17=4) maxdisk=402653 $184 \backslash \backslash$ diMetk.MP2 $\backslash \backslash 0,1 \backslash 0,-3.6065196748,3.3996409496,1.6183245068 \backslash \mathrm{C},-2.33$ $21034365,2.9806786551,2.0901400363 \backslash 0,-3.9840728456,2.3471043417,0.6501$ $811485 \backslash \mathrm{C},-5.3470418174,1.9653626065,0.9452520866 \backslash \mathrm{C},-5.610800702,0.9236$ $500679,-0.1349693042 \backslash \mathrm{C},-6.2671296253,3.1687314775,0.7912999582 \backslash \mathrm{H},-2.01$ $50002256,3.785009268,2.758843657 \backslash \mathrm{H},-2.4006446029,2.0358324285,2.637614$ $9206 \backslash \mathrm{H},-1.6238024202,2.8816414318,1.2625436529 \backslash \mathrm{H},-7.3116011529,2.86745$ $66773,0.9199536462 \backslash \mathrm{H},-6.027548465,3.9297045427,1.5364921239 \backslash \mathrm{H},-6.14470$ $09996,3.6036267605,-0.2042360372 \backslash \mathrm{H},-6.6298983738,0.5386129929,-0.03667$ $89204 \backslash \mathrm{H},-5.4967682956,1.3682109047,-1.1269709693 \backslash \mathrm{H},-4.9100162567,0.089$ $7672964,-0.0403004273 \backslash \mathrm{C},-5.4363128261,1.3565755462,2.3380224079 \backslash \mathrm{H},-5.1$ $756310005,2.1005141623,3.0935038473 \backslash \mathrm{H},-6.4529944367,1.0021107678,2.534$ $3800347 \backslash \mathrm{H},-4.7498428427,0.5089291226,2.4211136315 \backslash \backslash$ Version=ES64L-G09Re $\mathrm{VD} .01 \backslash$ State $=1-\mathrm{A} \backslash \mathrm{HF}=-345.9375054 \backslash \mathrm{MP} 2=-346.9676755 \backslash \mathrm{RMSD}=5.783 e-09 \backslash \mathrm{RMSF}=8$ $.463 e-06 \backslash \mathrm{Dipole}=-0.0207669,-0.3292787,0.3470544 \backslash \mathrm{PG}=\mathrm{C} 01[\mathrm{X}(\mathrm{C} 5 \mathrm{H} 12 \mathrm{O} 2)] \backslash \backslash @$

17-)

Product $17-\mathrm{H}$

$\mathrm{HSCH} 2 \mathrm{CH} 2-\mathrm{H}$

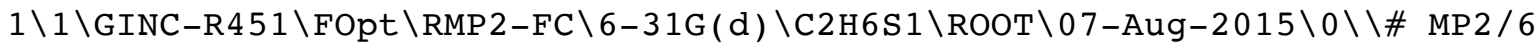
$-31 \mathrm{G}(\mathrm{d}) \mathrm{SCF}=\mathrm{Tight}$ INT(grid=ultrafine) OPT IOP(2/17=4) maxdisk=93952409 $6 \backslash \backslash$ diMetk.MP2 $\backslash \backslash 0,1 \backslash \mathrm{S},-2.7142457528,2.5247208324,1.0451253739 \backslash \mathrm{H},-2.2668$ $01231,1.2597074609,1.0335784614 \backslash \mathrm{C},-4.4727730183,2.1114088964,1.2775370$ $714 \backslash \mathrm{C},-5.0562561705,1.2606722577,0.1582784421 \backslash \mathrm{H},-4.9819005709,3.077710$ $3996,1.3274870311 \backslash \mathrm{H},-4.6068292438,1.6271871077,2.2481032068 \backslash \mathrm{H},-4.55769$ $40747,0.2887417091,0.1038356694 \backslash \mathrm{H},-4.9376601844,1.7567813662,-0.807395$ $2162 \backslash \mathrm{H},-6.1223827536,1.08348297,0.3323819602 \backslash \backslash$ Version=ES64L-G09RevD. 01 $\backslash$ State $=1-\mathrm{A} \backslash \mathrm{HF}=-476.7357055 \backslash \mathrm{MP} 2=-477.1203425 \backslash \mathrm{RMSD}=1.675 \mathrm{e}-09 \backslash \mathrm{RMSF}=1.292 \mathrm{e}$ $-05 \backslash \mathrm{Dipole}=-0.512579,-0.4993295,0.0469497 \backslash \mathrm{PG}=\mathrm{C} 01 \quad[\mathrm{X}(\mathrm{C} 2 \mathrm{H} 6 \mathrm{~S} 1)] \backslash \backslash @$

Product $17-\mathrm{CH} 3$

$\mathrm{HSCH} 2 \mathrm{CH} 2-\mathrm{CH} 3$

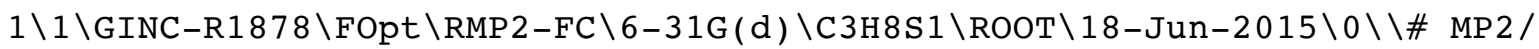
6-31G(d) SCF=Tight INT(grid=ultrafine) OPT IOP (2/17=4) maxdisk=2684354 $56 \backslash \backslash$ diMetk.MP2 $\backslash \backslash 0,1 \backslash \mathrm{S},-2.7141431704,2.5189771723,1.0338275958 \backslash \mathrm{H},-2.270$ $8416552,1.2525089096,1.030064906 \backslash \mathrm{C},-4.472917884,2.1115907329,1.2758722$ $939 \backslash \mathrm{C},-5.0732146097,1.2595934135,0.1646602672 \backslash \mathrm{H},-4.9828103876,3.078911$ $0431,1.3247645056 \backslash \mathrm{H},-4.6064223639,1.6278443329,2.24807804 \backslash \mathrm{H},-4.5341556$ $287,0.3062603059,0.1085267956 \backslash \mathrm{H},-4.9174232264,1.7635024681,-0.79468815$ $29 \backslash \mathrm{C},-6.5604048755,0.9981241603,0.3911464316 \backslash \mathrm{H},-6.7291077334,0.4756772$ $341,1.3377623358 \backslash \mathrm{H},-7.1223591684,1.936600968,0.4203787582 \backslash \mathrm{H},-6.9770892$ $967,0.3824492593,-0.4108537771 \backslash \backslash$ Version=ES64L-G09RevD .01 $\backslash$ State $=1-\mathrm{A} \backslash \mathrm{HF}=$ $-515.7706251 \backslash M P 2=-516.2866728 \backslash \mathrm{RMSD}=8.174 \mathrm{e}-09 \backslash \mathrm{RMSF}=2.809 \mathrm{e}-05 \backslash \mathrm{Dipole}=-0$. $534081,-0.5143577,0.0266342 \backslash \mathrm{PG}=\mathrm{C} 01 \quad[\mathrm{X}(\mathrm{C} 3 \mathrm{H} 8 \mathrm{~S} 1)] \backslash \backslash @$ 
$18-$ )

Product $18-\mathrm{H}$

$\mathrm{HSCH} 2 \mathrm{C}(\mathrm{CH} 3) 2-\mathrm{H}$

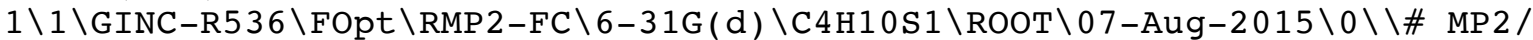
6-31G(d) SCF=Tight INT(grid=ultrafine) OPT IOP(2/17=4) maxdisk=9395240 $96 \backslash \backslash$ diMetk.MP2 $\backslash \backslash 0,1 \backslash \mathrm{S},-2.834756303,2.1032878781,0.8313309201 \backslash \mathrm{H},-2.7718$ $914056,1.1899371657,1.8125415456 \backslash \mathrm{C},-4.6534031942,2.216923031,0.7881890$ $664 \backslash \mathrm{C},-5.3823393721,0.9490063757,0.3404990342 \backslash \mathrm{H},-4.8727164036,3.032222$ $6624,0.0895458542 \backslash \mathrm{H},-5.0104094872,2.533514897,1.7729248985 \backslash \mathrm{H},-5.061511$ $9219,0.1272398305,0.996206457 \backslash \mathrm{C},-5.0525121303,0.5757361211,-1.10140296$ $31 \backslash \mathrm{H},-5.5672956471,-0.3462282494,-1.3885555196 \backslash \mathrm{H},-5.3809002831,1.36996$ $05306,-1.7816760559 \backslash \mathrm{H},-3.9791725809,0.4296094233,-1.2411714098 \backslash \mathrm{C},-6.88$ $82554882,1.1430969893,0.5206957904 \backslash \mathrm{H},-7.433868085,0.2394945985,0.23219$ $97696 \backslash \mathrm{H},-7.1419306284,1.3747671138,1.5600976129 \backslash \mathrm{H},-7.2465290694,1.9661$ $196323,-0.1074650005 \backslash \backslash$ Version=ES64L-G09RevD.01 \State $=1-A \backslash H F=-554.80436$ $98 \backslash M P 2=-555.4548861 \backslash \mathrm{RMSD}=3.725 e-09 \backslash \mathrm{RMSF}=6.308 e-06 \backslash \mathrm{Dipole}=-0.6212378,-0$ $.2829035,0.2176051 \backslash \mathrm{PG}=\mathrm{C} 01 \quad[\mathrm{X}(\mathrm{C} 4 \mathrm{H} 10 \mathrm{~S} 1)] \backslash \backslash @$

\section{Product $18-\mathrm{CH} 3$}

$\mathrm{HSCH} 2 \mathrm{C}(\mathrm{CH} 3) 2-\mathrm{CH} 3$

$1 \backslash 1 \backslash G I N C-R 2760 \backslash F O p t \backslash R M P 2-F C \backslash 6-31 G(d) \backslash C 5 H 12 S 1 \backslash R O O T \backslash 18-J u n-2015 \backslash 0 \backslash \backslash \#$ MP2 /6-31G(d) SCF=Tight INT(grid=ultrafine) OPT IOP(2/17=4) maxdisk=402653 $184 \backslash \backslash$ diMetk.MP $2 \backslash \backslash 0,1 \backslash \mathrm{S},-2.831218495,2.1329869653,0.7569912046 \backslash \mathrm{H},-2.651$ $8910104,1.4039660991,1.8675256004 \backslash \mathrm{C},-4.6548539151,2.1611906306,0.80310$ $22513 \backslash \mathrm{C},-5.3873137547,0.886049184,0.3592153741 \backslash \mathrm{H},-4.936313818,2.985881$ $648,0.1389952049 \backslash \mathrm{H},-4.9734521244,2.4418059402,1.8126788872 \backslash \mathrm{C},-5.010662$ $9008,-0.2964366051,1.2510338422 \backslash \mathrm{C},-5.0646073316,0.5611059967,-1.098593$ $5954 \backslash \mathrm{H},-5.5998118977,-1.1785369787,0.9799133558 \backslash \mathrm{H},-3.9534158185,-0.554$ $1787621,1.1411933714 \backslash \mathrm{H},-5.2064630827,-0.0723651968,2.3063642315 \backslash \mathrm{H},-5.6$ $124097383,-0.3313266142,-1.4192370829 \backslash \mathrm{H},-5.3542449524,1.3892529298,-1$. $7553666584 \backslash \mathrm{H},-3.995605955,0.3760403816,-1.2328352072 \backslash \mathrm{C},-6.8874490832,1$ $.1646919326,0.4975317663 \backslash \mathrm{H},-7.4696685575,0.2932465415,0.1799452666 \backslash \mathrm{H},-$ $7.1541926366,1.3890867717,1.5362688668 \backslash \mathrm{H},-7.1880649281,2.0157991358,-0$ $.123396679 \backslash \backslash$ Version=ES64L-G09RevD.01 \State $=1-\mathrm{A} \backslash \mathrm{HF}=-593.8372699 \backslash \mathrm{MP} 2=-59$ $4.6249455 \backslash \mathrm{RMSD}=5.640 \mathrm{e}-09 \backslash \mathrm{RMSF}=1.151 \mathrm{e}-05 \backslash \mathrm{Dipole}=-0.5784767,-0.2681917,0$ $.2746602 \backslash \mathrm{PG}=\mathrm{C} 01 \quad[\mathrm{X}(\mathrm{C} 5 \mathrm{H} 12 \mathrm{~S} 1)] \backslash \backslash @$

19-)

Product $19-\mathrm{H}$

$\mathrm{CH} 3 \mathrm{SCH} 2 \mathrm{CH} 2-\mathrm{H}$

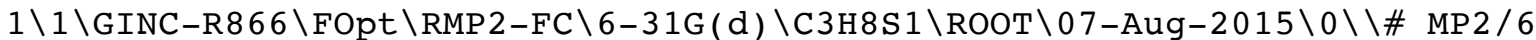
$-31 \mathrm{G}(\mathrm{d}) \mathrm{SCF}=\mathrm{Tight}$ INT(grid=ultrafine) OPT IOP(2/17=4) maxdisk=93952409 $6 \backslash \backslash$ diMetk.MP2 $\backslash \backslash 0,1 \backslash S,-2.7995438305,2.9067289449,0.2782462011 \backslash \mathrm{C},-2.5441$ $571827,2.9357633933,2.0680285052 \backslash \mathrm{C},-4.579762226,2.5586257512,0.2237924$ $781 \backslash \mathrm{C},-4.9679636088,1.1616188806,0.6920305874 \backslash \mathrm{H},-4.8633404731,2.696422$ $0793,-0.8241087535 \backslash \mathrm{H},-5.1071699504,3.3254077421,0.8017149815 \backslash \mathrm{H},-4.7153$ $648489,1.0048519207,1.7435389456 \backslash \mathrm{H},-4.4522919329,0.4010103389,0.101324$ $292 \backslash \mathrm{H},-1.5111828704,3.2443778858,2.2408430542 \backslash \mathrm{H},-3.2064710717,3.662442$ $2216,2.5457264645 \backslash \mathrm{H},-2.6899809348,1.9541752765,2.5223802623 \backslash \mathrm{H},-6.04725$ $207,1.0134275652,0.5837349815 \backslash \backslash$ Version=ES64L-G09RevD.01\State=1-A $\backslash \mathrm{HF}=-$ $515.7692248 \backslash \mathrm{MP} 2=-516.2879504 \backslash \mathrm{RMSD}=9.759 \mathrm{e}-09 \backslash \mathrm{RMSF}=7.721 \mathrm{e}-06 \backslash \mathrm{Dipole}=-0.4$ $69602,-0.1207397,0.5048752 \backslash \mathrm{PG}=\mathrm{C} 01 \quad[\mathrm{X}(\mathrm{C} 3 \mathrm{H} 8 \mathrm{~S} 1)] \backslash \backslash @$

Product $19-\mathrm{CH} 3$

CH3SCH $2 \mathrm{CH} 2-\mathrm{CH} 3$

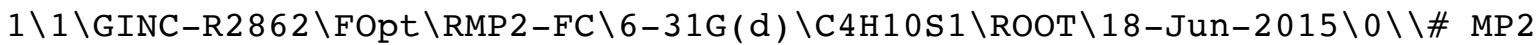
/6-31G(d) SCF=Tight INT(grid=ultrafine) OPT IOP(2/17=4) maxdisk=134217 $728 \backslash \backslash$ diMetk.MP2 $\backslash \backslash 0,1 \backslash S,-2.7935540484,2.8839444724,0.2816767216 \backslash \mathrm{C},-2.54$ $45049637,2.9399817853,2.0720068008 \backslash \mathrm{C},-4.5768948768,2.5531379022,0.2266$ 
$779256 \backslash \mathrm{C},-4.9878099976,1.157501339,0.6835666727 \backslash \mathrm{H},-4.8648762256,2.6991$ $482516,-0.8202201209 \backslash \mathrm{H},-5.0986480182,3.3198535956,0.8121889276 \backslash \mathrm{H},-4.68$ $10185522,1.0006907561,1.7233736816 \backslash \mathrm{H},-4.4505752696,0.4160388581,0.0829$ $33171 \backslash \mathrm{H},-1.5094716489,3.2424126211,2.2439730191 \backslash \mathrm{H},-3.2023204932,3.6804$ $646314,2.5344745926 \backslash \mathrm{H},-2.7013707359,1.9675253899,2.5419079381 \backslash \mathrm{C},-6.495$ $0098068,0.9446032503,0.5601778621 \backslash \mathrm{H},-6.7790668247,-0.0596727173,0.8872$ $017256 \backslash \mathrm{H},-7.0457804261,1.6653648354,1.1724906294 \backslash \mathrm{H},-6.8234181122,1.065$ $5150288,-0.4767295469 \backslash \backslash$ Version=ES64L-G09RevD.01 \State $=1-A \backslash H F=-554.8040$ $093 \backslash \mathrm{MP} 2=-555.4542721 \backslash \mathrm{RMSD}=6.063 \mathrm{e}-09 \backslash \mathrm{RMSF}=4.416 \mathrm{e}-06 \backslash \mathrm{Dipole}=-0.4799494,-$ $0.1380099,0.5110013 \backslash \mathrm{PG}=\mathrm{C} 01[\mathrm{X}(\mathrm{C} 4 \mathrm{H} 10 \mathrm{~S} 1)] \backslash \backslash @$

20-)

Product $20-\mathrm{H}$

$\mathrm{CH} 3 \mathrm{SCH} 2 \mathrm{C}$ ( $\mathrm{CH} 3) 2-\mathrm{CH} 3$

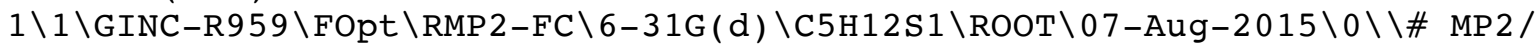
6-31G(d) SCF=Tight INT(grid=ultrafine) OPT IOP(2/17=4) maxdisk=9395240

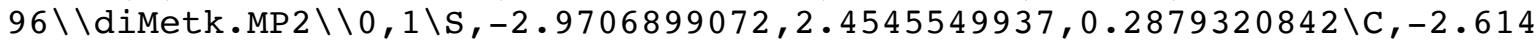
$560969,2.7739708614,2.0319390358 \backslash \mathrm{C},-4.7853942837,2.4516073417,0.348527$ $2713 \backslash \mathrm{C},-5.4350073953,1.2395175011,1.0204796473 \backslash \mathrm{H},-5.1149711532,2.50515$ $02316,-0.6962914015 \backslash \mathrm{H},-5.1129488398,3.3780687703,0.8361180006 \backslash \mathrm{H},-5.022$ $2079268,1.1423006657,2.033458309 \backslash \mathrm{C},-5.1513617173,-0.0509481377,0.25786$ $90074 \backslash \mathrm{H},-1.5333373241,2.8964324227,2.1237422674 \backslash \mathrm{H},-3.0975901906,3.6957$ $63236,2.3665951287 \backslash \mathrm{H},-2.9230385781,1.9467932037,2.6740219621 \backslash \mathrm{H},-5.6030$ $702658,-0.9084939697,0.7662998109 \backslash \mathrm{H},-5.5769685288,0.0044640037,-0.7508$ $028324 \backslash \mathrm{H},-4.0777032602,-0.2305933188,0.1650267075 \backslash \mathrm{C},-6.939987334,1.478$ $2157115,1.1455887819 \backslash \mathrm{H},-7.4290985414,0.6337741798,1.641079855 \backslash \mathrm{H},-7.155$ $9344431,2.3821723242,1.7242290268 \backslash \mathrm{H},-7.3930343416,1.5941129793,0.15468$ $23378 \backslash \backslash$ Version=ES6 4L-G09RevD.01 State $=1-\mathrm{A} \backslash \mathrm{HF}=-593.8377709 \backslash \mathrm{MP} 2=-594.622$ $8713 \backslash \mathrm{RMSD}=8.010 \mathrm{e}-09 \backslash \mathrm{RMSF}=1.571 \mathrm{e}-06 \backslash \mathrm{Dipole}=-0.4270185,0.0354181,0.55195$ $01 \backslash P G=C 01 \quad[X(C 5 H 12 S 1)] \backslash \backslash @$

\section{Product $20-\mathrm{CH} 3$}

$\mathrm{CH} 3 \mathrm{SCH} 2 \mathrm{C}(\mathrm{CH} 3) 2-\mathrm{CH} 3$

$1 \backslash 1 \backslash \mathrm{GINC}-\mathrm{R} 3296 \backslash \mathrm{FOpt} \backslash \mathrm{RMP} 2-\mathrm{FC} \backslash 6-31 \mathrm{G}(\mathrm{d}) \backslash \mathrm{C} 6 \mathrm{H} 14 \mathrm{~S} 1 \backslash \mathrm{ROOT} \backslash 18-\mathrm{Jun}-2015 \backslash 0 \backslash \backslash \# \mathrm{MP} 2$ /6-31G(d) SCF=Tight INT(grid=ultrafine) OPT IOP(2/17=4) maxdisk=402653 $184 \backslash \backslash$ diMetk.MP2 $\backslash \backslash 0,1 \backslash \mathrm{S},-3.0467453987,2.5808843122,0.1814876129 \backslash \mathrm{C},-2.66$ $92655016,2.609258486,1.9488718735 \backslash \mathrm{C},-4.8610121637,2.5564133341,0.31738$ $00491 \backslash \mathrm{C},-5.5817970102,2.5285183346,-1.0374980636 \backslash \mathrm{H},-5.154862623,1.6722$ $560528,0.8973922805 \backslash \mathrm{H},-5.1805240222,3.4463581331,0.8745222245 \backslash \mathrm{C},-5.208$ $943081,1.2704900762,-1.8244201374 \backslash \mathrm{C},-5.2452192891,3.7761113404,-1.8567$ $188419 \backslash \mathrm{H},-1.5825781571,2.6267011207,2.0537256563 \backslash \mathrm{H},-3.0804489065,3.501$ $4602355,2.427662153 \backslash \mathrm{H},-3.054023267,1.7178847956,2.4506190395 \backslash \mathrm{H},-5.8198$ $011912,3.7809781651,-2.7892303297 \backslash \mathrm{H},-5.493588663,4.6862439726,-1.29966$ $97729 \backslash \mathrm{H},-4.1825353234,3.8157798583,-2.1111509655 \backslash \mathrm{H},-5.7827820648,1.224$ $9713346,-2.7562913455 \backslash \mathrm{H},-4.1454578581,1.2550504479,-2.0781228524 \backslash \mathrm{H},-5$. $4310900285,0.3682111003,-1.2440117513 \backslash \mathrm{C},-7.0859427734,2.5104730808,-0$. $7477149945 \backslash \mathrm{H},-7.6542517753,2.4901739812,-1.6837384528 \backslash \mathrm{H},-7.3640530746$, $1.6262585659,-0.1638334972 \backslash \mathrm{H},-7.3897498277,3.4010242722,-0.1867178844 \backslash$ $\backslash$ Version=ES64L-G09RevD.01\State $=1-\mathrm{A} \backslash \mathrm{HF}=-632.8704741 \backslash \mathrm{MP} 2=-633.7920927 \backslash \mathrm{R}$ $\mathrm{MSD}=8.707 e-09 \backslash \mathrm{RMSF}=2.201 \mathrm{e}-06 \backslash \mathrm{Dipole}=-0.418467,-0.0000408,0.4636287 \backslash \mathrm{PG}=$ $\mathrm{C} 01[\mathrm{X}(\mathrm{C} 6 \mathrm{H} 14 \mathrm{~S} 1)] \backslash \backslash \mathrm{Q}$

21-)

Product $21-\mathrm{H}$

$\mathrm{CH} 3 \mathrm{SCH} 2-\mathrm{H}$

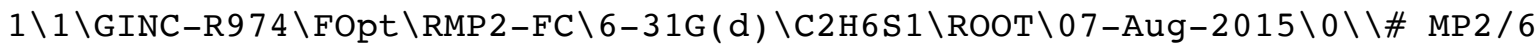
$-31 \mathrm{G}(\mathrm{d})$ SCF=Tight INT(grid=ultrafine) OPT IOP(2/17=4) maxdisk=93952409 $6 \backslash \backslash$ diMetk. MP2 $\backslash \backslash 0,1 \backslash \mathrm{H},-2.6867324458,0.7952365689,0.4683056186 \backslash \mathrm{C},-2.7452$ $901134,1.5055587766,1.296679091 \backslash \mathrm{H},-2.3896966809,1.0126553667,2.2034929$ $166 \backslash \mathrm{H},-2.097858715,2.3609256842,1.0884261514 \backslash \mathrm{S},-4.4489384988,2.0301588$ 
$931,1.5899657649 \backslash \mathrm{C},-4.7760024587,2.7894105119,-0.0164197172 \backslash \mathrm{H},-4.09783$ $69225,3.6253508057,-0.2043396765 \backslash \mathrm{H},-4.6868184801,2.0593750021,-0.82457$ $37576 \backslash \mathrm{H},-5.7998186574,3.1682319575,-0.0006456058 \backslash \backslash$ Version $=\mathrm{ES} 64 \mathrm{~L}-\mathrm{G} 09 \mathrm{ReV}$ D. $01 \backslash$ State $=1-A^{\prime} \backslash H F=-476.7348608 \backslash M P 2=-477.121108 \backslash \mathrm{RMSD}=2.460 \mathrm{e}-09 \backslash \mathrm{RMSF}=4$. $791 e-05 \backslash \mathrm{Dipole}=0.4021744,0.068614,-0.5551471 \backslash \mathrm{PG}=\mathrm{CS} \quad[\mathrm{SG}(\mathrm{C} 2 \mathrm{H} 2 \mathrm{~S} 1), \mathrm{X}(\mathrm{H} 4)] \backslash$ $\backslash$ @

\section{Product $21-\mathrm{CH} 3$}

\section{$\mathrm{CH} 3 \mathrm{SCH} 2-\mathrm{CH} 3$}

$1 \backslash 1 \backslash G I N C-R 3303 \backslash F O p t \backslash R M P 2-F C \backslash 6-31 G(d) \backslash C 3 H 8 S 1 \backslash R O O T \backslash 19-J u n-2015 \backslash 0 \backslash \backslash \#$ MP2/ 6-31G(d) SCF=Tight INT(grid=ultrafine) OPT IOP(2/17=4) maxdisk=1342177

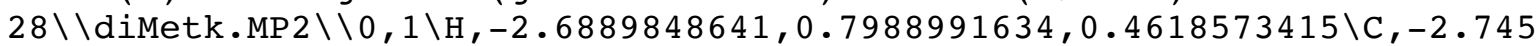
$1489489,1.5075193592,1.2917980403 \backslash \mathrm{H},-2.3840888573,1.0133097697,2.19587$ $9116 \backslash \mathrm{H},-2.100161914,2.3644302478,1.0825164175 \backslash \mathrm{S},-4.4490216295,2.027957$ $0987,1.595601498 \backslash \mathrm{C},-4.7962073552,2.7949249566,-0.0113558443 \backslash \mathrm{H},-4.07841$ $70436,3.6030794118,-0.1864055214 \backslash \mathrm{H},-4.6635557052,2.0488583974,-0.80177$ $8115 \backslash \mathrm{C},-6.2193717235,3.3393895075,-0.0332550185 \backslash \mathrm{H},-6.9464507158,2.5397$ $959668,0.1287073706 \backslash \mathrm{H},-6.3612448186,4.0942127037,0.7441382945 \backslash \mathrm{H},-6.430$ $4364243,3.8021234175,-1.0012835791 \backslash \backslash$ Version=ES64L-G0 9RevD . $01 \backslash$ State $=1-A$ $\backslash \mathrm{HF}=-515.7699225 \backslash \mathrm{MP} 2=-516.2879494 \backslash \mathrm{RMSD}=5.406 \mathrm{e}-09 \backslash \mathrm{RMSF}=5.774 \mathrm{e}-06 \backslash \mathrm{Dipole}$ $=0.3544454,0.0940976,-0.574112 \backslash \mathrm{PG}=\mathrm{C} 01[\mathrm{X}(\mathrm{C} 3 \mathrm{H} 8 \mathrm{~S} 1)] \backslash \backslash \mathrm{Q}$

$22-$ )

Product 22-H

$\mathrm{CH} 3 \mathrm{SC}(\mathrm{CH} 3) 2-\mathrm{H}$

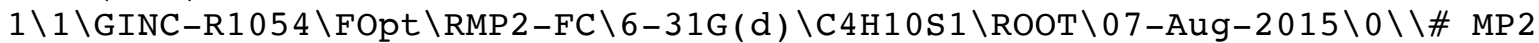
/6-31G(d) SCF=Tight INT(grid=ultrafine) OPT IOP(2/17=4) maxdisk=939524 $096 \backslash \backslash$ diMetk.MP2 $\backslash \backslash 0,1 \backslash \mathrm{H},-3.2675357998,2.7395021583,-0.2694216718 \backslash \mathrm{C},-3.1$ $169956365,2.434206246,0.7675791718 \backslash \mathrm{H},-2.257160964,1.7621602968,0.83049$ $12237 \backslash \mathrm{H},-2.901101155,3.3252189951,1.3608364291 \backslash \mathrm{S},-4.5908867922,1.66550$ $31497,1.4797722676 \backslash \mathrm{C},-4.6363238414,0.1028177392,0.5403658908 \backslash \mathrm{C},-5.7015$ $471413,-0.7825691628,1.1809840419 \backslash \mathrm{C},-4.9122666059,0.3162083219,-0.9434$ $928739 \backslash \mathrm{H},-4.932485865,-0.6485428599,-1.4623597759 \backslash \mathrm{H},-5.8808953755,0.80$ $51935657,-1.0809303 \backslash \mathrm{H},-4.1463272285,0.9337526324,-1.4194567715 \backslash \mathrm{H},-6.68$ $63600953,-0.3105045313,1.1108314699 \backslash \mathrm{H},-5.7482513924,-1.7443129853,0.66$ $09652801 \backslash \mathrm{H},-5.4825623015,-0.9691593814,2.2353776058 \backslash \mathrm{H},-3.6568138056,-0$ $.3760271844,0.6646080123 \backslash \backslash$ Version=ES64L-G09RevD. $01 \backslash$ State $=1-A \backslash H F=-554.8$ $041428 \backslash \mathrm{MP} 2=-555.4570845 \backslash \mathrm{RMSD}=4.295 e-09 \backslash \mathrm{RMSF}=1.108 \mathrm{e}-05 \backslash \mathrm{Dipole}=0.3918411$ $,-0.2728549,-0.4873445 \backslash P G=C 01[X(C 4 H 10 S 1)] \backslash \backslash @$

\section{Product22-CH3}

$\mathrm{CH} 3 \mathrm{SC}(\mathrm{CH} 3) 2-\mathrm{CH} 3$

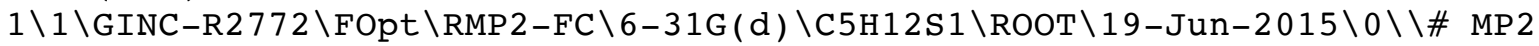
/6-31G(d) SCF=Tight INT(grid=ultrafine) OPT IOP(2/17=4) maxdisk=268435 $456 \backslash \backslash$ diMetk.MP2 $\backslash \backslash 0,1 \backslash \mathrm{H},-3.3284196742,2.7054859847,-0.2873303226 \backslash \mathrm{C},-3.1$ $277935211,2.4665500255,0.7586582142 \backslash \mathrm{H},-2.1807418052,1.9299649323,0.839$ $9312631 \backslash \mathrm{H},-3.0347485177,3.4052145785,1.3097029221 \backslash \mathrm{S},-4.4935556738,1.56$ $50836731,1.5289706383 \backslash \mathrm{C},-4.572995537,0.0151755246,0.5438398208 \backslash \mathrm{C},-5.72$ $27073575,-0.7754927757,1.1702822253 \backslash \mathrm{C},-4.8873455658,0.310907378,-0.920$ $9902327 \backslash \mathrm{H},-5.0047649906,-0.631042338,-1.4693704168 \backslash \mathrm{H},-5.8141529141,0.8$ $839087712,-1.0114251909 \backslash \mathrm{H},-4.0830317285,0.8744629566,-1.4024373853 \backslash \mathrm{H},-$ $6.6636472141,-0.2213303284,1.1025484106 \backslash \mathrm{H},-5.8437151769,-1.7260670873$, $0.6397171251 \backslash \mathrm{H},-5.5224237888,-0.9924341043,2.2237883745 \backslash \mathrm{C},-3.274237495$ $7,-0.779074408,0.6638190584 \backslash \mathrm{H},-3.3753780448,-1.7320141582,0.1314143843$ $\backslash \mathrm{H},-2.4290572014,-0.2431225034,0.2225332748 \backslash \mathrm{H},-3.0425437928,-0.9888861$ $211,1.711567837 \backslash \backslash$ Version=ES64L-G09RevD.01\State $=1-A \backslash H F=-593.8368697 \backslash M P$ $2=-594.6268912 \backslash \mathrm{RMSD}=4.191 \mathrm{e}-09 \backslash \mathrm{RMSF}=4.209 \mathrm{e}-06 \backslash \mathrm{Dipole}=0.3479658,-0.23999$ $54,-0.5192915 \backslash \mathrm{PG}=\mathrm{C} 01 \quad[\mathrm{X}(\mathrm{C} 5 \mathrm{H} 12 \mathrm{~S} 1)] \backslash \backslash @$ 
Product 23-H

$\mathrm{CH} 3 \mathrm{CH} 2 \mathrm{SCH} 2-\mathrm{H}$

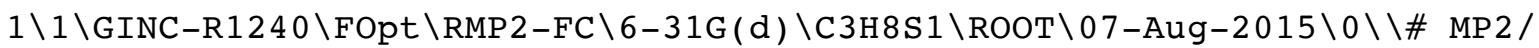
6-31G(d) SCF=Tight INT(grid=ultrafine) OPT IOP(2/17=4) maxdisk=9395240

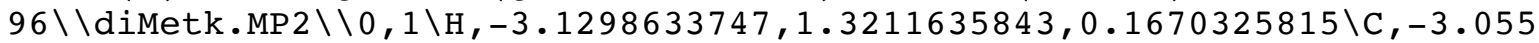
$1030617,1.646581794,1.2104005714 \backslash \mathrm{H},-2.9406111972,0.7445762996,1.819302$ $3604 \backslash \mathrm{C},-1.8575382851,2.5675998725,1.4083817358 \backslash \mathrm{S},-4.6465776519,2.35637$ $62996,1.7170651272 \backslash \mathrm{C},-4.8345203689,3.631989155,0.4493862232 \backslash \mathrm{H},-4.78130$ $4587,3.1982613465,-0.5525832562 \backslash \mathrm{H},-1.9358298656,3.4674586397,0.7934263$ $471 \backslash \mathrm{H},-1.778176581,2.8757236303,2.4534309411 \backslash \mathrm{H},-0.933894899,2.05178732$ $04,1.1267951477 \backslash \mathrm{H},-4.0889454553,4.423234587,0.5454686248 \backslash \mathrm{H},-5.82398767$ $26,4.0741994711,0.5820025958 \backslash \backslash$ Version=ES6 4L-G0 9RevD . 01 $\backslash$ State $=1-\mathrm{A} \backslash \mathrm{HF}=-5$ $15.7692252 \backslash M P 2=-516.2879504 \backslash \mathrm{RMSD}=5.361 \mathrm{e}-09 \backslash \mathrm{RMSF}=6.980 \mathrm{e}-06 \backslash \mathrm{Dipole}=0.446$ $1375,0.1679748,-0.5125876 \backslash \mathrm{PG}=\mathrm{C} 01[\mathrm{X}(\mathrm{C} 3 \mathrm{H} 8 \mathrm{~S} 1)] \backslash \backslash \mathrm{Q}$

\section{Product23-CH3}

$\mathrm{CH} 3 \mathrm{CH} 2 \mathrm{SCH} 2-\mathrm{CH} 3$

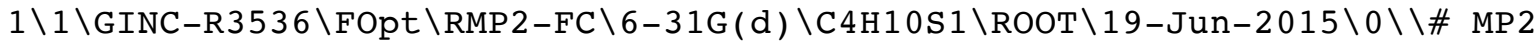
/6-31G(d) SCF=Tight INT(grid=ultrafine) OPT IOP(2/17=4) maxdisk=134217 $728 \backslash \backslash$ diMetk.MP2 $\backslash \backslash 0,1 \backslash \mathrm{H},-3.1372561295,1.3333063434,0.164027597 \backslash \mathrm{C},-3.055$ $6558753,1.6511503418,1.2091854792 \backslash \mathrm{H},-2.9399889009,0.7440119595,1.81043$ $70963 \backslash \mathrm{C},-1.8539570697,2.5671890721,1.4071603691 \backslash \mathrm{S},-4.6427509926,2.3627$ $59248,1.7294991909 \backslash \mathrm{C},-4.8566525738,3.637667555,0.4543543407 \backslash \mathrm{H},-4.74843$ $27428,3.1734795403,-0.5317826221 \backslash \mathrm{H},-1.9331765085,3.4712625331,0.798603$ $9155 \backslash \mathrm{H},-1.7687548482,2.8684696217,2.4537635568 \backslash \mathrm{H},-0.9330080123,2.05037$ $38399,1.1180773124 \backslash \mathrm{H},-4.0841017046,4.4049174236,0.5550315417 \backslash \mathrm{C},-6.2371$ $165575,4.2706748741,0.5893541727 \backslash \mathrm{H},-7.02384561,3.5231544192,0.46276044$ $23 \backslash \mathrm{H},-6.3604154961,4.7344798069,1.5713770558 \backslash \mathrm{H},-6.3719469781,5.0447434$ $215,-0.1713994483 \backslash \backslash$ Version=ES64L-G09RevD.01\State=1-A \HF=-554.8041254 MP $2=-555.4548034 \backslash \mathrm{RMSD}=2.747 \mathrm{e}-09 \backslash \mathrm{RMSF}=2.074 \mathrm{e}-05 \backslash \mathrm{Dipole}=0.3968705,0.1957$ $83,-0.5266109 \backslash \mathrm{PG}=\mathrm{C} 01 \quad[\mathrm{X}(\mathrm{C} 4 \mathrm{H} 10 \mathrm{~S} 1)] \backslash \backslash \mathrm{a}$

24-)

Product $24-\mathrm{H}$

$\mathrm{CH} 3 \mathrm{CH} 2 \mathrm{SC}(\mathrm{CH} 3) 2-\mathrm{H}$

$1 \backslash 1 \backslash G I N C-R 1240 \backslash F O p t \backslash R M P 2-F C \backslash 6-31 G(d) \backslash C 5 H 12 S 1 \backslash R O O T \backslash 07-A u g-2015 \backslash 0 \backslash \backslash \#$ MP2 $/ 6-31 \mathrm{G}(\mathrm{d}) \mathrm{SCF}=\mathrm{Tight}$ INT(grid=ultrafine) OPT IOP(2/17=4) maxdisk=939524 $096 \backslash \backslash$ diMetk.MP2 $\backslash \backslash 0,1 \backslash \mathrm{H},-2.6023925427,2.1668694371,-0.1232990054 \backslash \mathrm{C},-2.9$ $267111037,1.7897894365,0.8504744843 \backslash \mathrm{H},-3.0746080651,0.7079746839,0.764$ $0603728 \backslash \mathrm{C},-1.8661793504,2.0921865907,1.903642115 \backslash \mathrm{S},-4.5000591659,2.559$ $5408582,1.3307287593 \backslash \mathrm{C},-5.6362724158,1.8000344452,0.1219251579 \backslash \mathrm{H},-5.51$ $47704994,0.7126677307,0.2051008052 \backslash \mathrm{C},-7.0580213974,2.1685466033,0.5370$ $599345 \backslash \mathrm{H},-7.7766368856,1.7109932949,-0.1500295835 \backslash \mathrm{H},-7.279030394,1.823$ $0927644,1.5501498595 \backslash \mathrm{H},-7.1991375578,3.2532177253,0.5034021566 \backslash \mathrm{H},-1.71$ $58630917,3.1697010291,2.0095968525 \backslash \mathrm{H},-2.156587831,1.6901344931,2.87706$ $47275 \backslash \mathrm{H},-0.9114567877,1.6430539176,1.6153123897 \backslash \mathrm{C},-5.3472848524,2.2347$ $304683,-1.310452859 \backslash \mathrm{H},-4.3396633692,1.9554398657,-1.6285927495 \backslash \mathrm{H},-6.05$ $8022098,1.7597572178,-1.9959497536 \backslash \mathrm{H},-5.4473595921,3.3199754382,-1.403$ $0586639 \backslash \backslash$ Version=ES64L-G09RevD.01 \State $=1-\mathrm{A} \backslash \mathrm{HF}=-593.8389397 \backslash \mathrm{MP} 2=-594.6$ $239621 \backslash \mathrm{RMSD}=2.745 e-09 \backslash \mathrm{RMSF}=9.168 \mathrm{e}-06 \backslash \mathrm{Dipole}=0.1245265,-0.4456915,-0.48$ $28074 \backslash \mathrm{PG}=\mathrm{C} 01 \quad[\mathrm{X}(\mathrm{C} 5 \mathrm{H} 12 \mathrm{~S} 1)] \backslash \backslash \mathrm{Q}$

\section{Product $24-\mathrm{CH} 3$}

$\mathrm{CH} 3 \mathrm{CH} 2 \mathrm{SC}(\mathrm{CH} 3) 2-\mathrm{CH} 3$

$1 \backslash 1 \backslash \mathrm{GINC}-\mathrm{R} 2260 \backslash \mathrm{FOpt} \backslash \mathrm{RMP} 2-\mathrm{FC} \backslash 6-31 \mathrm{G}(\mathrm{d}) \backslash \mathrm{C} 6 \mathrm{H} 14 \mathrm{~S} 1 \backslash \mathrm{ROOT} \backslash 19-\mathrm{Jun}-2015 \backslash 0 \backslash \backslash \# \mathrm{MP} 2$ /6-31G(d) SCF=Tight INT(grid=ultrafine) OPT IOP(2/17=4) maxdisk=671088

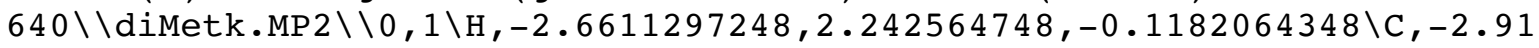
$09893121,1.7727264424,0.8367896299 \backslash \mathrm{H},-2.9162589408,0.6885148089,0.6944$ $435777 \backslash \mathrm{C},-1.8695807167,2.1500113354,1.8864812678 \backslash \mathrm{S},-4.5430431341,2.335$ $2211433,1.4020822471 \backslash \mathrm{C},-5.6902067484,1.6749330291,0.1249929435 \backslash \mathrm{C},-5.71$ 
$83312565,0.1478175787,0.1502424528 \backslash \mathrm{C},-7.0618968009,2.2223376912,0.5234$ $97486 \backslash \mathrm{H},-7.818319948,1.8599318261,-0.181217292 \backslash \mathrm{H},-7.3413865229,1.88977$ $12052,1.5278333858 \backslash \mathrm{H},-7.0686697436,3.3160784272,0.5055413295 \backslash \mathrm{H},-5.9947$ $477446,-0.2184168039,1.1425390535 \backslash \mathrm{H},-6.4542945515,-0.2191097728,-0.574$ $9539897 \backslash \mathrm{H},-4.7481462421,-0.2800190677,-0.1181039543 \backslash \mathrm{H},-1.8472751969,3$. $2311498095,2.0452825149 \backslash \mathrm{H},-2.0859238656,1.6682154901,2.8429833235 \backslash \mathrm{H},-0$ $.8754401681,1.8328529983,1.5584274703 \backslash \mathrm{C},-5.3167597545,2.1817030312,-1$. $2663157104 \backslash \mathrm{H},-4.3495147831,1.7901731223,-1.5938403419 \backslash \mathrm{H},-6.0701980603$, $1.8519879015,-1.9912670065 \backslash \mathrm{H},-5.2730027844,3.2742640559,-1.2835469528 \backslash$ $\backslash$ Version=ES64L-G09RevD.01\State $=1-A \backslash H F=-632.8714229 \backslash M P 2=-633.7936953 \backslash R$ $\mathrm{MSD}=3.535 \mathrm{e}-09 \backslash \mathrm{RMSF}=1.449 \mathrm{e}-06 \backslash \mathrm{Dipole}=0.1297842,-0.3526768,-0.5347962 \backslash \mathrm{PG}$ $=\mathrm{C} 01[\mathrm{X}(\mathrm{C} 6 \mathrm{H} 14 \mathrm{~S} 1)] \backslash \backslash @$

25-)

Product $25-\mathrm{H}$

$\mathrm{HSSCH} 2-\mathrm{H}$

$1 \backslash 1 \backslash G I N C-R 113 \backslash F O p t \backslash R M P 2-F C \backslash 6-31 G(d) \backslash C 1 H 4 S 2 \backslash R O O T \backslash 07-A u g-2015 \backslash 0 \backslash \backslash \#$ MP $2 / 6$ $-31 G(d) \quad S C F=T i g h t$ INT(grid=ultrafine) OPT IOP(2/17=4) maxdisk=13421772 $8 \backslash \backslash \mathrm{tk} . M P 2 \backslash \backslash 0,1 \backslash \mathrm{S},-0.1756708366,0.1936383778,-0.6181191409 \backslash \mathrm{C}, 1.13575364$ $67,1.2643478556,0.0290461792 \backslash \mathrm{S},-1.630647747,1.5627408802,-1.1332741202$ $\backslash \mathrm{H},-2.275819263,1.6570988923,0.0437394588 \backslash \mathrm{H}, 1.9869447242,0.6136411094$, $0.2470065631 \backslash \mathrm{H}, 1.432832938,2.006135485,-0.7130198127 \backslash \mathrm{H}, 0.8246525376,1$. $7628023996,0.9483318728 \backslash \backslash$ Version=ES64L-G09RevD . $01 \backslash$ State $=1-A \backslash H F=-835.21$ $09889 \backslash M P 2=-835.5823096 \backslash R M S D=3.118 e-09 \backslash R M S F=1.827 e-05 \backslash D i p o l e=0.3258097$, $0.3827749,0.6289306 \backslash P G=C 01[X(C 1 H 4 S 2)] \backslash \backslash @$

Product $25-\mathrm{CH} 3$

HSSCH $2-\mathrm{CH} 3$

$1 \backslash 1 \backslash G I N C-R 106 \backslash F O p t \backslash R M P 2-F C \backslash 6-31 G(d) \backslash C 2 H 6 S 2 \backslash R O O T \backslash 15-J u n-2015 \backslash 0 \backslash \backslash \#$ MP $2 / 6$ $-31 \mathrm{G}(\mathrm{d}) \mathrm{SCF}=\mathrm{Tight}$ INT(grid=ultrafine) OPT IOP(2/17=4) maxdisk=13421772 $8 \backslash \backslash \mathrm{tk} . \mathrm{MP} 2 \backslash \backslash 0,1 \backslash \mathrm{S},-0.1797317588,0.1975505632,-0.5882436825 \backslash \mathrm{C}, 1.13683243$ $9,1.2753425626,0.0588088366 \backslash \mathrm{S},-1.6262226911,1.5585333273,-1.1496560454$ $\backslash \mathrm{H},-2.3137377556,1.6543339263,0.0024659225 \backslash \mathrm{H}, 1.9895812322,0.599135059$, $0.1873143551 \backslash \mathrm{H}, 1.4034671005,2.0011787922,-0.7139563634 \backslash \mathrm{C}, 0.7927938265$, $1.9648584297,1.3690176816 \backslash \mathrm{H},-0.0578971633,2.6392294856,1.2420418801 \backslash \mathrm{H}$, $0.5438229941,1.2328085947,2.1411896739 \backslash \mathrm{H}, 1.6426187766,2.5608262594,1.7$ $155207414 \backslash \backslash$ Version=ES6 4L-G09RevD.01\State $=1-\mathrm{A} \backslash \mathrm{HF}=-874.2466417 \backslash \mathrm{MP} 2=-874$ $.7508285 \backslash \mathrm{RMSD}=2.282 \mathrm{e}-09 \backslash \mathrm{RMSF}=1.283 e-05 \backslash \mathrm{Dipole}=0.3351631,0.4172005,0.66$ $75531 \backslash \mathrm{PG}=\mathrm{C} 01 \quad[\mathrm{X}(\mathrm{C} 2 \mathrm{H} 6 \mathrm{~S} 2)] \backslash \backslash @$

26-)

Product $26-\mathrm{H}$

$\operatorname{HSSC}(\mathrm{CH} 3) 2-\mathrm{H}$

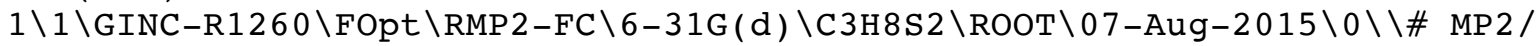
6-31G(d) SCF=Tight INT(grid=ultrafine) OPT IOP(2/17=4) maxdisk=9395240

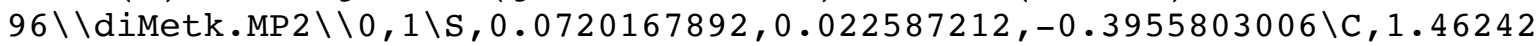
$28369,0.8756542275,0.4382671515 \backslash \mathrm{S},-0.8807161768,1.5538103356,-1.399387$ $1402 \backslash \mathrm{H},-1.7345478281,1.9482861715,-0.4371549024 \backslash \mathrm{H}, 1.0297799335,1.66659$ $86115,1.060701398 \backslash \mathrm{C}, 2.447793354,1.4767123576,-0.5521047719 \backslash \mathrm{H}, 3.2490370$ $161,1.992582735,-0.0120883952 \backslash \mathrm{H}, 2.8929077546,0.6907322618,-1.168941997$ $8 \backslash \mathrm{H}, 1.9592604785,2.2000900063,-1.2091861497 \backslash \mathrm{C}, 2.118012911,-0.170584598$ $7,1.3358750438 \backslash \mathrm{H}, 1.4142824185,-0.5708277831,2.0704287978 \backslash \mathrm{H}, 2.509141786$ $3,-1.0011686297,0.7400990434 \backslash \mathrm{H}, 2.9559827263,0.2839300927,1.8728732234 \backslash$ $\backslash$ Version=ES64L-G09RevD.01\State $=1-A \backslash H F=-913.2818151 \backslash M P 2=-913.9205449 \backslash R$ $M S D=7.710 e-09 \backslash R M S F=8.380 e-06 \backslash D i p o l e=0.3566819,0.4011597,0.6773036 \backslash P G=C$ $01[\mathrm{X}(\mathrm{C} 3 \mathrm{H} 8 \mathrm{~S} 2)] \backslash \backslash @$

Product $26-\mathrm{CH} 3$

$\operatorname{HSSC}(\mathrm{CH} 3$ ) $2-\mathrm{CH} 3$

$1 \backslash 1 \backslash G I N C-R 1980 \backslash F O p t \backslash R M P 2-F C \backslash 6-31 G(d) \backslash C 4 H 10 S 2 \backslash R O O T \backslash 15-J u n-2015 \backslash 0 \backslash \backslash \#$ MP2 
/6-31G(d) SCF=Tight INT(grid=ultrafine) OPT IOP(2/17=4) maxdisk=268435

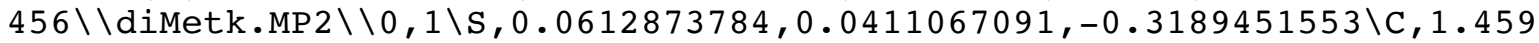
$2308771,0.9225037702,0.5031046107 \backslash \mathrm{S},-0.8619922707,1.4889305138,-1.4626$ $044262 \backslash \mathrm{H},-1.7482669399,1.9569977474,-0.5651731804 \backslash \mathrm{C}, 0.9395815637,2.027$ $3896343,1.4148363857 \backslash \mathrm{C}, 2.4250533868,1.4814310057,-0.5350563057 \backslash \mathrm{H}, 3.266$ $2308433,1.9686597054,-0.0289025786 \backslash \mathrm{H}, 2.816222273,0.6836115844,-1.17231$ $90755 \backslash \mathrm{H}, 1.9328093972,2.2239483094,-1.1690193063 \backslash \mathrm{H}, 1.7798718921,2.49776$ $36125,1.937655243 \backslash \mathrm{H}, 0.4269884369,2.8035822314,0.8391999461 \backslash \mathrm{H}, 0.2466577$ $683,1.6269649355,2.1606373262 \backslash \mathrm{C}, 2.1317554342,-0.1783367912,1.325262749$ $5 \backslash \mathrm{H}, 1.4466031381,-0.5953858336,2.0696335623 \backslash \mathrm{H}, 2.4889737249,-0.99023984$ $, 0.6844521542 \backslash \mathrm{H}, 2.9933530966,0.2450927056,1.8521150503 \backslash \backslash$ Version=ES6 $4 \mathrm{~L}-$ G09RevD.01\State $=1-A \backslash H F=-952.3156567 \backslash M P 2=-953.0920416 \backslash$ RMSD $=8.163 e-09 \backslash R$ $\mathrm{MSF}=3.000 \mathrm{e}-05 \backslash \mathrm{Dipole}=0.3573361,0.4593842,0.6663588 \backslash \mathrm{PG}=\mathrm{C} 01 \quad[\mathrm{X}(\mathrm{C} 4 \mathrm{H} 10 \mathrm{~S} 2)]$ $\backslash \backslash$ Q

27-)

Product $27-\mathrm{H}$

$\mathrm{CH} 3 \mathrm{SSCH} 2-\mathrm{H}$

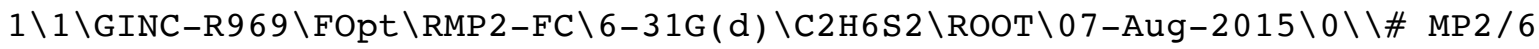
$-31 \mathrm{G}(\mathrm{d}) \mathrm{SCF}=\mathrm{Tight}$ INT(grid=ultrafine) OPT IOP(2/17=4) maxdisk=53687091 $2 \backslash \backslash t \mathrm{k} . M P 2 \backslash \backslash 0,1 \backslash \mathrm{S},-0.1231600482,-0.0708382276,-0.6579380917 \backslash \mathrm{C}, 1.1861450$ $23,1.0423823606,-0.0769011087 \backslash S,-1.673853825,1.2314500486,-1.008492809$ $\backslash \mathrm{C},-1.3177089078,1.8040537707,-2.6926482305 \backslash \mathrm{H}, 1.4844969461,1.747817922$ $4,-0.8543336665 \backslash \mathrm{H}, 2.0437298602,0.4129767642,0.1754713991 \backslash \mathrm{H},-2.13246686$ $64,2.4766298675,-2.973824018 \backslash \mathrm{H},-0.376457186,2.3546985569,-2.7364447536$ $\backslash \mathrm{H},-1.2881705537,0.9649276075,-3.3885342175 \backslash \mathrm{H}, 0.8653105578,1.585874329$ $1,0.8124574965 \backslash \backslash$ Version=ES64L-G09RevD. $01 \backslash$ State $=1-A \backslash H F=-874.2471713 \backslash \mathrm{MP} 2$ $=-874.7532122 \backslash \mathrm{RMSD}=8.450 \mathrm{e}-09 \backslash \mathrm{RMSF}=1.314 \mathrm{e}-05 \backslash \mathrm{Dipole}=0.5778988,0.5851111$ ,$-0.38281 \backslash \mathrm{PG}=\mathrm{C} 01[\mathrm{X}(\mathrm{C} 2 \mathrm{H} 6 \mathrm{~S} 2)] \backslash \backslash @$

Product $27-\mathrm{CH} 3$

$\mathrm{CH} 3 \mathrm{SSCH} 2-\mathrm{CH} 3$

$1 \backslash 1 \backslash G I N C-R 1979 \backslash F O p t \backslash R M P 2-F C \backslash 6-31 G(d) \backslash C 3 H 8 S 2 \backslash R O O T \backslash 15-J u n-2015 \backslash 0 \backslash \backslash \#$ MP $/$ 6-31G(d) SCF=Tight INT(grid=ultrafine) OPT IOP (2/17=4) maxdisk=2684354 $56 \backslash \backslash t \mathrm{k} . M P 2 \backslash \backslash 0,1 \backslash \mathrm{S},-0.1494382138,-0.0437816024,-0.6315656562 \backslash \mathrm{C}, 1.173966$ $6886,1.0581437325,-0.0336294074 \backslash \mathrm{S},-1.6842128782,1.2690592108,-1.017911$ $2108 \backslash \mathrm{C},-1.3095284224,1.7916106565,-2.7144491713 \backslash \mathrm{H}, 1.3755935122,1.81734$ $37522,-0.7952715543 \backslash \mathrm{H}, 2.0561572443,0.4103891805,0.0209591547 \backslash \mathrm{H},-2.1101$ $912634,2.4714406903,-3.0180690964 \backslash \mathrm{H},-0.3577023565,2.323168675,-2.76632$ $97402 \backslash \mathrm{H},-1.2907345097,0.934064352,-3.3879884167 \backslash \mathrm{C}, 0.8836836036,1.69450$ $17085,1.3163747049 \backslash \mathrm{H}, 0.728869753,0.9281252688,2.0793817258 \backslash \mathrm{H}, 1.7223758$ $886,2.3276066419,1.6229840606 \backslash \mathrm{H},-0.0128390464,2.3167117335,1.265473607$ $3 \backslash \backslash$ Version=ES64L-G09RevD. $01 \backslash$ State $=1-A \backslash H F=-913.2826301 \backslash M P 2=-913.9217339$ $\backslash \mathrm{RMSD}=8.403 \mathrm{e}-09 \backslash \mathrm{RMSF}=7.639 \mathrm{e}-06 \backslash \mathrm{Dipole}=0.589956,0.5945587,-0.3356349 \backslash \mathrm{PG}$ $=\mathrm{C} 01[\mathrm{X}(\mathrm{C} 3 \mathrm{H} 8 \mathrm{~S} 2)] \backslash \backslash \mathrm{Q}$

28-)

Product $28-\mathrm{H}$

$\mathrm{CH} 3 \mathrm{SSC}$ ( $\mathrm{CH} 3$ ) $2-\mathrm{H}$

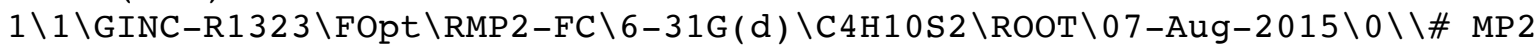
/6-31G(d) SCF=Tight INT(grid=ultrafine) OPT IOP(2/17=4) maxdisk=939524 $096 \backslash \backslash t \mathrm{k} . \mathrm{MP} 2 \backslash \backslash 0,1 \backslash \mathrm{S}, 0.3536607055,-0.1119646609,-0.9418348874 \backslash \mathrm{C}, 1.588098$ $3047,0.7749629397,0.0836454393 \backslash \mathrm{S},-1.121689167,1.2926148281,-1.21503425$ $07 \backslash \mathrm{C},-2.1401868966,1.0570376006,0.2679566976 \backslash \mathrm{H}, 1.060893965,1.161384267$ $7,0.9633300326 \backslash \mathrm{C}, 2.2401765724,1.9230992786,-0.6714958885 \backslash \mathrm{H}, 2.950052087$ $2,2.4431615421,-0.0189170723 \backslash \mathrm{H}, 2.781367238,1.5444284025,-1.5434085176 \backslash$ $\mathrm{H}, 1.4952707445,2.6462808495,-1.0120301051 \backslash \mathrm{H},-2.9847246835,1.7462557859$ $, 0.1827231637 \backslash \mathrm{H},-2.5145191131,0.0340602262,0.3209863064 \backslash \mathrm{H},-1.581899876$ $8,1.293768145,1.1753678636 \backslash \mathrm{C}, 2.6062150658,-0.2709038736,0.5292760465 \backslash \mathrm{H}$ , $2.1360770647,-1.0708977995,1.1071445925 \backslash \mathrm{H}, 3.1064836744,-0.7161760928$, 
$-0.3364324467 \backslash \mathrm{H}, 3.3697963149,0.2038175608,1.1530760261 \backslash \backslash$ Version=ES64LG09RevD.01 State $=1-\mathrm{A} \backslash \mathrm{HF}=-952.3178672 \backslash \mathrm{MP} 2=-953.0916358 \backslash \mathrm{RMSD}=5.825 \mathrm{e}-09 \backslash \mathrm{R}$ $\mathrm{MSF}=4.756 \mathrm{e}-06 \backslash \mathrm{Dipole}=0.1456845,0.2414369,0.8889021 \backslash \mathrm{PG}=\mathrm{C} 01$ [X(C4H10S2)] $\backslash \backslash @$

Product $28-\mathrm{CH} 3$

$\mathrm{CH} 3 \mathrm{SSC}$ ( $\mathrm{CH} 3$ ) $2-\mathrm{CH} 3$

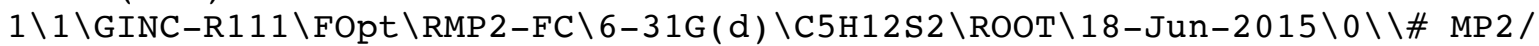
6-31G(d) SCF=Tight INT(grid=ultrafine) OPT IOP(2/17=4) maxdisk=6710886 $40 \backslash \backslash \mathrm{tk} . \mathrm{MP} 2 \backslash \backslash 0,1 \backslash \mathrm{S}, 0.344644877,-0.0747891886,-0.7602967061 \backslash \mathrm{C}, 1.65445189$ $32,0.8116281712,0.1950476424 \backslash \mathrm{S},-1.099771499,1.3410314097,-1.1264366393$ $\backslash \mathrm{C},-2.2854006107,1.0604260397,0.2195712542 \backslash \mathrm{C}, 1.0972606242,1.3919946212$ $, 1.4892809091 \backslash \mathrm{C}, 2.2786563466,1.9083091939,-0.6609976865 \backslash \mathrm{H}, 3.0791167174$ , $2.4036951551,-0.0992012775 \backslash \mathrm{H}, 2.7024156105,1.4921530885,-1.5790303623 \backslash$ $\mathrm{H}, 1.5341936465,2.6623743936,-0.931862552 \backslash \mathrm{H}, 1.9096161898,1.8509631373,2$ $.064470112 \backslash \mathrm{H}, 0.3557413389,2.1682494501,1.2797556078 \backslash \mathrm{H}, 0.6347396354,0.6$ $135242399,2.1030663472 \backslash \mathrm{H},-3.1413662221,1.7104363537,0.0175611378 \backslash \mathrm{H},-2$. $6201511642,0.0224348281,0.2244698583 \backslash \mathrm{H},-1.8630421516,1.3169253481,1.19$ $13826492 \backslash \mathrm{C}, 2.6786369957,-0.2833384166,0.495945664 \backslash \mathrm{H}, 2.2443265507,-1.07$ $60262933,1.1126034574 \backslash \mathrm{H}, 3.0649863018,-0.7294602354,-0.425661847 \backslash \mathrm{H}, 3.52$ $11149199,0.1545787037,1.0420034312 \backslash \backslash$ Version=ES64L-G0 9RevD. $01 \backslash$ State $=1-A$ $\backslash \mathrm{HF}=-991.3508872 \backslash \mathrm{MP} 2=-992.2627249 \backslash \mathrm{RMSD}=6.060 \mathrm{e}-09 \backslash \mathrm{RMSF}=6.579 \mathrm{e}-06 \backslash \mathrm{Dipole}$ $=0.1330631,0.2428113,0.8410704 \backslash \mathrm{PG}=\mathrm{C} 01[\mathrm{X}(\mathrm{C} 5 \mathrm{H} 12 \mathrm{~S} 2)] \backslash \backslash @$

\section{R-Cl Geometries}

\section{R-Cl Reactants}

$\mathrm{CH} 3-\mathrm{Cl}$

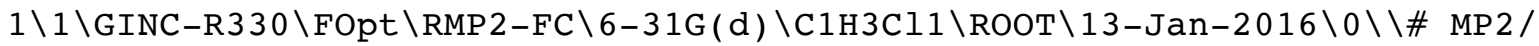
6-31G(d) SCF=Tight INT(grid=ultrafine) OPT IOP(2/17=4) maxdisk=1342177 $28 \backslash \backslash$ diMetk.MP $2 \backslash \backslash 0,1 \backslash \mathrm{Cl}, 0 ., 0.0515848487,-0.0103390195 \backslash \mathrm{C}, 0 .,-0.062681930$ $6,1.7645724151 \backslash \mathrm{H}, 0.0000004381,-1.1129671525,2.0502015005 \backslash \mathrm{H}, 0.891802388$ $8,0.4284895671,2.1494387308 \backslash \mathrm{H},-0.8918028269,0.4284888098,2.149438682 \backslash \backslash$ Version=ES64L-G09RevD.01 \State $=1-\mathrm{A} 1 \backslash \mathrm{HF}=-499.0929038 \backslash \mathrm{MP} 2=-499.3545601 \backslash \mathrm{R}$ $\mathrm{MSD}=3.633 e-09 \backslash \mathrm{RMSF}=2.282 \mathrm{e}-05 \backslash \mathrm{Dipole}=0,,-0.0517451,0.8037591 \backslash \mathrm{PG}=\mathrm{C} 03 \mathrm{~V}[\mathrm{C}$ $3(\mathrm{C} 1 \mathrm{Cl} 1), 3 \mathrm{SGV}(\mathrm{H} 1)] \backslash \backslash @$

\section{$\mathrm{CH} 3 \mathrm{CH} 2-\mathrm{Cl}$}

$1 \backslash 1 \backslash G I N C-R 158 \backslash F O p t \backslash R M P 2-F C \backslash 6-31 G(d) \backslash C 2 H 5 C 11 \backslash R O O T \backslash 13-J a n-2016 \backslash 0 \backslash \backslash \#$ MP2/ 6-31G(d) SCF=Tight INT(grid=ultrafine) OPT IOP(2/17=4) maxdisk=1342177 $28 \backslash \backslash$ dimetk.MP2 $\backslash \backslash 0,1 \backslash \mathrm{C},-2.7637157058,1.2159904932,0.0073623268 \backslash \mathrm{C},-1.561$ $4590651,2.1393549352,-0.0141476905 \backslash C l,-0.0289992044,1.2139175251,-0.00$ $01786928 \backslash \mathrm{H},-3.6806649001,1.8131943339,-0.0018517119 \backslash \mathrm{H},-2.7614892241,0$. $5957374317,0.9058278018 \backslash \mathrm{H},-2.7664014907,0.5608583092,-0.8659953521 \backslash \mathrm{H},-$ $1.5433367817,2.792498401,0.8591752041 \backslash \mathrm{H},-1.5482488756,2.7576205038,-0$. $9125857016 \backslash \backslash$ Version=ES64L-G09RevD.01\State $=1-A^{\prime} \backslash H F=-538.1311478 \backslash M P 2=-5$ $38.5240223 \backslash \mathrm{RMSD}=2.345 \mathrm{e}-09 \backslash \mathrm{RMSF}=5.676 \mathrm{e}-05 \backslash \mathrm{Dipole}=-0.7635359,0.3783297,-$ $0.0053307 \backslash \mathrm{PG}=\mathrm{CS} \quad[\mathrm{SG}(\mathrm{C} 2 \mathrm{H} 1 \mathrm{C} 11), \mathrm{X}(\mathrm{H} 4)] \backslash \backslash @$

\section{$\mathrm{HO}-\mathrm{Cl}$}

$1 \backslash 1 \backslash G I N C-R 104 \backslash F O p t \backslash R M P 2-F C \backslash 6-31 G(d) \backslash C l 1 H 101 \backslash R O O T \backslash 13-J a n-2016 \backslash 0 \backslash \backslash \#$ MP $/$ 6-31G(d) SCF=Tight INT(grid=ultrafine) OPT IOP(2/17=4) maxdisk=1342177

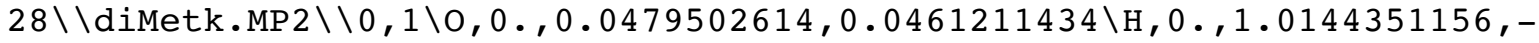
$0.1060839256 \backslash \mathrm{Cl}, 0 .,-0.0623853771,1.7599627822 \backslash \backslash$ Version=ES64L-G09RevD.0 $1 \backslash$ State $=1-A^{\prime} \backslash H F=-534.8400946 \backslash M P 2=-535.1568308 \backslash R M S D=2.762 e-09 \backslash R M S F=4.85$ $9 e-06 \backslash \mathrm{Dipole}=0,0.7065343,-0.0058414 \backslash \mathrm{PG}=\mathrm{CS} \quad[\mathrm{SG}(\mathrm{Cl} 1 \mathrm{H} 1 \mathrm{O} 1)] \backslash \backslash @$

CH3O-Cl 


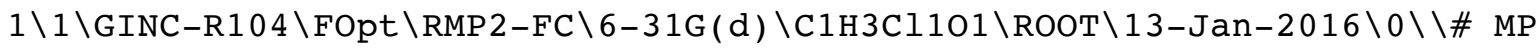
2/6-31G(d) SCF=Tight INT(grid=ultrafine) OPT IOP $(2 / 17=4$ ) maxdisk=13421 $7728 \backslash \backslash$ diMetk.MP $2 \backslash \backslash 0,1 \backslash C,-2.7267741985,2.4856790947,-1.0907877236 \backslash 0,-1$. $3431208233,2.3217754826,-1.4290308491 \backslash \mathrm{H},-2.949317977,3.519900512,-0.81$ $28659695 \backslash \mathrm{H},-3.0230762539,1.8079438933,-0.2850208446 \backslash \mathrm{H},-3.2464464725,2$. $2234486978,-2.0138940896 \backslash \mathrm{Cl},-0.37329872,2.7015506591,-0.0617907246 \backslash \backslash \mathrm{Ve}$ rsion=ES64L-G09RevD. $01 \backslash$ State $=1-A^{\prime} \backslash \mathrm{HF}=-573.869753 \backslash \mathrm{MP} 2=-574.315233 \backslash \mathrm{RMSD}=$ $9.551 \mathrm{e}-09 \backslash \mathrm{RMSF}=3.280 \mathrm{e}-05 \backslash \mathrm{Dipole}=-0.7197284,0.0939637,0.2041808 \backslash \mathrm{PG}=\mathrm{CS}$ [ $\mathrm{SG}(\mathrm{C} 1 \mathrm{H} 1 \mathrm{Cl} 1 \mathrm{O} 1), \mathrm{X}(\mathrm{H} 2)] \backslash \backslash @$

\section{$\mathrm{HS}-\mathrm{Cl}$}

$1 \backslash 1 \backslash$ GINC-R4 17 FOpt $\backslash R M P 2-F C \backslash 6-31 G(d) \backslash C l 1 H 1 S 1 \backslash R O O T \backslash 15-O c t-2015 \backslash 0 \backslash \backslash \#$ MP2/ 6-31G(d) SCF=Tight INT(grid=ultrafine) OPT IOP(2/17=4) maxdisk=1342177

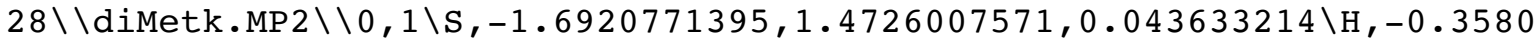
$954674,1.5986019691,-0.0316158959 \backslash \mathrm{Cl},-2.1395843932,3.1876582738,-0.980$ $6023181 \backslash \backslash$ Version=ES64L-G09RevD.01\State $=1-A^{\prime} \backslash \mathrm{HF}=-857.5498212 \backslash \mathrm{MP} 2=-857$. $7966301 \backslash \mathrm{RMSD}=6.821 \mathrm{e}-09 \backslash \mathrm{RMSF}=1.300 \mathrm{e}-04 \backslash \mathrm{Dipole}=0.5370263,-0.2616297,0.15$ $62454 \backslash P G=C S \quad[S G(C l 1 H 1 S 1)] \backslash \backslash @$

\section{$\mathrm{CH} 3 \mathrm{~S}-\mathrm{Cl}$}

$1 \backslash 1 \backslash$ GINC-R2 49 $\backslash$ FOpt $\backslash R M P 2-F C \backslash 6-31 G(d) \backslash C 1 H 3 C 11 S 1 \backslash R O O T \backslash 15-O C t-2015 \backslash 0 \backslash \backslash \#$ MP 2/6-31G(d) SCF=Tight INT(grid=ultrafine) OPT IOP $(2 / 17=4)$ maxdisk=13421 $7728 \backslash \backslash$ tk.MP $2 \backslash \backslash 0,1 \backslash S,-2.2911587527,2.626170607,-1.4287332335 \backslash \mathrm{Cl},-1.2766$ $520529,1.1120459031,-0.4916155726 \backslash \mathrm{C},-3.3883248459,3.1161707931,-0.0867$ $084342 \backslash \mathrm{H},-4.047211809,2.2969845345,0.2034873445 \backslash \mathrm{H},-2.8229319548,3.4713$ $758484,0.7755977723 \backslash \mathrm{H},-3.9914061283,3.9381838201,-0.4835281448 \backslash \backslash$ Versio $\mathrm{n}=\mathrm{ES} 64 \mathrm{~L}-\mathrm{G} 09$ RevD. $01 \backslash$ State $^{2}=1-\mathrm{A}^{\prime} \backslash \mathrm{HF}=-896.5902603 \backslash \mathrm{MP} 2=-896.9704192 \backslash \mathrm{RMSD}=8$. $823 e-09 \backslash \mathrm{RMSF}=1.508 \mathrm{e}-05 \backslash \mathrm{Dipole}=-0.6537062,0.5187967,0.3339372 \backslash \mathrm{PG}=\mathrm{CS} \quad[\mathrm{SG}$ $(\mathrm{C} 1 \mathrm{H} 1 \mathrm{C} 11 \mathrm{~S} 1), \mathrm{X}(\mathrm{H} 2) \mathrm{]} \backslash \backslash @$

\section{R-Cl Products}

Product 1-Cl) $\mathrm{CH} 3 \mathrm{CH} 2 \mathrm{CH} 2-\mathrm{Cl}$

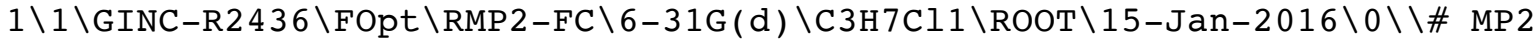
/6-31G(d) SCF=Tight INT (grid=ultrafine) OPT IOP $(2 / 17=4)$ maxdisk=939524 $096 \backslash \backslash d i M e t k . M P 2 \backslash \backslash 0,1 \backslash \mathrm{H},-2.4160686402,2.3511196943,0.2907993503 \backslash \mathrm{C},-2.96$ $87721902,2.1681374126,1.2171881162 \backslash \mathrm{H},-2.3537304757,1.5281382328,1.8569$ $416738 \backslash \mathrm{H},-3.0954148282,3.1271701563,1.726184538 \backslash \mathrm{C},-4.3238741765,1.5240$ $315355,0.9326592973 \backslash \mathrm{C},-4.1544163874,0.1915247104,0.2257043256 \backslash \mathrm{H},-4.935$ $171955,2.1845063614,0.3097819087 \backslash \mathrm{H},-4.8732241634,1.3666785591,1.866116$ $7648 \backslash \mathrm{H},-3.5822239092,-0.5087650677,0.837500375 \backslash \mathrm{H},-3.6446878432,0.31587$ $67885,-0.7318016969 \backslash \mathrm{Cl},-5.7320919371,-0.5813543819,-0.117633415 \backslash \backslash$ Versi on=ES6 4L-G0 9RevD. 0 $\backslash$ State $=1-A^{\prime} \backslash \mathrm{HF}=-577.166084 \backslash \mathrm{MP} 2=-577.6901524 \backslash \mathrm{RMSD}=6$. $884 \mathrm{e}-09 \backslash \mathrm{RMSF}=3.317 \mathrm{e}-05 \backslash \mathrm{Dipole}=0.7230083,0.4650666,0.2156063 \backslash \mathrm{PG}=\mathrm{CS} \quad[\mathrm{SG}($ $\mathrm{C} 3 \mathrm{H} 1 \mathrm{Cl} 1), \mathrm{X}(\mathrm{H} 6) \mathrm{J} \backslash \backslash @$

Product 2-Cl) $\mathrm{CH} 3 \mathrm{CH} 2 \mathrm{C}(\mathrm{CH} 3) 2-\mathrm{Cl}$

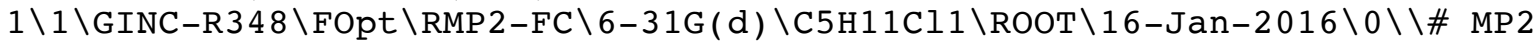
16-31G(d) SCF=Tight INT (grid=ultrafine) OPT IOP $(2 / 17=4)$ maxdisk=671088 $640 \backslash \backslash$ diMetk.MP $2 \backslash \backslash 0,1 \backslash \mathrm{H},-2.3256272436,2.3797727627,0.426610487 \backslash \mathrm{C},-2.962$ $5314515,2.2547821852,1.3051103771 \backslash \mathrm{H},-2.404530521,1.6953721747,2.059909$ $5067 \backslash \mathrm{H},-3.1699070343,3.2465810446,1.7167905312 \backslash \mathrm{C},-4.2706705812,1.56194$ $2817,0.9379024939 \backslash \mathrm{C},-4.1459708624,0.1936230904,0.2636201222 \backslash \mathrm{H},-4.85839$ $88683,2.2096535692,0.2776040199 \backslash \mathrm{H},-4.8716409712,1.4096121783,1.8447458$ $345 \backslash \mathrm{C},-3.3067729652,-0.7854289752,1.0723644546 \backslash \mathrm{Cl},-3.3267638899,0.4201$ $213703,-1.344040892 \backslash \mathrm{C},-5.527852238,-0.3817361019,-0.0174544484 \backslash \mathrm{H},-3.27$ $91914732,-1.7606118431,0.5796244483 \backslash \mathrm{H},-2.2806707651,-0.4333668573,1.19$ $07632925 \backslash \mathrm{H},-3.7526472991,-0.9093285546,2.065630365 \backslash \mathrm{H},-5.4512543119,-1$. $3324245698,-0.5510740043 \backslash \mathrm{H},-6.048944066,-0.5541682392,0.9304631186 \backslash \mathrm{H},-$ $6.1211284579,0.3093539488,-0.6216557067 \backslash \backslash$ Version=ES64L-G09RevD.01\Stat 
$\mathrm{e}=1-\mathrm{A} \backslash \mathrm{HF}=-655.2370527 \backslash \mathrm{MP} 2=-656.0320869 \backslash \mathrm{RMSD}=9.738 \mathrm{e}-09 \backslash \mathrm{RMSF}=1.703 \mathrm{e}-05 \backslash \mathrm{D}$ ipole $=-0.3701627,-0.0738096,0.7726466 \backslash P G=C 01[\mathrm{X}(\mathrm{C} 5 \mathrm{H} 11 \mathrm{Cl} 1)] \backslash \backslash @$

Product $3-\mathrm{Cl}) \mathrm{CH} 3 \mathrm{CH} 2 \mathrm{CH} 2 \mathrm{CH} 2-\mathrm{Cl}$

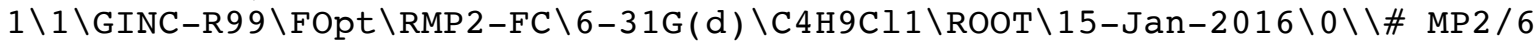
$-31 \mathrm{G}(\mathrm{d})$ SCF=Tight INT(grid=ultrafine) OPT IOP(2/17=4) maxdisk=12079595 $52 \backslash \backslash$ diMetk.MP $2 \backslash \backslash 0,1 \backslash H,-2.4518530281,2.3177661901,0.2756169469 \backslash \mathrm{C},-3.005$ $6614807,2.1350806386,1.2012855343 \backslash \mathrm{H},-2.3895724424,1.4955415507,1.84031$ $93352 \backslash \mathrm{H},-3.1367179602,3.0940250089,1.7104110839 \backslash \mathrm{C},-4.3507353936,1.4751$ $402646,0.9080366403 \backslash \mathrm{C},-4.1922879462,0.132245722,0.1960615138 \backslash \mathrm{H},-4.9619$ $834009,2.1453197036,0.2911387497 \backslash \mathrm{H},-4.9000908208,1.3282174916,1.846093$ $1033 \backslash \mathrm{H},-3.5908732379,-0.5473008146,0.8101346104 \backslash \mathrm{H},-3.6528721124,0.2712$ $04689,-0.7474902222 \backslash \mathrm{C},-5.5381502631,-0.5091725409,-0.0874229429 \backslash \mathrm{H},-6.0$ $870228714,-0.6994538525,0.8369490392 \backslash \mathrm{H},-6.149483597,0.1251489825,-0.73$ $22790811 \backslash \mathrm{Cl},-5.3598734403,-2.0836591683,-0.9218850708 \backslash \backslash$ Version=ES $64 \mathrm{~L}-\mathrm{G}$ 09 RevD.01 $\backslash$ State $=1-A^{\prime} \backslash H F=-616.2006693 \backslash M P 2=-616.8558476 \backslash$ RMSD $=6.777 \mathrm{e}-09 \backslash \mathrm{R}$ $\mathrm{MSF}=3.820 \mathrm{e}-05 \backslash \mathrm{Dipole}=0.0284276,0.8099413,0.424479 \backslash \mathrm{PG}=\mathrm{CS} \quad[\mathrm{SG}(\mathrm{C} 4 \mathrm{H} 1 \mathrm{Cl} 1), \mathrm{X}$ ( $\mathrm{H} 8$ ) $] \backslash \backslash @$

Product $4-\mathrm{Cl}) \mathrm{CH} 3 \mathrm{CH} 2 \mathrm{CH} 2 \mathrm{C}(\mathrm{CH} 3) 2-\mathrm{Cl}$

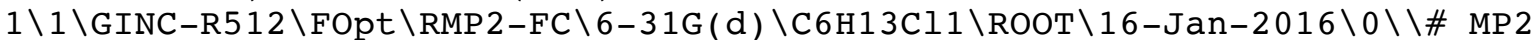
/6-31G(d) SCF=Tight INT(grid=ultrafine) OPT IOP $(2 / 17=4)$ maxdisk=671088 $640 \backslash \backslash$ diMetk.MP $2 \backslash \backslash 0,1 \backslash \mathrm{H},-2.2964484258,2.2926732122,0.4440645641 \backslash \mathrm{C},-2.92$ $71223683,2.2114618125,1.3351230338 \backslash \mathrm{H},-2.3795396056,1.5977564431,2.0585$ $245691 \backslash \mathrm{C},-3.1616908491,3.5986754426,1.9293085169 \backslash \mathrm{C},-4.2429522809,1.532$ $7337699,0.9647115169 \backslash \mathrm{C},-4.1467253098,0.1780531293,0.2598611035 \backslash \mathrm{H},-4.83$ $03537287,2.2050119943,0.3267799489 \backslash \mathrm{H},-4.8337838004,1.3727403673,1.8783$ $395721 \backslash \mathrm{C},-3.3001098404,-0.8266405671,1.0286111714 \backslash \mathrm{Cl},-3.3615149954,0.4$ $302579507,-1.3612650212 \backslash \mathrm{C},-5.5402515278,-0.3779606018,-0.0030150016 \backslash \mathrm{H}$, $-3.2965132519,-1.792335435,0.5165317643 \backslash \mathrm{H},-2.2673968707,-0.4885745171$, $1.128795316 \backslash \mathrm{H},-3.7233509403,-0.9646346968,2.0299152083 \backslash \mathrm{H},-3.6706061709$ , 4.2491383938,1.2114427729\H, $-3.7828317474,3.5413396972,2.8288361846 \backslash \mathrm{H}$ $,-2.2167926322,4.0761408292,2.2040162239 \backslash \mathrm{H},-5.4847109591,-1.3170450913$ $,-0.559498134 \backslash \mathrm{H},-6.0416184621,-0.5668555356,0.9523720759 \backslash \mathrm{H},-6.13985723$ $34,0.3323224025,-0.5780183858 \backslash \backslash$ Version=ES64L-G09RevD.01 $\backslash$ State $=1-\mathrm{A} \backslash \mathrm{HF}=-$ $694.2713134 \backslash M P 2=-695.1978388 \backslash \mathrm{RMSD}=8.564 \mathrm{e}-09 \backslash \mathrm{RMSF}=1.510 \mathrm{e}-05 \backslash \mathrm{Dipole}=-0.3$ $278019,-0.0811157,0.775613 \backslash \mathrm{PG}=\mathrm{C} 01 \quad[\mathrm{X}(\mathrm{C} 6 \mathrm{H} 13 \mathrm{Cl} 1)] \backslash \backslash @$

Product 5-Cl) $\mathrm{CH} 3 \mathrm{OCH} 2-\mathrm{Cl}$

$1 \backslash 1 \backslash$ GINC-R6 18 $\backslash$ FOpt $\backslash$ RMP $2-F C \backslash 6-31 G(d) \backslash C 2 H 5 C l 101 \backslash R O O T \backslash 16-J a n-2016 \backslash 0 \backslash \backslash \#$ MP 2/6-31G(d) SCF=Tight INT(grid=ultrafine) OPT IOP $(2 / 17=4)$ maxdisk $=67108$ $8640 \backslash \backslash$ diMetk. MP $2 \backslash \backslash 0,1 \backslash \mathrm{H},-2.7139841267,0.4691484233,0.6251702975 \backslash \mathrm{C},-2.8$ $985246874,1.4537616965,1.0724560658 \backslash \mathrm{H},-2.7510820209,1.4016249119,2.150$ $1409521 \backslash \mathrm{H},-2.2121497665,2.1864841982,0.6387674964 \backslash \mathrm{O},-4.2575521192,1.84$ $93763381,0.8791422888 \backslash \mathrm{C},-4.6488917534,1.8372490196,-0.4440357966 \backslash \mathrm{Cl},-3$ $.9149056035,3.1739587615,-1.4230467621 \backslash \mathrm{H},-4.3682267401,0.9104035734,-0$ $.9550434172 \backslash \mathrm{H},-5.7252371822,1.9931480776,-0.4648651247 \backslash \backslash$ Version=ES64LG09RevD.01 \State $=1-\mathrm{A} \backslash \mathrm{HF}=-612.9699401 \backslash \mathrm{MP} 2=-613.5387423 \backslash \mathrm{RMSD}=6.059 \mathrm{e}-09 \backslash \mathrm{R}$ $\mathrm{MSF}=2.055 \mathrm{e}-05 \backslash \mathrm{Dipole}=0.1608576,-0.7901168,0.1974268 \backslash \mathrm{PG}=\mathrm{C} 01 \quad[\mathrm{X}(\mathrm{C} 2 \mathrm{H} 5 \mathrm{C} 11 \mathrm{O}$ $1)] \backslash \backslash @$

Product $6-\mathrm{Cl}) \mathrm{CH} 3 \mathrm{CH} 2 \mathrm{OCH} 2-\mathrm{Cl}$

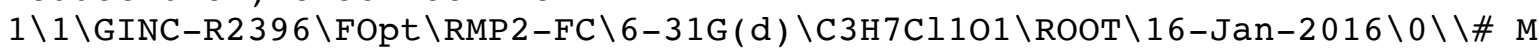
P2/6-31G(d) SCF=Tight INT(grid=ultrafine) OPT IOP(2/17=4) maxdisk=6710 $88640 \backslash \backslash d i M e t k . M P 2 \backslash \backslash 0,1 \backslash \mathrm{H},-2.6384077569,2.3580326127,-0.1669210507 \backslash \mathrm{C},-2$ $.8395253248,2.0211221235,0.8562279983 \backslash \mathrm{H},-2.2265158489,1.1290630987,1.0$ $502472903 \backslash \mathrm{C},-2.5564446083,3.114296848,1.8616316997 \backslash 0,-4.227080673,1.67$ $69814191,0.9835441625 \backslash \mathrm{C},-4.6090246871,0.6060852371,0.2015297579 \backslash \mathrm{H},-3.9$ $159611422,-0.2378666097,0.279205821 \backslash \mathrm{H},-3.1830420525,3.9844700755,1.656$ $3670653 \backslash \mathrm{H},-2.7662621213,2.7638823958,2.874268462 \backslash \mathrm{H},-1.5072662652,3.417$ 
$0107484,1.8043006254 \backslash \mathrm{Cl},-4.6974260999,1.0015771357,-1.5655955009 \backslash \mathrm{H},-5$. $6150264199,0.3240449153,0.5050786692 \backslash \backslash$ Version=ES64L-G09RevD.01\State=1 $-\mathrm{A} \backslash \mathrm{HF}=-652.0100428 \backslash \mathrm{MP} 2=-652.7099825 \backslash \mathrm{RMSD}=3.741 \mathrm{e}-09 \backslash \mathrm{RMSF}=1.074 \mathrm{e}-05 \backslash \mathrm{Dipo}$ $l e=0.5403295,-0.2477515,0.6046765 \backslash P G=C 01[X(C 3 H 7 C 1101)] \backslash \backslash @$

Product 7-Cl) $\mathrm{CH} 3 \mathrm{OC}(\mathrm{CH} 3) 2-\mathrm{Cl}$

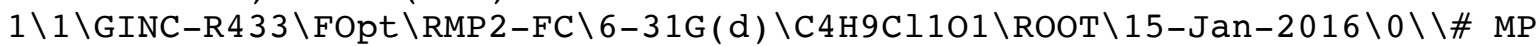
2/6-31G(d) SCF=Tight INT(grid=ultrafine) OPT IOP(2/17=4) maxdisk=12079 $59552 \backslash \backslash \mathrm{diMetk} . \mathrm{MP} 2 \backslash \backslash 0,1 \backslash \mathrm{H},-2.04294423,2.2158359904,0.8708746887 \backslash \mathrm{C},-3.04$ $53450092,1.8580951058,0.6371728597 \backslash \mathrm{H},-3.5181198488,2.5471816096,-0.069$ $426039 \backslash \mathrm{H},-2.9844546444,0.857862389,0.1986745332 \backslash \mathrm{O},-3.7353121004,1.8271$ $118784,1.8881815465 \backslash \mathrm{C},-4.9850107733,1.2078495664,1.9014891399 \backslash \mathrm{C},-5.516$ $0365502,1.3960766171,3.3070073986 \backslash \mathrm{Cl},-4.7907489493,-0.603939625,1.6244$ $796704 \backslash \mathrm{H},-6.4668782409,0.8757501597,3.4342596469 \backslash \mathrm{H},-4.7914750742,1.009$ $9627025,4.0253121192 \backslash \mathrm{H},-5.6585647268,2.4655517581,3.4852871135 \backslash \mathrm{C},-5.93$ $5538319,1.7215329892,0.8337694391 \backslash \mathrm{H},-5.5851290821,1.4826366465,-0.1712$ $494339 \backslash \mathrm{H},-6.0195411455,2.8079127954,0.9378872635 \backslash \mathrm{H},-6.9217753058,1.272$ $4424168,0.9653860536 \backslash \backslash$ Version=ES64L-G09RevD.01 \State=1-A \HF=-691.04765 $8 \backslash M P 2=-691.8864454 \backslash \mathrm{RMSD}=4.302 e-09 \backslash \mathrm{RMSF}=7.584 \mathrm{e}-06 \backslash \mathrm{Dipole}=-0.2060821,0.7$ $512805,-0.3758398 \backslash \mathrm{PG}=\mathrm{C} 01 \quad[\mathrm{X}(\mathrm{C} 4 \mathrm{H} 9 \mathrm{Cl} 101)] \backslash \backslash @$

Product 8-Cl) $\mathrm{CH} 3 \mathrm{CH} 2 \mathrm{OC}(\mathrm{CH} 3) 2-\mathrm{Cl}$

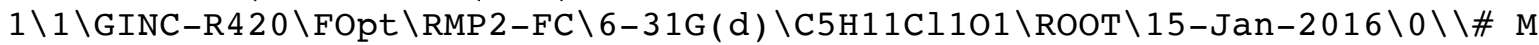
P2/6-31G(d) SCF=Tight INT(grid=ultrafine) OPT IOP(2/17=4) maxdisk=1476 $395008 \backslash \backslash$ diMetk.MP2 $\backslash \backslash 0,1 \backslash \mathrm{H},-2.5371995916,0.9003797856,0.8107412418 \backslash \mathrm{C},-3$ $.0391202794,1.7105757896,1.3534958333 \backslash \mathrm{H},-3.0931627581,1.4405405797,2.4$ $139339694 \backslash \mathrm{C},-2.3093806645,3.0222599175,1.1611590116 \backslash 0,-4.3647256102,1$. $9026470424,0.8363108006 \backslash \mathrm{C},-5.321318077,0.9412239134,1.1602721438 \backslash \mathrm{C},-4$. $882798954,-0.4830986932,0.8671914743 \backslash \mathrm{C},-6.5735691384,1.3422957514,0.40$ $9174216 \backslash \mathrm{H},-2.2966689651,3.2967119998,0.1040744347 \backslash \mathrm{H},-2.8087646178,3.81$ $51427849,1.7212911913 \backslash \mathrm{H},-1.2772577903,2.9368788082,1.5130558758 \backslash \mathrm{H},-7.4$ $05374832,0.6828905167,0.6631771189 \backslash \mathrm{H},-6.8357358406,2.371053111,0.66028$ $27905 \backslash \mathrm{H},-6.3717772849,1.2783584866,-0.6637483674 \backslash \mathrm{H},-5.7072882796,-1.17$ $40088956,1.0538704479 \backslash \mathrm{H},-4.5931847401,-0.5465333318,-0.1864990359 \backslash \mathrm{H},-4$ $.0389112894,-0.7830567699,1.4900058986 \backslash \mathrm{Cl},-5.7106682868,1.0270362038,2$ $.9611769548 \backslash \backslash$ Version=ES64L-G09RevD.01\State $=1-\mathrm{A} \backslash \mathrm{HF}=-730.0875126 \backslash \mathrm{MP} 2=-7$ $31.0578475 \backslash \mathrm{RMSD}=7.589 \mathrm{e}-09 \backslash \mathrm{RMSF}=1.011 \mathrm{e}-05 \backslash \mathrm{Dipole}=0.4555512,-0.369756,-0$ $.6222486 \backslash \mathrm{PG}=\mathrm{C} 01 \quad[\mathrm{X}(\mathrm{C} 5 \mathrm{H} 11 \mathrm{C} 1101)] \backslash \backslash @$

Product 9-Cl) HOCH2 $\mathrm{CH} 2-\mathrm{Cl}$

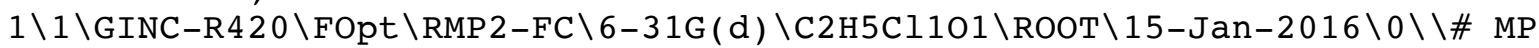
2/6-31G(d) SCF=Tight INT(grid=ultrafine) OPT IOP(2/17=4) maxdisk=67108

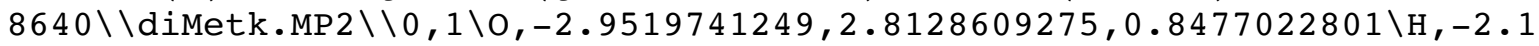
$53160341,2.4736616562,1.2884693367 \backslash \mathrm{C},-4.0273741316,1.9806280538,1.2478$ $631807 \backslash \mathrm{C},-3.863733009,0.5519662001,0.7646702054 \backslash \mathrm{H},-4.9230860512,2.4024$ $287123,0.7826272332 \backslash \mathrm{H},-4.1690026606,1.9907014061,2.3369738189 \backslash \mathrm{H},-3.695$ $112775,0.5347964512,-0.3123572283 \backslash \mathrm{Cl},-2.4371977061,-0.2085214988,1.536$ $819166 \backslash \mathrm{H},-4.7347402006,-0.0564469085,1.0155610072 \backslash \backslash$ Version=ES64L-G09Re $\mathrm{VD} .01 \backslash \mathrm{State}=1-\mathrm{A} \backslash \mathrm{HF}=-612.9774706 \backslash \mathrm{MP} 2=-613.5478017 \backslash \mathrm{RMSD}=6.282 \mathrm{e}-09 \backslash \mathrm{RMSF}=3$ $.131 e-05 \backslash \mathrm{Dipole}=-0.5594689,-0.169583,0.0795133 \backslash \mathrm{PG}=\mathrm{C} 01 \quad[\mathrm{X}(\mathrm{C} 2 \mathrm{H} 5 \mathrm{C} 1101)] \backslash \backslash$ a

Product $10-\mathrm{Cl})$ HOCH $2 \mathrm{C}(\mathrm{CH} 3) 2-\mathrm{Cl}$

$1 \backslash 1 \backslash G I N C-R 2738 \backslash F O p t \backslash R M P 2-F C \backslash 6-31 G(d) \backslash C 4 H 9 C 1101 \backslash R O O T \backslash 15-J a n-2016 \backslash 0 \backslash \backslash \# M$ P2/6-31G(d) SCF=Tight INT(grid=ultrafine) OPT IOP(2/17=4) maxdisk=1207 $959552 \backslash \backslash \mathrm{diMetk} . \mathrm{MP} 2 \backslash \backslash 0,1 \backslash \mathrm{O},-2.840906341,2.0853440264,0.7537664576 \backslash \mathrm{H},-2$. $5429234648,1.3612022594,1.3333469309 \backslash \mathrm{C},-4.2503575159,2.1507992561,0.88$ $38330404 \backslash \mathrm{C},-4.9716802869,0.8988960553,0.3846983586 \backslash \mathrm{H},-4.5688985992,2.9$ $959480155,0.2646985561 \backslash \mathrm{H},-4.5534311214,2.3512117275,1.9214031775 \backslash \mathrm{Cl},-4$ $.4277154387,-0.4721985834,1.4520708633 \backslash \mathrm{C},-4.5787955164,0.5635597757,-1$ 
$.043489274 \backslash \mathrm{H},-5.0454665173,-0.3708986409,-1.3639967074 \backslash \mathrm{H},-4.9169780774$ $, 1.36878987,-1.7046145924 \backslash \mathrm{H},-3.495370902,0.4766783497,-1.1317993283 \backslash \mathrm{C}$, $-6.4750128092,1.0489620577,0.5490811034 \backslash \mathrm{H},-6.9887644586,0.1359023743,0$ $.2390886349 \backslash \mathrm{H},-6.7389145248,1.2597718116,1.5887293473 \backslash \mathrm{H},-6.8273074264$, $1.8751436452,-0.0778105679 \backslash \backslash$ Version=ES64L-G09RevD.01 State $=1-\mathrm{A} \backslash \mathrm{HF}=-691$ $.0525668 \backslash M P 2=-691.8929409 \backslash R M S D=2.805 e-09 \backslash R M S F=1.138 e-05 \backslash D i p o l e=-0.6258$ $622,0.2018995,-0.1500518 \backslash \mathrm{PG}=\mathrm{C} 01 \quad[\mathrm{X}(\mathrm{C} 4 \mathrm{H} 9 \mathrm{Cl} 101)] \backslash \backslash @$

Product $11-\mathrm{Cl}) \mathrm{CH} 3 \mathrm{OCH} 2 \mathrm{CH} 2-\mathrm{Cl}$

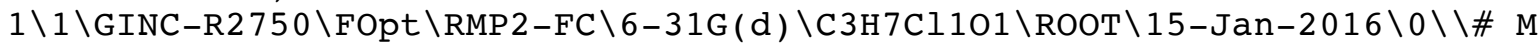
P2/6-31G(d) SCF=Tight INT(grid=ultrafine) OPT IOP(2/17=4) maxdisk=1207 $959552 \backslash \backslash$ diMetk.MP2 $\backslash \backslash 0,1 \backslash 0,-3.166941988,1.9838967969,2.1906760767 \backslash \mathrm{C},-2$. $375278797,3.0639002782,1.7176464009 \backslash \mathrm{C},-4.4023935633,1.9064848161,1.502$ $0000951 \backslash \mathrm{C},-5.1375059215,0.7246633853,2.0997648178 \backslash \mathrm{H},-4.9890933303,2.82$ $78285275,1.6335574015 \backslash \mathrm{H},-4.2473014956,1.754362608,0.423481043 \backslash \mathrm{H},-5.305$ $4968402,0.8816575815,3.1650293542 \backslash \mathrm{H},-1.4511421505,3.0508167638,2.29585$ $40659 \backslash \mathrm{H},-2.1398911642,2.9483788172,0.6512627558 \backslash \mathrm{H},-2.8842218902,4.0257$ $233437,1.8652952708 \backslash \mathrm{H},-4.5643226094,-0.1909792655,1.9558360876 \backslash \mathrm{Cl},-6.7$ $238372496,0.5195313474,1.3093876304 \backslash \backslash$ Version=ES64L-G09RevD. $01 \backslash$ State=1$\mathrm{A} \backslash \mathrm{HF}=-652.0045692 \backslash \mathrm{MP} 2=-652.7022962 \backslash \mathrm{RMSD}=4.835 e-09 \backslash \mathrm{RMSF}=1.045 e-05 \backslash \mathrm{Dipol}$ $\mathrm{e}=0.6405887,0.5054553,-0.0558412 \backslash \mathrm{PG}=\mathrm{C} 01 \quad[\mathrm{X}(\mathrm{C} 3 \mathrm{H} 7 \mathrm{Cl1O1})] \backslash \backslash \mathrm{Q}$

Product $12-\mathrm{Cl}) \mathrm{CH} 3 \mathrm{OCH} 2 \mathrm{C}(\mathrm{CH} 3) 2-\mathrm{Cl}$

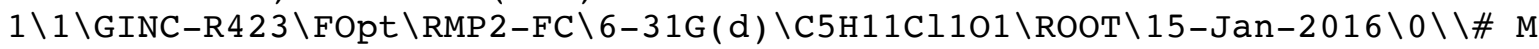
P2/6-31G(d) SCF=Tight INT(grid=ultrafine) OPT IOP(2/17=4) maxdisk=1476

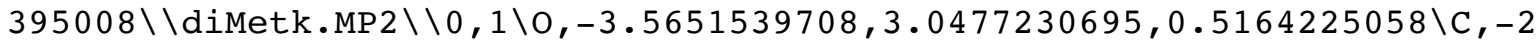
$.5916442205,2.4621494029,1.3687944469 \backslash \mathrm{C},-4.8389519592,2.4525396803,0.7$ $026681126 \backslash \mathrm{C},-5.8082374767,3.1441058847,-0.2489641205 \backslash \mathrm{H},-4.8035062649,1$ $.3743010925,0.4817705078 \backslash \mathrm{H},-5.1816754491,2.5779163639,1.7416451454 \backslash \mathrm{C},-$ $5.4085430072,2.9216622744,-1.6981646099 \backslash \mathrm{C},-5.9427102158,4.6213532288,0$ $.0810956634 \backslash \mathrm{H},-6.6692078408,5.0974687622,-0.5821358803 \backslash \mathrm{H},-6.2715933292$ $, 4.7589794303,1.114496836 \backslash \mathrm{H},-4.9695573718,5.1019475819,-0.0457257029 \backslash \mathrm{H}$ $,-1.6495125185,2.9717071575,1.1646187461 \backslash \mathrm{H},-2.856782973,2.5948781284,2$ $.4261633092 \backslash \mathrm{H},-2.4779028233,1.3890690692,1.1650628664 \backslash \mathrm{Cl},-7.4106974223$ , $2.3466949337,0.0316775533 \backslash \mathrm{H},-6.1321814439,3.3884987967,-2.3710465576 \backslash$ $\mathrm{H},-4.4233800565,3.3640059539,-1.8650573128 \backslash \mathrm{H},-5.3585806565,1.853796189$ ,$-1.926589509 \backslash \backslash$ Version=ES6 4L-G09RevD.01 $\mathbf{s}$ tate $=1-\mathrm{A} \backslash \mathrm{HF}=-730.0793318 \backslash \mathrm{MP} 2=$ $-731.0481894 \backslash \mathrm{RMSD}=3.280 \mathrm{e}-09 \backslash \mathrm{RMSF}=6.250 \mathrm{e}-06 \backslash \mathrm{Dipole}=0.8013662,0.0156072$, $0.2259913 \backslash \mathrm{PG}=\mathrm{C} 01 \quad[\mathrm{X}(\mathrm{C} 5 \mathrm{H} 11 \mathrm{C} 1101)] \backslash \backslash @$

Product $13-\mathrm{Cl}$ ) HOOCH2-Cl

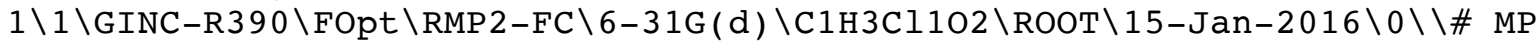
2/6-31G(d) SCF=Tight INT(grid=ultrafine) OPT IOP(2/17=4) maxdisk=12079 $59552 \backslash \backslash$ diMetk.MP2 \\0,1\O,-3.1624695345,2.1088581994, $1.8580249657 \backslash \mathrm{H},-3$. $1776292754,1.5383274041,2.6541592944 \backslash 0,-3.939330296,1.2704268465,0.945$ $8012714 \backslash \mathrm{C},-5.2351193356,1.7805652786,0.9542433469 \backslash \mathrm{H},-5.2356167676,2.86$ $60066578,0.8651902739 \backslash \mathrm{Cl},-6.1291631279,1.3685411841,2.4566949149 \backslash \mathrm{H},-5$. $7348966629,1.2896184295,0.1195969328 \backslash \backslash$ Version=ES64L-G09RevD.01\State=1 $-\mathrm{A} \backslash \mathrm{HF}=-648.6971199 \backslash \mathrm{MP} 2=-649.3243668 \backslash \mathrm{RMSD}=8.202 \mathrm{e}-09 \backslash \mathrm{RMSF}=4.150 \mathrm{e}-05 \backslash \mathrm{Dipo}$ $l e=-0.1250904,0.0375308,-0.0704017 \backslash \mathrm{PG}=\mathrm{C} 01 \quad[\mathrm{X}(\mathrm{C} 1 \mathrm{H} 3 \mathrm{Cl1O2})] \backslash \backslash @$

Product $14-\mathrm{Cl})$ HOOC ( $\mathrm{CH} 3) 2-\mathrm{Cl}$

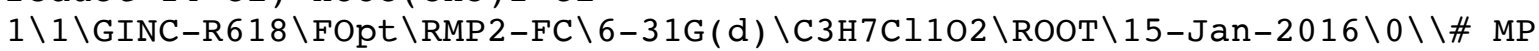
$2 / 6-31 \mathrm{G}(\mathrm{d}) \mathrm{SCF}=\mathrm{Tight}$ INT(grid=ultrafine) OPT IOP $(2 / 17=4$ ) maxdisk=20132 $65920 \backslash \backslash$ diMetk. MP $2 \backslash \backslash 0,1 \backslash 0,-3.2660924802,2.4937765651,1.6593102074 \backslash \mathrm{H},-3$. $0556311762,1.738834644,2.2482983814 \backslash 0,-3.9454672576,1.7699250646,0.586$ $7556559 \backslash \mathrm{C},-5.3268877199,1.7237289718,0.862203748 \backslash \mathrm{C},-5.9086450897,1.036$ $540842,-0.3573797554 \backslash \mathrm{C},-5.9207962806,3.0856264239,1.147538087 \backslash \mathrm{H},-6.998$ $8559986,2.9988261452,1.2966847782 \backslash \mathrm{H},-5.4659071395,3.5256489311,2.03332$ $81444 \backslash \mathrm{H},-5.7239396162,3.7311612253,0.2869858744 \backslash \mathrm{H},-6.9802943278,0.8835$ 
$107726,-0.2189506288 \backslash \mathrm{H},-5.7431136813,1.669314614,-1.2330214852 \backslash \mathrm{H},-5.42$ $50404479,0.0708503533,-0.5129755376 \backslash \mathrm{Cl},-5.6052277845,0.6542104469,2.31$ 79185302 \Version=ES64L-G09RevD. 01 State $=1-A \backslash H F=-726.7774674 \backslash M P 2=-727$. $674865 \backslash \mathrm{RMSD}=8.004 \mathrm{e}-09 \backslash \mathrm{RMSF}=3.405 \mathrm{e}-05 \backslash \mathrm{Dipole}=-0.3508197,0.0459333,-0.19$ $48449 \backslash \mathrm{PG}=\mathrm{C} 01 \quad[\mathrm{X}(\mathrm{C} 3 \mathrm{H} 7 \mathrm{Cl} 102)] \backslash \backslash \mathrm{Q}$

Product $15-\mathrm{Cl}$ ) $\mathrm{CH} 300 \mathrm{CH} 2-\mathrm{Cl}$

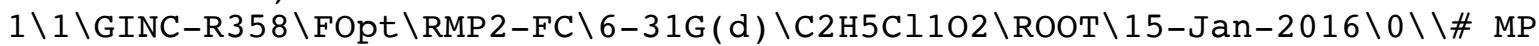
2/6-31G(d) SCF=Tight INT(grid=ultrafine) OPT IOP(2/17=4) maxdisk=14763 $95008 \backslash \backslash d i M e t k . M P 2 \backslash \backslash 0,1 \backslash 0,-2.9708357601,1.829568245,1.2149488039 \backslash \mathrm{C},-2.4$ $679289569,3.0799145164,1.6838015187 \backslash 0,-4.3080696762,1.7132061226,1.824$ $5238003 \backslash \mathrm{C},-5.1658306983,1.4318955104,0.7673893208 \backslash \mathrm{Cl},-5.5367443992,2.8$ $633430258,-0.2511863018 \backslash \mathrm{H},-1.4632223192,3.1296142874,1.258187274 \backslash \mathrm{H},-3$. $0796612099,3.9088121613,1.3215819774 \backslash \mathrm{H},-2.4140770841,3.0900031964,2.77$ $55408436 \backslash \mathrm{H},-4.7475012766,0.6699798918,0.1103120374 \backslash \mathrm{H},-6.0934946195,1.1$ $075100427,1.2388347258 \backslash \backslash$ Version=ES64L-G09RevD. $01 \backslash$ State $=1-A \backslash H F=-687.727$ $6551 \backslash M P 2=-688.4846492 \backslash \mathrm{RMSD}=3.941 \mathrm{e}-09 \backslash \mathrm{RMSF}=3.473 e-05 \backslash \mathrm{Dipole}=0.1830814,-$ $0.0911914,0.2273999 \backslash \mathrm{PG}=\mathrm{C} 01 \quad[\mathrm{X}(\mathrm{C} 2 \mathrm{H} 5 \mathrm{C} 1102)] \backslash \backslash @$

Product $16-\mathrm{Cl}) \mathrm{CH} 30 \mathrm{OC}(\mathrm{CH} 3) 2-\mathrm{Cl}$

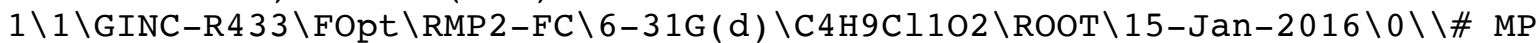
2/6-31G(d) SCF=Tight INT(grid=ultrafine) OPT IOP(2/17=4) maxdisk=22817

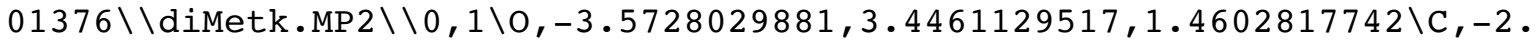
$3746853766,2.9985885631,2.0929330179 \backslash 0,-3.956410903,2.3239978201,0.584$ $024022 \backslash \mathrm{C},-5.288488854,1.9802406053,0.8745077507 \backslash \mathrm{C},-5.6108109285,0.9167$ $8765,-0.1588596191 \backslash \mathrm{C},-6.2232698522,3.1712993803,0.838937627 \backslash \mathrm{H},-2.07387$ $57042,3.8505065952,2.7074065688 \backslash \mathrm{H},-2.5649579617,2.1234889345,2.7173573$ $201 \backslash \mathrm{H},-1.6027875983,2.7809595002,1.3497672512 \backslash \mathrm{H},-7.2525258208,2.841225$ $3443,0.9942663031 \backslash \mathrm{H},-5.9549464222,3.8912610135,1.6105075106 \backslash \mathrm{H},-6.13785$ $53278,3.6471544718,-0.1420521361 \backslash \mathrm{H},-6.6145128318,0.5256049546,0.016614$ $3872 \backslash \mathrm{H},-5.563905829,1.3619792742,-1.1559160968 \backslash \mathrm{H},-4.8914824476,0.09869$ $44552,-0.0936634529 \backslash \mathrm{Cl},-5.3908461544,1.222649486,2.529931772 \backslash \backslash$ Version= ES64L-G09RevD.01 \State $=1-A \backslash H F=-765.8069272 \backslash M P 2=-766.834721 \backslash \mathrm{RMSD}=7.660 \mathrm{e}$ $-09 \backslash \mathrm{RMSF}=3.450 \mathrm{e}-06 \backslash \mathrm{Dipole}=0.0770184,0.0785199,-0.3306543 \backslash \mathrm{PG}=\mathrm{C} 01 \quad[\mathrm{X}(\mathrm{C} 4 \mathrm{H}$ $9 \mathrm{Cl} 102$ ) $] \backslash \backslash$ Q

Product $17-\mathrm{Cl}) \mathrm{HSCH} 2 \mathrm{CH} 2-\mathrm{Cl}$

$1 \backslash 1 \backslash G I N C-R 512 \backslash F O p t \backslash R M P 2-F C \backslash 6-31 G(d) \backslash C 2 H 5 C 11 S 1 \backslash R O O T \backslash 15-J a n-2016 \backslash 0 \backslash \backslash \#$ MP 2/6-31G(d) SCF=Tight INT(grid=ultrafine) OPT IOP(2/17=4) maxdisk=67108 $8640 \backslash \backslash$ diMetk.MP2 $\backslash \backslash 0,1 \backslash \mathrm{S},-2.7141308261,2.5044777556,0.9718544439 \backslash \mathrm{H},-2.2$ $509375965,1.257074626,1.1440351809 \backslash \mathrm{C},-4.4650079273,2.1136108136,1.2705$ $477386 \backslash \mathrm{C},-5.0508958657,1.2733234837,0.1513895022 \backslash \mathrm{H},-4.9820766949,3.074$ $1948991,1.3261582244 \backslash \mathrm{H},-4.5869851557,1.6153572332,2.2343948264 \backslash \mathrm{H},-4.55$ $05397957,0.3064806258,0.0775971523 \backslash \mathrm{H},-4.9688319396,1.7884131978,-0.805$ $6979397 \backslash \mathrm{Cl},-6.7853561985,0.9514083653,0.4528758709 \backslash \backslash$ Version $=\mathrm{ES} 64 \mathrm{~L}-\mathrm{G} 09 \mathrm{R}$ evD.01 \State $=1-A \backslash H F=-935.6370649 \backslash M P 2=-936.1491952 \backslash \mathrm{RMSD}=7.932 \mathrm{e}-09 \backslash \mathrm{RMSF}=$ $7.005 e-06 \backslash \mathrm{Dipole}=0.3817842,-0.2814172,0.0533464 \backslash \mathrm{PG}=\mathrm{C} 01 \quad[\mathrm{X}(\mathrm{C} 2 \mathrm{H} 5 \mathrm{C} 11 \mathrm{~S} 1)] \backslash$ $\backslash$ a

Product $18-\mathrm{Cl}) \mathrm{HSCH} 2 \mathrm{C}(\mathrm{CH} 3) 2-\mathrm{Cl}$

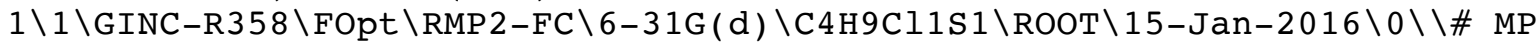
2/6-31G(d) SCF=Tight INT(grid=ultrafine) OPT IOP(2/17=4) maxdisk=22817 $01376 \backslash \backslash$ diMetk.MP $2 \backslash \backslash 0,1 \backslash \mathrm{S},-2.8517739172,2.195880988,0.7308963023 \backslash \mathrm{H},-2.6$ $564327069,1.2573163892,1.6676541514 \backslash \mathrm{C},-4.664852008,2.1980719897,0.8297$ $624709 \backslash \mathrm{C},-5.3989162354,0.9449736096,0.3449841737 \backslash \mathrm{H},-4.9832089707,3.032$ $6194347,0.1950743745 \backslash \mathrm{H},-4.9764037801,2.4261644293,1.8527047583 \backslash \mathrm{Cl},-4.9$ $530168463,-0.4316456159,1.4391376746 \backslash \mathrm{C},-5.0323987256,0.5567075358,-1.0$ $776380322 \backslash \mathrm{H},-5.5876275611,-0.3344581956,-1.3800698129 \backslash \mathrm{H},-5.294892173,1$ $.3780808743,-1.7534745676 \backslash \mathrm{H},-3.963837889,0.3588867318,-1.169964855 \backslash \mathrm{C},-$ $6.9001858429,1.1657620084,0.4865688062 \backslash \mathrm{H},-7.4475245555,0.2655665674,0$. 
$1967628192 \backslash \mathrm{H},-7.1654334925,1.4164210199,1.5168692227 \backslash \mathrm{H},-7.2110212959,1$ $.9877682333,-0.1670584862 \backslash \backslash$ Version=ES64L-G09RevD. $01 \backslash$ State $=1-A \backslash H F=-1013$ $.7083033 \backslash M P 2=-1014.492393 \backslash \mathrm{RMSD}=3.948 \mathrm{e}-09 \backslash \mathrm{RMSF}=3.496 \mathrm{e}-06 \backslash \mathrm{Dipole}=-0.7213$ $141,0.3063683,-0.2484703 \backslash P G=C 01 \quad[X(C 4 H 9 C l 1 S 1)] \backslash \backslash @$

Product $19-\mathrm{Cl}) \mathrm{CH} 3 \mathrm{SCH} 2 \mathrm{CH} 2-\mathrm{Cl}$

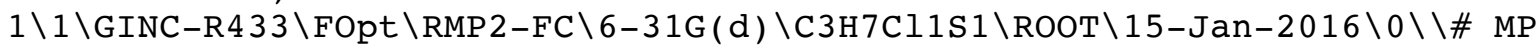
2/6-31G(d) SCF=Tight INT(grid=ultrafine) OPT IOP(2/17=4) maxdisk=17448 $30464 \backslash \backslash$ diMetk. MP $2 \backslash \backslash 0,1 \backslash S,-2.7779398369,2.8091717439,0.2973580012 \backslash \mathrm{C},-2$. $5485549967,2.9711843307,2.0854575849 \backslash \mathrm{C},-4.570290475,2.5474763609,0.236$ $3686135 \backslash \mathrm{C},-4.9569565157,1.1383395989,0.6476850803 \backslash \mathrm{H},-4.8767133117,2.72$ $83928622,-0.7973366964 \backslash \mathrm{H},-5.0750189114,3.2867284233,0.8656856852 \backslash \mathrm{H},-4$. $6536472041,0.9190283677,1.6716927249 \backslash \mathrm{H},-4.5072065274,0.403334851,-0.01$ $99106319 \backslash \mathrm{H},-1.4994210172,3.2246846273,2.2500063121 \backslash \mathrm{H},-3.1669549055,3.7$ $781805776,2.4855674566 \backslash \mathrm{H},-2.763924086,2.0437547453,2.6190634164 \backslash \mathrm{Cl},-6$. $7337952124,0.925884511,0.5707994533 \backslash \backslash$ Version=ES64L-G09RevD . 01\State=1$\mathrm{A} \backslash \mathrm{HF}=-974.6711136 \backslash \mathrm{MP} 2=-975.3172853 \backslash \mathrm{RMSD}=3.448 e-09 \backslash \mathrm{RMSF}=4.040 e-06 \backslash \mathrm{Dipol}$ $\mathrm{e}=0.433859,0.164313,0.5481281 \backslash \mathrm{PG}=\mathrm{C} 01 \quad[\mathrm{X}(\mathrm{C} 3 \mathrm{H} 7 \mathrm{Cl} 1 \mathrm{~S} 1)] \backslash \backslash \mathrm{Q}$

Product 20-Cl) $\mathrm{CH} 3 \mathrm{SCH} 2 \mathrm{C}(\mathrm{CH} 3) 2-\mathrm{Cl}$

$1 \backslash 1 \backslash G I N C-R 2738 \backslash F O p t \backslash R M P 2-F C \backslash 6-31 G(d) \backslash C 5 H 11 C 11 S 1 \backslash R O O T \backslash 15-J a n-2016 \backslash 0 \backslash \backslash \#$ MP2 /6-31G(d) SCF=Tight INT(grid=ultrafine) OPT IOP(2/17=4) maxdisk=442 $9185024 \backslash \backslash d i M e t k . M P 2 \backslash \backslash 0,1 \backslash S,-2.8987480516,2.2642493337,0.4173889411 \backslash \mathrm{C},-$ $2.4411121342,3.1165767171,1.947597535 \backslash \mathrm{C},-4.7125309137,2.3747590367,0.4$ $977793242 \backslash \mathrm{C},-5.4139043226,1.133006019,1.0561939574 \backslash \mathrm{H},-5.06739918,2.548$ $415026,-0.5224689409 \backslash \mathrm{H},-4.98311124,3.253823011,1.0914849154 \backslash \mathrm{C},-5.02979$ $46217,0.8342050998,2.4962790387 \backslash \mathrm{C},-5.2129627559,-0.0808348059,0.162598$ $5924 \backslash \mathrm{H},-1.3523821899,3.2035174685,1.9461415698 \backslash \mathrm{H},-2.8678079241,4.12176$ $58618,1.9748306674 \backslash \mathrm{H},-2.7438386453,2.5638175848,2.8378156243 \backslash \mathrm{H},-5.7797$ $279277,-0.9332494679,0.5450751077 \backslash \mathrm{H},-5.5467213308,0.1284873122,-0.8572$ $092582 \backslash \mathrm{H},-4.1513470627,-0.3431104543,0.1409907538 \backslash \mathrm{Cl},-7.1865531944,1.5$ $419405027,1.0368340991 \backslash \mathrm{H},-5.6223972065,0.0004093163,2.8804792026 \backslash \mathrm{H},-3$. $9724661374,0.557256408,2.5386700882 \backslash \mathrm{H},-5.2016301616,1.7038710305,3.136$ $324782 \backslash \backslash$ Version=ES64L-G09RevD.01 \State $=1-A \backslash H F=-1052.741269 \backslash \mathrm{MP} 2=-1053.6$ $599761 \backslash \mathrm{RMSD}=5.571 \mathrm{e}-09 \backslash \mathrm{RMSF}=1.541 \mathrm{e}-05 \backslash \mathrm{Dipole}=0.5273155,0.0627037,0.5077$ $56 \backslash P G=C 01 \quad[X(C 5 H 11 C 11 S 1)] \backslash \backslash @$

Product 21-Cl) $\mathrm{CH} 3 \mathrm{SCH} 2-\mathrm{Cl}$

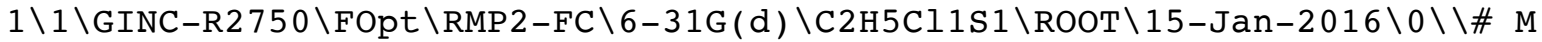
P2/6-31G(d) SCF=Tight INT(grid=ultrafine) OPT IOP(2/17=4) maxdisk=9395 $24096 \backslash \backslash$ diMetk. MP $2 \backslash \backslash 0,1 \backslash \mathrm{Cl},-2.5006923586,0.2823612678,0.1463741405 \backslash \mathrm{C},-2$ $.7751844384,1.5662262013,1.3700602563 \backslash \mathrm{H},-2.4092117271,1.1893645525,2.3$ $250887049 \backslash \mathrm{H},-2.1717495198,2.4218711014,1.0646944689 \backslash \mathrm{S},-4.4857738905,2$. $0393292701,1.6046657775 \backslash \mathrm{C},-4.7855388712,2.8372587304,0.0117103858 \backslash \mathrm{H},-4$ $.1196978696,3.690899026,-0.1345060153 \backslash \mathrm{H},-4.6640090996,2.1259725194,-0$. $8067039986 \backslash \mathrm{H},-5.8171342253,3.1936223311,0.01950628 \backslash \backslash$ Version=ES64L-G09R evD.01 $\backslash$ State $=1-\mathrm{A} \backslash \mathrm{HF}=-935.6319731 \backslash \mathrm{MP} 2=-936.1475071 \backslash \mathrm{RMSD}=4.141 \mathrm{e}-09 \backslash \mathrm{RMSF}=$ $1.878 e-05 \backslash \mathrm{Dipole}=0.0929241,0.7194906,-0.0556534 \backslash \mathrm{PG}=\mathrm{C} 01 \quad[\mathrm{X}(\mathrm{C} 2 \mathrm{H} 5 \mathrm{Cl} 1 \mathrm{~S} 1)] \backslash$ $1 \mathrm{a}$

Product 22-Cl) $\mathrm{CH} 3 \mathrm{SC}(\mathrm{CH} 3) 2-\mathrm{Cl}$

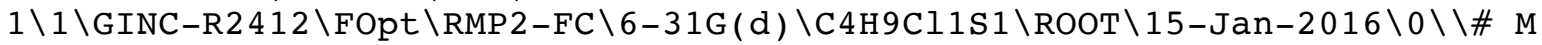
P2/6-31G(d) SCF=Tight INT(grid=ultrafine) OPT IOP(2/17=4) maxdisk=9395 $24096 \backslash \backslash$ diMetk. MP $2 \backslash \backslash 0,1 \backslash \mathrm{H},-3.4519298258,2.8389823323,-0.2641966733 \backslash \mathrm{C},-3$ $.1728152315,2.4773297203,0.7263921863 \backslash \mathrm{H},-2.2853634255,1.8450231121,0.6$ $619534543 \backslash \mathrm{H},-2.9428910759,3.3376051953,1.3585659252 \backslash \mathrm{S},-4.5205644521,1$. $5933013575,1.5421109203 \backslash \mathrm{C},-4.6065361397,0.0606659307,0.5713399232 \backslash \mathrm{C},-5$ $.7264174679,-0.7728230744,1.1808750215 \backslash \mathrm{C},-4.8399364763,0.3108112227,-0$ $.911119033 \backslash \mathrm{H},-4.9338165259,-0.644621668,-1.4348201792 \backslash \mathrm{H},-5.763653502,0$ $.8819982499,-1.0392825531 \backslash \mathrm{H},-4.0146436935,0.8644800451,-1.3620531244 \backslash \mathrm{H}$ 
$,-6.6757827733,-0.2383330216,1.0825099341 \backslash \mathrm{H},-5.8005736445,-1.727465109$ $9,0.6541839039 \backslash \mathrm{H},-5.5352297827,-0.9690933679,2.2379499133 \backslash \mathrm{Cl},-3.064789$ $9834,-0.8892099241,0.7256323809 \backslash \backslash$ Version=ES64L-G09RevD . $01 \backslash$ State $=1-A \backslash H F$ $=-1013.7036917 \backslash M P 2=-1014.4909919 \backslash \mathrm{RMSD}=2.972 \mathrm{e}-09 \backslash \mathrm{RMSF}=3.276 \mathrm{e}-06 \backslash \mathrm{Dipole}=$ $-0.3132388,0.3081572,-0.5687251 \backslash \mathrm{PG}=\mathrm{C} 01 \quad[\mathrm{X}(\mathrm{C} 4 \mathrm{H} 9 \mathrm{Cl} 1 \mathrm{~S} 1)] \backslash \backslash \mathrm{a}$

Product 23-Cl) $\mathrm{CH} 3 \mathrm{CH} 2 \mathrm{SCH} 2-\mathrm{Cl}$

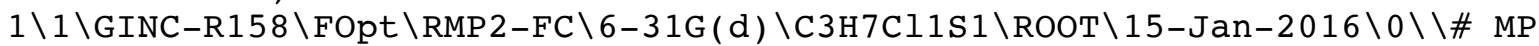
2/6-31G(d) SCF=Tight INT(grid=ultrafine) OPT IOP(2/17=4) maxdisk=93952 $4096 \backslash \backslash d i M e t k . M P 2 \backslash \backslash 0,1 \backslash H,-3.0779219607,1.3021003365,0.1710588075 \backslash \mathrm{C},-3.0$ $319705392,1.6371178099,1.2111715966 \backslash \mathrm{H},-2.9254825394,0.7438162602,1.833$ $8177871 \backslash \mathrm{C},-1.8606850287,2.5833503213,1.4380385816 \backslash \mathrm{S},-4.655136159,2.311$ $3110481,1.668175692 \backslash \mathrm{C},-4.8335434062,3.6020740882,0.4378851065 \backslash \mathrm{Cl},-4.96$ $87072792,3.0235253062,-1.2554911502 \backslash \mathrm{H},-1.9251155293,3.4692091074,0.801$ $3209036 \backslash \mathrm{H},-1.8205001374,2.9109033865,2.4794036299 \backslash \mathrm{H},-0.9217812898,2.07$ $54971465,1.1969473576 \backslash \mathrm{H},-3.9999370906,4.3026795061,0.4608524228 \backslash \mathrm{H},-5.7$ $535930406,4.1367266829,0.6748122649 \backslash \backslash$ Version=ES64L-G09RevD. $01 \backslash$ State $=1-$ $\mathrm{A} \backslash \mathrm{HF}=-974.6664707 \backslash \mathrm{MP} 2=-975.3146776 \backslash \mathrm{RMSD}=2.165 e-09 \backslash \mathrm{RMSF}=2.297 e-06 \backslash \mathrm{Dipol}$ $e=0.6305998,0.2535159,0.3397825 \backslash P G=C 01[X(C 3 H 7 C 11 S 1)] \backslash \backslash @$

Product 24-Cl) $\mathrm{CH} 3 \mathrm{CH} 2 \mathrm{SC}(\mathrm{CH} 3) 2-\mathrm{Cl}$

$1 \backslash 1 \backslash G I N C-R 158 \backslash F O p t \backslash R M P 2-F C \backslash 6-31 G(d) \backslash C 5 H 11 C 11 S 1 \backslash R O O T \backslash 15-J a n-2016 \backslash 0 \backslash \backslash \# M$ P2/6-31G(d) SCF=Tight INT(grid=ultrafine) OPT IOP(2/17=4) maxdisk=2281

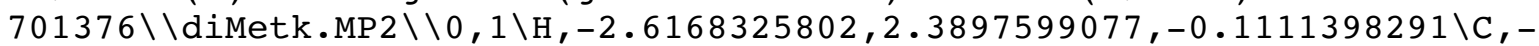
$2.9093049307,1.8699465158,0.8046992131 \backslash \mathrm{H},-2.9932184803,0.8010542752,0$. $5903759438 \backslash \mathrm{C},-1.8738768943,2.1114933543,1.8984768898 \backslash \mathrm{S},-4.5250871177,2$ $.4678214266,1.3769349156 \backslash \mathrm{C},-5.6600778628,1.7511493348,0.1534855447 \backslash \mathrm{Cl}$, $-5.6403127379,-0.065312334,0.2380290205 \backslash \mathrm{C},-7.0597862586,2.2144810451,0$ $.5368558246 \backslash \mathrm{H},-7.7904413502,1.7786575064,-0.1492685714 \backslash \mathrm{H},-7.3071765354$ $, 1.9064857362,1.5549266805 \backslash \mathrm{H},-7.1155237576,3.3048751894,0.4698932583 \backslash \mathrm{H}$ $,-1.7977451697,3.1737725546,2.14528834 \backslash \mathrm{H},-2.132476784,1.5647494209,2.8$ $08032528 \backslash \mathrm{H},-0.8913302187,1.7718926764,1.5590654449 \backslash \mathrm{C},-5.3145648012,2.1$ $428383078,-1.275761426 \backslash \mathrm{H},-4.3338310575,1.7661060874,-1.5710735013 \backslash \mathrm{H},-6$ $.0591924614,1.727995017,-1.9610742694 \backslash \mathrm{H},-5.3170030017,3.2331299785,-1$. $3611370067 \backslash \backslash$ Version=ES64L-G09RevD.01\State $=1-\mathrm{A} \backslash \mathrm{HF}=-1052.7384343 \backslash \mathrm{MP} 2=-1$ $053.6579348 \backslash \mathrm{RMSD}=9.064 \mathrm{e}-09 \backslash \mathrm{RMSF}=5.165 \mathrm{e}-06 \backslash \mathrm{Dipole}=0.2543178,0.4555827,-$ $0.4957401 \backslash \mathrm{PG}=\mathrm{C} 01 \quad[\mathrm{X}(\mathrm{C} 5 \mathrm{H} 11 \mathrm{Cl} 1 \mathrm{~S} 1)] \backslash \backslash @$

Product 25-Cl) $\mathrm{HSSCH} 2-\mathrm{Cl}$

$1 \backslash 1 \backslash G I N C-R 2412 \backslash F O p t \backslash R M P 2-F C \backslash 6-31 G(d) \backslash C 1 H 3 C 11 S 2 \backslash R O O T \backslash 15-J a n-2016 \backslash 0 \backslash \backslash \# M$ P2/6-31G(d) SCF=Tight INT(grid=ultrafine) OPT IOP(2/17=4) maxdisk=9395

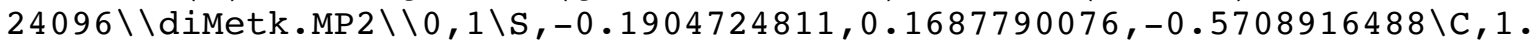
$1095105146,1.253914066,0.0440640958 \backslash \mathrm{S},-1.6133304973,1.5223871632,-1.19$ $26213758 \backslash \mathrm{H},-2.2561819945,1.7029735381,-0.0257950569 \backslash \mathrm{H}, 1.9903927342,0.6$ $252822247,0.1880535792 \backslash \mathrm{H}, 1.3256955841,2.0367237792,-0.6820278822 \backslash \mathrm{Cl}, 0$. $7445271401,2.0514112211,1.5981802887 \backslash \backslash$ Version=ES64L-G09RevD.01\State=1 $-\mathrm{A} \backslash \mathrm{HF}=-1294.1063245 \backslash \mathrm{MP} 2=-1294.6075058 \backslash \mathrm{RMSD}=7.118 \mathrm{e}-09 \backslash \mathrm{RMSF}=1.442 \mathrm{e}-05 \backslash \mathrm{Di}$ pole $=0.2500235,0.0609053,-0.0959151 \backslash P G=C 01$ [X(C1H3Cl1S2) ] \\

Product 26-Cl) $\mathrm{HSSC}(\mathrm{CH} 3) 2-\mathrm{Cl}$

$1 \backslash 1 \backslash G I N C-R 2412 \backslash F O p t \backslash R M P 2-F C \backslash 6-31 G(d) \backslash C 3 H 7 C 11 S 2 \backslash R O O T \backslash 15-J a n-2016 \backslash 0 \backslash \backslash \# M$ P2/6-31G(d) SCF=Tight INT(grid=ultrafine) OPT IOP(2/17=4) maxdisk=1476

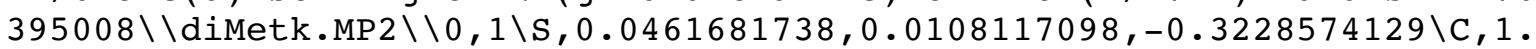
$4517100244,0.8752956773,0.4722856232 \backslash \mathrm{S},-0.8388564093,1.45173444,-1.492$ $0803399 \backslash \mathrm{H},-1.6448644705,2.0033258613,-0.5676835154 \backslash \mathrm{Cl}, 0.8496028309,2.1$ $877865338,1.5580854867 \backslash \mathrm{C}, 2.4019390491,1.4928248679,-0.5375877315 \backslash \mathrm{H}, 3.2$ $266264091,1.9823915394,-0.0125739495 \backslash \mathrm{H}, 2.8030343969,0.7064904963,-1.18$ $30568044 \backslash \mathrm{H}, 1.891440072,2.2338103405,-1.1553290555 \backslash \mathrm{C}, 2.1310301098,-0.18$ $35055721,1.3318284049 \backslash \mathrm{H}, 1.4380103038,-0.6008547058,2.0657508967 \backslash \mathrm{H}, 2.50$ $95740461,-0.9889506505,0.6955757304 \backslash \mathrm{H}, 2.9715014638,0.2697444622,1.8632$ 
806673\\Version=ES64L-G09RevD.01\State $=1-\mathrm{A} \backslash \mathrm{HF}=-1372.1795769 \backslash \mathrm{MP} 2=-1372$. $9534179 \backslash \mathrm{RMSD}=4.131 \mathrm{e}-09 \backslash \mathrm{RMSF}=1.777 \mathrm{e}-05 \backslash \mathrm{Dipole}=0.5143861,-0.0838557,0.08$ $07037 \backslash P G=C 01 \quad[X(C 3 H 7 C 11 S 2)] \backslash \backslash @$

Product 27-Cl) $\mathrm{CH} 3 \mathrm{SSCH} 2-\mathrm{Cl}$

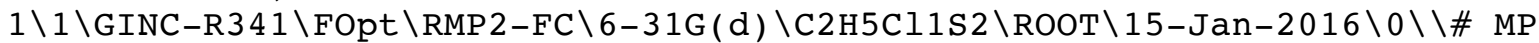
2/6-31G(d) SCF=Tight INT(grid=ultrafine) OPT IOP $(2 / 17=4)$ maxdisk=14763 $95008 \backslash \backslash$ diMetk.MP $2 \backslash \backslash 0,1 \backslash \mathrm{S},-0.0406756398,-0.055195796,-0.7597460667 \backslash \mathrm{C}, 1$. $2207320673,1.0254475221,-0.062154013 \backslash \mathrm{S},-1.6315327751,1.2171729282,-1.0$ $207580158 \backslash \mathrm{C},-1.392822568,1.8155225546,-2.7181511859 \backslash \mathrm{Cl}, 2.021683069,2.1$ $234124702,-1.2256293064 \backslash \mathrm{H}, 1.9889859489,0.3730742507,0.3572516761 \backslash \mathrm{H},-2$. $2683646274,2.4288636683,-2.9474374301 \backslash \mathrm{H},-0.491955926,2.4219185317,-2.8$ $001478933 \backslash \mathrm{H},-1.3493829624,0.9797829192,-3.4172914636 \backslash \mathrm{H}, 0.7912574135,1$. $6457129511,0.7236856988 \backslash \backslash$ Version $=E S 64 L-G 09$ RevD. $01 \backslash$ State $=1-\mathrm{A} \backslash \mathrm{HF}=-1333.1$ $428219 \backslash M P 2=-1333.7788922 \backslash R M S D=5.066 e-09 \backslash R M S F=3.008 e-05 \backslash D i p o l e=-0.00333$ $88,0.1257855,-0.0053863 \backslash \mathrm{PG}=\mathrm{C} 01 \quad[\mathrm{X}(\mathrm{C} 2 \mathrm{H} 5 \mathrm{Cl} 1 \mathrm{~S} 2)] \backslash \backslash @$

Product 28-Cl) $\mathrm{CH} 3 \mathrm{SSC}(\mathrm{CH} 3) 2-\mathrm{Cl}$

$1 \backslash 1 \backslash$ GINC-R146\FOpt \RMP2-FC \6-31G(d) \C4H9Cl1S2 2/6-31G(d) SCF=Tight INT(grid=ultrafine) OPT IOP $(2 / 17=4$ ) maxdisk=14763 $95008 \backslash \backslash d i M e t k . M P 2 \backslash \backslash 0,1 \backslash S, 0.3380118036,-0.1711357662,-0.7232507711 \backslash \mathrm{C}, 1$. $643713441,0.747661058,0.1751686624 \backslash \mathrm{S},-1.0803779681,1.230590695,-1.1992$ $511778 \backslash \mathrm{C},-2.2483769202,1.1017916169,0.184748117 \backslash \mathrm{Cl}, 1.0216136371,1.3998$ $590121,1.7450614422 \backslash \mathrm{C}, 2.1880095965,1.9130332417,-0.631588852 \backslash \mathrm{H}, 2.97668$ $56401,2.4152085049,-0.0645461515 \backslash \mathrm{H}, 2.6032772777,1.5384122587,-1.571296$ $1649 \backslash \mathrm{H}, 1.4009281375,2.6357849571,-0.8546008867 \backslash \mathrm{H},-3.086071269,1.758848$ $0899,-0.064689926 \backslash \mathrm{H},-2.6134539284,0.0790255512,0.284309864 \backslash \mathrm{H},-1.790929$ $3039,1.4310112572,1.1162382628 \backslash \mathrm{C}, 2.716707976,-0.2882267671,0.486555637$ $\backslash \mathrm{H}, 2.3121889035,-1.1083580584,1.0840357727 \backslash \mathrm{H}, 3.1241067759,-0.690025940$ $2,-0.4457352432 \backslash \mathrm{H}, 3.5244112008,0.1880592891,1.048177415 \backslash \backslash$ Version=ES64L $-G 09$ RevD.01 \State $=1-\mathrm{A} \backslash \mathrm{HF}=-1411.2155639 \backslash \mathrm{MP} 2=-1412.1246533 \backslash \mathrm{RMSD}=5.766 \mathrm{e}-0$ $9 \backslash \mathrm{RMSF}=9.850 \mathrm{e}-06 \backslash \mathrm{Dipole}=0.2447234,0.0357614,0.0921857 \backslash \mathrm{PG}=\mathrm{C} 01 \quad[\mathrm{X}(\mathrm{C} 4 \mathrm{H} 9 \mathrm{C}]$ $1 \mathrm{~S} 2) \mathrm{J} \backslash \backslash \mathrm{Q}$

\section{R-R Geometries}

\section{R-R Reactants}

\section{$\mathrm{CH} 3-\mathrm{CH} 3$}

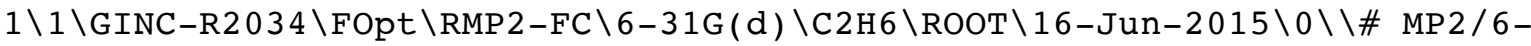
$31 \mathrm{G}(\mathrm{d})$ SCF=Tight INT(grid=ultrafine) OPT IOP(2/17=4) maxdisk=134217728 $\backslash \backslash t \mathrm{k} . \mathrm{MP} 2 \backslash \backslash 0,1 \backslash \mathrm{C},-2.6526347719,1.9727597899,-0.0007193223 \backslash \mathrm{C},-1.12818411$ $7,1.896669099,-0.0009395665 \backslash \mathrm{H},-3.0133344434,2.6519227361,0.7759564495 \backslash$ $\mathrm{H},-3.0963267376,0.9909174562,0.1828968162 \backslash \mathrm{H},-3.029434213,2.3343965762$, $-0.9608405808 \backslash \mathrm{H},-0.7513846759,1.5350323127,0.9591816919 \backslash \mathrm{H},-0.684492151$ $3,2.8785114327,-0.1845557051 \backslash \mathrm{H},-0.7674844455,1.2175061528,-0.777615338$ $4 \backslash \backslash$ Version=ES64L-G09RevD.01 \State $=1-\mathrm{A} 1 \mathrm{G} \backslash \mathrm{HF}=-79.2285325 \backslash \mathrm{MP} 2=-79.4947413$ $\backslash \mathrm{RMSD}=3.833 \mathrm{e}-09 \backslash \mathrm{RMSF}=1.785 \mathrm{e}-04 \backslash \mathrm{Dipole}=0 ., 0 ., 0 . \backslash \mathrm{PG}=\mathrm{D} 03 \mathrm{D}[\mathrm{C} 3(\mathrm{C} 1 . \mathrm{C} 1), 3 \mathrm{SGD}$ ( $\mathrm{H} 2$ ) $] \backslash \backslash @$

\section{$\mathrm{CH} 3 \mathrm{CH} 2-\mathrm{CH} 2 \mathrm{CH} 3$}

$1 \backslash 1 \backslash G I N C-R 153 \backslash F O p t \backslash R M P 2-F C \backslash 6-31 G(d) \backslash C 4 H 10 \backslash R O O T \backslash 13-J a n-2016 \backslash 0 \backslash \backslash \#$ MP2 /6$31 \mathrm{G}(d) \quad$ SCF$=$ Tight INT(grid=ultrafine) OPT IOP(2/17=4) maxdisk=134217728 $\backslash \backslash$ diMetk.MP $2 \backslash \backslash 0,1 \backslash C,-2.7404312962,1.2204036248,0.0072109586 \backslash \mathrm{C},-1.50234$ $87219,2.11341819,-0.0138010288 \backslash \mathrm{C},-0.198353818,1.3201714938,-0.00180062$ $9 \backslash \mathrm{C},-4.0440990521,2.0132678606,-0.0047828192 \backslash \mathrm{H},-2.7061686587,0.5804910$ $062,0.8979193501 \backslash \mathrm{H},-2.7110361707,0.5459285712,-0.8577993622 \backslash \mathrm{H},-1.53196$ $9554,2.788053392,0.8511209402 \backslash \mathrm{H},-1.5368365903,2.7534943348,-0.90442619$ $01 \backslash \mathrm{H},-0.1305469854,0.6956837442,0.8944461363 \backslash \mathrm{H}, 0.6732633834,1.98106906$ $25,-0.0172272666 \backslash \mathrm{H},-0.1354473926,0.6608877323,-0.8731379253 \backslash \mathrm{H},-4.91584$ 
$57217,1.3524008711,0.0106435754 \backslash \mathrm{H},-4.1072631265,2.6725638725,0.8666127$ $355 \backslash \mathrm{H},-4.112163854,2.6377655865,-0.901086851 \backslash \backslash$ Version=ES64L-G09RevD. 01 $\backslash$ State $=1-A^{\prime} \backslash H F=-157.2978862 \backslash M P 2=-157.8260365 \backslash R M S D=5.555 e-09 \backslash R M S F=5.270$ e-05\Dipole $=-0.0000138,-0.0000531,0.0000011 \backslash \mathrm{PG}=\mathrm{CS} \quad[\mathrm{SG}(\mathrm{C} 4 \mathrm{H} 2), \mathrm{X}(\mathrm{H} 8)] \backslash \backslash \mathrm{Q}$

$\mathrm{HO}-\mathrm{OH}$

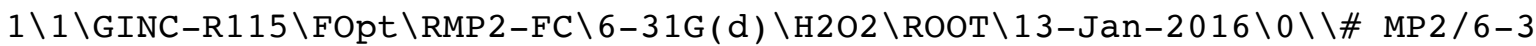
$1 \mathrm{G}(\mathrm{d}) \mathrm{SCF}=\mathrm{Tight}$ INT(grid=ultrafine) OPT IOP(2/17=4) maxdisk=134217728

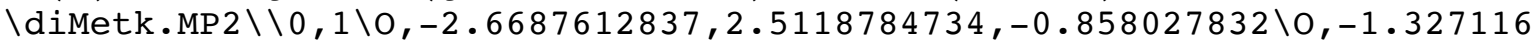
$6272,2.578153729,-1.4528095357 \backslash \mathrm{H},-2.9512217611,3.4295032297,-1.0313012$ $327 \backslash \mathrm{H},-0.805433328,2.3398635679,-0.6634933996 \backslash \backslash$ Version=ES64L-G09RevD. 0 $1 \backslash \mathrm{State}=1-\mathrm{A} \backslash \mathrm{HF}=-150.7600298 \backslash \mathrm{MP} 2=-151.1301265 \backslash \mathrm{RMSD}=5.098 \mathrm{e}-09 \backslash \mathrm{RMSF}=3.968$ e-05\Dipole $=0.178507,0.5068029,0.4594947 \backslash \mathrm{PG}=\mathrm{C} 01 \quad[\mathrm{X}(\mathrm{H} 2 \mathrm{O} 2)] \backslash \backslash @$

\section{$\mathrm{CH} 3 \mathrm{O}-\mathrm{OCH} 3$}

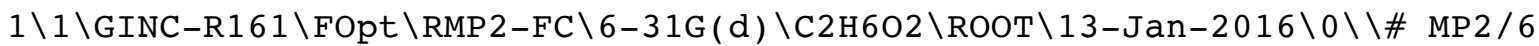
$-31 G(d)$ SCF=Tight INT(grid=ultrafine) OPT IOP(2/17=4) maxdisk=13421772

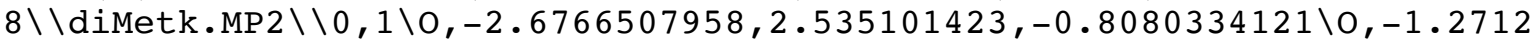
$460124,2.5592031728,-1.2479652756 \backslash \mathrm{C},-3.0608123333,3.9045397905,-0.7922$ $217347 \backslash \mathrm{C},-0.57154844,1.870937215,-0.2184484875 \backslash \mathrm{H}, 0.46683278,1.86023766$ $63,-0.5580497073 \backslash \mathrm{H},-0.651032259,2.3986378037,0.7373804534 \backslash \mathrm{H},-0.9416413$ $136,0.8475009954,-0.1112764359 \backslash \mathrm{H},-4.1123906996,3.8867496232,-0.4963408$ $869 \backslash \mathrm{H},-2.4732268854,4.4737180764,-0.0647782845 \backslash \mathrm{H},-2.9581513216,4.35064$ $9855,-1.7853595934 \backslash \backslash$ Version=ES64L-G09RevD. $01 \backslash$ State $=1-A \backslash H F=-228.8231264$ $\backslash M P 2=-229.4508818 \backslash \mathrm{RMSD}=9.124 \mathrm{e}-09 \backslash \mathrm{RMSF}=3.045 \mathrm{e}-06 \backslash \mathrm{Dipole}=0.1481573,0.319$ $8387,0.4908251 \backslash \mathrm{PG}=\mathrm{C} 02 \quad[\mathrm{X}(\mathrm{C} 2 \mathrm{H} 6 \mathrm{O} 2)] \backslash \backslash @$

\section{$\mathrm{HS}-\mathrm{SH}$}

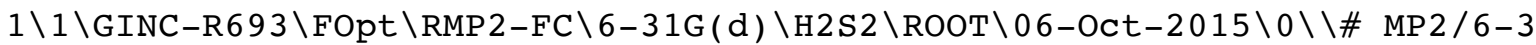
$1 \mathrm{G}(\mathrm{d})$ SCF=Tight INT(grid=ultrafine) OPT IOP(2/17=4) maxdisk=134217728 $\backslash t k . M P 2 \backslash \backslash 0,1 \backslash S,-1.7039785964,2.5850803737,-0.4263025436 \backslash \mathrm{S},-0.006632877$ $4,1.3995657886,-0.3941976401 \backslash \mathrm{H}, 0.8568286626,2.2814482441,0.1369735341 \backslash$ $\mathrm{H},-1.5638267115,3.1213461475,-1.6503130752 \backslash \backslash$ Version=ES64L-G09RevD.01\S tate $=1-\mathrm{A} \backslash \mathrm{HF}=-796.1740721 \backslash \mathrm{MP} 2=-796.4113432 \backslash \mathrm{RMSD}=3.291 \mathrm{e}-09 \backslash \mathrm{RMSF}=2.446 \mathrm{e}-0$ $5 \backslash \mathrm{Dipole}=0.3367713,0.475872,-0.2324883 \backslash \mathrm{PG}=\mathrm{C} 02$ [X(H2S2) ] \\@

\section{$\mathrm{CH} 3 \mathrm{~S}-\mathrm{SCH} 3$}

$1 \backslash 1 \backslash G I N C-R 669 \backslash F O p t \backslash R M P 2-F C \backslash 6-31 G(d) \backslash C 2 H 6 S 2 \backslash R O O T \backslash 06-O c t-2015 \backslash 0 \backslash \backslash \#$ MP $2 / 6$ $-31 \mathrm{G}(\mathrm{d}) \quad \mathrm{SCF}=\mathrm{Tight}$ INT(grid=ultrafine) OPT IOP(2/17=4) maxdisk=13421772 $8 \backslash \backslash t k . M P 2 \backslash \backslash 0,1 \backslash \mathrm{C},-2.5852868868,1.604659951,-0.0629918704 \backslash \mathrm{S},-1.44192151$ $89,2.8493717352,-0.7220973209 \backslash \mathrm{S}, 0.3131820745,1.7903071442,-0.867156218$ $1 \backslash \mathrm{H},-3.5657318585,2.0837821654,0.0034925841 \backslash \mathrm{H},-2.2863953035,1.27600657$ $39,0.9338645105 \backslash \mathrm{H},-2.6498021335,0.745764346,-0.7318822966 \backslash \mathrm{C}, 0.99451563$ $8,1.9498050871,0.8065691974 \backslash \mathrm{H}, 1.9630409189,1.4426860694,0.8002680952 \backslash \mathrm{H}$ $, 0.3515903659,1.4703969466,1.5467629519 \backslash \mathrm{H}, 1.1399681681,2.9988050514,1$. $0668016244 \backslash \backslash$ Version=ES64L-G09RevD.01\State $=1-\mathrm{A} \backslash \mathrm{HF}=-874.2471714 \backslash \mathrm{MP} 2=-87$ $4.7532122 \backslash \mathrm{RMSD}=3.885 e-09 \backslash \mathrm{RMSF}=5.363 e-06 \backslash \mathrm{Dipole}=-0.1603358,-0.3765946,0$ $.8095469 \backslash \mathrm{PG}=\mathrm{C} 02 \quad[\mathrm{X}(\mathrm{C} 2 \mathrm{H} 6 \mathrm{~S} 2)] \backslash \backslash @$

\section{R-R Products}

Product - Product 1-) $\mathrm{CH} 3 \mathrm{CH} 2 \mathrm{CH} 2-\mathrm{CH} 2 \mathrm{CH} 2 \mathrm{CH} 3$

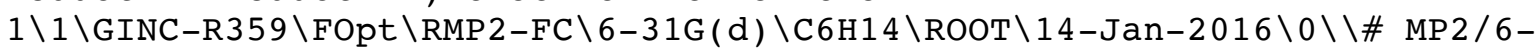
$31 \mathrm{G}(\mathrm{d})$ SCF=Tight INT(grid=ultrafine) OPT IOP(2/17=4) maxdisk=281857228

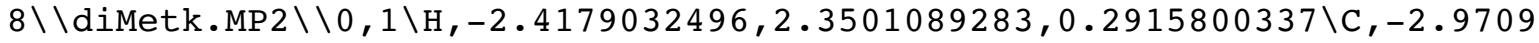
$621651,2.1713609835,1.2189690824 \backslash \mathrm{H},-2.3556422161,1.5281464362,1.855782$ $9059 \backslash \mathrm{H},-3.0917628501,3.1319013534,1.7285253208 \backslash \mathrm{C},-4.3198392269,1.51817$ $23905,0.9294196564 \backslash \mathrm{C},-4.1862525489,0.1738103151,0.2176625908 \backslash \mathrm{H},-4.9308$ $895399,2.1912351267,0.3149459178 \backslash \mathrm{H},-4.8690537529,1.3748866867,1.868465$ $1925 \backslash \mathrm{H},-3.574967376,-0.5014122776,0.8318830099 \backslash \mathrm{H},-3.6368586761,0.31566$ 
$90386,-0.7230309356 \backslash \mathrm{C},-5.5293248236,-0.4898552841,-0.0776233789 \backslash \mathrm{H},-6.0$ $787205482,-0.631800549,0.8630264065 \backslash \mathrm{H},-6.1406119518,0.1852821337,-0.69$ $18901395 \backslash \mathrm{C},-5.3955627582,-1.8342304873,-0.789394324 \backslash \mathrm{C},-6.7443822469,-2$ $.4876098564,-1.0790462912 \backslash \mathrm{H},-4.8464089087,-1.6908720185,-1.7284134958 \backslash$ $\mathrm{H},-4.7845722314,-2.5072322106,-0.1748718567 \backslash \mathrm{H},-6.6234813694,-3.4481518$ $729,-1.5886073829 \backslash \mathrm{H},-7.297382862,-2.6663728845,-0.1516696873 \backslash \mathrm{H},-7.3596$ $437586,-1.8444122005,-1.7158691188 \backslash \backslash$ Version=ES64L-G09RevD .01\State=1-A ' $\backslash \mathrm{HF}=-235.3670154 \backslash \mathrm{MP} 2=-236.1574348 \backslash \mathrm{RMSD}=3.558 e-09 \backslash \mathrm{RMSF}=1.439 e-05 \backslash \mathrm{Dipol}$ $e=0.0000063,0.0000161,0.0000082 \backslash P G=C S \quad[S G(C 6 H 2), X(H 12)] \backslash \backslash @$

Product - Product 2-) $\mathrm{CH} 3 \mathrm{CH} 2 \mathrm{C}(\mathrm{CH} 3) 2-\mathrm{C}(\mathrm{CH} 3) 2 \mathrm{CH} 2 \mathrm{CH} 3$

$1 \backslash 1 \backslash G I N C-R 359 \backslash F O p t \backslash R M P 2-F C \backslash 6-31 G(d) \backslash C 10 H 22 \backslash R O O T \backslash 15-J a n-2016 \backslash 0 \backslash \backslash \#$ MP $2 / 6$ $-31 \mathrm{G}(\mathrm{d}) \mathrm{SCF}=\mathrm{Tight}$ INT(grid=ultrafine) OPT IOP(2/17=4) maxdisk=25501368 $32 \backslash \backslash \mathrm{diMetk} . \mathrm{MP} 2 \backslash \backslash 0,1 \backslash \mathrm{H},-2.9302980724,2.1802766749,1.0101363204 \backslash \mathrm{C},-3.916$ $9188483,1.7068961375,1.0327418316 \backslash \mathrm{C},-3.8705161365,0.3848423406,0.26247$ $93924 \backslash \mathrm{H},-4.6276278292,2.4161629907,0.5999379814 \backslash \mathrm{H},-4.1842658252,1.5583$ $391526,2.0815374669 \backslash \mathrm{H},-3.1552242812,-0.2713025124,0.7720658427 \backslash \mathrm{H},-3.45$ $23830755,0.5814141553,-0.731219988 \backslash \mathrm{C},-5.219992161,-0.3547349166,0.0913$ $02574 \backslash \mathrm{C},-5.9635062126,-0.3634374448,1.4358181942 \backslash \mathrm{C},-6.0758478283,0.434$ $0177664,-0.9123792451 \backslash \mathrm{C},-4.9718399203,-1.8310266337,-0.4174281211 \backslash \mathrm{C},-6$ $.292062186,-2.4421539557,-0.9474284887 \backslash \mathrm{C},-3.9414703033,-1.8516132401,-$ $1.5575623795 \backslash \mathrm{C},-4.4302257308,-2.7112911029,0.7193854839 \backslash \mathrm{C},-6.288437937$ $1,-3.9537918147,-1.1886962711 \backslash \mathrm{H},-7.1057769029,-2.2196922922,-0.2472645$ $216 \backslash \mathrm{H},-6.5528856598,-1.9452890282,-1.8887005671 \backslash \mathrm{H},-7.2360470194,-4.255$ $2703692,-1.6460469468 \backslash \mathrm{H},-5.4876411121,-4.265568621,-1.8647239509 \backslash \mathrm{H},-6$. $181829759,-4.5148781049,-0.25738765 \backslash \mathrm{H},-7.1011193479,0.0575428368,-0.95$ $36733051 \backslash \mathrm{H},-6.1322721638,1.4852426388,-0.6149052327 \backslash \mathrm{H},-5.6576467489,0$. $4005038054,-1.9232415374 \backslash \mathrm{H},-6.8517209568,-1.0009759304,1.4036931515 \backslash \mathrm{H}$, $-5.325552864,-0.7128412407,2.2529765178 \backslash \mathrm{H},-6.3019009451,0.6458888559,1$ $.6878536011 \backslash \mathrm{H},-3.8741910637,-2.8557791444,-1.9863721639 \backslash \mathrm{H},-4.211623355$ $,-1.1660876832,-2.3668401153 \backslash \mathrm{H},-2.9416246346,-1.5867380312,-1.20433052$ $64 \backslash \mathrm{H},-4.080081427,-3.6688268417,0.3227763794 \backslash \mathrm{H},-3.580513185,-2.2432805$ $039,1.2247297371 \backslash \mathrm{H},-5.1958575072,-2.9240099432,1.4713005365 \backslash \backslash$ Version=E S64L-G09RevD. $01 \backslash$ State $=1-A \backslash H F=-391.4761859 \backslash M P 2=-392.8169834 \backslash \mathrm{RMSD}=5.580 \mathrm{e}$ $-09 \backslash \mathrm{RMSF}=2.137 e-06 \backslash \mathrm{Dipole}=-0.0002982,-0.0013065,0.0036382 \backslash \mathrm{PG}=\mathrm{C} 01] \mathrm{X}(\mathrm{C} 1$ $0 \mathrm{H} 22$ ) $] \backslash \backslash$ Q

Product - Product 3-) $\mathrm{CH} 3 \mathrm{CH} 2 \mathrm{CH} 2 \mathrm{CH} 2-\mathrm{CH} 2 \mathrm{CH} 2 \mathrm{CH} 2 \mathrm{CH} 3$

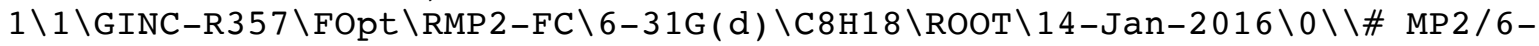
$31 \mathrm{G}(\mathrm{d}) \mathrm{SCF}=\mathrm{Tight}$ INT(grid=ultrafine) OPT IOP(2/17=4) maxdisk=416074956 $8 \backslash \backslash$ diMetk. MP $2 \backslash \backslash 0,1 \backslash \mathrm{H},-2.4227424978,2.3373872388,0.2851405009 \backslash \mathrm{C},-2.9759$ $332359,2.1584497829,1.2123823795 \backslash \mathrm{H},-2.3604851638,1.5154682803,1.849261$ $1475 \backslash \mathrm{H},-3.0970230438,3.119010309,1.721960485 \backslash \mathrm{C},-4.3243480982,1.5042765$ $554,0.9222969156 \backslash \mathrm{C},-4.1899915424,0.1597929722,0.2104456719 \backslash \mathrm{H},-4.935596$ $1691,2.1770511095,0.307726847 \backslash \mathrm{H},-4.8737637561,1.3607419425,1.861171999$ $7 \backslash \mathrm{H},-3.5784124665,-0.5150700814,0.8247658238 \backslash \mathrm{H},-3.6402985063,0.3019470$ $654,-0.7300266223 \backslash \mathrm{C},-5.5327502583,-0.5048665185,-0.08537528 \backslash \mathrm{H},-6.08215$ $94269,-0.6471453598,0.8551444933 \backslash \mathrm{H},-6.144053213,0.1699740536,-0.699842$ $567 \backslash \mathrm{C},-5.3961474874,-1.8492352741,-0.7972555907 \backslash \mathrm{C},-6.7388967254,-2.513$ $5865807,-1.0929149742 \backslash \mathrm{H},-4.846893116,-1.7070488447,-1.7378851969 \backslash \mathrm{H},-4$. $7849951285,-2.5242237245,-0.1827925833 \backslash \mathrm{C},-6.6042003697,-3.8577327167,-$ $1.8046024203 \backslash \mathrm{H},-7.2884068183,-2.6557678328,-0.152333367 \backslash \mathrm{H},-7.350301011$ $6,-1.8386430438,-1.7073306571 \backslash \mathrm{C},-7.9526075842,-4.5114456338,-2.0944463$ $033 \backslash \mathrm{H},-6.0549296512,-3.7141751773,-2.7435618814 \backslash \mathrm{H},-5.9930908712,-4.530$ $568402,-1.1899567659 \backslash \mathrm{H},-7.8313337781,-5.4719705856,-2.6040130388 \backslash \mathrm{H},-8$. $505723125,-4.6903344624,-1.1671423067 \backslash \mathrm{H},-8.5679829236,-3.8683829658,-2$ $.7313248736 \backslash \backslash$ Version=ES64L-G09RevD.01\State=1-A ' $\backslash \mathrm{HF}=-313.4361489 \backslash \mathrm{MP} 2=-$ $314.4888608 \backslash \mathrm{RMSD}=6.333 \mathrm{e}-09 \backslash \mathrm{RMSF}=2.455 \mathrm{e}-05 \backslash \mathrm{Dipole}=-0.000021,-0.0001292$, $-0.0000671 \backslash \mathrm{PG}=\mathrm{CS} \quad[\mathrm{SG}(\mathrm{C} 8 \mathrm{H} 2), \mathrm{X}(\mathrm{H} 16)] \backslash \backslash @$ 
Product - Product $4-)$ CH $3 \mathrm{CH} 2 \mathrm{CH} 2 \mathrm{C}(\mathrm{CH} 3) 2-\mathrm{C}(\mathrm{CH} 3) 2 \mathrm{CH} 2 \mathrm{CH} 2 \mathrm{CH} 3$

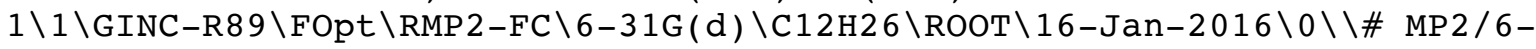
$31 \mathrm{G}$ (d) SCF=Tight INT(grid=ultrafine) OPT IOP $(2 / 17=4)$ maxdisk=416074956 $8 \backslash \backslash$ diMetk.MP $2 \backslash \backslash 0,1 \backslash \mathrm{H},-2.4494062168,2.3653568826,0.2247866679 \backslash \mathrm{C},-2.9264$ $607541,2.1208833038,1.1790498503 \backslash \mathrm{H},-2.2843967447,1.402118623,1.6982618$ $888 \backslash \mathrm{H},-2.9630972518,3.0340651846,1.7808092906 \backslash \mathrm{C},-4.3230721845,1.542012$ $3891,0.9584631435 \backslash \mathrm{C},-4.2658687298,0.2471823019,0.1428170539 \backslash \mathrm{H},-4.94513$ $17073,2.2903606447,0.4544925409 \backslash \mathrm{H},-4.7891146585,1.3532119562,1.9311328$ $956 \backslash \mathrm{H},-3.693372276,-0.4816197881,0.7307825552 \backslash \mathrm{H},-3.6735479935,0.444297$ $27,-0.7596860306 \backslash \mathrm{C},-5.620354977,-0.3713784245,-0.2808174316 \backslash \mathrm{C},-6.58198$ $99958,-0.3522597832,0.9173222677 \backslash C,-6.2274249237,0.510975121,-1.383212$ $5061 \backslash \mathrm{C},-5.4055623999,-1.8502552674,-0.7986721579 \backslash \mathrm{C},-6.6602791261,-2.33$ $41074556,-1.5656580108 \backslash \mathrm{C},-4.2015357273,-1.9264258845,-1.7509662558 \backslash \mathrm{C},-$ $5.1337273571,-2.8009134935,0.3773406148 \backslash \mathrm{C},-6.7551831516,-3.8344009848$, $-1.8575791524 \backslash \mathrm{H},-7.5648021872,-2.053669725,-1.0108625031 \backslash \mathrm{H},-6.71206308$ $92,-1.8013133803,-2.5235997859 \backslash C,-7.9583725299,-4.1520973589,-2.743962$ $5892 \backslash \mathrm{H},-5.8436905232,-4.1918661008,-2.3498870629 \backslash \mathrm{H},-6.8437679366,-4.39$ $46325792,-0.9209732813 \backslash \mathrm{H},-8.0409381311,-5.2263164082,-2.935258051 \backslash \mathrm{H},-8$ $.8888446337,-3.8228541795,-2.2703915616 \backslash \mathrm{H},-7.8776975532,-3.6433139529$, $-3.7096736487 \backslash \mathrm{H},-7.2557252647,0.2204604586,-1.6133777532 \backslash \mathrm{H},-6.25364973$ $8,1.555201238,-1.0579291885 \backslash \mathrm{H},-5.6445279675,0.4694671845,-2.3086255497$ $\backslash \mathrm{H},-7.5003817089,-0.9087426213,0.7082485059 \backslash \mathrm{H},-6.1247301071,-0.7785515$ $91,1.8150173904 \backslash \mathrm{H},-6.8749033415,0.675690201,1.1505695193 \backslash \mathrm{H},-4.13934920$ $52,-2.9219383214,-2.2004045528 \backslash \mathrm{H},-4.2783190726,-1.2014134194,-2.567270$ $1489 \backslash \mathrm{H},-3.2588944229,-1.7517431552,-1.2259119116 \backslash \mathrm{H},-4.8010936732,-3.77$ $50598709,0.0068330549 \backslash \mathrm{H},-4.3447894128,-2.419467477,1.0322492936 \backslash \mathrm{H},-6.0$ $283293261,-2.9651575365,0.9851056005 \backslash \backslash$ Version=ES64L-G09RevD.01 \State $=1$ $-\mathrm{A} \backslash \mathrm{HF}=-469.5444558 \backslash \mathrm{MP} 2=-471.1489398 \backslash \mathrm{RMSD}=2.470 \mathrm{e}-09 \backslash \mathrm{RMSF}=1.151 \mathrm{e}-06 \backslash \mathrm{Dipo}$ $l e=-0.0059292,-0.008008,0.0203978 \backslash P G=C 01 \quad[X(C 12 \mathrm{H} 26)] \backslash \backslash @$

Product - Product 5-) $\mathrm{CH} 3 \mathrm{OCH} 2-\mathrm{CH} 2 \mathrm{OCH} 3$

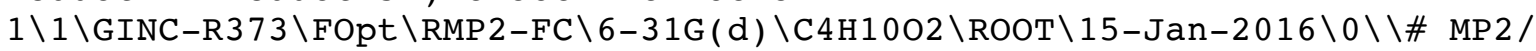
6-31G(d) SCF=Tight INT (grid=ultrafine) OPT IOP $(2 / 17=4$ ) maxdisk=1476395 $008 \backslash \backslash d i M e t k . M P 2 \backslash \backslash 0,1 \backslash \mathrm{H},-2.4946560568,0.723421182,1.0792578681 \backslash \mathrm{C},-2.956$ $4049522,1.6821659262,1.3262261069 \backslash \mathrm{H},-2.9424168108,1.8365689075,2.40643$ $48271 \backslash \mathrm{H},-2.3840056505,2.483034711,0.839101842 \backslash 0,-4.3269288556,1.731058$ $9676,0.9400182667 \backslash \mathrm{C},-4.5228285208,1.7605663814,-0.4638103106 \backslash \mathrm{H},-5.5497$ $715187,2.1129810145,-0.6082959598 \backslash \mathrm{H},-3.8384498999,2.4768617312,-0.9430$ $817932 \backslash \mathrm{C},-4.3769642942,0.4099072166,-1.134778141 \backslash \mathrm{H},-4.9137930161,-0.34$ $71673899,-0.5429614498 \backslash \mathrm{H},-4.8335446479,0.445220492,-2.1388877995 \backslash \mathrm{O},-2$. $9982461284,0.0833138997,-1.2388818394 \backslash \mathrm{C},-2.8115379318,-1.1944533665,-1$ $.8263557896 \backslash \mathrm{H},-1.7354211001,-1.3634800974,-1.8789794031 \backslash \mathrm{H},-3.275501214$ $9,-1.9853049632,-1.2214939788 \backslash \mathrm{H},-3.2351594012,-1.2330436125,-2.8395364$ 459\\Version=ES64L-G09RevD.01 \State $=1-\mathrm{A} \backslash \mathrm{HF}=-306.97434 \backslash \mathrm{MP} 2=-307.8519868$ $\backslash \mathrm{RMSD}=5.099 \mathrm{e}-09 \backslash \mathrm{RMSF}=6.774 \mathrm{e}-06 \backslash \mathrm{Dipole}=0.0893523,-0.41396,-0.55291 \backslash \mathrm{PG}=\mathrm{C}$ $01[\mathrm{X}(\mathrm{C} 4 \mathrm{H} 10 \mathrm{O} 2)] \backslash \backslash @$

Product - Product 6-) $\mathrm{CH} 3 \mathrm{CH} 2 \mathrm{OCH} 2-\mathrm{CH} 2 \mathrm{OCH} 2 \mathrm{CH} 3$

$1 \backslash 1 \backslash$ GINC-R3 90 $\backslash$ FOpt $\backslash$ RMP 2-FC $\backslash 6-31 \mathrm{G}(\mathrm{d}) \backslash \mathrm{C} 6 \mathrm{H} 14 \mathrm{O} 2 \backslash \mathrm{ROOT} \backslash 15-J a n-2016 \backslash 0 \backslash \backslash \#$ MP2 / 6-31G(d) SCF=Tight INT (grid=ultrafine) OPT IOP $(2 / 17=4$ ) maxdisk=1744830 $464 \backslash \backslash$ diMetk.MP $2 \backslash \backslash 0,1 \backslash \mathrm{H},-3.6704452985,1.7586363986,0.8396777051 \backslash \mathrm{C},-2.62$ $27051079,2.0748840938,0.887964717 \backslash \mathrm{H},-2.3275089395,2.4543696207,-0.1018$ $654014 \backslash \mathrm{C},-2.4994935821,3.1918100612,1.9042043958 \backslash 0,-1.8903205896,0.907$ $2569043,1.2235273345 \backslash \mathrm{C},-0.4869526285,0.9980968322,0.9642877382 \backslash \mathrm{H},-0.00$ $99383745,1.6938847897,1.6618816699 \backslash \mathrm{H},-3.3095448586,3.9248800726,1.7486$ $646206 \backslash \mathrm{H},-2.6083721397,2.7728936193,2.9161270572 \backslash 0,-1.2353687538,3.823$ $4856595,1.7531622939 \backslash \mathrm{H},-0.3244787527,1.3891281516,-0.0514893437 \backslash \mathrm{C}, 0.07$ $81854986,-0.3997076785,1.1032105138 \backslash \mathrm{H},-0.1053949848,-0.7810687945,2.11$ $02987172 \backslash \mathrm{H},-0.3973158253,-1.0734099063,0.3870418925 \backslash \mathrm{H}, 1.157221854,-0.3$ $964031917,0.9225214445 \backslash \mathrm{C},-1.0362029322,4.8527693313,2.7161373296 \backslash \mathrm{C}, 0.3$ 
$269359272,5.4606933992,2.4644438156 \backslash \mathrm{H},-1.8285803638,5.6118713483,2.622$ $0458113 \backslash \mathrm{H},-1.0996931726,4.4347412121,3.7325572884 \backslash \mathrm{H}, 0.5268682698,6.264$ $4700644,3.1787742707 \backslash \mathrm{H}, 1.103077538,4.6992355635,2.5677127669 \backslash \mathrm{H}, 0.37470$ $62166,5.8699554487,1.4531353624 \backslash \backslash$ Version=ES64L-G09RevD . $01 \backslash$ State $=1-A \backslash H F$ $=-385.0540946 \backslash M P 2=-386.1946252 \backslash \mathrm{RMSD}=8.693 e-09 \backslash \mathrm{RMSF}=5.130 \mathrm{e}-06 \backslash \mathrm{Dipole}=-0$ $.0394942,0.6310403,0.1928884 \backslash \mathrm{PG}=\mathrm{C} 01[\mathrm{X}(\mathrm{C} 6 \mathrm{H} 14 \mathrm{O} 2)] \backslash \backslash \mathrm{a}$

Product - Product 7-) $\mathrm{CH} 3 \mathrm{OC}(\mathrm{CH} 3) 2-\mathrm{C}(\mathrm{CH} 3) 20 \mathrm{CH} 3$

$1 \backslash 1 \backslash G I N C-R 2738 \backslash F O p t \backslash R M P 2-F C \backslash 6-31 G(d) \backslash C 8 H 1802 \backslash R O O T \backslash 15-J a n-2016 \backslash 0 \backslash \backslash \#$ MP 2 /6-31G(d) SCF=Tight INT(grid=ultrafine) OPT IOP(2/17=4) maxdisk=228170 $1376 \backslash \backslash$ diMetk.MP $2 \backslash \backslash 0,1 \backslash \mathrm{H},-2.096356624,2.333823831,0.6961078156 \backslash \mathrm{C},-3.024$ $4995979,1.7749163013,0.5650027195 \backslash \mathrm{H},-3.5999155329,2.2339630156,-0.2470$ $01765 \backslash \mathrm{H},-2.7779975803,0.7432946606,0.289008565 \backslash 0,-3.6959257254,1.85390$ $20454,1.8143488233 \backslash \mathrm{C},-4.9698147327,1.182596263,1.896127233 \backslash \mathrm{C},-5.421960$ $0943,1.458368717,3.3634845154 \backslash \mathrm{C},-4.8123430107,-0.3178646124,1.64793816$ $67 \backslash \mathrm{H},-5.7176392417,-0.8369713349,1.9674242474 \backslash \mathrm{H},-4.6577842729,-0.52333$ $17065,0.5849601543 \backslash \mathrm{H},-3.9571311737,-0.7144169401,2.2000789442 \backslash 0,-6.695$ $9762764,0.7872948022,3.4451792198 \backslash \mathrm{C},-4.4273336086,0.8630864533,4.36055$ $47742 \backslash \mathrm{C},-5.5793042322,2.9588419113,3.6117130365 \backslash \mathrm{C},-5.9645155203,1.7777$ $648092,0.8990552692 \backslash \mathrm{H},-5.7411306741,1.4473558373,-0.1192542372 \backslash \mathrm{H},-5.92$ $75260168,2.8694163495,0.9194969104 \backslash \mathrm{H},-6.9740491531,1.4481699681,1.1506$ $797943 \backslash \mathrm{C},-7.3666793176,0.8649664986,4.6949959854 \backslash \mathrm{H},-8.295642802,0.3075$ $497723,4.5633567139 \backslash \mathrm{H},-6.7914674527,0.4036874038,5.5058795276 \backslash \mathrm{H},-7.611$ $6555981,1.8964193071,4.9729715094 \backslash \mathrm{H},-5.733966524,3.1642894503,4.674679$ $302 \backslash \mathrm{H},-4.673937137,3.4779023443,3.2923512649 \backslash \mathrm{H},-6.4344323185,3.3554565$ $364,3.0594936392 \backslash \mathrm{H},-4.6506478696,1.1935740503,5.3788548081 \backslash \mathrm{H},-4.464442$ $4361,-0.2285654754,4.3401702075 \backslash \mathrm{H},-3.4177704766,1.1925447415,4.1088718$ $554 \backslash \backslash$ Version=ES6 4L-G09RevD.01 \State $=1-A \backslash H F=-463.110829 \backslash M P 2=-464.533212$ $6 \backslash \mathrm{RMSD}=7.420 \mathrm{e}-09 \backslash \mathrm{RMSF}=1.184 \mathrm{e}-05 \backslash \mathrm{Dipole}=0.0002937,-0.0005349,0.0001919 \backslash$ $\mathrm{PG}=\mathrm{C} 01 \quad[\mathrm{X}(\mathrm{C} 8 \mathrm{H} 1802)] \backslash \backslash @$

Product - Product 8-) CH3CH2OC( CH3 ) 2-C(CH3) 2OCH2 CH 3

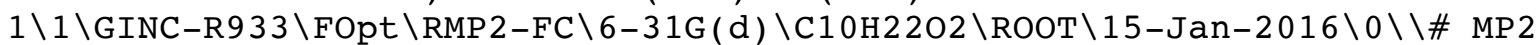
/6-31G(d) SCF=Tight INT(grid=ultrafine) OPT IOP(2/17=4) maxdisk=630823 $3216 \backslash \backslash$ diMetk.MP2 $\backslash \backslash 0,1 \backslash \mathrm{H},-2.6689665542,0.7509304872,1.3257759904 \backslash \mathrm{C},-3.0$ $966745296,1.7269368485,1.5902136878 \backslash \mathrm{H},-3.2349113856,1.7493905387,2.679$ $1748672 \backslash \mathrm{C},-2.1712097688,2.8443569194,1.1528431345 \backslash 0,-4.3337762632,1.94$ $06051786,0.9152659281 \backslash \mathrm{C},-5.3912934322,0.9929921234,1.1721554373 \backslash \mathrm{C},-4.9$ $795968756,-0.4145067624,0.7395485509 \backslash \mathrm{C},-6.5638219449,1.5242153852,0.28$ $99139061 \backslash \mathrm{H},-2.033677514,2.8152347819,0.0696502188 \backslash \mathrm{H},-2.5990614226,3.81$ $27130763,1.4217284032 \backslash \mathrm{H},-1.1938784737,2.7453197502,1.6345862591 \backslash 0,-7.6$ $213310291,0.576587085,0.5467817379 \backslash \mathrm{C},-6.9755248728,2.9317102728,0.7225$ $248311 \backslash \mathrm{C},-6.1761331616,1.5214214392,-1.1890677907 \backslash \mathrm{H},-5.8631232502,-1.0$ $53670121,0.6917910882 \backslash \mathrm{H},-4.4982147805,-0.3929549044,-0.240943091 \backslash \mathrm{H},-4$. $2780326408,-0.8525292919,1.4550373097 \backslash \mathrm{C},-5.7789760055,0.9957942843,2.6$ $511380489 \backslash \mathrm{H},-5.0292791082,0.4728301278,3.2515072335 \backslash \mathrm{H},-5.8647745188,2$. $0180097944,3.0269987487 \backslash \mathrm{H},-6.7354031408,0.4852452637,2.7776964404 \backslash \mathrm{C},-8$ $.8584718926,0.7903239544,-0.1280728008 \backslash \mathrm{C},-9.7838707318,-0.3272120511,0$ $.30914097 \backslash \mathrm{H},-9.286208817,1.7662653201,0.1365580174 \backslash \mathrm{H},-8.7202830365,0.7$ $680700205,-1.2170440861 \backslash \mathrm{H},-10.7612292246,-0.2281273881,-0.1725372328 \backslash \mathrm{H}$ $,-9.3559934785,-1.295503123,0.0400622153 \backslash \mathrm{H},-9.9213523008,-0.2982889049$ $, 1.3923456674 \backslash \mathrm{H},-6.9258390223,2.0443709725,-1.7894385158 \backslash \mathrm{H},-5.21971516$ $34,2.031987419,-1.3156239719 \backslash \mathrm{H},-6.09031728,0.4992064361,-1.5649251468 \backslash$ $\mathrm{H},-7.6770752474,3.3697407341,0.0070273754 \backslash \mathrm{H},-7.4569226486,2.9101559085$ $, 1.703008957 \backslash \mathrm{H},-6.0919974836,3.5708714253,0.7703016118 \backslash \backslash$ Version=ES $64 \mathrm{~L}-$ G09RevD.01\State $=1-A \backslash H F=-541.1900608 \backslash M P 2=-542.8762551 \backslash \mathrm{RMSD}=9.410 \mathrm{e}-09 \backslash \mathrm{R}$ $\mathrm{MSF}=4.344 \mathrm{e}-07 \backslash \mathrm{Dipole}=-0.0000145,0.0000267,0.0000365 \backslash \mathrm{PG}=\mathrm{C} 01] \mathrm{X}(\mathrm{C} 10 \mathrm{H} 22 \mathrm{O} 2$ )$] \backslash \backslash Q$

Product - Product 9-) HOCH2CH2-CH2CH2OH 


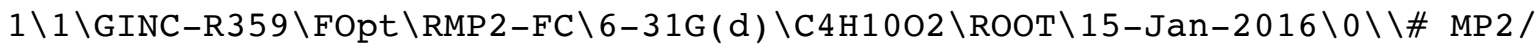
6-31G(d) SCF=Tight INT(grid=ultrafine) OPT IOP $(2 / 17=4)$ maxdisk=6710886 $40 \backslash \backslash$ diMetk.MP $2 \backslash \backslash 0,1 \backslash 0,-3.2240428364,3.0179560331,0.717988171 \backslash \mathrm{H},-2.3345$ $815808,2.6941203457,0.9646860844 \backslash \mathrm{C},-4.1408078885,2.0630645712,1.230838$ $4328 \backslash \mathrm{C},-4.1311635808,0.738461453,0.4701787156 \backslash \mathrm{H},-5.1305568249,2.519218$ $0784,1.1338626068 \backslash \mathrm{H},-3.9737575835,1.8785530412,2.3050774829 \backslash \mathrm{H},-5.04847$ $79033,0.1888676067,0.7209909077 \backslash \mathrm{H},-4.1850722493,0.9730211832,-0.599753$ $7544 \backslash \mathrm{C},-2.94241059,-0.1909057365,0.7319188067 \backslash \mathrm{H},-2.9303423901,-0.49143$ $11496,1.7904161987 \backslash \mathrm{H},-3.0928704851,-1.1167149677,0.1610240845 \backslash \mathrm{C},-1.565$ $0084151,0.3501060922,0.3680689245 \backslash 0,-1.0883269006,1.3647212138,1.27101$ $98735 \backslash \mathrm{H},-0.8357557861,-0.4694494143,0.329785976 \backslash \mathrm{H},-1.5823665237,0.8321$ $798173,-0.6121498315 \backslash \mathrm{H},-1.0422544618,0.9674958324,2.1579993207 \backslash \backslash$ Versio $\mathrm{n}=\mathrm{ES} 64 \mathrm{~L}-\mathrm{G} 09$ RevD. $01 \backslash$ State $=1-\mathrm{A} \backslash \mathrm{HF}=-306.9919478 \backslash \mathrm{MP} 2=-307.878185 \backslash \mathrm{RMSD}=3.84$ $9 e-09 \backslash \mathrm{RMSF}=1.030 \mathrm{e}-05 \backslash \mathrm{Dipole}=0.1677818,-1.3850643,0.4742442 \backslash \mathrm{PG}=\mathrm{C} 01 \quad[\mathrm{X}(\mathrm{C}$ $4 \mathrm{H} 10 \mathrm{O} 2)] \backslash \backslash @$

Product - Product $10-)$ HOCH $2 \mathrm{C}(\mathrm{CH} 3) 2-\mathrm{C}(\mathrm{CH} 3) 2 \mathrm{CH} 2 \mathrm{OH}$

$1 \backslash 1 \backslash G I N C-R 2486 \backslash F O p t \backslash R M P 2-F C \backslash 6-31 G(d) \backslash C 8 H 18 O 2 \backslash R O O T \backslash 16-J a n-2016 \backslash 0 \backslash \backslash \#$ MP2 /6-31G(d) SCF=Tight INT(grid=ultrafine) OPT IOP $(2 / 17=4)$ maxdisk $=416074$ $9568 \backslash \backslash$ dimetk.MP $2 \backslash \backslash 0,1 \backslash 0,-2.8689481103,2.2567474266,0.1566882054 \backslash \mathrm{H},-2.3$ $034463784,1.8359943975,0.8333331419 \backslash \mathrm{C},-4.1862395862,2.1889791854,0.681$ $6303368 \backslash \mathrm{C},-4.9275148042,0.8616046641,0.4158038862 \backslash \mathrm{H},-4.751517742,2.979$ $8925953,0.1745114946 \backslash \mathrm{H},-4.1986282249,2.4276617056,1.7554580854 \backslash \mathrm{C},-4.76$ $98265784,0.5465187119,-1.0772101922 \backslash C,-6.4120586338,1.1566364215,0.686$ $0577485 \backslash \mathrm{H},-7.0337343021,0.2597732807,0.615172817 \backslash \mathrm{H},-6.5683597006,1.602$ $127355,1.6735280464 \backslash \mathrm{H},-6.782032925,1.8698290472,-0.0579097916 \backslash \mathrm{H},-5.445$ $7232471,-0.2525872102,-1.3941500105 \backslash \mathrm{H},-5.0148173259,1.4400794567,-1.66$ $1019978 \backslash \mathrm{H},-3.7457322894,0.2711106456,-1.3359946505 \backslash \mathrm{C},-4.4315627941,-0$. $3291263533,1.3113393308 \backslash C,-2.9026808699,-0.511100882,1.3047638014 \backslash \mathrm{C},-4$ $.8788490783,-0.1660683566,2.7717744721 \backslash \mathrm{C},-4.9891832897,-1.668967357,0$. $8011321078 \backslash \mathrm{O},-2.1586481478,0.4703168194,2.0453370951 \backslash \mathrm{H},-2.6726111785,-$ $1.5075641261,1.7087555328 \backslash \mathrm{H},-2.5036046418,-0.4637651776,0.2899866368 \backslash \mathrm{H}$ $,-2.4294229097,0.4093554185,2.9769207977 \backslash \mathrm{H},-4.7802397076,-2.4603515466$ $, 1.5294557331 \backslash \mathrm{H},-6.0719264064,-1.6309366741,0.6584582814 \backslash \mathrm{H},-4.53228206$ $34,-1.9631155303,-0.1480600464 \backslash \mathrm{H},-4.403710153,-0.9329571811,3.39756385$ $76 \backslash \mathrm{H},-4.6271616421,0.8163247387,3.1827831941 \backslash \mathrm{H},-5.9585152695,-0.298165$ $4747,2.8728310662 \backslash \backslash$ Version $=$ ES 64L-G09RevD. $01 \backslash$ State $=1-A \backslash H F=-463.1132606 \backslash$ MP2 $=-464.5463852 \backslash \mathrm{RMSD}=5.284 \mathrm{e}-09 \backslash \mathrm{RMSF}=5.352 \mathrm{e}-06 \backslash \mathrm{Dipole}=-0.5939173,-0.84$ $01508,0.908734 \backslash \mathrm{PG}=\mathrm{C} 01 \quad[\mathrm{X}(\mathrm{C} 8 \mathrm{H} 18 \mathrm{O} 2)] \backslash \backslash @$

Product - Product 11-) $\mathrm{CH} 3 \mathrm{OCH} 2 \mathrm{CH} 2-\mathrm{CH} 2 \mathrm{CH} 2 \mathrm{OCH} 3$

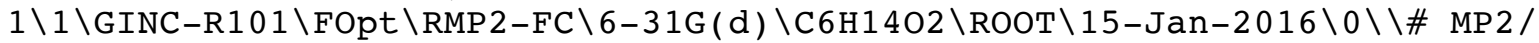
6-31G(d) SCF=Tight INT (grid=ultrafine) OPT IOP $(2 / 17=4)$ maxdisk=4429185 $024 \backslash \backslash$ diMetk.MP $2 \backslash \backslash 0,1 \backslash 0,-2.5385942353,2.3773373496,1.4061882126 \backslash \mathrm{C},-2.23$ $69508265,3.0721592079,2.6037363662 \backslash \mathrm{C},-3.8433261717,1.8113387251,1.4483$ $399146 \backslash \mathrm{C},-4.0948069088,1.0918923268,0.1377207587 \backslash \mathrm{H},-4.5947639237,2.600$ $9220309,1.6024436114 \backslash \mathrm{H},-3.9254066564,1.1101582458,2.2946593353 \backslash \mathrm{H},-5.12$ $67571461,0.7264202559,0.1438788094 \backslash \mathrm{H},-1.232561369,3.482294917,2.488900$ $498 \backslash \mathrm{H},-2.9463643764,3.8930119217,2.7806906225 \backslash \mathrm{H},-2.2569104371,2.400537$ $9207,3.4733758926 \backslash \mathrm{H},-3.4362973198,0.2181366116,0.0783336097 \backslash \mathrm{C},-3.86928$ $00849,1.9747968244,-1.0905447944 \backslash \mathrm{H},-2.8022190065,2.1969628286,-1.19326$ $75773 \backslash \mathrm{H},-4.1798445727,1.4284030248,-1.9881049743 \backslash \mathrm{C},-4.6194758544,3.290$ $8724356,-1.0295567148 \backslash 0,-5.9947372548,3.0127421664,-0.793429061 \backslash \mathrm{H},-4.2$ $167660565,3.9246338849,-0.2245600159 \backslash \mathrm{H},-4.5015396782,3.8457067162,-1.9$ $74489417 \backslash \mathrm{C},-6.7584905072,4.2040370207,-0.72004459 \backslash \mathrm{H},-7.7909307488,3.90$ $83915206,-0.5280611408 \backslash \mathrm{H},-6.4095758039,4.8545723517,0.0944748293 \backslash \mathrm{H},-6$. $7124014858,4.7690102472,-1.6615473785 \backslash \backslash$ Version=ES64L-G09RevD.01\State= $1-\mathrm{A} \backslash \mathrm{HF}=-385.0461134 \backslash \mathrm{MP} 2=-386.1881388 \backslash \mathrm{RMSD}=5.786 \mathrm{e}-09 \backslash \mathrm{RMSF}=5.088 \mathrm{e}-06 \backslash \mathrm{Dip}$ ole $=-0.1253836,0.5117573,0.3448403 \backslash P G=C 02 \quad[\mathrm{X}(\mathrm{C} 6 \mathrm{H} 14 \mathrm{O} 2)] \backslash \backslash @$ 
Product - Product $12-)$ CH3OCH $2 \mathrm{C}(\mathrm{CH} 3) 2-\mathrm{C}(\mathrm{CH} 3) 2 \mathrm{CH} 2 \mathrm{OCH} 3$

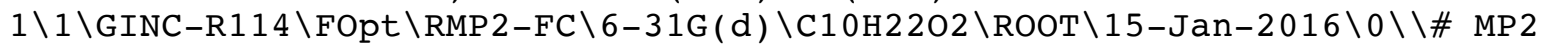
$16-31 \mathrm{G}(\mathrm{d}) \quad \mathrm{SCF}=\mathrm{Tight}$ INT(grid=ultrafine) OPT IOP $(2 / 17=4)$ maxdisk $=684510$ $4128 \backslash \backslash$ diMetk.MP $2 \backslash \backslash 0,1 \backslash 0,-3.7039335038,2.5198019358,0.3520072571 \backslash \mathrm{C},-3.0$ $261412452,1.544034087,1.1268201382 \backslash C,-5.1116986174,2.4486670652,0.5579$ $717628 \backslash \mathrm{C},-5.8471798221,3.5314300513,-0.2346191363 \backslash \mathrm{H},-5.4879001401,1.45$ $37826178,0.276203902 \backslash \mathrm{H},-5.3286002722,2.59281481,1.6304922651 \backslash \mathrm{C},-5.4118$ $213515,4.8996129624,0.3072023662 \backslash C,-7.3360074667,3.3471988292,0.109961$ $6483 \backslash \mathrm{H},-7.9314801875,4.1800371742,-0.2723899673 \backslash \mathrm{H},-7.7300846828,2.4162$ $00201,-0.2988365023 \backslash \mathrm{H},-7.4599061997,3.3352341353,1.1992413516 \backslash \mathrm{H},-5.876$ $6159281,5.7178683676,-0.2521862229 \backslash \mathrm{H},-5.7304531962,4.9927131992,1.3517$ $557582 \backslash \mathrm{H},-4.3279672146,5.0214611736,0.2754074333 \backslash \mathrm{H},-1.9611106844,1.657$ $1533118,0.9190180962 \backslash \mathrm{H},-3.205410377,1.6927032853,2.2006750367 \backslash \mathrm{H},-3.344$ $1833706,0.5271876041,0.8567032084 \backslash \mathrm{C},-5.5842561277,3.4175748095,-1.7826$ $550878 \backslash \mathrm{C},-4.2613851598,4.0900909469,-2.1913349907 \backslash \mathrm{C},-5.457535893,1.963$ $5314134,-2.2422292432 \backslash \mathrm{C},-6.7091726848,4.0782395378,-2.5908115534 \backslash 0,-6$. $6370897245,1.2209434341,-1.9481203961 \backslash \mathrm{H},-5.2868854168,1.9554658877,-3$. $332613804 \backslash \mathrm{H},-4.5832380468,1.4928953052,-1.7680766238 \backslash \mathrm{C},-6.5300540185,-$ $0.1124435136,-2.4198012697 \backslash \mathrm{H},-7.4621157141,-0.6167894877,-2.160641324 \backslash$ $\mathrm{H},-6.3919688081,-0.1399395967,-3.5095630489 \backslash \mathrm{H},-5.6879564824,-0.6374955$ $364,-1.9472731417 \backslash \mathrm{H},-4.0697669573,3.9028084873,-3.2544587214 \backslash \mathrm{H},-4.3172$ $632018,5.1736850001,-2.0605611389 \backslash \mathrm{H},-3.4212018516,3.708536928,-1.61023$ $27922 \backslash \mathrm{H},-6.4291105855,4.0986208748,-3.6502621267 \backslash \mathrm{H},-7.6475917281,3.528$ $4859013,-2.5028437599 \backslash \mathrm{H},-6.8742713601,5.1132833136,-2.2747129776 \backslash \backslash$ Vers ion $=\mathrm{ES} 64 \mathrm{~L}-\mathrm{G} 09 \mathrm{RevD} .01 \backslash \mathrm{State}=1-\mathrm{A} \backslash \mathrm{HF}=-541.1661042 \backslash \mathrm{MP} 2=-542.8567357 \backslash \mathrm{RMSD}=5$ $.716 \mathrm{e}-09 \backslash \mathrm{RMSF}=9.995 \mathrm{e}-06 \backslash \mathrm{Dipole}=0.2244755,-0.6604614,0.0867014 \backslash \mathrm{PG}=\mathrm{C} 02 \quad[$ $\mathrm{X}(\mathrm{C} 1 \mathrm{OH} 22 \mathrm{O} 2)] \backslash \backslash @$

Product - Product 13-) HOOCH2-CH2OOH

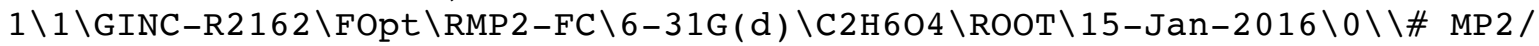
6-31G(d) SCF=Tight INT(grid=ultrafine) OPT IOP $(2 / 17=4)$ maxdisk=6710886 $40 \backslash \backslash$ diMetk.MP $2 \backslash \backslash 0,1 \backslash 0,-3.5991903821,2.0857169705,2.0624871291 \backslash \mathrm{H},-3.002$ $1431756,1.3898160691,2.4180062107 \backslash 0,-4.0836093605,1.3734946131,0.87585$ $63269 \backslash \mathrm{C},-3.1872587795,1.7128687175,-0.1852284287 \backslash \mathrm{H},-3.2021142619,2.795$ $4001387,-0.3616116676 \backslash \mathrm{H},-3.6186783277,1.1928681412,-1.0468497015 \backslash \mathrm{C},-1$. $7690930097,1.2326796231,0.0645034221 \backslash \mathrm{H},-1.2958069374,1.7683606491,0.89$ $19992816 \backslash \mathrm{O},-1.7059516318,-0.1758835627,0.2998473135 \backslash \mathrm{H},-1.1630873121,1$. $3736816924,-0.8375835439 \backslash \mathrm{O},-2.1829811584,-0.3988184998,1.6747406735 \backslash \mathrm{H}$, $-3.1188916632,-0.6131695522,1.4757439844 \backslash \backslash$ Version=ES64L-G09RevD.01\Sta $\mathrm{te}=1-\mathrm{A} \backslash \mathrm{HF}=-378.4334281 \backslash \mathrm{MP} 2=-379.4317758 \backslash \mathrm{RMSD}=9.326 \mathrm{e}-09 \backslash \mathrm{RMSF}=2.260 \mathrm{e}-05 \backslash$ Dipole $=0.2120471,0.1829476,-0.564752 \backslash P G=C 01 \quad[X(C 2 \mathrm{H} 604)] \backslash \backslash @$

Product - Product $14-)$ HOOC $(\mathrm{CH} 3) 2-\mathrm{C}(\mathrm{CH} 3) 200 \mathrm{H}$

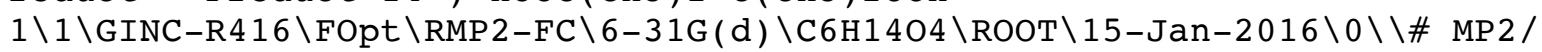
6-31G(d) SCF=Tight INT(grid=ultrafine) OPT IOP $(2 / 17=4)$ maxdisk=5502926 $848 \backslash \backslash$ diMetk.MP $2 \backslash \backslash 0,1 \backslash 0,-3.6090327085,1.5483134668,1.590196925 \backslash \mathrm{H},-2.701$ $1658681,1.2083575409,1.4185148248 \backslash 0,-4.1251343695,1.4514558017,0.21864$ $72935 \backslash C,-3.7274582159,2.627813244,-0.5364137821 \backslash C,-2.1563249966,2.7115$ $913688,-0.5938847054 \backslash \mathrm{C},-4.3719151578,3.8646454101,0.07509647 \backslash \mathrm{H},-4.0982$ $994634,4.7628158493,-0.4876055974 \backslash \mathrm{H},-5.4582391656,3.7495666156,0.03678$ $42365 \backslash \mathrm{H},-4.0757867679,3.9896898346,1.1160037213 \backslash 0,-1.5867477741,1.4209$ $928845,-0.2445852694 \backslash \mathrm{C},-1.6284796601,3.1473698509,-1.9542144454 \backslash \mathrm{C},-1.5$ $392729974,3.595435507,0.4918595874 \backslash \mathrm{C},-4.3854850458,2.3326682807,-1.885$ $7310999 \backslash \mathrm{H},-5.4494460064,2.1473710094,-1.7166541803 \backslash \mathrm{H},-4.2889922427,3.1$ $95150575,-2.5490196056 \backslash \mathrm{H},-3.9458485916,1.4641138292,-2.375409317 \backslash \mathrm{O},-2$. $0392916406,0.406259795,-1.2050069696 \backslash \mathrm{H},-2.9093054522,0.204772091,-0.79$ $05853567 \backslash \mathrm{H},-0.4537069664,3.4706887723,0.4615306568 \backslash \mathrm{H},-1.7690695629,4.6$ $457122236,0.298856532 \backslash \mathrm{H},-1.9002307479,3.3356594449,1.4866839671 \backslash \mathrm{H},-0.5$ $395820884,3.2255545707,-1.8994415986 \backslash \mathrm{H},-1.8828423629,2.4223269444,-2.7$ $265023899 \backslash \mathrm{H},-2.0328821472,4.1259800895,-2.2316838969 \backslash \backslash$ Version=ES $64 \mathrm{~L}-\mathrm{G} 0$ 
9RevD.01 \State $=1-\mathrm{A} \backslash \mathrm{HF}=-534.5713045 \backslash \mathrm{MP} 2=-536.1166161 \backslash \mathrm{RMSD}=9.750 \mathrm{e}-09 \backslash \mathrm{RMS}$ $\mathrm{F}=3.989 \mathrm{e}-06 \backslash \mathrm{Dipole}=-0.0623098,0.8945663,-0.4001979 \backslash \mathrm{PG}=\mathrm{C} 01 \quad[\mathrm{X}(\mathrm{C} 6 \mathrm{H} 14 \mathrm{O} 4)]$ $\backslash \backslash \mathrm{Q}$

Product - Product 15-) $\mathrm{CH} 30 \mathrm{OCH} 2-\mathrm{CH} 2 \mathrm{OOCH} 3$

$1 \backslash 1 \backslash G I N C-R 183 \backslash F O p t \backslash R M P 2-F C \backslash 6-31 G(d) \backslash C 4 H 1004 \backslash R O O T \backslash 15-J a n-2016 \backslash 0 \backslash \backslash \#$ MP2/ 6-31G(d) SCF=Tight INT (grid=ultrafine) OPT IOP $(2 / 17=4$ ) maxdisk=1476395 $008 \backslash \backslash$ diMetk.MP $2 \backslash \backslash 0,1 \backslash 0,-2.8971061807,1.8452396451,1.2161045175 \backslash \mathrm{C},-2.61$ $69764623,3.1939305886,1.5859597975 \backslash 0,-4.1912286725,1.5575518203,1.8650$ $605987 \backslash \mathrm{C},-3.987728318,0.3423995285,2.5790658434 \backslash \mathrm{H},-4.9788468227,0.1245$ $983342,2.9854954888 \backslash \mathrm{H},-1.6695480591,3.4157651347,1.0885234396 \backslash \mathrm{H},-3.397$ $73573,3.8654061236,1.2171176878 \backslash \mathrm{H},-2.5194848548,3.2877463666,2.6701267$ $449 \backslash \mathrm{H},-3.6961408234,-0.4610706745,1.8898306846 \backslash \mathrm{C},-2.9655432073,0.46079$ $43387,3.6922440811 \backslash \mathrm{H},-2.922509498,-0.4783199269,4.2595587797 \backslash \mathrm{H},-1.9747$ $699605,0.6804533029,3.2859758633 \backslash 0,-3.2060807422,1.5576005891,4.567995$ $355 \backslash 0,-4.4979787394,1.2578865204,5.2159296374 \backslash C,-5.2656423261,2.444132$ $7369,5.0217121879 \backslash \mathrm{H},-6.2150347027,2.2321181579,5.5196814301 \backslash \mathrm{H},-4.77890$ $60503,3.3009323464,5.4965730017 \backslash \mathrm{H},-5.4184398499,2.6386890675,3.9575068$ $609 \backslash \backslash$ Version=ES64L-G0 9RevD. 01 \State $=1-\mathrm{A} \backslash \mathrm{HF}=-456.4949663 \backslash \mathrm{MP} 2=-457.75125$ $54 \backslash \mathrm{RMSD}=4.286 \mathrm{e}-09 \backslash \mathrm{RMSF}=8.365 \mathrm{e}-06 \backslash \mathrm{Dipole}=-0.034494,0.17884,0.0115188 \backslash \mathrm{PG}$ $=\mathrm{C} 01[\mathrm{X}(\mathrm{C} 4 \mathrm{H} 1004)] \backslash \backslash @$

Product - Product 16-) $\mathrm{CH} 300 \mathrm{C}(\mathrm{CH} 3) 2-\mathrm{C}(\mathrm{CH} 3) 200 \mathrm{CH} 3$

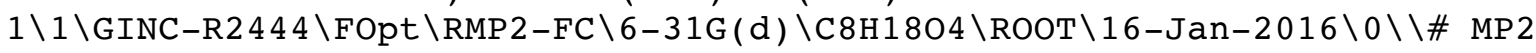
$16-31 \mathrm{G}(\mathrm{d}) \quad \mathrm{SCF}=\mathrm{Tight}$ INT(grid=ultrafine) OPT IOP $(2 / 17=4)$ maxdisk $=416074$ $9568 \backslash \backslash$ diMetk.MP $2 \backslash \backslash 0,1 \backslash 0,-3.1179490765,3.1569614394,1.1646622682 \backslash \mathrm{C},-1.8$ $687424408,2.4928500583,1.3223828021 \backslash 0,-3.9783435535,2.1357291244,0.522$ $5064256 \backslash \mathrm{C},-5.2414728795,2.0972129957,1.2303455228 \backslash \mathrm{C},-5.9883726556,3.46$ $3342492,1.0635370307 \backslash \mathrm{C},-4.9963063186,1.6819194913,2.675340093 \backslash \mathrm{H},-1.204$ $5642486,3.2609324299,1.7267401639 \backslash \mathrm{H},-1.9516444192,1.6543745449,2.01975$ $33503 \backslash \mathrm{H},-1.492510169,2.1418987395,0.35732914 \backslash \mathrm{H},-5.9429858395,1.5995773$ $477,3.2114840075 \backslash \mathrm{H},-4.4979547131,0.7076773716,2.679461402 \backslash \mathrm{H},-4.3566905$ $244,2.4013732563,3.1879696171 \backslash 0,-7.415469906,3.2365554924,0.9636547564$ $\backslash C,-5.7154614425,4.4735020597,2.1706421579 \backslash \mathrm{C},-5.6971824898,4.083471778$ $8,-0.3007127505 \backslash \mathrm{C},-5.9627725793,0.9832243345,0.4758354059 \backslash \mathrm{H},-5.3371431$ $006,0.0864371091,0.4883560855 \backslash \mathrm{H},-6.9135874948,0.7695793003,0.965000252$ $9 \backslash \mathrm{H},-6.1562193458,1.2612917942,-0.5615466638 \backslash \mathrm{H},-6.3485710026,4.9506820$ $257,-0.4401248478 \backslash \mathrm{H},-4.6550713588,4.4019447424,-0.3401438788 \backslash \mathrm{H},-5.8787$ $173253,3.3748438114,-1.1105252161 \backslash \mathrm{H},-6.25895075,5.3959162718,1.9441682$ $965 \backslash \mathrm{H},-6.0548330462,4.0987939848,3.137043672 \backslash \mathrm{H},-4.6484222614,4.6940432$ $941,2.2274012167 \backslash 0,-7.8849409491,2.6641883784,2.2472244078 \backslash \mathrm{C},-9.039085$ $1307,3.4374521507,2.557790437 \backslash \mathrm{H},-9.4370957579,2.974988085,3.4645994606$ $\backslash \mathrm{H},-8.7764068902,4.482349948,2.7460146148 \backslash \mathrm{H},-9.7749725743,3.3754410399$ , $1.7511489963 \backslash \backslash$ Version=ES64L-G09RevD.01 $\backslash$ State $=1-\mathrm{A} \backslash \mathrm{HF}=-612.6325531 \backslash \mathrm{MP} 2=$ $-614.4353705 \backslash \mathrm{RMSD}=5.731 \mathrm{e}-09 \backslash \mathrm{RMSF}=1.063 \mathrm{e}-05 \backslash \mathrm{Dipole}=0.1183053,0.1358402$ ， $0.582783 \backslash P G=C 02 \quad[X(C 8 H 1804)] \backslash \backslash @$

Product - Product 17-) HSCH2 $\mathrm{CH} 2-\mathrm{CH} 2 \mathrm{CH} 2 \mathrm{SH}$

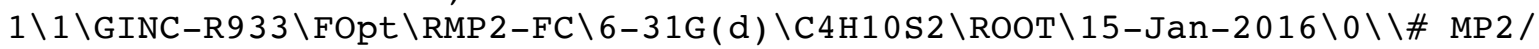
6-31G(d) SCF=Tight INT(grid=ultrafine) OPT IOP $(2 / 17=4)$ maxdisk=4966055 $936 \backslash \backslash d i M e t k . M P 2 \backslash \backslash 0,1 \backslash \mathrm{S},-2.8092179535,2.6805180795,1.2288090857 \backslash \mathrm{H},-2.18$ $46148779,1.4949968894,1.1517821069 \backslash \mathrm{C},-4.5003553224,2.0091716808,1.3369$ $38418 \backslash \mathrm{C},-4.9404196308,1.1691084346,0.1416696434 \backslash \mathrm{H},-5.1485850673,2.8823$ $540899,1.4503661773 \backslash \mathrm{H},-4.5968557286,1.4272852937,2.2575288627 \backslash \mathrm{H},-5.975$ $3363404,0.8592471915,0.3256576245 \backslash \mathrm{H},-4.3396377235,0.251472678,0.103790$ $2317 \backslash \mathrm{C},-4.8493502229,1.8665570689,-1.2146735473 \backslash \mathrm{H},-3.796832207,2.02642$ $94529,-1.4746058654 \backslash \mathrm{H},-5.2639966457,1.1980464071,-1.9799127065 \backslash \mathrm{C},-5.54$ $6933298,3.2209301528,-1.2988263815 \backslash \mathrm{S},-7.3153360891,3.2079980973,-0.857$ $3200166 \backslash \mathrm{H},-5.0847221303,3.9328979414,-0.6096399896 \backslash \mathrm{H},-5.4311347691,3.6$ $38368325,-2.302682415 \backslash \mathrm{H},-7.7001236496,2.3499405392,-1.8150247779 \backslash \backslash$ Vers 
ion=ES64L-G09RevD. 01 \State $=1-\mathrm{A} \backslash \mathrm{HF}=-952.3085142 \backslash \mathrm{MP} 2=-953.077932 \backslash \mathrm{RMSD}=2$. $711 \mathrm{e}-09 \backslash \mathrm{RMSF}=9.835 \mathrm{e}-06 \backslash \mathrm{Dipole}=0.0983726,-0.8382783,-0.4244481 \backslash \mathrm{PG}=\mathrm{C} 02$ [ $\mathrm{X}(\mathrm{C} 4 \mathrm{H} 10 \mathrm{~S} 2) \mathrm{]} \backslash \backslash \mathrm{Q}$

Product - Product $18-)$ HSCH $2 \mathrm{C}(\mathrm{CH} 3) 2-\mathrm{C}(\mathrm{CH} 3) 2 \mathrm{CH} 2 \mathrm{SH}$

$1 \backslash 1 \backslash G I N C-R 397 \backslash F O p t \backslash R M P 2-F C \backslash 6-31 G(d) \backslash C 8 H 18 S 2 \backslash R O O T \backslash 15-J a n-2016 \backslash 0 \backslash \backslash \#$ MP2 / 6-31G(d) SCF=Tight INT (grid=ultrafine) OPT IOP $(2 / 17=4)$ maxdisk=4160749 $568 \backslash \backslash$ diMetk.MP $2 \backslash \backslash 0,1 \backslash \mathrm{S},-2.7557721725,1.992718503,0.6892756623 \backslash \mathrm{H},-2.691$ $4208632,1.4639617753,1.9193556114 \backslash \mathrm{C},-4.5723712395,2.1729932162,0.61251$ $04894 \backslash \mathrm{C},-5.3933621908,0.8890184783,0.3576053643 \backslash \mathrm{H},-4.7270181328,2.8761$ $56222,-0.2095324207 \backslash \mathrm{H},-4.8955596096,2.673045445,1.5285931356 \backslash \mathrm{C},-4.9796$ $028385,-0.1902362635,1.3669513243 \backslash C,-5.0547140572,0.3774278999,-1.0493$ $48306 \backslash \mathrm{H},-5.6357157525,-1.0636027857,1.3171208517 \backslash \mathrm{H},-3.9659362116,-0.53$ $43230784,1.1456094251 \backslash \mathrm{H},-4.9928640044,0.1826280672,2.3958138081 \backslash \mathrm{H},-5.4$ $639084859,-0.6206201519,-1.2261841693 \backslash \mathrm{H},-5.4268501665,1.0460476769,-1$. $8320683956 \backslash \mathrm{H},-3.9683820614,0.3097363091,-1.1558937845 \backslash \mathrm{C},-6.9297108055$, $1.23992902,0.4987435595 \backslash \mathrm{C},-7.7538018672,0.0892064567,-0.1211649248 \backslash \mathrm{C},-$ $7.3224062942,1.4128729362,1.9720563846 \backslash \mathrm{C},-7.2865939065,2.535731931,-0$. $2425132697 \backslash \mathrm{S},-9.5680312615,0.167778007,0.0808535099 \backslash \mathrm{H},-7.6153052766,0$. $0817610222,-1.2050792265 \backslash \mathrm{H},-7.4196977965,-0.881617185,0.2524492847 \backslash \mathrm{H},-$ $8.3740146758,2.6501943272,-0.2641107582 \backslash \mathrm{H},-6.8746529929,3.4152291588,0$ $.2590838009 \backslash \mathrm{H},-6.9298852707,2.5306261667,-1.2773669438 \backslash \mathrm{H},-8.3373999529$ $, 1.813034721,2.0396458762 \backslash \mathrm{H},-7.2957278025,0.4649664526,2.5184288264 \backslash \mathrm{H}$, $-6.6623820492,2.1165715694,2.4867540163 \backslash \mathrm{H},-9.6110472613,-0.2200168973$, $1.3632672685 \backslash \backslash$ Version=ES64L-G09RevD.01 \State $=1-A \backslash H F=-1108.4237276 \backslash M P 2=$ $-1109.742507 \backslash \mathrm{RMSD}=3.804 \mathrm{e}-09 \backslash \mathrm{RMSF}=2.634 \mathrm{e}-06 \backslash \mathrm{Dipole}=0.0049704,-0.2126638$ , $0.5829552 \backslash \mathrm{PG}=\mathrm{C} 01 \quad[\mathrm{X}(\mathrm{C} 8 \mathrm{H} 18 \mathrm{~S} 2)] \backslash \backslash @$

Product - Product 19-) $\mathrm{CH} 3 \mathrm{SCH} 2 \mathrm{CH} 2-\mathrm{CH} 2 \mathrm{CH} 2 \mathrm{SCH} 3$

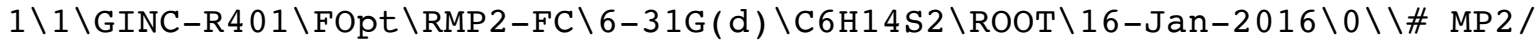
6-31G(d) SCF=Tight INT(grid=ultrafine) OPT IOP(2/17=4) maxdisk=4160749 $568 \backslash \backslash$ diMetk.MP $2 \backslash \backslash 0,1 \backslash S,-2.7330859917,2.9524558707,0.4763604308 \backslash \mathrm{C},-2.93$ $43871696,2.9823796919,2.2741888908 \backslash \mathrm{C},-4.4692140003,2.7457011815,-0.020$ $5986377 \backslash \mathrm{C},-5.1003563659,1.420804924,0.4030159988 \backslash \mathrm{H},-4.4700878558,2.836$ $7777003,-1.1126613461 \backslash \mathrm{H},-5.0460967174,3.5895927731,0.374607659 \backslash \mathrm{H},-6.17$ $21703188,1.4568612664,0.1657064201 \backslash \mathrm{H},-5.0280991399,1.3108776005,1.4893$ $657852 \backslash \mathrm{H},-1.9642762513,3.2456303589,2.7011063895 \backslash \mathrm{H},-3.6605166629,3.743$ $5589615,2.5716462398 \backslash \mathrm{H},-3.237604254,2.0110162025,2.6699695784 \backslash \mathrm{C},-4.470$ $9144892,0.2072660937,-0.2737814349 \backslash \mathrm{C},-4.8124354044,-1.1138991975,0.412$ $5279266 \backslash \mathrm{H},-4.8107707269,0.1530610783,-1.3168353632 \backslash \mathrm{H},-3.3843437314,0.3$ $320845024,-0.3090889608 \backslash \mathrm{S},-4.1461460825,-1.2897491711,2.0944858372 \backslash \mathrm{H},-$ $5.898014925,-1.2189392677,0.5192074756 \backslash \mathrm{H},-4.4633289296,-1.9607258942,-$ $0.1892558808 \backslash \mathrm{C},-2.3761639083,-1.299142171,1.7194120761 \backslash \mathrm{H},-1.8532049384$ $,-1.5428132747,2.6466733185 \backslash \mathrm{H},-2.1398178017,-2.0667606864,0.9777652545$ $\backslash \mathrm{H},-2.025736335,-0.3271745433,1.3664103429 \backslash \backslash$ Version=ES64L-G09RevD.01 $\backslash \mathrm{S}$ tate $=1-\mathrm{A} \backslash \mathrm{HF}=-1030.376204 \backslash \mathrm{MP} 2=-1031.4151442 \backslash \mathrm{RMSD}=8.200 \mathrm{e}-09 \backslash \mathrm{RMSF}=8.971 \mathrm{e}-$ $06 \backslash$ Dipole $=-0.2320434,-0.0028509,-0.2104493 \backslash \mathrm{PG}=\mathrm{C} 01 \quad[\mathrm{X}(\mathrm{C} 6 \mathrm{H} 14 \mathrm{~S} 2)] \backslash \backslash @$

Product - Product 20-) $\mathrm{CH} 3 \mathrm{SCH} 2 \mathrm{C}(\mathrm{CH} 3) 2-\mathrm{C}(\mathrm{CH} 3) 2 \mathrm{CH} 2 \mathrm{SCH} 3$

$1 \backslash 1 \backslash \mathrm{GINC}-\mathrm{R} 3481 \backslash \mathrm{FOpt} \backslash \mathrm{RMP} 2-\mathrm{FC} \backslash 6-31 \mathrm{G}(\mathrm{d}) \backslash \mathrm{C} 10 \mathrm{H} 22 \mathrm{~S} 2 \backslash \mathrm{ROOT} \backslash 15-\mathrm{Jan}-2016 \backslash 0 \backslash \backslash \#$ MP 2/6-31G(d) SCF=Tight INT(grid=ultrafine) OPT IOP $(2 / 17=4)$ maxdisk=68451 $04128 \backslash \backslash$ diMetk.MP2 $\backslash \backslash 0,1 \backslash S,-2.7290701542,2.1419446302,0.2722978354 \backslash \mathrm{C},-2$. $4862490873,2.5272642958,2.0236569922 \backslash C,-4.5253294602,2.4050860692,0.09$ $18559503 \backslash \mathrm{C},-5.4398487806,1.1673374822,0.235275173 \backslash \mathrm{H},-4.6414880565,2.82$ $07310245,-0.9124496578 \backslash \mathrm{H},-4.8053803382,3.1915912149,0.8003874113 \backslash \mathrm{C},-5$. $1198055284,0.4358983201,1.5448339615 \backslash \mathrm{C},-5.1165423622,0.2123201499,-0.9$ $2311463 \backslash \mathrm{H},-1.4078478775,2.5885383541,2.1870602056 \backslash \mathrm{H},-2.9263067089,3.49$ $63520857,2.2721546893 \backslash \mathrm{H},-2.8945230556,1.7592843741,2.6814136278 \backslash \mathrm{H},-5.6$ $033085228,-0.7582261388,-0.7967034435 \backslash \mathrm{H},-5.4138958959,0.623457274,-1.8$ $930887201 \backslash \mathrm{H},-4.0371940691,0.0349354032,-0.9508014694 \backslash \mathrm{H},-5.8085886597$,- 
$0.3959779598,1.7204541304 \backslash \mathrm{H},-4.1100352858,0.0189401218,1.4912350777 \backslash \mathrm{H}$, $-5.167167637,1.1021319524,2.4100221133 \backslash \mathrm{C},-6.9488595817,1.6419008648,0$. $2014701193 \backslash \mathrm{C},-7.8519270367,0.4125760342,-0.0478615891 \backslash \mathrm{C},-7.3455186947$, $2.3169504767,1.5203124563 \backslash \mathrm{C},-7.2053710327,2.6460815542,-0.9317883095 \backslash \mathrm{S}$ $,-9.6559747765,0.6699984835,0.0387162604 \backslash \mathrm{H},-7.6769329487,0.0401763471$, $-1.0605704454 \backslash \mathrm{H},-7.612653997,-0.4039008635,0.6414492089 \backslash \mathrm{C},-9.999943293$ $2,0.2103612252,1.7548488596 \backslash \mathrm{H},-11.0859554609,0.1434884833,1.8523674903$ $\backslash \mathrm{H},-9.5738963274,-0.7690220914,1.9868516342 \backslash \mathrm{H},-9.6316515444,0.94897181$ $54,2.4675480705 \backslash \mathrm{H},-8.2814991284,2.8258236784,-1.0146500478 \backslash \mathrm{H},-6.728017$ $6972,3.6097167889,-0.7357732919 \backslash \mathrm{H},-6.8515238707,2.2765218127,-1.899528$ $1293 \backslash \mathrm{H},-8.3509775186,2.7370786596,1.4259324193 \backslash \mathrm{H},-7.3477720767,1.61419$ $66859,2.3574461388 \backslash \mathrm{H},-6.6691715347,3.1396793916,1.771219908 \backslash \backslash$ Version= $\mathrm{E}$ S64L-G09RevD.01 \State $=1-A \backslash H F=-1186.4875839 \backslash M P 2=-1188.0768003 \backslash \mathrm{RMSD}=3.05$ $2 e-09 \backslash \mathrm{RMSF}=1.893 e-06 \backslash \mathrm{Dipole}=-0.026877,-0.0197517,0.9213722 \backslash \mathrm{PG}=\mathrm{C} 01][\mathrm{X}(\mathrm{C}$ $10 \mathrm{H} 22 \mathrm{~S} 2)] \backslash \backslash @$

Product - Product 21-) $\mathrm{CH} 3 \mathrm{SCH} 2-\mathrm{CH} 2 \mathrm{SCH} 3$

$1 \backslash 1 \backslash G I N C-R 360 \backslash F O p t \backslash R M P 2-F C \backslash 6-31 G(d) \backslash C 4 H 10 S 2 \backslash R O O T \backslash 15-J a n-2016 \backslash 0 \backslash \backslash \#$ MP $2 /$ 6-31G(d) SCF=Tight INT(grid=ultrafine) OPT IOP(2/17=4) maxdisk=9395240 $96 \backslash \backslash$ diMetk.MP2 $\backslash \backslash 0,1 \backslash \mathrm{H},-2.6876337927,2.188751745,-0.1607181021 \backslash \mathrm{C},-2.842$ $7326105,1.7947981203,0.8467210656 \backslash \mathrm{H},-2.7551448846,0.7070143836,0.83290$ $88004 \backslash \mathrm{H},-2.0620988043,2.1931286901,1.497901963 \backslash \mathrm{S},-4.4281288633,2.34961$ $33686,1.5184730459 \backslash \mathrm{C},-5.540706613,1.7678368226,0.2078207328 \backslash \mathrm{H},-5.19244$ $94946,2.1380756483,-0.7618035509 \backslash \mathrm{C},-5.6982462961,0.2527033289,0.170775$ $4794 \backslash \mathrm{S},-6.8108240457,-0.3290732171,-1.1398768338 \backslash \mathrm{H},-4.7370861154,-0.22$ $69317959,-0.0349665045 \backslash \mathrm{H},-6.0465034145,-0.1175354968,1.140399763 \backslash \mathrm{H},-6$. $5018667936,2.2474719474,0.4135627166 \backslash \mathrm{C},-8.3962202986,0.2257420312,-0.4$ $681248535 \backslash \mathrm{H},-9.1768541048,-0.1725885386,-1.1193057508 \backslash \mathrm{H},-8.5513191164$, $-0.1682115935,0.5393143143 \backslash \mathrm{H},-8.4838080245,1.3135257679,-0.4543125883 \backslash$ $\backslash$ Version=ES64L-G09RevD.01 \State $=1-A G \backslash H F=-952.3099736 \backslash M P 2=-953.0824734 \backslash$ $\mathrm{RMSD}=6.659 \mathrm{e}-09 \backslash \mathrm{RMSF}=4.313 \mathrm{e}-05 \backslash \mathrm{Dipole}=0 ., 0 ., 0 . \backslash \mathrm{PG}=\mathrm{CI}[\mathrm{X}(\mathrm{C} 4 \mathrm{H} 10 \mathrm{~S} 2)] \backslash \backslash @$

Product - Product 22-) $\mathrm{CH} 3 \mathrm{SC}(\mathrm{CH} 3) 2-\mathrm{C}(\mathrm{CH} 3) 2 \mathrm{SCH} 3$

$1 \backslash 1 \backslash G I N C-R 417 \backslash F O p t \backslash R M P 2-F C \backslash 6-31 G(d) \backslash C 8 H 18 S 2 \backslash R O O T \backslash 15-J a n-2016 \backslash 0 \backslash \backslash \#$ MP $/$ 6-31G(d) SCF=Tight INT(grid=ultrafine) OPT IOP(2/17=4) maxdisk=2281701 $376 \backslash \backslash$ diMetk.MP $2 \backslash \backslash 0,1 \backslash \mathrm{H},-2.8030790183,3.0306544395,0.2873550476 \backslash \mathrm{C},-2.85$ $33494202,2.2409188373,1.0380315809 \backslash \mathrm{H},-2.4356004251,1.3187565195,0.6330$ $76918 \backslash \mathrm{H},-2.2454638006,2.5469070904,1.8933090464 \backslash \mathrm{S},-4.5284872603,2.0332$ $927179,1.693033261 \backslash \mathrm{C},-5.5804680264,1.5933067677,0.2298684429 \backslash \mathrm{C},-5.0849$ $504061,2.3320707778,-1.0125277013 \backslash \mathrm{C},-5.6479847984,0.0401599386,0.03286$ $34546 \backslash S,-6.6962988342,-0.3996450065,-1.4329850186 \backslash \mathrm{C},-4.269393843,-0.60$ $60902098,-0.0963877629 \backslash C,-6.3756224868,-0.6362487045,1.2030703378 \backslash C,-6$ $.9560613351,2.165891105,0.5990020683 \backslash \mathrm{C},-5.5565714802,-0.4683175706,-2$. $8381640916 \backslash \mathrm{H},-6.1683336835,-0.7744971881,-3.6906043118 \backslash \mathrm{H},-5.1049247193$ $, 0.4967617692,-3.0695142887 \backslash \mathrm{H},-4.7763779886,-1.2170579908,-2.695298452$ $1 \backslash \mathrm{H},-7.7028037334,1.8424558182,-0.1320250446 \backslash \mathrm{H},-7.2796813308,1.8520555$ $721,1.594508735 \backslash \mathrm{H},-6.9096518023,3.2579544339,0.5946610571 \backslash \mathrm{H},-5.8311716$ $953,2.240374519,-1.8060682526 \backslash \mathrm{H},-4.9641074829,3.3956845854,-0.78606755$ $62 \backslash \mathrm{H},-4.1306133772,1.952845246,-1.3853401193 \backslash \mathrm{H},-6.2874566169,-1.721668$ $827,1.108916524 \backslash \mathrm{H},-5.924925944,-0.3274274831,2.1507714053 \backslash \mathrm{H},-7.4403565$ $433,-0.3909481523,1.22369059 \backslash \mathrm{H},-4.3803509385,-1.6692160113,-0.33008327$ $82 \backslash \mathrm{H},-3.6523477327,-0.1492946136,-0.8736600118 \backslash \mathrm{H},-3.7441729476,-0.5253$ $034575,0.8589014784 \backslash \backslash$ Version=ES64L-G09RevD. $01 \backslash$ State $=1-A \backslash H F=-1108.42376$ $94 \backslash \mathrm{MP} 2=-1109.7498471 \backslash \mathrm{RMSD}=9.826 \mathrm{e}-09 \backslash \mathrm{RMSF}=1.702 \mathrm{e}-05 \backslash \mathrm{Dipole}=0.7836713,0$. $0386853,-0.5735637 \backslash \mathrm{PG}=\mathrm{C} 02[\mathrm{X}(\mathrm{C} 8 \mathrm{H} 18 \mathrm{~S} 2)] \backslash \backslash @$

Product - Product 23-) $\mathrm{CH} 3 \mathrm{CH} 2 \mathrm{SCH} 2-\mathrm{CH} 2 \mathrm{SCH} 2 \mathrm{CH} 3$

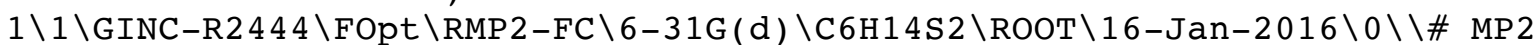
/6-31G(d) SCF=Tight INT(grid=ultrafine) OPT IOP(2/17=4) maxdisk=939524 $096 \backslash \backslash$ diMetk.MP $2 \backslash \backslash 0,1 \backslash \mathrm{H},-2.7221105981,2.2583862228,-0.1394990784 \backslash \mathrm{C},-2.8$ 
$121323205,1.8182716482,0.8585848171 \backslash \mathrm{H},-2.8305477637,0.7330120365,0.721$ $7015002 \backslash \mathrm{C},-1.630727899,2.2389222012,1.722937559 \backslash \mathrm{S},-4.4298119546,2.3984$ $647361,1.4510025339 \backslash \mathrm{C},-4.5308757072,1.5991191696,3.0788735677 \backslash \mathrm{H},-5.451$ $0115343,1.9974243013,3.5169611351 \backslash \mathrm{C},-4.5891710697,0.079443405,3.008121$ $5654 \backslash \mathrm{S},-4.6902348224,-0.7199021614,4.6359925991 \backslash \mathrm{H},-5.4141877884,-0.240$ $7648093,2.3660296728 \backslash \mathrm{H},-3.6690352426,-0.3188617266,2.5700339979 \backslash \mathrm{H},-1.6$ $120116526,3.3240713856,1.8486267992 \backslash \mathrm{H},-1.6699164203,1.7810948012,2.714$ $5438335 \backslash \mathrm{H},-0.6929938176,1.9268614813,1.2523713715 \backslash \mathrm{H},-3.7058589886,1.91$ $93273839,3.7209654603 \backslash \mathrm{C},-6.3079144564,-0.1397090735,5.2284103159 \backslash \mathrm{C},-7$. $489318878,-0.5603596266,4.364057574 \backslash \mathrm{H},-6.3979361789,-0.5798236482,6.22$ $64942114 \backslash \mathrm{H},-6.2894990133,0.9455505382,5.3652936328 \backslash \mathrm{H},-8.4270529594,-0$. $2482989066,4.8346237616 \backslash \mathrm{H},-7.4501303566,-0.1025322265,3.3724512995 \backslash \mathrm{H},-$ $7.5080351243,-1.6455088109,4.2383683339 \backslash \backslash$ Version=ES64L-G09RevD. $01 \backslash$ Stat $e=1-A G \backslash H F=-1030.3779614 \backslash M P 2=-1031.416813 \backslash R M S D=7.027 e-09 \backslash R M S F=5.963 e-06$ $\backslash \mathrm{Dipole}=0 ., 0 ., 0 . \backslash \mathrm{PG}=\mathrm{CI} \quad[\mathrm{X}(\mathrm{C} 6 \mathrm{H} 14 \mathrm{~S} 2)] \backslash \backslash @$

Product - Product 24-) $\mathrm{CH} 3 \mathrm{CH} 2 \mathrm{SC}(\mathrm{CH} 3) 2-\mathrm{C}(\mathrm{CH} 3) 2 \mathrm{SCH} 2 \mathrm{CH} 3$

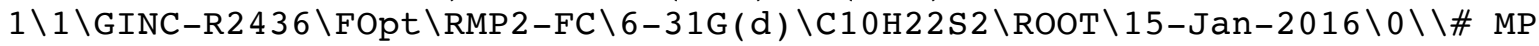
2/6-31G(d) SCF=Tight INT(grid=ultrafine) OPT IOP(2/17=4) maxdisk=68451 $04128 \backslash \backslash \mathrm{diMetk} . \mathrm{MP} 2 \backslash \backslash 0,1 \backslash \mathrm{H},-2.9225041874,3.3081352102,0.0195158114 \backslash \mathrm{C},-2$. $905912027,2.6176851988,0.8662377196 \backslash \mathrm{H},-2.5110655318,1.6603858216,0.521$ $974602 \backslash \mathrm{C},-2.0383853659,3.1891761759,1.9840183089 \backslash \mathrm{S},-4.5918343595,2.399$ $9362706,1.5161276944 \backslash \mathrm{C},-5.572201308,1.6220104847,0.1512255119 \backslash \mathrm{C},-5.078$ $2526025,2.1233151009,-1.2057227056 \backslash \mathrm{C},-5.5765934254,0.0573075826,0.2324$ $231945 \backslash \mathrm{S},-3.8774445095,-0.6647811955,0.088455809 \backslash \mathrm{C},-6.2591180549,-0.44$ $79266671,1.5034458497 \backslash \mathrm{C},-6.3037829036,-0.5535660454,-0.9785644845 \backslash \mathrm{H},-4$ $.1099931964,1.6931727818,-1.4722835736 \backslash \mathrm{H},-5.7994412796,1.8641493617,-1$ $.9865163941 \backslash \mathrm{H},-4.9879167564,3.2133797072,-1.1895124097 \backslash \mathrm{H},-2.4354235656$ $, 4.1416178255,2.3453583202 \backslash \mathrm{H},-1.9810564525,2.5007395178,2.8313736217 \backslash \mathrm{H}$ $,-1.0232031511,3.3610214316,1.6140615872 \backslash \mathrm{C},-6.9835087841,2.1883211107$, $0.3877963646 \backslash \mathrm{H},-6.9730883334,3.2651689538,0.2010811842 \backslash \mathrm{H},-7.7129974253$ $, 1.7324718658,-0.2879812196 \backslash \mathrm{H},-7.3223980406,2.0347144151,1.4154414118 \backslash$ $C,-3.2549728682,-0.8344267279,1.7901770363 \backslash \mathrm{C},-1.8233374156,-1.35800668$ $71,1.7200211563 \backslash \mathrm{H},-3.2888548347,0.1303945974,2.2989154457 \backslash \mathrm{H},-3.8807638$ $412,-1.5382630202,2.3441938589 \backslash \mathrm{H},-1.4309842597,-1.5005210608,2.7313284$ $051 \backslash \mathrm{H},-1.7781776738,-2.3177809449,1.1983459668 \backslash \mathrm{H},-1.1702940764,-0.6547$ $66959,1.1963505111 \backslash \mathrm{H},-6.4006520065,-1.6322153461,-0.8312400646 \backslash \mathrm{H},-7.30$ $75078199,-0.1338343172,-1.0925343212 \backslash \mathrm{H},-5.756026762,-0.3956068838,-1.9$ $110382915 \backslash \mathrm{H},-6.1500633865,-1.5338362803,1.5780888384 \backslash \mathrm{H},-5.8402955682,0$ $.0111203147,2.4020375154 \backslash \mathrm{H},-7.3298365288,-0.2261015291,1.4677480881 \backslash \backslash \mathrm{V}$ ersion=ES64L-G09RevD. $01 \backslash$ State $=1-A \backslash H F=-1186.4915192 \backslash M P 2=-1188.0844527 \backslash R$ $M S D=7.061 e-09 \backslash R M S F=3.656 e-06 \backslash D i p o l e=-0.059766,-0.0012454,-0.0272327 \backslash P G$ $=\mathrm{C} 02[\mathrm{X}(\mathrm{C} 10 \mathrm{H} 22 \mathrm{~S} 2)] \backslash \backslash @$

Product - Product 25-) HSSCH2-CH2SSH

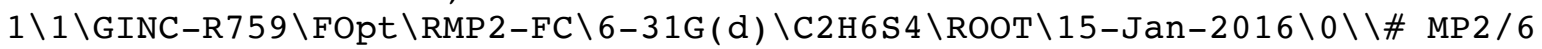
$-31 \mathrm{G}(\mathrm{d})$ SCF=Tight INT(grid=ultrafine) OPT IOP(2/17=4) maxdisk=68451041 $28 \backslash \backslash$ diMetk.MP2 $\backslash \backslash 0,1 \backslash S,-0.0507485937,0.1246891429,-0.9220502765 \backslash \mathrm{C}, 0.984$ $1003745,1.3036251386,0.0003598852 \backslash \mathrm{S},-1.9649764899,0.8643390634,-0.6970$ $273172 \backslash \mathrm{H},-2.0011566299,1.7027263918,-1.7480109696 \backslash \mathrm{H}, 0.414813483,1.6881$ $744076,0.8514143362 \backslash \mathrm{H}, 1.8085438759,0.7051021026,0.4057247311 \backslash \mathrm{C}, 1.56766$ $31655,2.436604944,-0.8311222923 \backslash \mathrm{H}, 2.2678884438,3.0162127879,-0.2205850$ $102 \backslash \mathrm{H}, 2.1174197477,2.0314098251,-1.6878597305 \backslash \mathrm{S}, 0.3558594404,3.5737008$ $499,-1.566986004 \backslash \mathrm{S},-0.4384653701,4.4894624247,0.1024490918 \backslash \mathrm{H},-1.409322$ $4471,3.6000339217,0.388060556 \backslash \backslash$ Version=ES64L-G09RevD . 01 \State=1-A $\backslash H F=-$ $1669.2601421 \backslash M P 2=-1670.0067294 \backslash \mathrm{RMSD}=4.612 \mathrm{e}-09 \backslash \mathrm{RMSF}=4.746 \mathrm{e}-06 \backslash \mathrm{Dipole}=0$. $4956557,-0.0413112,0.2893569 \backslash \mathrm{PG}=\mathrm{C} 01 \quad[\mathrm{X}(\mathrm{C} 2 \mathrm{H} 6 \mathrm{~S} 4)] \backslash \backslash \mathrm{a}$

Product - Product 26-) HSSC ( CH3) 2-C(CH3) 2SSH

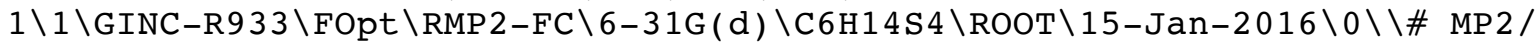


6-31G(d) SCF=Tight INT(grid=ultrafine) OPT IOP (2/17=4) maxdisk=6845104 $128 \backslash \backslash$ diMetk.MP $2 \backslash \backslash 0,1 \backslash S, 0.0056689986,0.0783150674,-0.5427791575 \backslash \mathrm{C}, 1.346$ $6167138,0.8790109906,0.4708834934 \backslash S,-1.377374033,1.5419205223,-0.99804$ $75342 \backslash \mathrm{H},-2.1802576224,1.4205240496,0.0742033239 \backslash \mathrm{C}, 0.732572502,1.839567$ $6568,1.4850973557 \backslash \mathrm{C}, 2.4129893242,1.5395986651,-0.4589660973 \backslash \mathrm{S}, 3.782401$ $7083,2.2924181887,0.5533279278 \backslash \mathrm{C}, 3.17551632,0.4912471402,-1.2819219245$ $\backslash \mathrm{C}, 1.8376578105,2.5795229331,-1.4159929138 \backslash \mathrm{H}, 1.487682997,2.1298254269$, $2.2197727522 \backslash \mathrm{H}, 0.3311161277,2.7431982136,1.024618133 \backslash \mathrm{H},-0.0766167827,1$ $.3339139213,2.0211307925 \backslash \mathrm{C}, 1.9232602102,-0.3250670406,1.2294499081 \backslash \mathrm{H}, 1$ $.1951289638,-0.6799629007,1.9631950621 \backslash \mathrm{H}, 2.1669200943,-1.161030518,0.5$ $679678812 \backslash \mathrm{H}, 2.832124523,-0.0233655142,1.758330928 \backslash \mathrm{H}, 2.6549519872,3.071$ $5445745,-1.9524160465 \backslash \mathrm{H}, 1.2034382519,2.0859315919,-2.1564809357 \backslash \mathrm{H}, 1.24$ $77083597,3.3433961,-0.9078453479 \backslash \mathrm{H}, 3.8167367108,0.9923234762,-2.011488$ $2685 \backslash \mathrm{H}, 3.8096380406,-0.1491818519,-0.6626450105 \backslash \mathrm{H}, 2.4629225353,-0.1405$ $733226,-1.8202399384 \backslash \mathrm{S}, 3.169840169,4.1853373466,1.1045480945 \backslash \mathrm{H}, 3.63327$ $98565,4.8776644674,0.0486182304 \backslash \backslash$ Version=ES64L-G0 9RevD. $01 \backslash \mathrm{HF}=-1825.379$ $504 \backslash M P 2=-1826.6782449 \backslash \mathrm{RMSD}=7.401 \mathrm{e}-09 \backslash \mathrm{RMSF}=2.488 \mathrm{e}-06 \backslash \mathrm{Dipole}=-0.1049699$, $0.1765552,0.0050472 \backslash \mathrm{PG}=\mathrm{C} 02 \quad[\mathrm{X}(\mathrm{C} 6 \mathrm{H} 14 \mathrm{~S} 4)] \backslash \backslash @$

Product - Product 27-) $\mathrm{CH} 3 \mathrm{SSCH} 2-\mathrm{CH} 2 \mathrm{SSCH} 3$

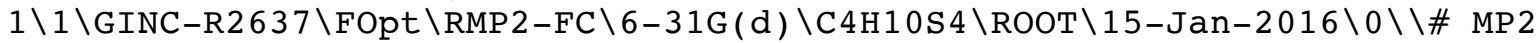
/6-31G(d) SCF=Tight INT(grid=ultrafine) OPT IOP $(2 / 17=4)$ maxdisk $=684510$ $4128 \backslash \backslash$ diMetk.MP $2 \backslash \backslash 0,1 \backslash \mathrm{S},-0.3974831284,0.6213492319,-0.3176715554 \backslash \mathrm{C}, 1.1$ $909020322,1.3488559867,0.2023454205 \backslash S,-1.5048094664,2.2977749116,-0.76$ $22970379 \backslash \mathrm{C},-0.9571014462,2.6696244798,-2.450906034 \backslash \mathrm{H}, 1.8905578297,0.50$ $9155873,0.2698888232 \backslash \mathrm{H}, 1.5505457584,2.0239785127,-0.5785962763 \backslash \mathrm{C}, 1.095$ $493225,2.073480516,1.532166982 \backslash \mathrm{H}, 0.289720246,2.8119109435,1.5108508162$ $\backslash \mathrm{H}, 0.8837073236,1.3673731569,2.3419421091 \backslash \mathrm{S}, 2.6532724476,2.897961665,1$ $.9971464229 \backslash \mathrm{S}, 2.7490961754,4.426464811,0.6228238829 \backslash \mathrm{C}, 1.6875444642,5.6$ $827202429,1.3874066272 \backslash \mathrm{H},-1.5425966061,3.5291945838,-2.7880930138 \backslash \mathrm{H}, 0$. $1016472495,2.9336826017,-2.4750680187 \backslash \mathrm{H},-1.1453465305,1.8233019918,-3$. $1124959023 \backslash \mathrm{H}, 1.7243995799,6.5615067927,0.7379943501 \backslash \mathrm{H}, 0.6538269423,5.3$ $393512725,1.456581253 \backslash \mathrm{H}, 2.0584557458,5.9502679902,2.3774784143 \backslash \backslash$ Versio $\mathrm{n}=\mathrm{ES} 64 \mathrm{~L}-\mathrm{G} 09$ RevD $.01 \backslash \mathrm{HF}=-1747.3363135 \backslash \mathrm{MP} 2=-1748.350694 \backslash \mathrm{RMSD}=9.782 \mathrm{e}-09 \backslash \mathrm{RM}$ $\mathrm{SF}=5.522 \mathrm{e}-07 \backslash \mathrm{Dipole}=-0.1889743,0.5987618,-0.3398258 \backslash \mathrm{PG}=\mathrm{C} 02 \quad[\mathrm{X}(\mathrm{C} 4 \mathrm{H} 10 \mathrm{~S} 4)$ ]$\backslash \backslash @$

Product - Product 28-) $\mathrm{CH} 3 \mathrm{SSC}(\mathrm{CH} 3) 2-\mathrm{C}(\mathrm{CH} 3) 2 \mathrm{SSCH} 3$

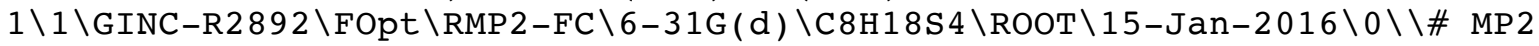
/6-31G(d) SCF=Tight INT(grid=ultrafine) OPT IOP $(2 / 17=4)$ maxdisk $=684510$ $4128 \backslash \backslash$ diMetk.MP $2 \backslash \backslash 0,1 \backslash S,-0.588197722,0.2488922353,0.0479804152 \backslash \mathrm{C}, 1.127$ $3825684,0.9017692941,0.3750405048 \backslash \mathrm{S},-1.330574837,1.2083617849,-1.61312$ $73912 \backslash \mathrm{C},-0.9102506198,0.0663535784,-2.9586481823 \backslash \mathrm{C}, 1.763906178,-0.2572$ $175541,1.1548465211 \backslash \mathrm{C}, 1.0558855515,2.2056468318,1.2346860822 \backslash \mathrm{S}, 2.77351$ $27296,2.8204066598,1.6197311703 \backslash \mathrm{C}, 0.2337745365,3.3190326605,0.59314401$ $19 \backslash \mathrm{C}, 0.4964040019,1.9299531397,2.6373562597 \backslash \mathrm{H}, 2.7385822697,0.052451922$ $9,1.5434126177 \backslash \mathrm{H}, 1.1440874126,-0.5885918584,1.9925079039 \backslash \mathrm{H}, 1.909877980$ $1,-1.1113856186,0.4886029743 \backslash \mathrm{C}, 1.8804154983,1.0747585231,-0.9402871675$ $\backslash \mathrm{H}, 1.8395819646,0.1385827933,-1.50425337 \backslash \mathrm{H}, 1.4683601853,1.8740786733,-$ $1.5591617231 \backslash \mathrm{H}, 2.9314823837,1.2963466898,-0.7400201502 \backslash \mathrm{H}, 0.3455268266$, $2.8754692751,3.1646385642 \backslash \mathrm{H},-0.4674652284,1.4190378263,2.554930962 \backslash \mathrm{H}, 1$ $.167133334,1.3132686903,3.2419083886 \backslash \mathrm{H}, 0.2770708266,4.209346837,1.2268$ $760929 \backslash \mathrm{H}, 0.5887501491,3.5826531359,-0.4049564632 \backslash \mathrm{H},-0.8127753832,3.013$ $3293655,0.520835282 \backslash \mathrm{S}, 3.401715379,3.987098898,0.0464621551 \backslash \mathrm{C}, 2.9528248$ $912,5.6610694176,0.5827469862 \backslash \mathrm{H},-1.4373457566,0.4301859623,-3.84522340$ $18 \backslash \mathrm{H}, 0.1611302736,0.0577446375,-3.1594512495 \backslash \mathrm{H},-1.254967793,-0.9426068$ $408,-2.7287764323 \backslash \mathrm{H}, 3.4235551254,6.3468666636,-0.1274037658 \backslash \mathrm{H}, 1.873695$ $0357,5.8139798637,0.5640345648 \backslash \mathrm{H}, 3.3409334835,5.8619929743,1.582083527$ $8 \backslash \backslash$ Version $=E S 64 L-G 09$ RevD. $01 \backslash \mathrm{HF}=-1903.4503619 \backslash \mathrm{MP} 2=-1905.0208252 \backslash \mathrm{RMSD}=6$. $923 e-09 \backslash \mathrm{RMSF}=3.303 e-06 \backslash \mathrm{Dipole}=-0.0129929,0.2419526,-0.368065 \backslash \mathrm{PG}=\mathrm{C} 02 \quad[\mathrm{X}$ 
$(\mathrm{C} 8 \mathrm{H} 18 \mathrm{~S} 4)] \backslash \backslash @$ 